\title{
Metal-Free Transfer Hydrobromination of C-C Triple Bonds
}

\author{
Weiqiang Chen and Martin Oestreich* \\ Institut für Chemie, Technische Universität Berlin, \\ Straße des 17. Juni 115, 10623 Berlin, Germany \\ martin.oestreich@tu-berlin.de
}

\section{Supporting Information}

Table of Contents

1 General Information

2 Synthesis of Parent Surrogates 1-3

2.1 Synthesis of 3-(2-bromoethyl)-3-methylcyclohexa-1,4-diene (1) ................................................. 3

2.2 Synthesis of 3-(2-bromoethyl)-1,3,5-trimethylcyclohexa-1,4-diene (2) …................................. 110

2.3 Synthesis of 1-(2-bromoethyl)-1,4-dihydro-1,1'-biphenyl (3) .................................................. S16

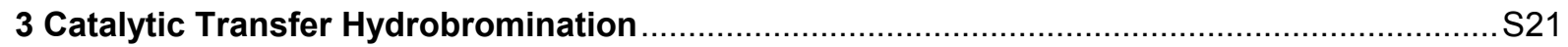

3.1 General procedures for catalytic transfer hydrobromination ................................................ S21

3.2 Substrates scope with unactivated alkynes...................................................................... 22

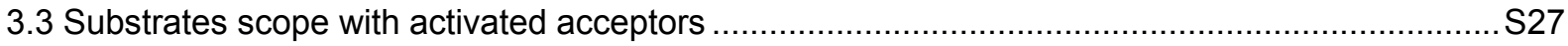

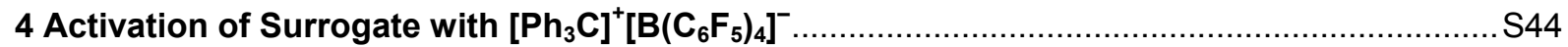

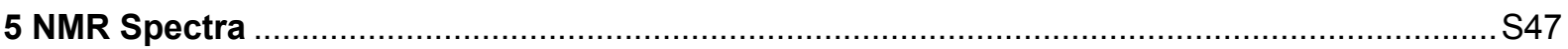

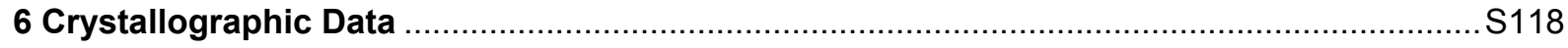

6.1 Molecular Structure of 1-(2-bromodoethyl)-1,4-dihydro-1,1'-biphenyl (3) ................................S119

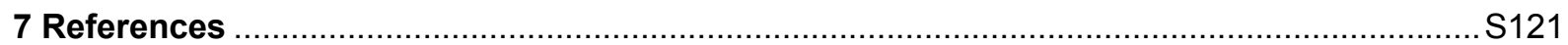




\section{General Information}

Reactions were performed in flame-dried glassware using an MBraun glove box or conventional Schlenk techniques under a static pressure of nitrogen (glove box) unless otherwise stated. Liquids and solutions were transferred with either syringes or glass pipettes. Solvents for reactions $\left(\mathrm{C}_{6} \mathrm{H}_{5} \mathrm{Cl}, \mathrm{C}_{6} \mathrm{D}_{5} \mathrm{Cl}\right.$, benzene, $\mathrm{C}_{6} \mathrm{D}_{6}$, toluene) were dried and purified following standard procedures. Technical grade solvents for extraction or chromatography (tert-butyl methyl ether, cyclohexane, diethyl ether $\left(\mathrm{Et}_{2} \mathrm{O}\right)$, and $n$-pentane) were distilled prior to use. Anhydrous 4-toluenesulfonic acid was prepared from 4-toluenesulfonic acid monohydrate via azeotropic drying with toluene. 4-Toluenesulfonic acid monohydrate was purchased from AlfaAesar. $\mathrm{Tf}_{2} \mathrm{NH}$ was purchased from Sigma Aldrich and used directly. Commercial liquid substrates were distilled, degassed with three freeze-pump-thaw cycles, and stored in a glove box over thermally activated $4-\AA \AA$ molecular sieves. Commercial solid substrates were dried under high vacuum prior to being stored in the glove box. Substrates not commercially available were prepared according to the reported procedures. Analytical thin layer chromatography (TLC) was performed on silica gel 60 F254 glass plates by Merck. Flash column chromatography was performed on silica gel 60 (40-63 $\mu \mathrm{m}, 230-400$ mesh, ASTM) by Grace using the indicated solvents. The silica was premixed with the indicated solvents prior to loading. ${ }^{1} \mathrm{H}$ and ${ }^{13} \mathrm{C} N M R$ spectra were recorded on Bruker AV400, AV500, and AV700 instruments. Chemical shifts are reported in parts per million (ppm) and are referenced to the residual solvent signals as the internal standard $\left(\mathrm{CDHCl}_{2}: \delta=5.32 \mathrm{ppm}, \mathrm{C}_{6} \mathrm{D}_{5} \mathrm{H}: \delta=7.16 \mathrm{ppm}\right.$ for ${ }^{1} \mathrm{H}$ NMR and $\mathrm{CD}_{2} \mathrm{Cl}_{2} \delta=53.84$ ppm, $\mathrm{C}_{6} \mathrm{D}_{6} \delta=128.06$ ppm for ${ }^{13} \mathrm{C}$ NMR). Chemical shifts are reported to $0.01 \mathrm{ppm}$ for ${ }^{1} \mathrm{H}$ NMR to $0.1 \mathrm{ppm}$ for ${ }^{13} \mathrm{C}$ NMR and ${ }^{19} \mathrm{~F}$ NMR spectra. Peaks that are within $0.01 \mathrm{ppm}$ for ${ }^{1} \mathrm{H}$ NMR or $0.1 \mathrm{ppm}$ for ${ }^{13} \mathrm{C}$ NMR but are still distinguishable are reported to $0.001 \mathrm{ppm}$ and $0.01 \mathrm{ppm}$, respectively. Data are reported as follows: chemical shift, multiplicity (br $s=$ broad singlet, $s=$ singlet, $d=$ doublet, $\mathrm{dd}=$ doublet of doublets, $\mathrm{dt}=$ doublet of triplets, $\mathrm{t}=$ triplet, $\mathrm{tt}=$ triplet of triplets, $q=$ quartet, $m=$ multiplet, or combinations thereof), coupling constants $(\mathrm{Hz})$, and integration. Infrared (IR) spectra were recorded on an Agilent Technologies Cary 630 FT-IR spectrometer equipped with an ATR unit or a Jasco FT/IR-4100 spectrometer, and the bands are reported in wavenumbers $\left(\mathrm{cm}^{-1}\right)$. High resolution mass spectrometry (HRMS) analysis was performed by the Analytical Facility at the Institut für Chemie, Technische Universität Berlin. 


\section{Synthesis of the Parent Surrogates 1-3}

2.1 Synthesis of the 3-(2-bromoethyl)-3-methylcyclohexa-1,4-diene (1)

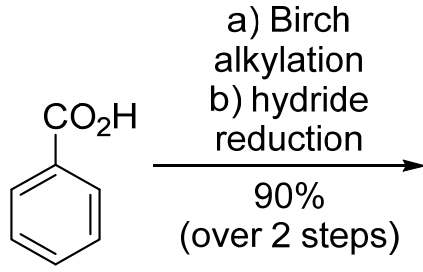

S1<smiles>CC1(CO)C=CCC=C1</smiles>

S2

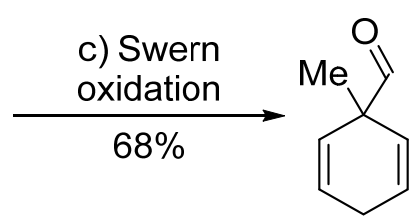

S3
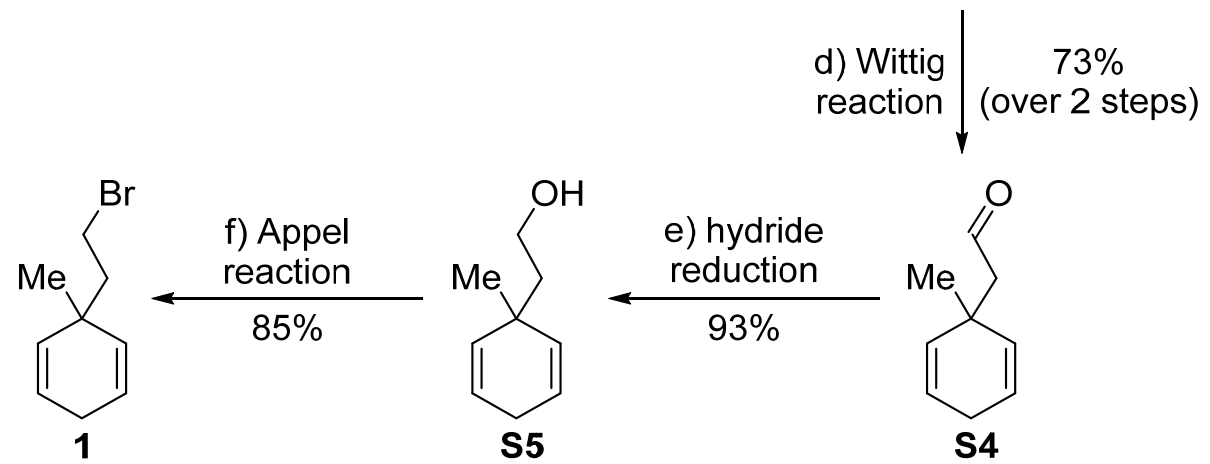<smiles>CCC1(C)C=CC=CC1(C)CC=O</smiles>

S4

Scheme S1. Synthesis of $\mathrm{HBr}$ surrogate 1. Reaction conditions: a) Na (4.0 equiv), $t$-BuOH (1.0 equiv), $\mathrm{NH}_{3},-78{ }^{\circ} \mathrm{C}, 1 \mathrm{~h}$; Mel (4.0 equiv), THF, $-78{ }^{\circ} \mathrm{C}$ to $\mathrm{RT}$, overnight; b) $\mathrm{LiAlH}_{4}\left(2.0\right.$ equiv), THF, $0{ }^{\circ} \mathrm{C}$; c) $(\mathrm{COCl})_{2}$ (1.5 equiv), DMSO (2.0 equiv), $\mathrm{CH}_{2} \mathrm{Cl}_{2}$, $-78{ }^{\circ} \mathrm{C}, 30 \mathrm{~min} ; \mathrm{Et}_{3} \mathrm{~N}$ (excess), $-78{ }^{\circ} \mathrm{C}$ to $\mathrm{RT}, 30 \mathrm{~min}$; d) $\left[\mathrm{Ph}_{3} \mathrm{PCH}_{2} \mathrm{OMe}\right]^{+} \mathrm{Cl}^{-}(1.2$ equiv), $n$-BuLi (2.5 $\mathrm{M}$ in hexanes, 1.1 equiv), $0{ }^{\circ} \mathrm{C}$ to $\mathrm{RT}$, overnight; $\mathrm{HCl}$ in $\mathrm{H}_{2} \mathrm{O}$ (32\%), $0{ }^{\circ} \mathrm{C}, 2 \mathrm{~h}$; e) $\mathrm{NaBH}_{4}$ (2.0 equiv), THF, $0{ }^{\circ} \mathrm{C}$, overnight; f) $\mathrm{Ph}_{3} \mathrm{P}$ (1.1 equiv), $\mathrm{CBr}_{4}$ (1.1 equiv), $\mathrm{CH}_{2} \mathrm{Cl}_{2}, 0^{\circ} \mathrm{C}$ to $\mathrm{RT}$, overnight. 


\subsection{1 (1-Methylcyclohexa-2,5-dien-1-yl)methanol (S2)}<smiles>CC1(CO)C=CCC=C1</smiles>

S2

$\mathrm{C}_{8} \mathrm{H}_{12} \mathrm{O}$

$124.09 \mathrm{~g} / \mathrm{mol}$

A three-necked round-bottomed flask equipped with a dry ice condenser and an overhead stirrer was purged with $\mathrm{N}_{2}$ for 15 min before being placed in a $-78{ }^{\circ} \mathrm{C}$ dry ice/acetone bath. Ammonia (approx. $200 \mathrm{~mL}$ ) was condensed, and a solution of benzoic acid (S1, $7.50 \mathrm{~g}, 61.4 \mathrm{mmol})$ and $t$-BuOH $(6.00 \mathrm{~mL}, 4.70 \mathrm{~g}, 62.0 \mathrm{mmol}$, 1.0 equiv) in THF (40 mL) was then added dropwise. Sodium $(5.60 \mathrm{~g}, 246 \mathrm{mmol}, 4.0$ equiv) was added portionwise over a period of $30 \mathrm{~min}$ to give a dark green suspension. The dark green suspension was stirred at $-78{ }^{\circ} \mathrm{C}$ for a further $60 \mathrm{~min}$. A solution of methyl iodide (15.0 mL, $34.2 \mathrm{~g}, 246 \mathrm{mmol}, 4.0$ equiv) in THF (20 mL) was added dropwise over a period of $60 \mathrm{~min}$. The resulting pale yellow suspension was stirred overnight to facilitate the evaporation of ammonia. Water $(50 \mathrm{~mL})$ was added, and the aqueous phase was acidified with $\mathrm{HCl}(32 \%$, aq.) until $\mathrm{pH}=3$ was reached, before being extracted with tert-butyl methyl ether $(3 \times 50 \mathrm{~mL})$. The combined organic phases were washed with brine $(50 \mathrm{~mL})$, dried over $\mathrm{MgSO}_{4}$ and concentrated under reduced pressure to afford 1-methylcyclohexa-2,5-diene-1-carboxylic acid as a light yellow oil.

Without further purification, a solution of crude 1-methylcyclohexa-2,5-diene-1-carboxylic acid in THF (100 mL) was added dropwise to a suspension of $\mathrm{LiAlH}_{4}(4.60 \mathrm{~g}$, $123 \mathrm{mmol}, 2.0$ equiv) in THF $(100 \mathrm{~mL})$ at $0^{\circ} \mathrm{C}$. The resulting mixture was stirred for 4 hours at $0{ }^{\circ} \mathrm{C}$ before being carefully quenched with $\mathrm{MeOH}$. $\mathrm{HCl}(1 \mathrm{M}$, aq.) was then added until the suspension disappeared. The aqueous phase was extracted with tertbutyl methyl ether $(3 \times 100 \mathrm{~mL})$, and the combined organic phases were washed with brine $(100 \mathrm{~mL})$ and concentrated under reduced pressure. The residue was purified by flash column chromatography on silica gel using cyclohexane:tert-butyl methyl ether $=20: 1$ as eluent to afford methyl 1-methylcyclohexa-2,5-dien-1-yl)methanol (S2, $6.90 \mathrm{~g}, 90 \%)$ as a yellow oil.

Data were consistent with that previously reported. ${ }^{\mathrm{S} 1}$ 
$\mathbf{R}_{\mathbf{f}}=0.52$ (cyclohexane:tert-butyl methyl ether $=2: 1$ ). $\mathbf{I R}($ ATR): $\tilde{v}=708,736,905$, 1033, 1200, 1369, 1449, 2862, 2923, 3013, $3366 \mathrm{~cm}^{-1}$. HRMS (EI) for $\mathrm{C}_{8} \mathrm{H}_{12} \mathrm{O}^{+}\left[\mathrm{M}^{+}\right]$: calculated 124.1830, found 124.1741. ${ }^{1} \mathrm{H}$ NMR $\left(500 \mathrm{MHz}, \mathrm{CD}_{2} \mathrm{Cl}_{2}\right): \delta=0.98(\mathrm{~s}, 3 \mathrm{H})$, 1.43 (br s, 1H), 2.62-2.65 (m, 2H), $3.28(\mathrm{~s}, 2 \mathrm{H}), 5.45$ (dt, $J=10.6,2.1 \mathrm{~Hz}, 2 \mathrm{H}), 5.88$ (dt, $J=10.6,3.4 \mathrm{~Hz}, 2 \mathrm{H}) \mathrm{ppm} .{ }^{13} \mathrm{C}$ NMR $\left(125 \mathrm{MHz}, \mathrm{CDCl}_{3}\right): \delta=24.8,26.4,39.1$, $70.9,126.1,131.2 \mathrm{ppm}$. 


\subsubsection{1-Methylcyclohexa-2,5-diene-1-carbaldehyde (S3)}<smiles>CC1(C=O)C=CCC=C1</smiles>

S3

$\mathrm{C}_{8} \mathrm{H}_{10} \mathrm{O}$

$122.17 \mathrm{~g} / \mathrm{mol}$

To a solution of oxalyl chloride ( $2 \mathrm{M}$ in $\mathrm{CH}_{2} \mathrm{Cl}_{2}, 14.0 \mathrm{~mL}, 28.0 \mathrm{mmol}, 1.5$ equiv) at $-78{ }^{\circ} \mathrm{C}$ was added DMSO (2.60 mL, $2.86 \mathrm{~g}, 36.5 \mathrm{mmol}, 2.0$ equiv) dropwise. The mixture was stirred for $10 \mathrm{~min}$ at $-78{ }^{\circ} \mathrm{C}$ and a solution of (1-Methylcyclohexa-2,5dien-1-yl)methanol (S2, $2.26 \mathrm{~g}, 18.2 \mathrm{mmol})$ in $\mathrm{CH}_{2} \mathrm{Cl}_{2}(20 \mathrm{~mL})$ was then added dropwise over $10 \mathrm{~min}$. The reaction was stirred for $20 \mathrm{~min}$ at $-78{ }^{\circ} \mathrm{C}$ and $\mathrm{Et}_{3} \mathrm{~N}(10$ $\mathrm{mL}$ ) was then added dropwise. The reaction was slowly warmed to RT over $30 \mathrm{~min}$. The reaction was diluted with $\mathrm{NH}_{4} \mathrm{Cl}$ (sat., aq., $100 \mathrm{~mL}$ ). The aqueous phase was extracted with $\mathrm{Et}_{2} \mathrm{O}(3 \times 100 \mathrm{~mL})$, and the combined organic phases were washed with brine $(100 \mathrm{~mL})$, dried over $\mathrm{MgSO}_{4}$ and concentrated under reduced pressure. The residue was purified by flash column chromatography on silica gel using $n$ pentane as eluent to afford 1-methylcyclohexa-2,5-diene-1-carbaldehyde (S3, $1.50 \mathrm{~g}$, $68 \%$ ) as a colorless oil.

$\mathbf{R}_{\mathbf{f}}=0.33$ (n-pentane: $\left.\mathrm{Et}_{2} \mathrm{O}=20: 1\right) . \mathbf{I R}(\mathrm{ATR}): \tilde{v}=697,756,928,1204,1371,1459$, 1695, 2816, 2927, $3017 \mathrm{~cm}^{-1}$. HRMS (APCl) for $\mathrm{C}_{8} \mathrm{H}_{11} \mathrm{O}^{+}\left[\mathrm{M}+\mathrm{H}^{+}\right]$: calculated 123.0804, found 123.0805. ${ }^{1} \mathrm{H}$ NMR (400 MHz, $\mathrm{CD}_{2} \mathrm{Cl}_{2}$ ): $\delta=1.20(\mathrm{~s}, 3 \mathrm{H}), 2.72-2.74$ $(\mathrm{m}, 2 \mathrm{H}), 5.46(\mathrm{dt}, J=10.3,2.2 \mathrm{~Hz}, 2 \mathrm{H}), 5.96-6.02(\mathrm{~m}, 2 \mathrm{H}), 9.31(\mathrm{~s}, 1 \mathrm{H}) \mathrm{ppm} .{ }^{13} \mathrm{C}$ NMR $\left(100 \mathrm{MHz}, \mathrm{CD}_{2} \mathrm{Cl}_{2}\right): \delta=21.6,26.5,50.4,126.1,127.5,199.5$ ppm. 


\subsubsection{2-(1-Methylcyclohexa-2,5-dien-1-yl)acetaldehyde (S4)}

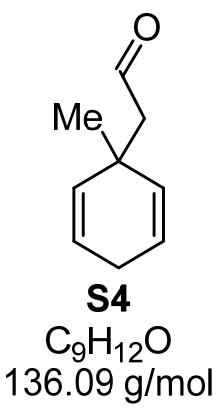

A suspension of $\left[\mathrm{Ph}_{3} \mathrm{PCH}_{2} \mathrm{OMe}\right] \mathrm{Cl}(3.30 \mathrm{~g}, 9.60 \mathrm{mmol}, 1.2$ equiv) in THF $(20 \mathrm{~mL})$ was cooled to $0^{\circ} \mathrm{C}$. $n$-BuLi (2.5 $\mathrm{M}$ in hexanes, $3.60 \mathrm{~mL}, 9.00 \mathrm{mmol}, 1.1$ equiv) was added dropwise over a period of $10 \mathrm{~min}$. After a further $60 \mathrm{~min}$ at $0{ }^{\circ} \mathrm{C}$, a solution of the 1-methylcyclohexa-2,5-diene-1-carbaldehyde (S3, $1.00 \mathrm{~g}, 8.10 \mathrm{mmol}, 1.0$ equiv) in THF (15 mL) was added, and the reaction mixture was stirred overnight, warming slowly to RT.

The reaction was cooled to $0{ }^{\circ} \mathrm{C}$ and $\mathrm{HCl}(32 \%$, aq., $20 \mathrm{~mL})$ was added dropwise. The reaction was stirred for 2 hours before being diluted with tert-butyl methyl ether $(50 \mathrm{~mL})$, and neutralized by portionwise addition of $\mathrm{K}_{2} \mathrm{CO}_{3}$ powder. The mixture was diluted with brine $(100 \mathrm{~mL})$ and extracted with tert-butyl methyl ether $(3 \times 100 \mathrm{~mL})$. The combined organic phases were dried over $\mathrm{MgSO}_{4}$ and concentrated under reduced pressure. The residue was purified by flash column chromatography on silica gel using $n$-pentane as eluent to afford 2-(1-methylcyclohexa-2,5-dien-1$\mathrm{yl}$ )acetaldehyde (S4, $0.80 \mathrm{~g}, 73 \%)$ as a light yellow oil.

$\mathbf{R}_{\mathbf{f}}=0.40$ (n-pentane:Et $\left.{ }_{2} \mathrm{O}=20: 1\right)$. IR $($ ATR): $\tilde{v}=718,943,1096,1420,1719,2959$, $3015,3423 \mathrm{~cm}^{-1}$. HRMS (EI) for $\mathrm{C}_{9} \mathrm{H}_{12} \mathrm{O}^{+}\left[\mathrm{M}^{+}\right]$: calculated 136.1940, found 136.1920. ${ }^{1} \mathrm{H}$ NMR $\left(500 \mathrm{MHz}, \mathrm{CD}_{2} \mathrm{Cl}_{2}\right): \delta=1.06(\mathrm{~s}, 3 \mathrm{H}), 2.20(\mathrm{~d}, \mathrm{~J}=3.1 \mathrm{~Hz}, 2 \mathrm{H}), 2.52-2.55(\mathrm{~m}$, 2H), 5.50 (dt, $J=10.2,2.1 \mathrm{~Hz}, 2 \mathrm{H}), 5.68-5.72(\mathrm{~m}, 2 \mathrm{H}), 9.49(\mathrm{t}, J=3.1 \mathrm{~Hz}, 1 \mathrm{H}) \mathrm{ppm}$.

${ }^{13} \mathrm{C}$ NMR $\left(125 \mathrm{MHz}, \mathrm{CD}_{2} \mathrm{Cl}_{2}\right): \delta=26.6,30.1,35.1,54.3,124.6,132.7,203.7$ ppm. 


\subsubsection{2-(1-Methylcyclohexa-2,5-dien-1-yl)ethan-1-ol (S5)}<smiles>CC1(CCO)C=CCC=C1</smiles>

S5

$\mathrm{C}_{9} \mathrm{H}_{14} \mathrm{O}$

$138.21 \mathrm{~g} / \mathrm{mol}$

A suspension of $\mathrm{NaBH}_{4}(890 \mathrm{mg}, 23.5 \mathrm{mmol}, 2.0$ equiv) in THF (50 mL) was cooled to $0{ }^{\circ} \mathrm{C}$. A solution of 2-(1-methylcyclohexa-2,5-dien-1-yl)acetaldehyde (S4, $1.60 \mathrm{~g}$, $11.7 \mathrm{mmol})$ in THF (15 mL) was added dropwise over $30 \mathrm{~min}$. The suspension was stirred overnight warming slowly to RT. At $0{ }^{\circ} \mathrm{C}$, the reaction was carefully quenched with $\mathrm{HCl}(1 \mathrm{M}$, aq.) before being diluted with brine $(100 \mathrm{~mL})$ and extracted with tertbutyl methyl ether $(3 \times 100 \mathrm{~mL})$. The combined organic phases were dried over $\mathrm{MgSO}_{4}$ and concentrated under reduced pressure. The residue was purified by flash column chromatography on silica gel using $n$-pentane: $\mathrm{Et}_{2} \mathrm{O}=5: 1$ as eluent to afford 2-(1-methylcyclohexa-2,5-dien-1-yl)ethan-1-ol (S5, $1.50 \mathrm{~g}, 93 \%)$ as a colorless oil.

$\mathbf{R}_{\mathbf{f}}=0.60$ (n-pentane:Et $\left.{ }_{2} \mathrm{O}=5: 1\right)$. IR $(\mathrm{ATR}): \tilde{v}=707,737,943,1006,1051,1363$, 1421, 1452, 2857, 2924, 3012, $3324 \mathrm{~cm}^{-1}$. HRMS (APCl) for $\mathrm{C}_{9} \mathrm{H}_{14} \mathrm{O}^{+}\left[\mathrm{M}^{+} \mathrm{H}^{+}\right]$: calculated 139.1123, found 139.1115. ${ }^{1} \mathrm{H}$ NMR (400 MHz, $\left.\mathrm{CD}_{2} \mathrm{Cl}_{2}\right): \delta=1.04(\mathrm{~s}, 3 \mathrm{H})$, 1.59 (t, $J=6.7 \mathrm{~Hz}, 2 \mathrm{H}), 2.60-2.62(\mathrm{~m}, 2 \mathrm{H}), 3.55$ (t, $J=6.7 \mathrm{~Hz}, 2 \mathrm{H}), 5.49$ (dt, $J=10.3$, $2.2 \mathrm{~Hz}, 2 \mathrm{H}), 5.70-5.75(\mathrm{~m}, 2 \mathrm{H}) \mathrm{ppm} .{ }^{13} \mathrm{C} \mathrm{NMR}\left(100 \mathrm{MHz}, \mathrm{CD}_{2} \mathrm{Cl}_{2}\right): \delta=26.6,30.6$, 35.6, 45.6, 61.6, 123.9, $134.2 \mathrm{ppm}$ 


\subsubsection{3-(2-Bromoethyl)-3-methylcyclohexa-1,4-diene (1)}

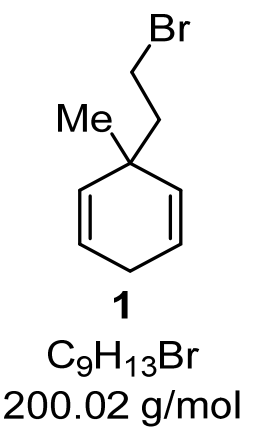

To a solution of $\mathrm{CBr}_{4}$ (260 mg, $0.800 \mathrm{mmol}, 1.1$ equiv) 2-(1-methylcyclohexa-2,5dien-1-yl)ethan-1-ol (S5, $100 \mathrm{mg}, 0.720 \mathrm{mmol})$ in $\mathrm{CH}_{2} \mathrm{Cl}_{2}(1 \mathrm{~mL})$ was added a solution of $\mathrm{Ph}_{3} \mathrm{P}$ (210 mg, $0.800 \mathrm{mmol}, 1.1$ equiv) in $\mathrm{CH}_{2} \mathrm{Cl}_{2}(1 \mathrm{~mL})$ dropwise at $0{ }^{\circ} \mathrm{C}$. The reaction was stirred overnight and allowed to slowly warm to RT. The solution was concentrated and then $n$-pentane $(50 \mathrm{~mL})$ was added to the residue to give white participate. After the removal of participate through filtration, the solution was concentrated under reduced pressure. The residue was purified by flash column chromatography on silica gel using $n$-pentane as eluent to afford 3-(2-bromoethyl)-3methylcyclohexa-1,4-diene $(1,115 \mathrm{mg}, 85 \%)$ as a colorless liquid.

$\mathbf{R}_{\mathbf{f}}=0.80$ (n-pentane). IR (ATR): $\tilde{v}=707,942,1144,1217,1452,2958 \mathrm{~cm}^{-1}$. HRMS (APCl) for $\mathrm{C}_{9} \mathrm{H}_{12} \mathrm{Br}^{+}\left[\mathrm{M}-\mathrm{H}^{+}\right]$: calculated 199.0117, found 199.0116. ${ }^{1} \mathrm{H}$ NMR (500 $\left.\mathrm{MHz}, \mathrm{CD}_{2} \mathrm{Cl}_{2}\right): \delta=1.06(\mathrm{~s}, 3 \mathrm{H}), 1.86-1.90(\mathrm{~m}, 2 \mathrm{H}), 2.50-2.64(\mathrm{~m}, 2 \mathrm{H}), 3.20-3.27(\mathrm{~m}$, $2 \mathrm{H}), 5.41(\mathrm{dt}, J=10.4,2.2 \mathrm{~Hz}, 2 \mathrm{H}), 5.76(\mathrm{dt}, J=10.4,3.3 \mathrm{~Hz}, 2 \mathrm{H}) \mathrm{ppm} .{ }^{13} \mathrm{C}$ NMR $\left(125 \mathrm{MHz}, \mathrm{CD}_{2} \mathrm{Cl}_{2}\right): \delta=26.7,30.2,30.6,37.7,46.4,124.89,132.6$ ppm. 
2.2 Synthesis of the 3-(2-bromoethyl)-1,3,5-trimethylcyclohexa-1,4-diene (2)

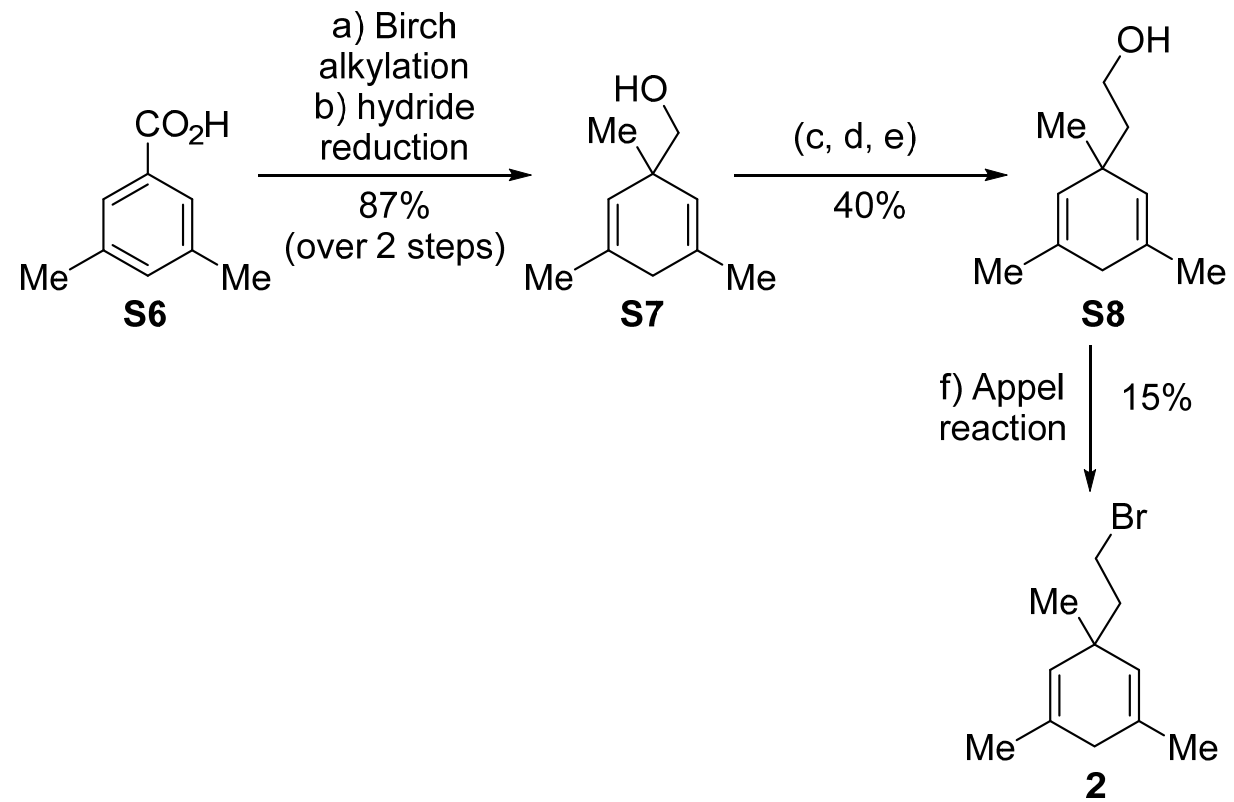

Scheme S2. Synthesis of $\mathrm{HBr}$ surrogate 2. Reaction conditions: a) $\mathrm{Na}$ (4.0 equiv), $t$-BuOH (1.0 equiv), $\mathrm{NH}_{3},-78{ }^{\circ} \mathrm{C}, 1 \mathrm{~h}$; Mel (4.0 equiv), THF, $-78{ }^{\circ} \mathrm{C}$ to RT, overnight; b) $\mathrm{LiAlH}_{4}\left(2.0\right.$ equiv), THF, $0{ }^{\circ} \mathrm{C}$; c) $(\mathrm{COCl})_{2}$ (1.5 equiv), DMSO (2.0 equiv), $\mathrm{CH}_{2} \mathrm{Cl}_{2}$, $-78{ }^{\circ} \mathrm{C}, 30 \mathrm{~min}$; $\mathrm{Et}_{3} \mathrm{~N}$ (excess), $-78{ }^{\circ} \mathrm{C}$ to $\mathrm{RT}, 30 \mathrm{~min}$; d) $\left[\mathrm{Ph}_{3} \mathrm{PCH}_{2} \mathrm{OMe}^{+} \mathrm{Cl}^{-}(1.2\right.$ equiv), $n$-BuLi (2.5 M in hexanes, 1.1 equiv), $0{ }^{\circ} \mathrm{C}$ to $\mathrm{RT}$, overnight; $\mathrm{HCl}$ in $\mathrm{H}_{2} \mathrm{O}$ (32\%), $0{ }^{\circ} \mathrm{C}, 2 \mathrm{~h}$; e) $\mathrm{NaBH}_{4}$ (2.0 equiv), THF, $0{ }^{\circ} \mathrm{C}$, overnight; f) $\mathrm{Ph}_{3} \mathrm{P}$ (1.1 equiv), $\mathrm{CBr}_{4}$ (1.1 equiv), $\mathrm{CH}_{2} \mathrm{Cl}_{2}, 0^{\circ} \mathrm{C}$ to $\mathrm{RT}$, overnight. 


\subsection{1 (1,3,5-Trimethylcyclohexa-2,5-dien-1-yl)methanol (S7)}

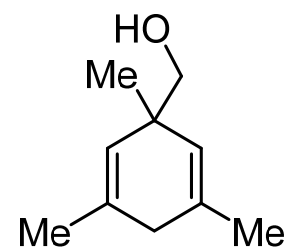

57

$\mathrm{C}_{10} \mathrm{H}_{16} \mathrm{O}$

$152.12 \mathrm{~g} / \mathrm{mol}$

A three-necked round-bottomed flask equipped with a dry ice condenser and an overhead stirrer was purged with $\mathrm{N}_{2}$ for 15 min before being placed in a $-78{ }^{\circ} \mathrm{C}$ dry ice/acetone bath. Ammonia (approx. $50 \mathrm{~mL}$ ) was condensed, and a solution of 3,5dimethylbenzoic acid (S6, $1.00 \mathrm{~g}, 6.67 \mathrm{mmol})$ and $t$-BuOH $(0.700 \mathrm{~mL}, 553 \mathrm{mg}, 7.47$ mmol, 1.1 equiv) in THF (10 mL) was then added dropwise. Sodium (600 mg, 26.0 mmol, 4.0 equiv) was added portionwise over a period of $30 \mathrm{~min}$ to give a dark green suspension. The dark green suspension was stirred at $-78{ }^{\circ} \mathrm{C}$ for a further $60 \mathrm{~min}$. A solution of methyl iodide (1.70 mL, $3.90 \mathrm{~g}, 27.4 \mathrm{mmol}, 4.0$ equiv) in THF (10 mL) was added dropwise over a period of $60 \mathrm{~min}$. The resulting pale yellow suspension was stirred overnight to facilitate the evaporation of ammonia. Water $(20 \mathrm{~mL})$ was added, and the aqueous phase was acidified with $\mathrm{HCl}(32 \%$, aq.) until $\mathrm{pH}=3$ was reached, before being extracted with tert-butyl methyl ether $(3 \times 50 \mathrm{~mL})$. The combined organic phases were washed with brine $(50 \mathrm{~mL})$, dried over $\mathrm{MgSO}_{4}$ and concentrated under reduced pressure to afford 1,3,5-trimethylcyclohexa-2,5-diene-1-carboxylic acid as a light yellow oil.

Without further purification, a solution of crude 1,3,5-trimethylcyclohexa-2,5-diene-1carboxylic acid in THF (10 mL) was added dropwise to a suspension of $\mathrm{LiAlH}_{4}(500$ $\mathrm{mg}, 13.1 \mathrm{mmol}, 2.0$ equiv) in THF $(10 \mathrm{~mL})$ at $0{ }^{\circ} \mathrm{C}$. The resulting mixture was stirred for 4 hours at $0{ }^{\circ} \mathrm{C}$ before being carefully quenched with $\mathrm{MeOH}$. $\mathrm{HCl}(1 \mathrm{M}$, aq.) was then added until the suspension disappeared. The aqueous phase was extracted with tert-butyl methyl ether $(3 \times 50 \mathrm{~mL})$, and the combined organic phases were washed with brine $(50 \mathrm{~mL})$ and concentrated under reduced pressure. The residue was purified by flash column chromatography on silica gel using cyclohexane:tertbutyl methyl ether $=20: 1$ as eluent to afford methyl (1,3,5-trimethylcyclohexa-2,5dien-1-yl)methanol (S7, $880 \mathrm{mg}, \mathbf{8 7 \%})$ as a yellow oil. 
$\mathbf{R}_{\mathbf{f}}=0.52$ (cyclohexane:tert-butyl methyl ether = 2:1). IR $(A T R): \tilde{v}=825,926,1383$, 1436, 2859, 2914, 2962, $3344 \mathrm{~cm}^{-1} .{ }^{1} \mathrm{H}$ NMR (400 MHz, $\left.\mathrm{C}_{6} \mathrm{D}_{6}\right): \delta=0.97$ (s, 3H), 1.54 (br s, 1H), 1.59 (s, 6H), 214-2.28 (m, 2H), 3.27 (s, 2H), $5.16(\mathrm{~s}, 2 \mathrm{H})$ ppm. ${ }^{13} \mathrm{C}$ NMR $\left(100 \mathrm{MHz}, \mathrm{C}_{6} \mathrm{D}_{6}\right): \delta=23.0,25.1,36.4,41.4,71.5,126.5,132.3 \mathrm{ppm}$. 


\subsubsection{2-(1,3,5-Trimethylcyclohexa-2,5-dien-1-yl)ethan-1-ol (S8)}

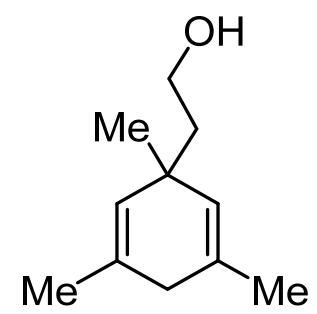

S8

$\mathrm{C}_{11} \mathrm{H}_{18} \mathrm{O}$

$166.14 \mathrm{~g} / \mathrm{mol}$

To a solution of oxalyl chloride (2 $\mathrm{M}$ in $\mathrm{CH}_{2} \mathrm{Cl}_{2}, 2.50 \mathrm{~mL}, 5.00 \mathrm{mmol}, 1.5$ equiv) at $-78{ }^{\circ} \mathrm{C}$ was added DMSO $(0.500 \mathrm{~mL}, 550 \mathrm{mg}, 7.05 \mathrm{mmol}, 2.1$ equiv). The mixture was stirred for $10 \mathrm{~min}$ at $-78{ }^{\circ} \mathrm{C}$ after which a solution of afford methyl $(1,3,5-$ trimethylcyclohexa-2,5-dien-1-yl)methanol (S7, $500 \mathrm{mg}, 3.29 \mathrm{mmol})$ in $\mathrm{CH}_{2} \mathrm{Cl}_{2}(5 \mathrm{~mL})$ was added dropwise over $10 \mathrm{~min}$. Following this, the reaction was stirred for $20 \mathrm{~min}$ at $-78{ }^{\circ} \mathrm{C}$ and then $\mathrm{Et}_{3} \mathrm{~N}(2 \mathrm{~mL})$ was added dropwise. The reaction was allowed to warm to RT over $30 \mathrm{~min}$ and $\mathrm{NH}_{4} \mathrm{Cl}$ (sat., aq., $30 \mathrm{~mL}$ ) was then added. The aqueous phase was extracted with $\mathrm{Et}_{2} \mathrm{O}(3 \times 30 \mathrm{~mL})$, and the combined organic phases were washed with brine $(30 \mathrm{~mL})$. After drying over $\mathrm{MgSO}_{4}$, all solvents were removed under reduced pressure to afford 1,3,5-trimethylcyclohexa-2,5-diene-1-carbaldehyde which was used directly without further purification.

A suspension of $\mathrm{Ph}_{3} \mathrm{PCH}_{2} \mathrm{OMeCl}$ (1.35 g, 4.00 mmol, 1.2 equiv) in THF (30 mL) was cooled to $0{ }^{\circ} \mathrm{C}$. $t$-BuOK (448 mg, $4.00 \mathrm{mmol}, 1.1$ equiv) was added dropwise over a period of $10 \mathrm{~min}$. After a further $60 \mathrm{~min}$ at $0{ }^{\circ} \mathrm{C}$, a solution of $1,3,5-$ trimethylcyclohexa-2,5-diene-1-carbaldehyde in THF $(5 \mathrm{~mL})$ was added dropwise and the reaction mixture was stirred overnight, warming slowly to RT. The reaction was cooled to $0{ }^{\circ} \mathrm{C}$ and $\mathrm{HCl}(32 \%$, aq., $5 \mathrm{~mL})$ was added dropwise. The reaction was stirred for 2 hours before being diluted with tert-butyl methyl ether (30 $\mathrm{mL})$, and neutralized by portionwise addition of $\mathrm{K}_{2} \mathrm{CO}_{3}$ powder. Filtration gave a solution of 2(1,3,5-trimethylcyclohexa-2,5-dien-1-yl)acetaldehyde which was concentrated to approx. $10 \mathrm{~mL}$ and used directly in the next step.

A suspension of $\mathrm{NaBH}_{4}(250 \mathrm{mg}, 6.60 \mathrm{mmol}, 2.0$ equiv) in THF (10 mL) was cooled to $0{ }^{\circ} \mathrm{C}$. The solution of of 2-(1,3,5-trimethylcyclohexa-2,5-dien-1-yl)acetaldehyde was added dropwise over a period of $30 \mathrm{~min}$. The suspension was stirred overnight, 
warming slowly to RT. The reaction was cooled to $0{ }^{\circ} \mathrm{C}$ and carefully quenched with $\mathrm{HCl}(1 \mathrm{M}$, aq. $10 \mathrm{ml})$ before being diluted with brine $(50 \mathrm{~mL})$ and extracted with tertbutyl methyl ether $(3 \times 50 \mathrm{~mL})$. The combined organic phases were dried over $\mathrm{MgSO}_{4}$ and concentrated under reduced pressure. The residue was purified by flash column chromatography on silica gel using $n$-pentane: $\mathrm{Et}_{2} \mathrm{O}=5: 1$ as eluent to afford 2-(1,3,5-trimethylcyclohexa-2,5-dien-1-yl)ethan-1-ol (\$8, $200 \mathrm{mg}, 40 \%)$ as a colorless oil.

$\mathbf{R}_{\mathbf{f}}=0.60$ (n-pentane:Et $\left.\mathrm{E}_{2} \mathrm{O}=2: 1\right)^{1} \mathrm{H}$ NMR $\left(400 \mathrm{MHz}, \mathrm{C}_{6} \mathrm{D}_{6}\right): \delta=1.00(\mathrm{~s}, 3 \mathrm{H}), 1.46(\mathrm{br}$ $\mathrm{s}, 1 \mathrm{H}), 1.51-1.55(\mathrm{~m}, 8 \mathrm{H}), 2.10-2.20(\mathrm{~m}, 2 \mathrm{H}), 3.53(\mathrm{t}, J=6.7 \mathrm{~Hz}, 2 \mathrm{H}), 5.11(\mathrm{~s}, 2 \mathrm{H})$ ppm. ${ }^{13} \mathrm{C}$ NMR $\left(100 \mathrm{MHz}, \mathrm{C}_{6} \mathrm{D}_{6}\right): \delta=22.8,30.5,36.0,37.7,45.9,60.9,128.8,130.3$ ppm. 


\subsubsection{3-(2-Bromoethyl)-1,3,5-trimethylcyclohexa-1,4-diene (2)}<smiles>CC1=CC(C)(CCBr)C=C(C)C1</smiles>

2

$\mathrm{C}_{11} \mathrm{H}_{17} \mathrm{Br}$

$228.05 \mathrm{~g} / \mathrm{mol}$

To a solution of $\mathrm{CBr}_{4}(220 \mathrm{mg}, 0.660 \mathrm{mmol}, 1.1$ equiv) 2-(1,3,5-trimethylcyclohexa2,5-dien-1-yl)ethan-1-ol (S8, $100 \mathrm{mg}, 0.602 \mathrm{mmol}$ ) in $\mathrm{CH}_{2} \mathrm{Cl}_{2}(1 \mathrm{~mL})$ was added a solution of $\mathrm{Ph}_{3} \mathrm{P}\left(170 \mathrm{mg}, 0.660 \mathrm{mmol}, 1.1\right.$ equiv) in $\mathrm{CH}_{2} \mathrm{Cl}_{2}(1 \mathrm{~mL})$ dropwise at $0{ }^{\circ} \mathrm{C}$. The reaction was stirred overnight and allowed to slowly warm to RT. The solution was concentrated and then hexane $(50 \mathrm{~mL})$ was added to the residue to give white participate. After the removal of participate through filtration, the solution was concentrated under reduced pressure. The residue was purified by flash column chromatography on silica gel using cyclohexane as eluent to afford 3-(2-bromoethyl)1,3,5-trimethylcyclohexa-1,4-diene (2, $20 \mathrm{mg}, 15 \%)$ as a colorless liquid.

$\mathbf{R}_{\mathbf{f}}=0.60$ (cyclohexane). IR (ATR): $\tilde{V}=829,1439,2358,2923 \mathrm{~cm}^{-1}$. HRMS (APCI) for $\mathrm{C}_{11} \mathrm{H}_{17} \mathrm{Br}^{+}\left[\mathrm{M}^{+}\right]$: calculated 228.0514, found 228.0512. ${ }^{1} \mathrm{H}$ NMR (400 MHz, $\left.\mathrm{C}_{6} \mathrm{D}_{6}\right)$ : $\delta=0.88(\mathrm{~s}, 3 \mathrm{H}), 1.49(\mathrm{~s}, 6 \mathrm{H}), 1.75-1.80(\mathrm{~m}, 2 \mathrm{H}), 1.95-2.10(\mathrm{~m}, 2 \mathrm{H}), 3.05-3.09(\mathrm{~m}$, $2 \mathrm{H}), 4.83$ (s, 2H) ppm. ${ }^{13} \mathrm{C}$ NMR (125 MHz, $\left.\mathrm{C}_{6} \mathrm{D}_{6}\right): \delta=22.8,30.0,30.5,36.0,39.7$, 46.6, 122.1, $131.3 \mathrm{ppm}$. 


\subsection{Synthesis of the 1-(2-bromoethyl)-1,4-dihydro-1,1'-biphenyl (3)}

\section{Method A:}<smiles>Pc1ccccc1</smiles>

S9 a) $\mathrm{NH}_{3(\text { (I) }} / \mathrm{THF}$,

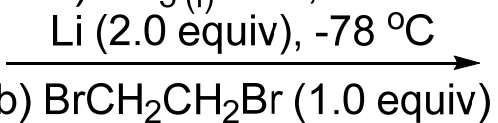
$66 \%$<smiles>BrCCC1(c2ccccc2)C=CCC=C1</smiles>

3

Scheme S3. Preparation of surrogate 3 in one step

\subsubsection{1-(2-Bromodoethyl)-1,4-dihydro-1,1'-biphenyl (3)}

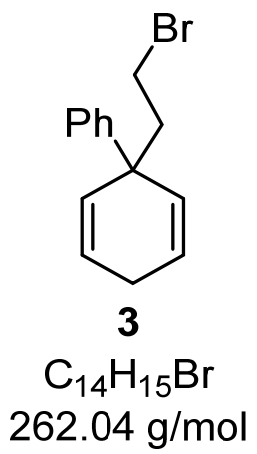

A three-necked round-bottomed flask equipped with a dry ice condenser and an overhead stirrer was purged with $\mathrm{N}_{2}$ for 15 min before being placed in a dry ice/acetone bath. Ammonia (approx. $50 \mathrm{~mL}$ ) was condensed, and then lithium (160 mg, $22.8 \mathrm{mmol}, 2.0$ equiv) was added portion-wise over a period of $15 \mathrm{~min}$ to result in a dark blue suspension. A solution of biphenyl $(\mathbf{S 9}, 1.80 \mathrm{~g}, 11.6 \mathrm{mmol})$ in THF $(5 \mathrm{~mL})$ was added dropwise over a period of $30 \mathrm{~min}$ to result in a brick red suspension. The suspension was stirred at $-78{ }^{\circ} \mathrm{C}$ for a further $15 \mathrm{~min}$. Neat $\mathrm{BrCH}_{2} \mathrm{CH}_{2} \mathrm{Br}(1.00 \mathrm{~mL}$, $2.18 \mathrm{~g}, 11.6 \mathrm{mmol}, 1.0$ equiv) was then added fast. The resulting pale yellow suspension was stirred overnight to facilitate the evaporation of ammonia. Water (50 $\mathrm{mL})$ was added and the mixture was extracted with tert-butyl methyl ether $(3 \times 50$ $\mathrm{mL})$. The combined organic phases were washed with brine $(100 \mathrm{~mL})$, dried over $\mathrm{MgSO}_{4}$ and concentrated under reduced pressure. The residue was purified by flash column chromatography on silica gel using cyclohexane:tert-butyl methyl ether $=50: 1$ as eluent to afford 1-(2-bromoethyl)-1,4-dihydro-1,1'-biphenyl (3, $1.90 \mathrm{~g}, 66 \%)$ as a colorless oil. 
$\mathbf{R}_{\mathbf{f}}=0.60$ (cyclohexane). IR (ATR): $\tilde{v}=692,732,762,945,1443,1491,2812,3018$ $\mathrm{cm}^{-1}$. HRMS (APCl) for $\mathrm{C}_{14} \mathrm{H}_{15} \mathrm{Br}^{+}\left[\mathrm{M}^{+}\right]$: calculated 262.0357, found 262.0355 . ${ }^{1} \mathrm{H}$ NMR $\left(500 \mathrm{MHz}, \mathrm{CD}_{2} \mathrm{Cl}_{2}\right): \delta=2.39-2.43(\mathrm{~m}, 2 \mathrm{H}), 2.63-2.76(\mathrm{~m}, 2 \mathrm{H}), 3.37-3.41(\mathrm{~m}$, 2H), $5.62(\mathrm{dt}, J=10.7,2.0 \mathrm{~Hz}, 2 \mathrm{H}$ ), $5.93(\mathrm{dt}, J=10.7,3.4 \mathrm{~Hz}, 1 \mathrm{H}), 7.20(\mathrm{tt}, J=7.0$, $2.0 \mathrm{~Hz}, 1 \mathrm{H}), 7.31-7.36(\mathrm{~m}, 4 \mathrm{H}) \mathrm{ppm} .{ }^{13} \mathrm{C}$ NMR $\left(125 \mathrm{MHz}, \mathrm{CD}_{2} \mathrm{Cl}_{2}\right): \delta=26.4,30.3$, $43.7,45.1,125.2,126.6,126.7,128.8,131.3,147.5 \mathrm{ppm}$.

\section{Method B:}<smiles>Pc1ccccc1</smiles>

S9

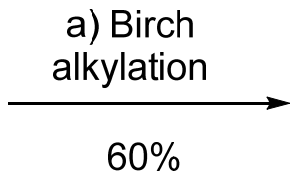

$60 \%$

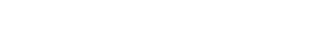

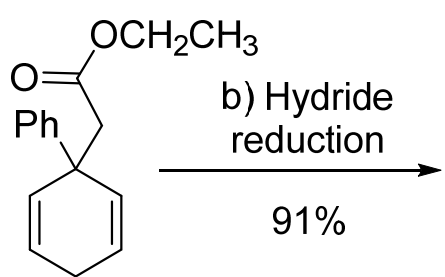

S10<smiles>OCCC1(c2ccccc2)C=CCC=C1</smiles>

S11

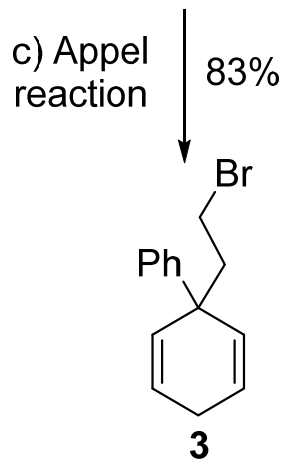

Scheme S4. Synthesis of $\mathrm{HBr}$ surrogate 3. Reaction conditions: a) Li (4.0 equiv), $\mathrm{NH}_{3},-78{ }^{\circ} \mathrm{C}$, 1 hour; $\mathrm{BrCH}_{2}$ COOEt (4.0 equiv), $-78{ }^{\circ} \mathrm{C}$ to RT, overnight; b) $\mathrm{LiAlH}_{4}$ (2.0 equiv), THF, $0{ }^{\circ} \mathrm{C}, 2.0 \mathrm{~h}$; c) $\mathrm{Ph}_{3} \mathrm{P}$ (1.1 equiv), $\mathrm{CBr}_{4}$ (1.1 equiv), $\mathrm{CH}_{2} \mathrm{Cl}_{2}, 0{ }^{\circ} \mathrm{C}$ to RT, overnight. 


\subsubsection{Ethyl 2-([1,1'-biphenyl]-1(4H)-yl)acetate (S10)}

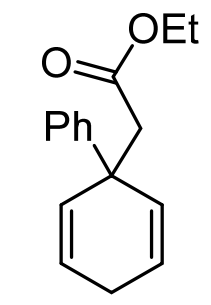

S10

$\mathrm{C}_{16} \mathrm{H}_{18} \mathrm{O}_{2}$

$242.32 \mathrm{~g} / \mathrm{mol}$

A three-necked round-bottomed flask equipped with a dry ice condenser and an overhead stirrer was purged with $\mathrm{N}_{2}$ for 15 min before being placed in a dry ice/acetone bath. Ammonia (approx. $100 \mathrm{~mL}$ ) was condensed, and then lithium (750 $\mathrm{mg}, 107 \mathrm{mmol}, 4.0$ equiv) was added portionwise over a period of $30 \mathrm{~min}$ to result in a dark blue suspension. A solution of biphenyl (S9, $4.00 \mathrm{~g}, 26.0 \mathrm{mmol})$ in THF (10 $\mathrm{mL}$ ) was added dropwise over a period of $30 \mathrm{~min}$ to result in a brick red suspension. The suspension was stirred at $-78{ }^{\circ} \mathrm{C}$ for a further $60 \mathrm{~min}$. A solution of $\mathrm{BrCH}_{2} \mathrm{COOEt}$ (11.0 mL, $17.3 \mathrm{~g}, 100 \mathrm{mmol}, 2.0$ equiv) in THF $(20 \mathrm{~mL})$ was then added dropwise over a period of $60 \mathrm{~min}$. The resulting pale yellow suspension was stirred overnight to facilitate the evaporation of ammonia. Water $(50 \mathrm{~mL})$ was added and the mixture was extracted with tert-butyl methyl ether $(3 \times 50 \mathrm{~mL})$. The combined organic phases were washed with brine $(50 \mathrm{~mL})$, dried over $\mathrm{MgSO}_{4}$ and concentrated under reduced pressure. The residue was purified by flash column chromatography on silica gel using cyclohexane:tert-butyl methyl ether $=20: 1$ as eluent to afford ethyl 2-([1,1'biphenyl]-1(4H)-yl)acetate (\$10, $3.80 \mathrm{~g}, 60 \%)$ as a colorless oil.

$\mathbf{R}_{\mathbf{f}}=0.60$ (cyclohexane:tert-butyl methyl ether = 10:1). IR $($ ATR): $\tilde{v}=694,762,1029$, 1151, 1231, 1312, 1728, $2928 \mathrm{~cm}^{-1}$. HRMS (APCI) for $\mathrm{C}_{16} \mathrm{H}_{19} \mathrm{O}_{2}{ }^{+}\left[\mathrm{M}+\mathrm{H}^{+}\right]$: calculated 243.1380, found 243.1378. ${ }^{1} \mathrm{H}$ NMR (400 MHz, $\left.\mathrm{C}_{6} \mathrm{D}_{6}\right): \delta=0.89(\mathrm{t}, J=7.0 \mathrm{~Hz}, 3 \mathrm{H})$, 2.31-2.47 (m, 2H), $2.78(\mathrm{~s}, 2 \mathrm{H}), 3.87(\mathrm{q}, J=7.0 \mathrm{~Hz}, 2 \mathrm{H}), 5.65$ (dt, $J=10.7,3.3 \mathrm{~Hz}$, 2H), $5.93(\mathrm{dt}, J=10.7,2.2 \mathrm{~Hz}, 2 \mathrm{H}), 7.03(\mathrm{t}, J=7.0 \mathrm{~Hz}, 1 \mathrm{H}), 7.14-7.18(\mathrm{~m}, 2 \mathrm{H}), 7.03$ (d, $J=7.3 \mathrm{~Hz}, 2 \mathrm{H})$ ppm. ${ }^{13} \mathrm{C}$ NMR $\left(100 \mathrm{MHz}, \mathrm{C}_{6} \mathrm{D}_{6}\right): \delta=14.2,26.1,42.9,48.0,59.9$, 123.6, 126.4, 128.6, 132.4, 147.0, 170.1 ppm. 


\subsubsection{2-([1,1'-Biphenyl]-1(4H)-yl)ethan-1-ol (S11)}<smiles>OCCC1(c2ccccc2)C=CCC=C1</smiles>

S11

$\mathrm{C}_{14} \mathrm{H}_{16} \mathrm{O}$

$200.12 \mathrm{~g} / \mathrm{mol}$

A solution of ethyl 2-([1,1'-biphenyl]-1(4H)-yl)acetate $(\mathbf{S 1 0}, 3.80 \mathrm{~g}, 15.7 \mathrm{mmol})$ in THF $(10 \mathrm{~mL})$ was added dropwise to a suspension of $\mathrm{LiAlH}_{4}(1.12 \mathrm{~g}, 30.0 \mathrm{mmol}, 2.0$ equiv) in THF $(10 \mathrm{~mL})$ at $0{ }^{\circ} \mathrm{C}$. The resulting mixture was stirred for 4 hours at $0{ }^{\circ} \mathrm{C}$ before being carefully quenched with $\mathrm{MeOH}$. $\mathrm{HCl}(1 \mathrm{M}$, aq.) was then added until the suspension disappeared. The aqueous phase was extracted with tert-butyl methyl ether $(3 \times 100 \mathrm{~mL})$, and the combined organic phases were washed with brine $(100$ $\mathrm{mL}$ ) and concentrated under reduced pressure. The residue was purified by flash column chromato-graphy on silica gel using cyclohexane:tert-butyl methyl ether = 20:1 as eluent to afford the 2-([1,1'-biphenyl]-1(4H)-yl)ethan-1-ol (S11, $2.80 \mathrm{~g}, 90 \%)$ as a white solid.

M.P. = $61-63{ }^{\circ} \mathrm{C}$ (cyclohexane). $\mathbf{R}_{\mathbf{f}}=0.17$ (cyclohexane:tert-butyl methyl ether = 10:1). IR (ATR): $\tilde{v}=693,736,943,1020,1338,1445,2861,2933,3013,3350 \mathrm{~cm}^{-1}$. HRMS (EI) for $\mathrm{C}_{14} \mathrm{H}_{14} \mathrm{O}^{+}\left[\mathrm{M}^{+}\right]$: calculated 200.2810, found 200.2809. ${ }^{1} \mathrm{H}$ NMR (400 $\left.\mathrm{MHz}, \mathrm{CDCl}_{3}\right): \delta=2.03(\mathrm{t}, J=7.0 \mathrm{~Hz}, 2 \mathrm{H}), 2.16(\mathrm{br}, 1 \mathrm{H}), 2.57-2.60(\mathrm{~m}, 2 \mathrm{H}), 3.62(\mathrm{t}, J$ $=7.0 \mathrm{~Hz}, 2 \mathrm{H}), 5.56(\mathrm{dt}, J=10.6,2.2 \mathrm{~Hz}, 2 \mathrm{H}), 5.74-5.79(\mathrm{~m}, 2 \mathrm{H}), 7.06-7.11(\mathrm{~m}, 1 \mathrm{H})$, 7.18-7.27 (m, 4H) ppm. ${ }^{13} \mathrm{C}$ NMR (100 MHz, $\left.\mathrm{CDCl}_{3}\right): \delta=25.9,42.2,42.7,60.6$, $123.8,126.1,126.5,128.4,132.5,147.6 \mathrm{ppm}$. 


\subsubsection{1-(2-Bromodoethyl)-1,4-dihydro-1,1'-biphenyl (3)}

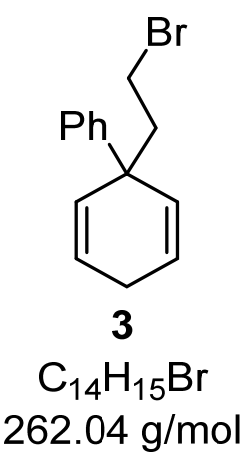

To a solution of $\mathrm{CBr}_{4}$ (4.23 g, $12.8 \mathrm{mmol}, 1.1$ equiv) and 2-(1-methylcyclohexa-2,5dien-1-yl)ethan-1-ol $(3,2.00 \mathrm{~g}, 10.0 \mathrm{mmol})$ in $\mathrm{CH}_{2} \mathrm{Cl}_{2}(5 \mathrm{~mL})$ was added a solution of $\mathrm{Ph}_{3} \mathrm{P}$ (3.33 g, $12.8 \mathrm{mmol}, 1.1$ equiv) in $\mathrm{CH}_{2} \mathrm{Cl}_{2}(5 \mathrm{~mL})$ dropwise at $0{ }^{\circ} \mathrm{C}$. The reaction was stirred overnight and allowed to slowly warm to RT. The solution was concentrated and then hexane $(50 \mathrm{~mL})$ was added to the residue to give white participate. After the removal of participate through filtration, the solution was concentrated under reduced pressure. The residue was purified by flash column chromatography on silica gel using cyclohexane as eluent to afford 3-(2-bromoethyl)3-methylcyclohexa-1,4-diene (3, $2.53 \mathrm{~g}, 83 \%)$ as a colorless oil. Note: 3-(2Bromoethyl)-3-methylcyclohexa-1,4-diene (3) slowly crystalized under $-30{ }^{\circ} \mathrm{C}$ in fridge.

$\mathbf{R}_{\mathbf{f}}=0.60$ (cyclohexane). IR (ATR): $\tilde{v}=692,732,762,945,1443,1491,2812,3018$ $\mathrm{cm}^{-1}$. HRMS $(\mathrm{APCl})$ for $\mathrm{C}_{14} \mathrm{H}_{15} \mathrm{Br}^{+}\left[\mathrm{M}^{+}\right]$: calculated 262.0357, found 262.0355. ${ }^{1} \mathrm{H}$ NMR (500 MHz, $\left.\mathrm{CD}_{2} \mathrm{Cl}_{2}\right): \delta=2.39-2.43(\mathrm{~m}, 2 \mathrm{H}), 2.63-2.76(\mathrm{~m}, 2 \mathrm{H}), 3.37-3.41(\mathrm{~m}$, 2H), 5.62 (dt, $J=10.7,2.0 \mathrm{~Hz}, 2 \mathrm{H}), 5.93(\mathrm{dt}, J=10.7,3.4 \mathrm{~Hz}, 1 \mathrm{H}), 7.20(\mathrm{tt}, J=7.0$, $2.0 \mathrm{~Hz}, 1 \mathrm{H}), 7.31-7.36(\mathrm{~m}, 4 \mathrm{H}) \mathrm{ppm} .{ }^{13} \mathrm{C}$ NMR (125 MHz, $\left.\mathrm{CD}_{2} \mathrm{Cl}_{2}\right): \delta=26.4,30.3$, $43.7,45.1,125.2,126.6,126.7,128.8,131.3,147.5$ ppm. 


\section{Catalytic Transfer Hydrobromination}

\subsection{General procedures for catalytic transfer hydrobromination}

Method A: In a glovebox, a pressure tube is charged with a solution of the indicated alkyne and 1-(2-bromoethyl)-1,4-dihydro-1,1'-biphenyl (3) in toluene (1.5 mL), and a solution of 4-toluenesulfonic acid $(20 \mathrm{~mol} \%)$ in toluene $(0.5 \mathrm{~mL})$ is added. The tube is sealed and removed from the glovebox and stirred in a preheated $140{ }^{\circ} \mathrm{C}$ oil bath for 24 to 36 hours. The reaction was cooled to RT and directly purified by flash column chromatography on silica gel without prior concentration.

Method B: In a glovebox, a pressure tube is charged with a solution of the indicated alkyne and 1-(2-bromoethyl)-1,4-dihydro-1,1'-biphenyl (3) in chlorobenzene (2.0 mL), and a solution of $\mathrm{Tf}_{2} \mathrm{NH}(20 \mathrm{~mol} \%)$ in chlorobenzene $(0.5 \mathrm{~mL})$ is added. The tube is sealed and removed from the glove box and stirred in a preheated $160{ }^{\circ} \mathrm{C}$ bath for 72 hours. The reaction was cooled to RT and directly purified by flash column chromatography on silica gel without prior concentration. 


\subsection{Substrate scope with unactivated alkynes}<smiles>C=C(Br)c1ccccc1C</smiles>

5b: $48 \%$<smiles>C=C(Br)c1ccc(B=O)cc1</smiles>

5c: $54 \%$<smiles>C/C=C(\Br)c1ccccc1</smiles>

5d: $75 \%$

$$
\begin{gathered}
Z: E=83: 17 \\
85 \% \\
Z: E=95: 5
\end{gathered}
$$<smiles>C=C(Br)c1ccc(C(=O)O)cc1</smiles>

5f: $75 \%$

Scheme S5. Transfer hydrobromination of unactivated alkynes

\subsubsection{1-(1-Bromovinyl)-2-methylbenzene (5b)}<smiles>C=C(Br)c1ccccc1C</smiles>

$5 b$

$\mathrm{C}_{9} \mathrm{H}_{9} \mathrm{Br}$

$195.99 \mathrm{~g} / \mathrm{mol}$

Prepared from 1-ethynyl-2-methylbenzene (4b, $20 \mathrm{mg}, 0.17 \mathrm{~mol})$ and surrogate 3 (60 $\mathrm{mg}, 0.23 \mathrm{mmol}, 1.3$ equiv) according to Method A, over 48 hours. The residue was purified by flash column chromatography on silica gel using $n$-pentane as the eluent to afford 1-(1-bromovinyl)-2-methylbenzene (5b, $16 \mathrm{mg}, 48 \%$ ) as a colorless oil. Note: Due to product volatility, samples should not be left under vacuum for extended periods of time.

$\mathbf{R}_{\mathbf{f}}=0.90$ (n-pentane). IR (ATR): $\tilde{v}=764,896,1215,1454,1628,2922,3019 \mathrm{~cm}^{-1}$. HRMS (El) for $\mathrm{C}_{9} \mathrm{H}_{9} \mathrm{IBr}^{+}\left[\mathrm{M}^{+}\right]$: calculated 195.9888, found 195.9883. ${ }^{1} \mathrm{H}$ NMR (500 $\left.\mathrm{MHz}, \mathrm{CD}_{2} \mathrm{Cl}_{2}\right): \delta=2.40(\mathrm{~s}, 3 \mathrm{H}), 6.74(\mathrm{~d}, J=1.7 \mathrm{~Hz}, 1 \mathrm{H}), 6.89(\mathrm{~d}, J=1.7 \mathrm{~Hz}, 1 \mathrm{H})$, 7.16-7.27 (m, 4H) ppm. ${ }^{13} \mathrm{C}$ NMR (125 MHz, $\left.\mathrm{CD}_{2} \mathrm{Cl}_{2}\right): \delta=19.9,121.3,126.1,129.2$, 129.3, 129.6, 130.7, 136.1, 140.8 ppm. 


\subsubsection{2-(4-(1-Bromovinyl)phenyl)-4,4,5,5-tetramethyl-1,3,2-dioxaborolane (5c)}<smiles>C=C(Br)c1ccc(B=O)cc1</smiles>

$5 c$

$\mathrm{C}_{14} \mathrm{H}_{18} \mathrm{BBrO}_{2}$

$308.06 \mathrm{~g} / \mathrm{mol}$

Prepared from 2-(4-ethynylphenyl)-4,4,5,5-tetramethyl-1,3,2-dioxaborolane (4c, 40 $\mathrm{mg}, 0.18 \mathrm{~mol}$ ) and surrogate 3 (60 $\mathrm{mg}, 0.23 \mathrm{mmol}, 1.3$ equiv) according to Method $\mathbf{A}$, over 48 hours. The residue was purified by flash column chromatography on silica gel using $n$-pentane: tert-butyl methyl ether $=10: 1$ as the eluent to afford 2-(4-(1bromovinyl)phenyl)-4,4,5,5-tetramethyl-1,3,2-dioxaborolane (5c, $30 \mathrm{mg}, 54 \%$ ) as a colorless viscous oil. Note: A long column is necessary to achieve adequate separation.

$\mathbf{R}_{\mathbf{f}}=0.50$ (n-pentane: tert-butyl methyl ether = 10:1). IR (ATR): $\tilde{v}=857,1088,1356$, 1606, 2977, $3291 \mathrm{~cm}^{-1}$. HRMS (EI) for $\mathrm{C}_{14} \mathrm{H}_{19} \mathrm{BBrO}_{2}^{+}\left[\mathrm{M}+\mathrm{H}^{+}\right]$: calculated 309.0656 , found 309.0654. ${ }^{1} \mathrm{H}$ NMR (500 MHz, $\left.\mathrm{CD}_{2} \mathrm{Cl}_{2}\right): \delta=1.36(\mathrm{~s}, 12 \mathrm{H}), 5.82(\mathrm{~d}, J=2.3 \mathrm{~Hz}$, $1 \mathrm{H}), 6.20(\mathrm{~d}, J=2.3 \mathrm{~Hz}, 1 \mathrm{H}), 7.59(\mathrm{~d}, J=7.0 \mathrm{~Hz}, 2 \mathrm{H}), 7.74(\mathrm{~d}, J=7.0 \mathrm{~Hz}, 2 \mathrm{H}) \mathrm{ppm}$. ${ }^{13} \mathrm{C}$ NMR $\left(125 \mathrm{MHz}, \mathrm{CD}_{2} \mathrm{Cl}_{2}\right): \delta=25.0,27.3,84.4,118.7,126.8,131.1,135.0,141.1$ ppm. 


\subsection{3 (1-Bromoprop-1-en-1-yl)benzene (5d)}<smiles>C/C=C(\Br)c1ccccc1</smiles>

Z-5d

$\mathrm{C}_{9} \mathrm{H}_{9} \mathrm{Br}$

$195.99 \mathrm{~g} / \mathrm{mol}$

On $1.0 \mathrm{mmol}$ scale:

According to a modified procedure from method $A$, in a glovebox, a pressure tube is charged with a solution of prop-1-yn-1-ylbenzene (4d, $120 \mathrm{mg}, 1.03 \mathrm{mmol}$ ) and surrogate 3 (520 mg, $198 \mathrm{mmol}, 1.9$ equiv) in toluene $(2.0 \mathrm{~mL})$, and a solution of 4toluenesulfonic acid (34 mg, $0.20 \mathrm{mmol}, 20 \mathrm{~mol} \%)$ in toluene $(1.0 \mathrm{~mL})$ is added. The tube is sealed and removed from the glove box and stirred in a preheated $140{ }^{\circ} \mathrm{C}$ bath for 24 hours. The reaction was cooled to RT and directly purified by flash column chromatography on silica gel without prior concentration using $n$-pentane as the eluent to afford (1-bromoprop-1-en-1-yl)benzene $(Z: E=83: 17)(5 \mathrm{~d}, 152 \mathrm{mg}$, $75 \%)$ as a colorless oil. Note: Biphenyl was isolated as the byproduct (S9, $280 \mathrm{mg}$, $91 \%$ ), which could be recycled for the preparation of surrogate 3 .

On $0.24 \mathrm{mmol}$ scale:

Prepared from 4 prop-1-yn-1-ylbenzene (4d, $28 \mathrm{mg}, 0.24 \mathrm{~mol}$ ) and surrogate 3 (60 $\mathrm{mg}, 0.23 \mathrm{mmol}, 1.0$ equiv) according to Method A, over 12 hours. The residue was purified by flash column chromatography on silica gel using $n$-pentane as the eluent to afford (1-bromoprop-1-en-1-yl)benzene $(Z: E=95: 5)(5 \mathbf{d}, 40 \mathrm{mg}, 85 \%)$ as a colorless oil.

The $Z$ configuration was determined by NOESY measurements.

$\mathbf{R}_{\mathbf{f}}=0.90$ (n-pentane). IR (ATR): $\tilde{v}=693,754,1235,1444,1687,2919 \mathrm{~cm}^{-1}$. HRMS (El) for $\mathrm{C}_{9} \mathrm{H}_{9} \mathrm{Br}^{+}\left[\mathrm{M}^{+}\right]$: calculated 195.9888, found 195.9883 .

For (Z)-(1-bromoprop-1-en-1-yl)benzene (Z-5d)

${ }^{1} \mathrm{H}$ NMR (400 MHz, $\left.\mathrm{C}_{6} \mathrm{D}_{6}\right): \delta=1.69(\mathrm{~s}, J=6.5 \mathrm{~Hz}, 3 \mathrm{H}), 5.87(\mathrm{q}, J=6.5 \mathrm{~Hz}, 1 \mathrm{H})$, 6.98-7.06 (m, 3H), 7.41-7.43 (m, 2H) ppm. ${ }^{13} \mathrm{C}$ NMR $\left(100 \mathrm{MHz}, \mathrm{C}_{6} \mathrm{D}_{6}\right): \delta=18.0$, 126.4, 127.2, 128.1 (determined by HMBC), 128.4, 128.5, 140.5 ppm. 
For (E)-(1-bromoprop-1-en-1-yl)benzene $(E-5 d)$

${ }^{1} \mathrm{H}$ NMR $\left(400 \mathrm{MHz}, \mathrm{C}_{6} \mathrm{D}_{6}\right): \delta=1.26(\mathrm{~d}, J=6.5 \mathrm{~Hz}, 3 \mathrm{H}), 6.06(\mathrm{q}, J=6.5 \mathrm{~Hz}, 1 \mathrm{H})$, 7.24-7.26 $(\mathrm{m}, 2 \mathrm{H}) \mathrm{ppm}$. (Signal overlapping with those of the other double bond isomer thwarted the assignment of other resonances.) ${ }^{13} \mathrm{C} \mathrm{NMR}\left(100 \mathrm{MHz}, \mathrm{C}_{6} \mathrm{D}_{6}\right)$ : $16.2,121.1,127.2,129.1,139.0 \mathrm{ppm}$. (Signal overlapping with those of the solvent thwarted the assignment of other resonances.) 


\subsubsection{4-(1-Bromovinyl)benzoic acid (5f)}<smiles></smiles>

Prepared from 4-ethynylbenzoic acid (4f, $25 \mathrm{mg}, 0.17 \mathrm{~mol}$ ) and surrogate 3 (60 $\mathrm{mg}$, $0.23 \mathrm{mmol}, 1.3$ equiv) according to Method A, over 24 hours. The residue was purified by flash column chromatography on silica gel using tert-butyl methyl ether as the eluent to afford 4-(1-bromovinyl)benzoic acid (5f, $29 \mathrm{mg}, 75 \%)$ as a brown viscous oil.

$\mathbf{R}_{\mathbf{f}}=0.60$ (tert-butyl methyl ether). IR (ATR): $\tilde{v}=707,772,858,1288,1421.1605$, 1680, $2840 \mathrm{~cm}^{-1}$. HRMS (APCI) for $\mathrm{C}_{9} \mathrm{H}_{6} \mathrm{BrO}_{2}^{-}\left[\mathrm{M}-\mathrm{H}^{-}\right]$: calculated 224.9557, found 224.9555. ${ }^{1} \mathrm{H}$ NMR (500 MHz, $\left.\mathrm{CD}_{2} \mathrm{Cl}_{2}\right): \delta=5.94(\mathrm{~d}, J=2.5 \mathrm{~Hz}, 1 \mathrm{H}), 6.29(\mathrm{~d}, J=2.5$ $\mathrm{Hz}, 1 \mathrm{H}), 7.72(\mathrm{~d}, J=8.3 \mathrm{~Hz}, 2 \mathrm{H}), 8.09(\mathrm{~d}, J=8.3 \mathrm{~Hz}, 2 \mathrm{H}) \mathrm{ppm} .{ }^{13} \mathrm{C}$ NMR $(125 \mathrm{MHz}$, $\left.\mathrm{CD}_{2} \mathrm{Cl}_{2}\right): \delta=120.5,127.8,129.8,129.9,130.5,143.7,170.6 \mathrm{ppm}$. 


\subsection{Substrate scope with activated acceptors}<smiles>[R]C#CC([R2])=O</smiles>
6a-k
3 (1.5 equiv)
7a-k<smiles>O=C(/C=C(\Br)c1ccccc1)c1ccccc1</smiles><smiles>O=C(O)/C=C(\Br)c1ccccc1</smiles><smiles>[X]c1ccc(/C(Br)=C/C(N)=O)cc1</smiles>

7a: $86 \%, Z: E>99: 17 b: 79 \%, Z: E=79: 217 c(X=H): 84 \%, Z: E=94: 6$<smiles>[Z]c1cccc2ccc(OC)cc12</smiles>

7d $(X=C l): 56 \%, Z: E=84: 16$

7e $\left(X=C F_{3}\right): 91 \%, Z: E=89: 11$

$7 f(X=M e): 87 \%, Z: E=63: 37$<smiles>CC/C(Br)=C/C(=O)N(C)C</smiles>

$7 g: 56 \%, Z: E=75: 25$<smiles>CN(C)C(=O)C=C(Br)C1CCCCC1</smiles><smiles>CCOC(=O)C=C(Br)C1CCCCC1</smiles>

7h: $38 \%, Z: E=77: 23$

7i: $47 \%, Z: E>99: 1$

$7 \mathbf{j}: 43 \%, E: Z=53: 47$<smiles>CC(=O)/C=C(\Br)C(C)=Cc1ccccc1</smiles>

7k: $51 \%, Z: E>99: 1$<smiles>C=C=C(C)C(=O)O[13C](=O)[18OH]</smiles>

8

9: $22 \%, E: Z=91: 9$

Scheme S6. Transfer hydrobromination of activated alkynes 


\subsubsection{3-Bromo-1,3-diphenylprop-2-en-1-one (Z:E > 99:1) (7a)}<smiles>O=C(/C=C(\Br)c1ccccc1)c1ccccc1</smiles>

\section{Z-7a}

$\mathrm{C}_{15} \mathrm{H}_{11} \mathrm{BrO}$

$286.00 \mathrm{~g} / \mathrm{mol}$

Prepared from 1,3-diphenylprop-2-yn-1-one (6a, $26 \mathrm{mg}, 0.13 \mathrm{mmol})$ and surrogate 3 (60 mg, $0.22 \mathrm{mmol}, 1.5$ equiv) according to Method B. The residue was purified by flash column chromatography on silica gel using firstly cyclohexane:tert-butyl methyl ether $=50: 1$ (to remove the solvent and biphenyl) and then cyclohexane:tert-butyl methyl ether $=1: 1$ as the eluent to afford 3-bromo-1,3-diphenylprop-2-en-1-one $(Z: E$ $>99: 1)(7 a, 31 \mathrm{mg}, 86 \%)$ as a viscous yellow oil.

The $Z$ configuration was determined by NOESY measurements.

$\mathbf{R}_{\mathbf{f}}=0.70$ (cyclohexane:tert-butyl methyl ether = 10:1). IR $($ ATR): $\tilde{v}=693,757,1013$, 1226, 1446, 1596, 1667, $3058 \mathrm{~cm}^{-1}$. HRMS (APCl) for $\mathrm{C}_{15} \mathrm{H}_{12} \mathrm{BrO}^{+}\left[\mathrm{M}+\mathrm{H}^{+}\right]$: calculated 287.0066, found 287.0057. ${ }^{1} \mathrm{H}$ NMR (500 MHz, $\left.\mathrm{CD}_{2} \mathrm{Cl}_{2}\right): \delta=7.44-7.47(\mathrm{~m}, 3 \mathrm{H})$, 7.50-7.538 (m, 2H), $7.543(\mathrm{~s}, 1 \mathrm{H}), 7.62(\mathrm{tt}, J=7.4,1.9 \mathrm{~Hz}, 1 \mathrm{H}) 7.72-7.75(\mathrm{~m}, 2 \mathrm{H})$, $8.00(\mathrm{~d}, J=8.4 \mathrm{~Hz}, 2 \mathrm{H}) \mathrm{ppm} .{ }^{13} \mathrm{C}$ NMR $\left(125 \mathrm{MHz}, \mathrm{CD}_{2} \mathrm{Cl}_{2}\right): \delta=126.4,128.4,129.0$, $129.10,129.12,129.18,130.7,133.8,137.7,139.6,190.7$ ppm.

1,3-Diphenylprop-2-yn-1-one (6a) was prepared according to a reported procedure. ${ }^{\text {S2 }}$ 


\subsubsection{3-Bromo-3-phenylacrylic acid $(Z: E=79: 21)(7 b)$}<smiles>O=C(O)/C=C(\Br)c1ccccc1</smiles>

Z-7b

$\mathrm{C}_{9} \mathrm{H}_{7} \mathrm{BrO}_{2}$

$225.96 \mathrm{~g} / \mathrm{mol}$

Prepared from 3-phenylpropiolic acid $(6 \mathrm{~b}, 19 \mathrm{mg}, 0.13 \mathrm{mmol})$ and surrogate 3 (50 $\mathrm{mg}, 0.19 \mathrm{mmol}, 1.4$ equiv) according to Method B. The residue was purified by flash column chromatography on silica gel using firstly cyclohexane:tert-butyl methyl ether $=50: 1$ (to remove the solvent and biphenyl) and then tert-butyl methyl ether as the eluent to afford 3-bromo-3-phenylacrylic acid $(Z: E=79: 21)(7 b, 22 \mathrm{mg}, 79 \%)$ as a yellow viscous oil.

The $Z$ and $E$ configurations were determined by NOESY measurements.

$\mathbf{R}_{\mathbf{f}}=0.30$ (tert-butyl methyl ether). IR (ATR): $\tilde{v}=691,766,1203,1603,1692,2920$, $2989 \mathrm{~cm}^{-1}$. HRMS (APCl) for $\mathrm{C}_{9} \mathrm{H}_{8} \mathrm{BrO}_{2}^{+}\left[\mathrm{M}+\mathrm{H}^{+}\right]$: calculated 226.9702 found 226.9688.

For (Z)-3-bromo-3-phenylacrylic acid (Z-7b):

${ }^{1} \mathrm{H}$ NMR $\left(500 \mathrm{MHz}, \mathrm{CD}_{2} \mathrm{Cl}_{2}\right): \delta=6.79(\mathrm{~s}, 1 \mathrm{H}), 7.40-7.46(\mathrm{~m}, 3 \mathrm{H}), 7.67(\mathrm{~d}, J=8.1 \mathrm{~Hz}$, $2 \mathrm{H})$ ppm. ${ }^{13} \mathrm{C}$ NMR $\left(125 \mathrm{MHz}, \mathrm{CD}_{2} \mathrm{Cl}_{2}\right): \delta=119.5,128.1,128.4,129.0,131.2,140.5$, 167.4 ppm.

For (E)-3-bromo-3-phenylacrylic acid (E-7b):

${ }^{1} \mathrm{H}$ NMR $\left(500 \mathrm{MHz}, \mathrm{CD}_{2} \mathrm{Cl}_{2}\right): \delta=6.65(\mathrm{~s}, 1 \mathrm{H}), 7.40-7.46(\mathrm{~m}, 5 \mathrm{H}) \mathrm{ppm} .{ }^{13} \mathrm{C}$ NMR $(125$ $\mathrm{MHz}, \mathrm{CD}_{2} \mathrm{Cl}_{2}$ ): $\delta=123.3,127.9,128.6,128.7,130.3,139.5$ ppm. (The remaining signal belonging to $\mathrm{COOH}$ could not be identified due to the low quantity of material.) 


\subsubsection{3-Bromo-N,N-dimethyl-3-phenylacrylamide $(Z: E=94: 6)(7 \mathrm{c})$}<smiles>CNC(=O)/C=C(\Br)c1ccccc1</smiles>

Z-7c

$\mathrm{C}_{11} \mathrm{H}_{12} \mathrm{BrNO}$

$253.01 \mathrm{~g} / \mathrm{mol}$<smiles>CN(C)C(=O)/C=C(\Br)c1ccccc1</smiles>

E-7c

$\mathrm{C}_{11} \mathrm{H}_{12} \mathrm{BrNO}$

$253.01 \mathrm{~g} / \mathrm{mol}$

Prepared from N,N-dimethyl-3-phenylpropiolamide (6c, $26 \mathrm{mg}, 0.15 \mathrm{mmol}$ ) and surrogate 3 (60 mg, $0.22 \mathrm{mmol}, 1.5$ equiv) according to Method B. The residue was purified by flash column chromatography on silica gel using firstly cyclohexane:tertbutyl methyl ether $=50: 1$ (to remove the solvent and biphenyl) and then cyclohexane:tert-butyl methyl ether $=1: 1$ as the eluent to afford (Z)-3-bromo- $N, N$ dimethyl-3-phenylacrylamide (Z-7c, $30 \mathrm{mg}, 79 \%)$ as a viscous yellow oil and (E)-3bromo- $N, N$-dimethyl-3-phenylacrylamide $(E-7 c, 6 \mathrm{mg})$ as a yellow viscous oil which contains $74 \%$ of an inseparable unreacted $N, N$-dimethyl-3-phenylpropiolamide (6c). This corresponds to a yield of $5 \%$ of $E-7 c$.

The $Z$ and $E$ configurations were determined by NOESY measurements.

For (Z)-3-iodo-N,N-dimethyl-3-phenylacrylamide (Z-7c):

$\mathbf{R}_{\mathbf{f}}=0.30$ (tert-butyl methyl ether). IR (ATR): $\tilde{v}=693,759,1165,1394,1631,2925$ $\mathrm{cm}^{-1}$. HRMS (APCl) for $\mathrm{C}_{11} \mathrm{H}_{12} \mathrm{BrNO}^{+}\left[\mathrm{M}^{+}\right]$: calculated 253.0102, found 253.0096. ${ }^{1} \mathrm{H}$ NMR (500 MHz, $\left.\mathrm{C}_{6} \mathrm{D}_{6}\right): \delta=2.40(\mathrm{~s}, 3 \mathrm{H}), 2.71(\mathrm{~s}, 3 \mathrm{H}), 6.47(\mathrm{~s}, 1 \mathrm{H}), 6.97-7.00$ (m, $3 \mathrm{H}), 7.35-7.39(\mathrm{~m}, 2 \mathrm{H}) \mathrm{ppm} .{ }^{13} \mathrm{C}$ NMR $\left(125 \mathrm{MHz}, \mathrm{C}_{6} \mathrm{D}_{6}\right): \delta=33.9,36.6,125.8,127.9$ (determined with HMBC), 128.4 (determined with HMBC), 128.6, 129.5, 138.8, 165.7 ppm.

For $(E)-3-i o d o-N, N$-dimethyl-3-phenylacrylamide $(\boldsymbol{E}-7 \mathbf{c})$ :

$\mathbf{R}_{\mathbf{f}}=0.50$ (tert-butyl methyl ether). IR (ATR): $\tilde{v}=762,1133,1489,1618,2923 \mathrm{~cm}^{-1}$. HRMS (APCl) for $\mathrm{C}_{11} \mathrm{H}_{12} \mathrm{BrNO}^{+}\left[\mathrm{M}^{+}\right]$: calculated 253.0102, found 253.0096. ${ }^{1} \mathrm{H}$ NMR $\left(500 \mathrm{MHz}, \mathrm{C}_{6} \mathrm{D}_{6}\right): \delta=1.97(\mathrm{~s}, 3 \mathrm{H}), 2.38(\mathrm{~s}, 3 \mathrm{H}), 6.48(\mathrm{~s}, 1 \mathrm{H}), 7.55$ (d, J = $\left.7.8 \mathrm{~Hz}, 2 \mathrm{H}\right)$ ppm. (Signal overlapping with those of the unreacted starting material thwarted the assignment of other resonances.) ${ }^{13} \mathrm{C}$ NMR (125 MHz, $\mathrm{C}_{6} \mathrm{D}_{6}$ ): Signals could not be identified due to the low quantity of material. 
N,N-Dimethyl-3-phenylpropiolamide (6c) was prepared according to a reported procedure. $^{\mathrm{S} 3}$ 


\subsubsection{3-Bromo-3-(4-chlorophenyl)-N,N-dimethylacrylamide (Z:E = 84:16) (7d)}<smiles>CNC(=O)/C=C(\Br)c1ccc(Cl)cc1</smiles>

Z-7d

$\mathrm{C}_{11} \mathrm{H}_{11} \mathrm{BrCINO}$

$286.97 \mathrm{~g} / \mathrm{mol}$<smiles>CNC(=O)/C=C(\Br)c1ccc(Cl)cc1</smiles>

E-7d

$\mathrm{C}_{11} \mathrm{H}_{11} \mathrm{BrCINO}$

$286.97 \mathrm{~g} / \mathrm{mol}$

Prepared from 3-(4-chlorophenyl)-N,N-dimethylpropiolamide (6d, $31 \mathrm{mg}, 0.15 \mathrm{mmol}$ ) and surrogate 3 (60 mg, $0.22 \mathrm{mmol}, 1.5$ equiv) according to Method B. The residue was purified by flash column chromatography on silica gel using firstly cyclohexane:tert-butyl methyl ether $=50: 1$ (to remove the solvent and biphenyl) and tert-butyl methyl ether as the eluent to afford (Z)- 3-bromo-3-(4-chlorophenyl)- $N, N$ dimethylacrylamide (Z-7d, $21 \mathrm{mg}, 49 \%)$ as a viscous yellow oil and $(E)$ - 3-bromo-3(4-chlorophenyl)- $N, N$-dimethylacrylamide $(\boldsymbol{E}-7 \mathbf{d}, 5 \mathrm{mg})$ as a yellow viscous oil which contains $52 \%$ of an inseparable unreacted 3-(4-chlorophenyl)- $N, N$ dimethylpropiolamide (6d). This corresponds to a yield of $7 \%$ of $\mathbf{E - 7 d}$.

The $Z$ and $E$ configurations were determined by NOESY measurements.

For (Z)-3-bromo-3-(4-chlorophenyl)-N,N-dimethylacrylamide (Z-7d):

$\mathbf{R}_{\mathbf{f}}=0.50$ (tert-butyl methyl ether). IR (ATR): $\tilde{v}=823,1091,1396,1487,1633,2926$ $\mathrm{cm}^{-1}$. HRMS (APCl) for $\mathrm{C}_{11} \mathrm{H}_{11} \mathrm{BrCINO}^{+}\left[\mathrm{M}^{+}\right]$: calculated 286.9785, found 286.9780. ${ }^{1} \mathrm{H}$ NMR (400 MHz, $\left.\mathrm{C}_{6} \mathrm{D}_{6}\right): \delta=2.35(\mathrm{~s}, 3 \mathrm{H}), 2.73(\mathrm{~s}, 3 \mathrm{H}), 6.31$ (s, $\left.1 \mathrm{H}\right), 6.94$ (d, J = 8.0 $\mathrm{Hz}, 2 \mathrm{H}), 7.03$ (d, J = 8.0 Hz, 2H) ppm. ${ }^{13} \mathrm{C}$ NMR $\left(100 \mathrm{MHz}, \mathrm{C}_{6} \mathrm{D}_{6}\right): \delta=33.9,36.5$, $126.3,126.4,128.7,129.0,135.5,137.1,165.4$ ppm.

For (E)- 3-bromo-3-(4-chlorophenyl)-N,N-dimethylacrylamide (E-7d):

$\mathbf{R}_{\mathbf{f}}=0.30$ (tert-butyl methyl ether). IR (ATR): $\tilde{v}=830,1014,1092,1395,1489,1632$, $2926 \mathrm{~cm}^{-1}$. HRMS (APCl) for $\mathrm{C}_{11} \mathrm{H}_{11} \mathrm{BrClNO}^{+}\left[\mathrm{M}^{+}\right]$: calculated 286.9785, found 286.9780. ${ }^{1} \mathrm{H}$ NMR (400 MHz, $\left.\mathrm{C}_{6} \mathrm{D}_{6}\right): \delta=2.05(\mathrm{~s}, 3 \mathrm{H}), 2.46(\mathrm{~s}, 3 \mathrm{H}), 6.50(\mathrm{~s}, 1 \mathrm{H}), 6.93$ $(\mathrm{d}, J=8.0 \mathrm{~Hz}, 2 \mathrm{H}), 7.37(\mathrm{~d}, J=8.0 \mathrm{~Hz}, 2 \mathrm{H}) \mathrm{ppm} .{ }^{13} \mathrm{C} \mathrm{NMR}\left(100 \mathrm{MHz}, \mathrm{C}_{6} \mathrm{D}_{6}\right): \delta=$ $33.8,36.3,126.8,127.4,128.5,128.6,135.6,136.9,164.7$ ppm.

3-(4-Chlorophenyl)-N,N-dimethylpropiolamide (6d) was prepared according to a reported procedure. ${ }^{\mathrm{S} 3}$ 


\subsubsection{3-Bromo-N,N-dimethyl-3-(4-(trifluoromethyl)phenyl)acrylamide (Z:E =} 89:11) (7e)<smiles>CNC(=O)/C=C(\Br)c1ccc(C(F)(F)F)cc1</smiles>

Z-7e

$\mathrm{C}_{12} \mathrm{H}_{11} \mathrm{BrF}_{3} \mathrm{NO}$

$321.00 \mathrm{~g} / \mathrm{mol}$<smiles>CNC(=O)/C=C(/Br)c1ccc(C(F)(F)F)cc1</smiles>

E-7e

$\mathrm{C}_{12} \mathrm{H}_{11} \mathrm{BrF}_{3} \mathrm{NO}$

$321.00 \mathrm{~g} / \mathrm{mol}$

Prepared from N,N-dimethyl-3-(4-(trifluoromethyl)phenyl)propiolamide (6e, $36 \mathrm{mg}$, $0.15 \mathrm{mmol}$ ) and surrogate 3 (60 $\mathrm{mg}, 0.22 \mathrm{mmol}, 1.5$ equiv) according to Method $\mathbf{B}$. The residue was purified by flash column chromatography on silica gel using firstly cyclohexane:tert-butyl methyl ether $=50: 1$ (to remove the solvent and biphenyl) and tert-butyl methyl ether as the eluent to afford (Z)-3-bromo-N,N-dimethyl-3-(4(trifluoromethyl)phenyl)acrylamide (Z-7e, $39 \mathrm{mg}, 81 \%$ ) as a viscous yellow oil and (E)-3-bromo-N,N-dimethyl-3-(4-(trifluoromethyl)phenyl)acrylamide (E-7e, $6 \mathrm{mg}$ ) as a yellow viscous oil which contains $17 \%$ of an inseparable unreacted $N, N$-dimethyl-3(4-(trifluoromethyl)phenyl)propiolamide (6e). This corresponds to a yield of $10 \%$ of $E$ 7 e.

The $Z$ and $E$ configurations were determined by NOESY measurements.

For (Z)-3-bromo-N,N-dimethyl-3-(4-(trifluoromethyl)phenyl)acrylamide (Z-7e):

$\mathbf{R}_{\mathbf{f}}=0.40$ (tert-butyl methyl ether). IR (ATR): $\tilde{v}=832,1068,1118,1166,1324,1399$, $1637 \mathrm{~cm}^{-1}$. HRMS (APCl) for $\mathrm{C}_{12} \mathrm{H}_{12} \mathrm{BrF}_{3} \mathrm{NO}^{+}\left[\mathrm{M}+\mathrm{H}^{+}\right]$: calculated 322.0049 , found 322.0040. ${ }^{1} \mathrm{H}$ NMR (400 MHz, $\left.\mathrm{C}_{6} \mathrm{D}_{6}\right): \delta=2.48(\mathrm{~s}, 3 \mathrm{H}), 2.82(\mathrm{~s}, 3 \mathrm{H}), 6.49(\mathrm{~s}, 1 \mathrm{H})$, 7.23-7.30 (m, 4H) ppm. ${ }^{13} \mathrm{C}$ NMR $\left(100 \mathrm{MHz}, \mathrm{C}_{6} \mathrm{D}_{6}\right): \delta=33.8,36.4,123.2,125.1$ (q, J $=4.2 \mathrm{~Hz}), 125.9,127.6,128.2,131.0$ (q, $J=33.3 \mathrm{~Hz}), 141.8,165.2 \mathrm{ppm} .{ }^{19} \mathrm{~F}$ NMR $\left(659 \mathrm{MHz}, \mathrm{C}_{6} \mathrm{D}_{6}\right): \delta=-62.5 \mathrm{ppm}$.

For (E)-3-bromo-N,N-dimethyl-3-(4-(trifluoromethyl)phenyl)acrylamide $(\boldsymbol{E}-7 \mathbf{e})$ :

$\mathbf{R}_{\mathbf{f}}=0.60$ (tert-butyl methyl ether). IR (ATR): $\tilde{v}=811,1066,1130,1323,1639,2277$ $\mathrm{cm}^{-1}$. HRMS (APCl) for $\mathrm{C}_{12} \mathrm{H}_{12} \mathrm{BrF}_{3} \mathrm{NO}^{+}\left[\mathrm{M}+\mathrm{H}^{+}\right]$: calculated 322.0049, found 322.0040. ${ }^{1} \mathrm{H}$ NMR (400 MHz, $\left.\mathrm{C}_{6} \mathrm{D}_{6}\right): \delta=1.92(\mathrm{~s}, 3 \mathrm{H}), 2.34(\mathrm{~s}, 3 \mathrm{H}), 6.42(\mathrm{~s}, 1 \mathrm{H}), 7.13$ $(\mathrm{d}, J=8.4 \mathrm{~Hz}, 2 \mathrm{H}), 7.36(\mathrm{~d}, J=8.4 \mathrm{~Hz}, 2 \mathrm{H}) \mathrm{ppm} .{ }^{13} \mathrm{C}$ NMR $\left(100 \mathrm{MHz}, \mathrm{C}_{6} \mathrm{D}_{6}\right): \delta=$ 
33.8, 36.2, 125.3 (q, $J=4.2 \mathrm{~Hz}), 126.5,129.3,129.5,131.2$ (q, $J=33.3 \mathrm{~Hz}), 132.6$, 164.3 ppm. ${ }^{19} \mathrm{~F}$ NMR $\left(659 \mathrm{MHz}, \mathrm{C}_{6} \mathrm{D}_{6}\right): \delta=-62.6 \mathrm{ppm}$.

$\mathrm{N}, \mathrm{N}$-Dimethyl-3-(4-(trifluoromethyl)phenyl)propiolamide (6e) was prepared according to a reported procedure. ${ }^{\mathrm{S3}}$ 


\subsubsection{3-Bromo-N,N-dimethyl-3-(p-tolyl)acrylamide (7f)}<smiles>Cc1ccc(/C(Br)=C/C(=O)N(C)C)cc1</smiles>

Z-7f

$\mathrm{C}_{12} \mathrm{H}_{14} \mathrm{BrNO}$

$267.06 \mathrm{~g} / \mathrm{mol}$<smiles>Cc1ccc(/C(Br)=C\C(=O)N(C)C)cc1</smiles>

E-7f

$\mathrm{C}_{12} \mathrm{H}_{14} \mathrm{BrNO}$

$267.06 \mathrm{~g} / \mathrm{mol}$

On $1.0 \mathrm{mmol}$ scale:

According to a modified procedure from method $\mathrm{B}$, in a glovebox, a pressure tube is charged with a solution of N,N-dimethyl-3-(p-tolyl)propiolamide (6f, $200 \mathrm{mg}, 1.07$ $\mathrm{mmol}$ ) and 1 surrogate 3 (400 mg, $153 \mathrm{mmol}, 1.5$ equiv) in chlorobenzene $(2.0 \mathrm{~mL})$, and a solution of $\mathrm{Tf}_{2} \mathrm{NH}(60 \mathrm{mg}, 0.21 \mathrm{mmol}, 20 \mathrm{~mol} \%)$ in chlorobenzene $(1.0 \mathrm{~mL})$ is added. The tube is sealed and removed from the glove box and stirred in a preheated $160{ }^{\circ} \mathrm{C}$ bath for 5 days. $\mathrm{NH}_{4} \mathrm{Cl}$ (sat., aq., $10 \mathrm{~mL}$ ) was added and the mixture was extracted with tert-butyl methyl ether $(3 \times 15 \mathrm{~mL})$. The combined organic phases were washed with brine $(30 \mathrm{~mL})$, dried over $\mathrm{MgSO}_{4}$ and concentrated under reduced pressure. The residue was purified by flash column chromatography on silica gel using firstly cyclohexane:tert-butyl methyl ether $=50: 1$ (to remove the solvent and biphenyl) and then cyclohexane:tert-butyl methyl ether $=1: 1$ as the eluent to afford (Z)-3-bromo- $N, N$-dimethyl-3-(p-tolyl)acrylamide (Z-7f, $137 \mathrm{mg}, 48 \%$ ) as a viscous yellow oil and $(E)$-33-bromo- $N, N$-dimethyl-3-( $p$-tolyl)acrylamide (E-7f, 20 $\mathrm{mg}, 7 \%)$ as a yellow viscous oil. The unreacted $N, N$-dimethyl-3-( $p$-tolyl)propiolamide (6c, $70 \mathrm{mg}, 36 \%)$ was recovered as a brown solid.

On $0.15 \mathrm{mmol}$ scale:

Prepared from N,N-dimethyl-3-(p-tolyl)propiolamide (6f, $28 \mathrm{mg}, 0.15 \mathrm{mmol})$ and surrogate 3 (60 mg, $0.22 \mathrm{mmol}, 1.5$ equiv) according to Method B. The residue was purified by flash column chromatography on silica gel using firstly cyclohexane:tertbutyl methyl ether $=50: 1$ (to remove the solvent and biphenyl) and tert-butyl methyl ether as the eluent to afford (Z)- 3-bromo- $N, N$-dimethyl-3-( $p$-tolyl)acrylamide (Z-7f, $22 \mathrm{mg}, 55 \%)$ as a colorless oil and (E)-3-bromo- $N, N$-dimethyl-3-( $p$-tolyl)acrylamide (E-7f, $13 \mathrm{mg}, 32 \%)$ as a colorless oil.

The $Z$ and $E$ configurations were determined by NOESY measurements. 
For (Z)-3-bromo-N,N-dimethyl-3-(p-tolyl)acrylamide (Z-7f):

$\mathbf{R}_{\mathbf{f}}=0.50$ (tert-butyl methyl ether). IR (ATR): $\tilde{v}=812,1162,1391,1626,2920 \mathrm{~cm}^{-1}$. HRMS (APCl) for $\mathrm{C}_{12} \mathrm{H}_{15} \mathrm{BrNO}^{+}\left[\mathrm{M}+\mathrm{H}^{+}\right]$: calculated 268.0332, found 268.0325. ${ }^{1} \mathrm{H}$ NMR $\left(400 \mathrm{MHz}, \mathrm{C}_{6} \mathrm{D}_{6}\right): \delta=2.01(\mathrm{~s}, 3 \mathrm{H}), 2.41(\mathrm{~s}, 3 \mathrm{H}), 2.72(\mathrm{~s}, 3 \mathrm{H}), 6.50(\mathrm{~s}, 1 \mathrm{H}), 6.84$ $(\mathrm{d}, J=7.7 \mathrm{~Hz}, 2 \mathrm{H}), 7.34$ (d, $J=7.7 \mathrm{~Hz}, 2 \mathrm{H}) \mathrm{ppm} .{ }^{13} \mathrm{C} \mathrm{NMR}\left(100 \mathrm{MHz}, \mathrm{C}_{6} \mathrm{D}_{6}\right): \delta=$ 21.0, 33.9, 36.6, 125.0, 128.0 (determined by HMQC), 128.2 (determined by HMBC), $129.3,136.1,139.6,165.8 \mathrm{ppm}$.

For (E)-3-bromo- $N, N$-dimethyl-3-( $p$-tolyl)acrylamide $(E-7 f)$ :

$\mathbf{R}_{\mathbf{f}}=0.30$ (tert-butyl methyl ether). IR (ATR): $\tilde{v}=812,1162,1391,1626,2920 \mathrm{~cm}^{-1}$. HRMS (APCl) for $\mathrm{C}_{12} \mathrm{H}_{15} \mathrm{BrNO}^{+}\left[\mathrm{M}+\mathrm{H}^{+}\right]$: 268.0332, found 268.0325. ${ }^{1} \mathrm{H}$ NMR (400 $\left.\mathrm{MHz}, \mathrm{C}_{6} \mathrm{D}_{6}\right): \delta=1.91(\mathrm{~s}, 3 \mathrm{H}), 2.20(\mathrm{~s}, 3 \mathrm{H}), 2.42(\mathrm{~s}, 3 \mathrm{H}), 6.47(\mathrm{~s}, 1 \mathrm{H}), 6.77(\mathrm{~d}, J=7.7$ $\mathrm{Hz}, 2 \mathrm{H}), 7.50$ (d, $J=7.7 \mathrm{~Hz}, 2 \mathrm{H}) \mathrm{ppm} .{ }^{13} \mathrm{C}$ NMR $\left(100 \mathrm{MHz}, \mathrm{C}_{6} \mathrm{D}_{6}\right): \delta=21.0,33.9$, $36.4,127.6,128.9,129.0,129.5,132.5,135.8,165.3 \mathrm{ppm}$.

$\mathrm{N}, \mathrm{N}$-Dimethyl-3-( $p$-tolyl)propiolamide (6f) was prepared according to a reported procedure. $^{\text {S3 }}$ 
3.3.7 3-Bromo-3-(6-methoxynaphthalen-2-yl)-N,N-dimethylacrylamide $(Z: E=$ 75:25) (7g)
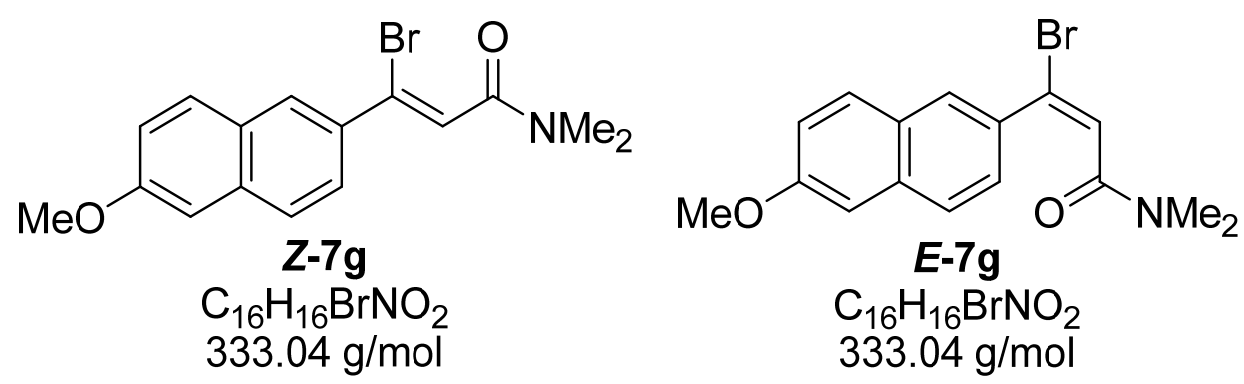

Prepared from 3-(6-methoxynaphthalen-2-yl)-N,N-dimethylpropiolamide $(\mathbf{6 g}, 32 \mathrm{mg}$, $0.15 \mathrm{mmol}$ ) and surrogate 3 (60 mg, $0.22 \mathrm{mmol}, 1.5$ equiv) according to Method $\mathbf{B}$. The residue was purified by flash column chromatography on silica gel using firstly cyclohexane:tert-butyl methyl ether $=50: 1$ (to remove the solvent and biphenyl) and tert-butyl methyl ether as the eluent to afford (Z)-3-bromo-3-(6-methoxynaphthalen-2yl)-N,N-dimethylacrylamide (Z-7g, $21 \mathrm{mg}, 42 \%)$ as a colorless oil and (E)-3-bromo-3(6-methoxynaphthalen-2-yl)- $N, N$-dimethylacrylamide $(E-7 g, 8 \mathrm{mg})$ as a colorless oil which contains $18 \%$ of an inseparable unreacted 3-(6-methoxynaphthalen-2-yl)-N,Ndimethylpropiolamide $(\mathbf{6 g})$. This corresponds to a yield of $14 \%$ of $\mathbf{E - 7 g}$.

The $Z$ and $E$ configurations were determined by NOESY measurements.

For (Z)-3-bromo-3-(6-methoxynaphthalen-2-yl)-N,N-dimethylacrylamide (Z-7g):

$\mathbf{R}_{\mathbf{f}}=0.20$ (tert-butyl methyl ether). IR (ATR): $\tilde{v}=854,1029,1167,1267,1392,1630$, $2932 \mathrm{~cm}^{-1}$. HRMS (APCl) for $\mathrm{C}_{16} \mathrm{H}_{17} \mathrm{BrNO}_{2}^{+}\left[\mathrm{M}+\mathrm{H}^{+}\right]$: calculated 334.0437, found 334.0429. ${ }^{1} \mathrm{H}$ NMR (400 MHz, $\left.\mathrm{C}_{6} \mathrm{D}_{6}\right): \delta=2.58(\mathrm{~s}, 3 \mathrm{H}), 2.87(\mathrm{~s}, 3 \mathrm{H}), 3.48(\mathrm{~s}, 3 \mathrm{H}), 6.71$ (s, 1H), 6.95-6.97 (m, 1H), $7.23(\mathrm{~d}, J=2.5 \mathrm{~Hz}, 1 \mathrm{H}), 7.52-7.56(\mathrm{~m}, 2 \mathrm{H}), 7.62$ (dd, $J=$ 8.0, $2.5 \mathrm{~Hz}, 1 \mathrm{H}), 8.00-8.02(\mathrm{~m}, 1 \mathrm{H}) \mathrm{ppm} .{ }^{13} \mathrm{C}$ NMR $\left(100 \mathrm{MHz}, \mathrm{C}_{6} \mathrm{D}_{6}\right): \delta=33.9,36.7$, 54.8, 105.9, 119.9, 125.3, 125.4, 127.2, 127.4 (determined by HMBC), 128.2, 128.8 (determined by HMBC), 130.5, 133.8, 135.5, 159.1, 165.9 ppm.

For (E)-3-bromo-3-(6-methoxynaphthalen-2-yl)-N,N-dimethylacrylamide (E-7g):

$\mathbf{R}_{\mathbf{f}}=0.40$ (tert-butyl methyl ether). IR (ATR): $\tilde{v}=811,1031,1163,1328,1635,2277$, $2934 \mathrm{~cm}^{-1}$. HRMS (APCl) for $\mathrm{C}_{16} \mathrm{H}_{17} \mathrm{BrNO}_{2}\left[\mathrm{M}+\mathrm{H}^{+}\right]$: calculated 334.0437, found 334.0429. ${ }^{1} \mathrm{H}$ NMR (400 MHz, $\left.\mathrm{C}_{6} \mathrm{D}_{6}\right): \delta=2.00(\mathrm{~s}, 3 \mathrm{H}), 2.38(\mathrm{~s}, 3 \mathrm{H}), 3.28(\mathrm{~s}, 3 \mathrm{H}), 6.57$ (s, 1H), $6.76(\mathrm{~d}, J=2.5 \mathrm{~Hz}, 1 \mathrm{H}), 7.05(\mathrm{dd}, J=8.5,2.5 \mathrm{~Hz}, 1 \mathrm{H}), 7.34(\mathrm{~d}, J=8.5 \mathrm{~Hz}$, $1 \mathrm{H}), 7.41(\mathrm{~d}, J=8.8 \mathrm{~Hz}, 1 \mathrm{H}), 7.69(\mathrm{dd}, J=8.8,2.0 \mathrm{~Hz}, 1 \mathrm{H}), 8.06(\mathrm{~d}, J=2.0 \mathrm{~Hz}, 1 \mathrm{H})$ 
ppm. ${ }^{13} \mathrm{C}$ NMR $\left(100 \mathrm{MHz}, \mathrm{C}_{6} \mathrm{D}_{6}\right): \delta=33.9,36.5,54.8,105.9,119.9,126.9,127.9$ (determined by HMQC), 128.6, 128.9, 129.2, 130.6, 133.3, 133.6, 135.4, 159.3, $165.5 \mathrm{ppm}$.

3-(6-Methoxynaphthalen-2-yl)- $N, N$-dimethylpropiolamide $\quad(6 \mathbf{g})$ was prepared according to a reported procedure. ${ }^{\mathrm{S3}}$ 


\subsubsection{3-Bromo-3-cyclopropyl-N,N-dimethylacrylamide $(Z: E=77: 23)(7 \mathrm{~h})$}<smiles>CNC(=O)C=C(Br)C1CC1</smiles>

Z-7h

$\mathrm{C}_{8} \mathrm{H}_{12} \mathrm{BrNO}$ $217.01 \mathrm{~g} / \mathrm{mol}$<smiles>CNC(=O)/C=C(/Br)C1CC1</smiles>

E-7h

$\mathrm{C}_{8} \mathrm{H}_{12} \mathrm{BrNO}$

$217.01 \mathrm{~g} / \mathrm{mol}$

Prepared from 3-cyclopropyl-N,N-dimethylpropiolamide $(6 \mathrm{~h}, 20 \mathrm{mg}, 0.15 \mathrm{mmol}$ ) and surrogate 3 (60 mg, 0.22 mmol, 1.5 equiv) according to Method B. The residue was purified by flash column chromatography on silica gel using firstly cyclohexane:tertbutyl methyl ether $=50: 1$ (to remove the solvent and biphenyl) and then cyclohexane:tert-butyl methyl ether $=1: 1$ as the eluent to afford 3-bromo-3cyclopropyl- $N, N$-dimethylacrylamide $(7 \mathbf{h}, 17 \mathrm{mg})$ as a viscous yellow oil which contains $38 \%$ of an inseparable unreacted 3-cyclopropyl- $N, N$-dimethylpropiolamide (6h). This corresponds to a yield of $38 \%$ of $7 \mathrm{~h}(Z: E=77: 23)$.

The $Z$ and $E$ configurations were determined by NOESY measurements.

$\mathbf{R}_{\mathbf{f}}=0.30$ (tert-butyl methyl ether). IR (ATR): $\tilde{v}=733,1126,1395,1632,2233,2927$ $\mathrm{cm}^{-1}$. HRMS (APCl) for $\mathrm{C}_{8} \mathrm{H}_{13} \mathrm{BrNO}^{+}\left[\mathrm{M}+\mathrm{H}^{+}\right]$: calculated 218.0175, found 218.0172.

For (Z)-3-bromo-3-cyclopropyl-N,N-dimethylacrylamide (Z-7h):

${ }^{1} \mathrm{H}$ NMR (400 MHz, $\left.\mathrm{C}_{6} \mathrm{D}_{6}\right): \delta=0.47-0.51(\mathrm{~m}, 2 \mathrm{H}), 0.90-0.92(\mathrm{~m}, 2 \mathrm{H}), 2.10(\mathrm{~s}, 3 \mathrm{H})$, $2.58(\mathrm{~s}, 3 \mathrm{H}), 3.18-3.25(\mathrm{~m}, 1 \mathrm{H}), 6.42(\mathrm{~s}, 1 \mathrm{H}) \mathrm{ppm} .{ }^{13} \mathrm{C} \mathrm{NMR}\left(100 \mathrm{MHz}, \mathrm{C}_{6} \mathrm{D}_{6}\right): \delta=$ $8.4,16.7,34.7,36.5,124.1,146.0,164.8 \mathrm{ppm}$.

For (E)-3-bromo-3-cyclopropyl-N,N-dimethylacrylamide (E-7h):

${ }^{1} \mathrm{H}$ NMR $\left(400 \mathrm{MHz}, \mathrm{C}_{6} \mathrm{D}_{6}\right): \delta=2.38(\mathrm{~s}, 3 \mathrm{H}), 2.67(\mathrm{~s}, 3 \mathrm{H}), 6.03(\mathrm{~s}, 1 \mathrm{H}) \mathrm{ppm}$. (Signal overlapping with those of the other double bond isomer thwarted the assignment of other resonances.) ${ }^{13} \mathrm{C}$ NMR (100 MHz, $\left.\mathrm{C}_{6} \mathrm{D}_{6}\right)$ : $\delta=7.4,20.8,33.9,36.6,122.7 \mathrm{ppm}$. (The remaining signals could not be identified due to the low quantity of material.)

3-Cyclopropyl-N,N-dimethylpropiolamide (6h) was prepared according to a reported procedure. $^{\text {s3 }}$ 


\subsubsection{3-Bromo-3- cyclohexyl-N,N-dimethylacrylamide (Z:E > 99:1) (7i)}<smiles>CNC(=O)C=C(Br)C1CCCCC1</smiles>

\section{Z-7i}

$\mathrm{C}_{11} \mathrm{H}_{18} \mathrm{BrNO}$

$259.06 \mathrm{~g} / \mathrm{mol}$

Prepared from 3-cyclohexyl-N,N-dimethylpropiolamide (6i, $23 \mathrm{mg}, 0.13 \mathrm{mmol}$ ) and surrogate 3 (50 mg, $0.19 \mathrm{mmol}, 1.5$ equiv) according to Method B. The residue was purified by flash column chromatography on silica gel using firstly cyclohexane:tertbutyl methyl ether $=50: 1$ (to remove the solvent and biphenyl) and then cyclohexane:tert-butyl methyl ether $=1: 1$ as the eluent to afford 3-bromo-3cyclohexyl- $N, N$-dimethylacrylamide $(7 \mathbf{i}, 17 \mathrm{mg}$ ) as a viscous yellow oil which contains $11 \%$ of an inseparable unreacted 3-cyclohexyl- $N, N$-dimethylpropiolamide (6i). This corresponds to a yield of $47 \%$ of $6 \mathbf{i}(Z: E>99: 1)$.

The $Z$ configuration was determined by NOESY measurements.

$\mathbf{R}_{\mathbf{f}}=0.30$ (tert-butyl methyl ether). IR (ATR): $\tilde{v}=804,975,1166,1392,1628,2852$, $2924 \mathrm{~cm}^{-1}$. HRMS (APCl) for $\mathrm{C}_{11} \mathrm{H}_{19} \mathrm{BrNO}^{+}\left[\mathrm{M}+\mathrm{H}^{+}\right]$: calculated 260.0645, found 260.0646. ${ }^{1} \mathrm{H}$ NMR $\left(400 \mathrm{MHz}, \mathrm{CD}_{2} \mathrm{Cl}_{2}\right): \delta=1.18-1.30(\mathrm{~m}, 6 \mathrm{H}), 1.68-1.78(\mathrm{~m}, 2 \mathrm{H})$, 1.82-1.87 (m, 2H), 2.14-2.22 (m, 1H), $2.85(\mathrm{~s}, 3 \mathrm{H}), 2.90(\mathrm{~s}, 3 \mathrm{H}), 6.24(\mathrm{~s}, 1 \mathrm{H}) \mathrm{ppm}$. ${ }^{13} \mathrm{C}$ NMR $\left(100 \mathrm{MHz}, \mathrm{CD}_{2} \mathrm{Cl}_{2}\right): \delta=26.2,26.3,32.4,34.3,37.6,49.0,122.2,128.3$, 167.0 ppm.

3-Cyclohexyl- $N, N$-dimethylpropiolamide (6i) was prepared according to a reported procedure. $^{\text {s3 }}$ 


\subsubsection{Ethyl 3-bromo-3-cyclohexylacrylate $(E: Z=53: 47)(7 \mathrm{j})$}<smiles>CCOC(=O)C=C(Br)C1CCCCC1</smiles>

\section{Z-7j}

$\mathrm{C}_{11} \mathrm{H}_{17} \mathrm{BrO}_{2}$

$260.04 \mathrm{~g} / \mathrm{mol}$

Prepared from ethyl 3-cyclohexylpropiolate $(6 \mathbf{j}, 30 \mathrm{mg}, 0.17 \mathrm{mmol}$ ) and surrogate 3 (60 mg, $0.23 \mathrm{mmol}, 1.2$ equiv) according to Method B. The residue was purified by flash column chromatography on silica gel using firstly cyclohexane:tert-butyl methyl ether $=50: 1$ (to remove the solvent and biphenyl) and then cyclohexane:tert-butyl methyl ether $=30: 1$ as the eluent to afford ethyl 3-bromo-3-cyclohexylacrylate $(\mathbf{7 j}, 33$ $\mathrm{mg}$ ) as a colorless oil which contains $53 \%$ of unreacted ethyl 3-cyclohexylpropiolate (6j). This corresponds to a yield of $43 \%$ of $7 \mathbf{j}(E: Z=53: 47)$.

The $Z$ configuration was determined by NOESY measurements.

$\mathbf{R}_{\mathbf{f}}=0.50$ (cyclohexane:tert-butyl methyl ether = 30:1). IR $($ ATR): $\tilde{v}=811,1181,1246$, 1448, 1709, 2277, $2930 \mathrm{~cm}^{-1}$. HRMS (APCI) for $\mathrm{C}_{11} \mathrm{H}_{18} \mathrm{BrO}_{2}^{+}\left[\mathrm{M}+\mathrm{H}^{+}\right]$: calculated 261.0485, found 261.0488 .

For (E)-3-bromo-3-cyclohexylacrylate $(E-7 \mathbf{j})$ :

${ }^{1} \mathrm{H}$ NMR $\left(400 \mathrm{MHz}, \mathrm{C}_{6} \mathrm{D}_{6}\right): \delta=0.87-0.98(\mathrm{~m}, 2 \mathrm{H}), 0.98(\mathrm{t}, \mathrm{J}=7.9 \mathrm{~Hz}, 3 \mathrm{H}), 1.06-1.17$ $(\mathrm{m}, 1 \mathrm{H}), 1.22-1.34(\mathrm{~m}, 2 \mathrm{H}), 1.44-1.55(\mathrm{~m}, 2 \mathrm{H}), 1.62-1.68(\mathrm{~m}, 2 \mathrm{H}), 4.01$ (q, J = 7.9 $\mathrm{Hz}, 2 \mathrm{H}), 4.08(\mathrm{tt}, J=11.4,3.3 \mathrm{~Hz}, 1 \mathrm{H}), 6.38(\mathrm{~s}, 1 \mathrm{H}) \mathrm{ppm} .{ }^{13} \mathrm{C}$ NMR $\left(100 \mathrm{MHz}, \mathrm{C}_{6} \mathrm{D}_{6}\right)$ : $\delta=14.3,25.9,26.0,32.2,42.9,30.3,157.7,164.2$ ppm.

For (Z)-3-bromo-3-cyclohexylacrylate (Z-7j):

${ }^{1} \mathrm{H}$ NMR $\left(400 \mathrm{MHz}, \mathrm{C}_{6} \mathrm{D}_{6}\right): \delta=0.87(\mathrm{t}, J=7.9 \mathrm{~Hz}, 3 \mathrm{H}), 0.91-1.03(\mathrm{~m}, 2 \mathrm{H}), 1.03-1.24$ (m, 2H), 1.37-1.46 (m, 4H), 1.54-1.71 (m, 2H), $1.97(\mathrm{tt}, J=11.4,3.3 \mathrm{~Hz}, 1 \mathrm{H}), 3.87$ (t, $J=7.9 \mathrm{~Hz}, 2 \mathrm{H}), 6.16(\mathrm{~s}, 1 \mathrm{H}) \mathrm{ppm} .{ }^{13} \mathrm{C}$ NMR $\left(100 \mathrm{MHz}, \mathrm{C}_{6} \mathrm{D}_{6}\right): \delta=14.1,25.8,26.0$, $32.1,50.6,60.3,118.7,147.9,164.1$ ppm. 


\subsubsection{1 (4E)-3-Bromo-N,N,4-trimethyl-5-phenylpenta-2,4-dienamide (Z:E > 99:1)} (7k)<smiles>CC(=CC(=O)N(C)C)C(Br)=Cc1ccccc1</smiles>

$$
\begin{gathered}
\text { Z-7k } \\
\mathrm{C}_{14} \mathrm{H}_{16} \mathrm{BrNO} \\
293.04 \mathrm{~g} / \mathrm{mol}
\end{gathered}
$$

Prepared from (E)-N,N,4-trimethyl-5-phenylpent-4-en-2-ynamide (6k, 27 mg, 0.13 $\mathrm{mmol}$ ) and surrogate 3 (50 $\mathrm{mg}, 0.19 \mathrm{mmol}, 1.5$ equiv) according to Method B. The residue was purified by flash column chromatography on silica gel using firstly cyclohexane:tert-butyl methyl ether $=50: 1$ (to remove the solvent and biphenyl) and then cyclohexane:tert-butyl methyl ether $=30: 1$ as the eluent to afford $(4 E)$-3-bromo$N, N, 4$-trimethyl-5-phenylpenta-2,4-dienamide $(Z: E>99: 1)(7 \mathrm{k}, 19 \mathrm{mg}, 51 \%)$ as a colorless viscous oil.

The $Z$ configuration was determined by NOESY measurements.

$\mathbf{R}_{\mathbf{f}}=0.30$ (tert-butyl methyl ether). IR (ATR): $\tilde{v}=700,749,1162,1395,1639,2923$ $\mathrm{cm}^{-1}$. HRMS (APCI) for $\mathrm{C}_{14} \mathrm{H}_{17} \mathrm{BrNO}^{+}\left[\mathrm{M}+\mathrm{H}^{+}\right]$: calculated 294.0488, found 294.0488. ${ }^{1} \mathrm{H}$ NMR (500 MHz, $\left.\mathrm{C}_{6} \mathrm{D}_{6}\right): \delta=2.04(\mathrm{~d}, J=1.2 \mathrm{~Hz}, 3 \mathrm{H}), 2.91(\mathrm{~s}, 3 \mathrm{H}), 2.97(\mathrm{~s}, 3 \mathrm{H})$, $6.59(\mathrm{~s}, 1 \mathrm{H}), 7.10-7.11(\mathrm{~m}, 1 \mathrm{H}), 7.20-7.25(\mathrm{~m}, 3 \mathrm{H}), 7.29(\mathrm{~d}, \mathrm{~J}=7.1 \mathrm{~Hz}, 2 \mathrm{H}) \mathrm{ppm} .{ }^{13} \mathrm{C}$ NMR $\left(125 \mathrm{MHz}, \mathrm{C}_{6} \mathrm{D}_{6}\right): \delta=16.4,34.3,37.7,124.7,127.8,128.6,129.7,131.9,134.0$, $134.5,137.3,167.0 \mathrm{ppm}$.

(E)-N,N,4-Trimethyl-5-phenylpent-4-en-2-ynamide (6k) was prepared according to a reported procedure. ${ }^{\mathrm{S} 3}$ 


\subsubsection{3-lodo-2-methylbut-2-enoic acid $(E: Z=91: 9)(9)$}<smiles>CC(Br)=C(C)C(=O)O</smiles>

\section{E-7a}

$\mathrm{C}_{5} \mathrm{H}_{7} \mathrm{BrO}_{2}$

$177.96 \mathrm{~g} / \mathrm{mol}$

Prepared from 2-methylbuta-2,3-dienoic acid $(8,20 \mathrm{mg}, 0.20 \mathrm{mmol})$ and surrogate 3 (70 mg, $0.26 \mathrm{mmol}, 1.3$ equiv) according to Method B over 72 hours. The residue was purified by flash column chromatography on silica gel using first $n$-pentane (to remove the solvent) and then tert-butyl methyl ether as the eluent to afford 3-bromo2-methylbut-2-enoic acid $(E: Z=91: 9)(9,8 \mathrm{mg}, 22 \%)$ as a brown viscous oil.

The $E$ configuration was determined by NOESY measurements.

$\mathbf{R}_{\mathbf{f}}=0.40$ (n-pentane:Et $\left.{ }_{2} \mathrm{O}=1: 1\right)$. IR (ATR): $\tilde{v}=1184,1425,1661,2357,2928 \mathrm{~cm}^{-1}$. HRMS (APCI) for $\mathrm{C}_{5} \mathrm{H}_{7} \mathrm{BrO}_{2}{ }^{+}\left[\mathrm{M}^{+}\right]$: calculated 177.9629, found 177.9684 .

For (E)-3-bromo-2-methylbut-2-enoic acid (E-9):

${ }^{1} \mathrm{H}$ NMR (400 MHz, $\left.\mathrm{C}_{6} \mathrm{D}_{6}\right): \delta=1.70$ (q, $\left.J=0.8 \mathrm{~Hz}, 3 \mathrm{H}\right), 1.98(\mathrm{q}, J=0.6 \mathrm{~Hz}, 3 \mathrm{H}) \mathrm{ppm}$. (The $\mathrm{OH}$ signal was not identifiable.) ${ }^{13} \mathrm{C}$ NMR $\left(100 \mathrm{MHz}, \mathrm{C}_{6} \mathrm{D}_{6}\right): \delta=10.1,16.9$, 119.0, 159.2, $178.5 \mathrm{ppm}$.

For (Z)-3-bromo-2-methylbut-2-enoic acid (Z-9):

${ }^{1} \mathrm{H}$ NMR (400 MHz, $\left.\mathrm{C}_{6} \mathrm{D}_{6}\right): \delta=1.76(\mathrm{q}, J=0.8 \mathrm{~Hz}, 3 \mathrm{H}), 2.02(\mathrm{q}, J=0.6 \mathrm{~Hz}, 3 \mathrm{H}) \mathrm{ppm}$. (The $\mathrm{OH}$ signal was not identifiable.) ${ }^{13} \mathrm{C}$ NMR (100 MHz, $\left.\mathrm{C}_{6} \mathrm{D}_{6}\right)$ : Signals could not be identified due to the low quantity of material.

2-Methylbuta-2,3-dienoic acid (8) was prepared according to a reported procedure. ${ }^{\mathrm{S}}$ 


\section{Activation of Surrogate with $\left[\mathrm{Ph}_{3} \mathrm{C}\right]^{+}\left[\mathrm{B}\left(\mathrm{C}_{6} \mathrm{~F}_{5}\right)_{4}\right]^{-}$}

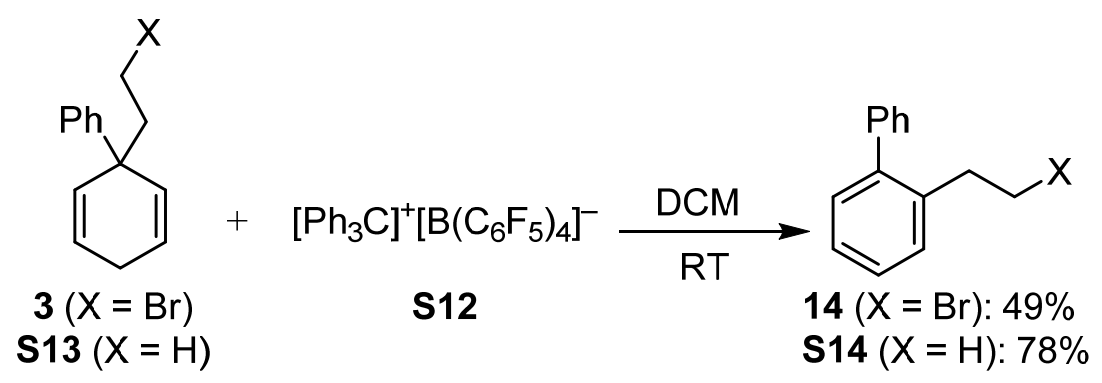

Scheme S7. Activation of cyclohexadienes with trityl salt

\subsection{2-(2-Bromoethyl)-1,1'-biphenyl (14)}<smiles>BrCCc1ccccc1-c1ccccc1</smiles>

\section{4}

$\mathrm{C}_{14} \mathrm{H}_{13} \mathrm{Br}$

$260.02 \mathrm{~g} / \mathrm{mol}$

In a glovebox, a Schlenk tube was charged with a solution of 1-(2-bromoethyl)-1,4dihydro-1,1'-biphenyl $(3,30.0 \mathrm{mg}, 0.120 \mathrm{mmol})$ in $\mathrm{CH}_{2} \mathrm{Cl}_{2}(1 \mathrm{~mL})$ and a suspension of $\left[\mathrm{Ph}_{3} \mathrm{C}\right]^{+}\left[\mathrm{B}\left(\mathrm{C}_{6} \mathrm{~F}_{5}\right)_{4}\right]^{-}\left(\mathrm{S} 12,120 \mathrm{mg}, 0.120 \mathrm{mmol}, 1.0\right.$ equiv) in $\mathrm{CH}_{2} \mathrm{Cl}_{2}(1 \mathrm{~mL})$ was added. The tube was sealed and removed from the glovebox and stirred under RT for 5 days. The reaction was quenched by filtration through a silica plug and the filtrate was concentrated under reduced pressure. The residue was purified by flash column chromatography on silica gel using cyclohexane as eluent to afford 2-(2-bromoethyl)1,1'-biphenyl $(14,26.0 \mathrm{mg})$ as a colorless viscous oil containing $44 \%$ of an inseparable byproduct that we assigned as triphenylmethane. This corresponds to a yield of $49 \%$ of 14 . Note: The reaction time, until full conversion of 3 , is determined with a parallel reaction in a $\mathrm{J}$ Young tube, monitored by ${ }^{1} \mathrm{H}$ NMR spectroscopy.

$\mathbf{R}_{\mathbf{f}}=0.70$ (cyclohexane). ${ }^{1} \mathrm{H}$ NMR (400 MHz, $\left.\mathrm{C}_{6} \mathrm{D}_{6}\right): \delta=3.16(\mathrm{t}, J=7.3 \mathrm{~Hz}, 2 \mathrm{H}), 3.37$ (t, $J=7.3 \mathrm{~Hz}, 2 \mathrm{H}), 7.29-7.34(\mathrm{~m}, 6 \mathrm{H}), 7.36-7.40(\mathrm{~m}, 1 \mathrm{H}), 7.42-7.45(\mathrm{~m}, 2 \mathrm{H}) \mathrm{ppm}$. ${ }^{13} \mathrm{C}$ NMR (100 MHz, $\left.\mathrm{C}_{6} \mathrm{D}_{6}\right): \delta=32.8,37.0,127.2,127.6,128.0,129.5$ (2 carbons), $130.0,130.7,136.8,141.6,142.7$ ppm. 


\subsection{1-Ethyl-1,4-dihydro-1,1'-biphenyl (S13)}<smiles>CCCC1(c2ccccc2)C=CCC=C1</smiles>

S13

$\mathrm{C}_{14} \mathrm{H}_{16}$

$184.12 \mathrm{~g} / \mathrm{mol}$

A three-necked round-bottomed flask equipped with a dry ice condenser and an overhead stirrer was purged with $\mathrm{N}_{2}$ for 15 min before being placed in a dry ice/acetone bath. Ammonia (approx. $50 \mathrm{~mL}$ ) was condensed, and then lithium (182 $\mathrm{mg}, 26.0 \mathrm{mmol}, 4.0$ equiv) was added portionwise over a period of $30 \mathrm{~min}$ to result in a dark blue suspension. A solution of biphenyl $(\mathbf{S 9}, 1.00 \mathrm{~g}, 6.49 \mathrm{mmol})$ in THF $(5 \mathrm{~mL})$ was added dropwise over a period of $30 \mathrm{~min}$ to result in a brick red suspension. The suspension was stirred at $-78{ }^{\circ} \mathrm{C}$ for a further 60 min. A solution of $\mathrm{CH}_{3} \mathrm{CH}_{2} \mathrm{I}(2.08$ $\mathrm{mL}, 4.05 \mathrm{~g}, 26.0 \mathrm{mmol}, 4.0$ equiv) in THF (50 mL) was then added dropwise over a period of $10 \mathrm{~min}$. The resulting pale yellow suspension was stirred overnight to facilitate the evaporation of ammonia. Water $(100 \mathrm{~mL})$ was added and the mixture was extracted with tert-butyl methyl ether $(3 \times 100 \mathrm{~mL})$. The combined organic phases were washed with brine $(100 \mathrm{~mL})$, dried over $\mathrm{MgSO}_{4}$ and concentrated under reduced pressure. The residue was purified by flash column chromatography on silica gel using cyclohexane as eluent to afford 1-ethyl-1,4-dihydro-1,1'-biphenyl (S13, $950 \mathrm{mg}, 79 \%)$ as a colorless oil.

$\mathbf{R}_{\mathbf{f}}=0.90$ (cyclohexane). IR (ATR): $\tilde{v}=694,759,945,1445,1490,2923 \mathrm{~cm}^{-1}$. HRMS (El) for $\mathrm{C}_{14} \mathrm{H}_{16}{ }^{+}\left[\mathrm{M}^{+}\right]$: calculated 148.1246, found 148.1255. ${ }^{1} \mathrm{H}$ NMR (400 MHz, $\left.\mathrm{CDCl}_{3}\right): \delta=0.79(\mathrm{t}, J=7.5,3 \mathrm{H}), 1.75(\mathrm{q}, J=7.5,2 \mathrm{H}), 2.51-2.65(\mathrm{~m}, 2 \mathrm{H}), 5.49(\mathrm{dt}, J=$ $10.4,1.5 \mathrm{~Hz}, 2 \mathrm{H}), 5.77$ (dt, $J=10.4,3.3 \mathrm{~Hz}, 2 \mathrm{H}), 7.07$ (tt, $J=7.2,1.9 \mathrm{~Hz}, 1 \mathrm{H}), 7.18-$ $7.23(\mathrm{~m}, 2 \mathrm{H}), 7.26-7.30(\mathrm{~m}, 2 \mathrm{H}) \mathrm{ppm} .{ }^{13} \mathrm{C}$ NMR $\left(100 \mathrm{MHz}, \mathrm{CDCl}_{3}\right): \delta=9.4,26.3$, $32.5,44.6,123.8,125.9,126.8,128.3,132.5,148.5$ ppm. 


\subsection{2-Ethyl-1,1'-biphenyl (S14)}

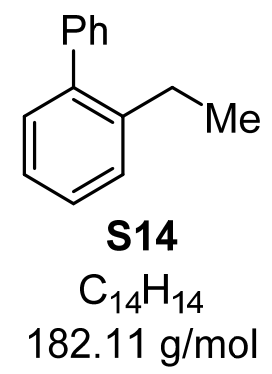

In a glovebox, a Schlenk tube was charged with a solution of 1-ethyl-1,4-dihydro-1,1'biphenyl (S13, $20.0 \mathrm{mg}, 11.0 \mathrm{mmol})$ in $\mathrm{CH}_{2} \mathrm{Cl}_{2}(1 \mathrm{~mL})$, and a suspension of $\left[\mathrm{Ph}_{3} \mathrm{C}\right]^{+}\left[\mathrm{B}\left(\mathrm{C}_{6} \mathrm{~F}_{5}\right)_{4}\right]^{-}\left(\mathrm{S} 12,120 \mathrm{mg}, 0.120 \mathrm{mmol}, 1.0\right.$ equiv) in $\mathrm{CH}_{2} \mathrm{Cl}_{2}(1 \mathrm{~mL})$ was added. The tube was sealed and removed from the glovebox and stirred under RT for 3 days. The reaction was quenched by filtration through a silica plug and the filtrate was concentrated under reduced pressure. The residue was purified by flash column chromatography on silica gel using cyclohexane as eluent to afford 2-ethyl-1,1'biphenyl (S14, $15.4 \mathrm{mg}, 78 \%$ ), as a colorless oil. Note: The reaction time, until full conversion of S13, was determined with a parallel reaction in a $\mathrm{J}$ Young tube, monitored by ${ }^{1} \mathrm{H}$ NMR spectroscopy.

$\mathbf{R}_{\mathbf{f}}=0.70$ (cyclohexane). IR (ATR): $\tilde{v}=702,751,1478,2360,2926,2964 \mathrm{~cm}^{-1}$. HRMS (APCl) for $\mathrm{C}_{14} \mathrm{H}_{14}{ }^{+}\left[\mathrm{M}^{+}\right]$: calculated 182.1096, found 182.1092. ${ }^{1} \mathrm{H}$ NMR (400 $\left.\mathrm{MHz}, \mathrm{C}_{6} \mathrm{D}_{6}\right): \delta=1.11(\mathrm{t}, J=7.5 \mathrm{~Hz}, 3 \mathrm{H}), 2.62(\mathrm{q}, J=7.5 \mathrm{~Hz}, 2 \mathrm{H}), 7.19-7.27(\mathrm{~m}, 2 \mathrm{H})$, 7.32-7.36 (m, 4H), 7.37-7.39 (m, 1H), 7.42-7.46 (m, 2H) ppm. ${ }^{13} \mathrm{C}$ NMR $(100 \mathrm{MHz}$, $\left.\mathrm{C}_{6} \mathrm{D}_{6}\right): \delta=15.8,26.5,125.9,127.1,127.8,128.4,129.0,129.6,130.3,142.0,142.1$, 142.4 ppm. 


\section{$5 \quad$ NMR Spectra}

Scheme S8. ${ }^{1} \mathrm{H}$ NMR spectrum $\left(500 \mathrm{MHz}, \mathrm{CDCl}_{3}, 298 \mathrm{~K}\right)$ of (1-methylcyclohexa-2,5-dien-1-yl)methanol (S2)<smiles>CC1(CO)C=CCC=C1</smiles>

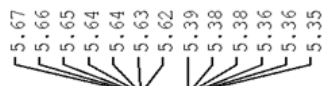

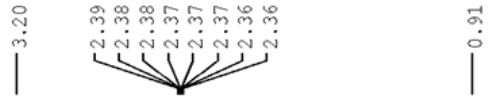

Me

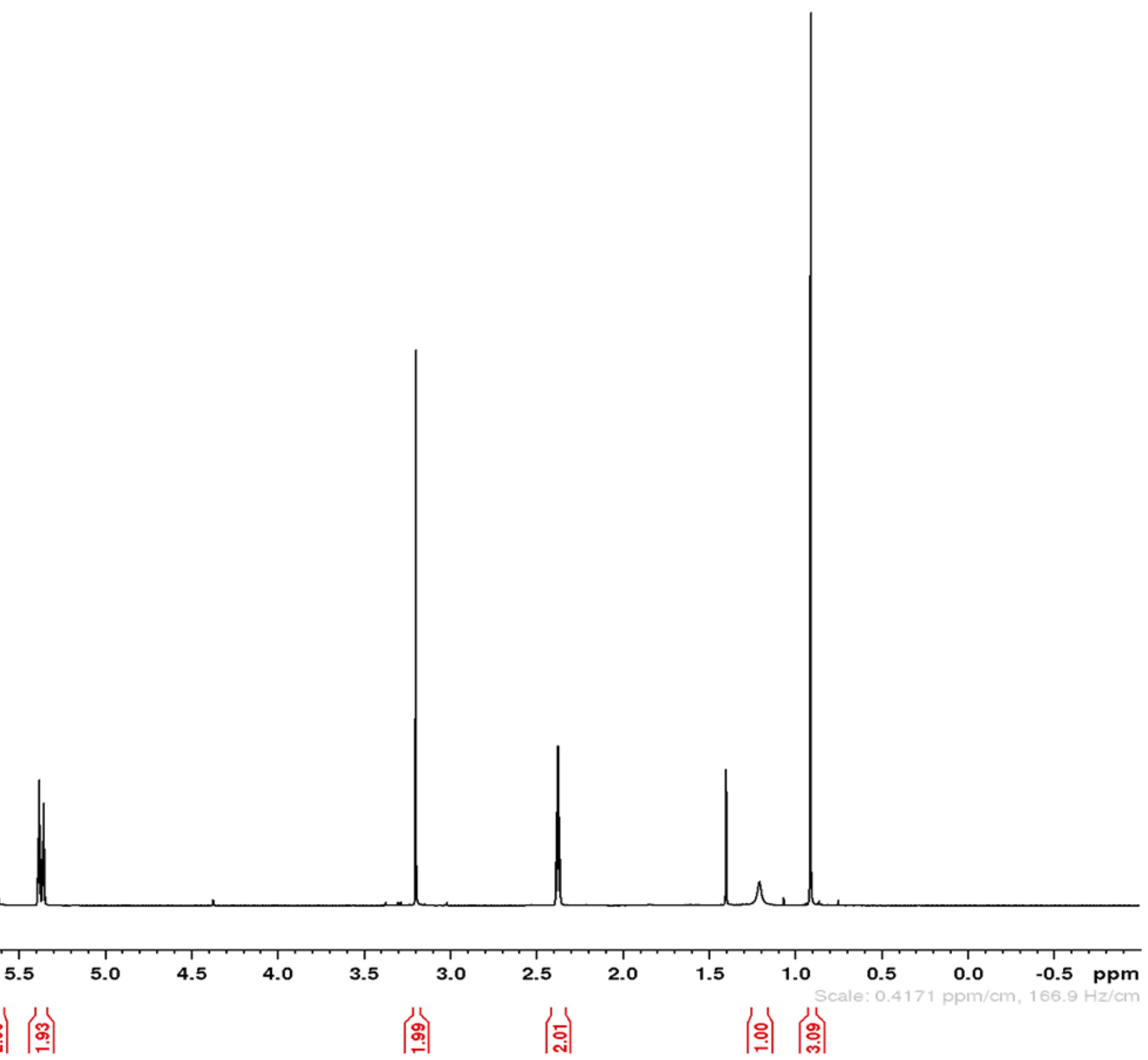


Scheme S9. ${ }^{13} \mathrm{C}\left\{{ }^{1} \mathrm{H}\right\}$ NMR (125 MHz, CDCl, $\left.298 \mathrm{~K}\right)$ of (1-methylcyclohexa-2,5-dien-1-yl)methanol (S2)

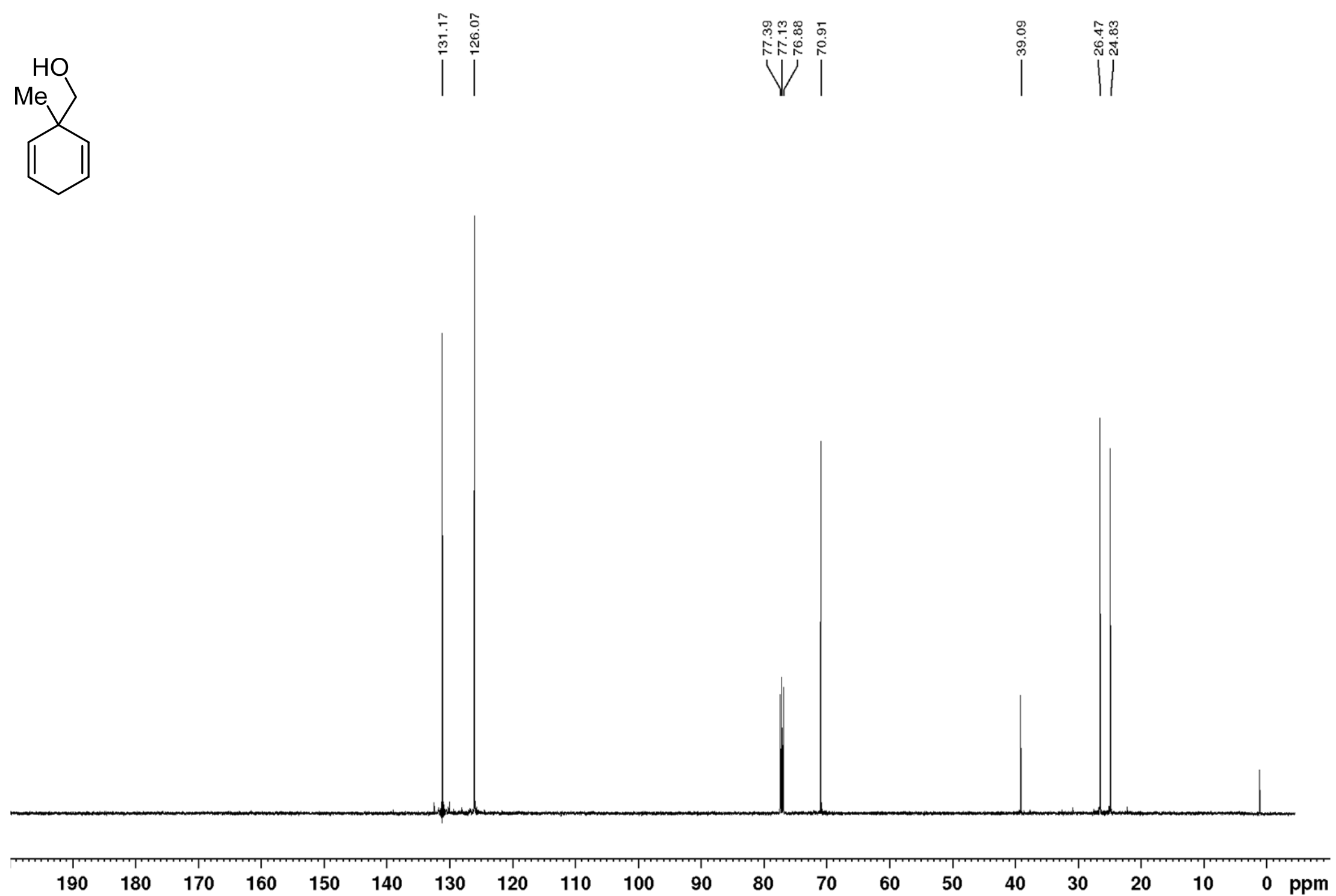


Supporting Information for Organic Letters

S49

Scheme S10. ${ }^{1} \mathrm{H}$ NMR spectrum $\left(400 \mathrm{MHz}, \mathrm{CDCl}_{3}, 298 \mathrm{~K}\right)$ of 1-methylcyclohexa-2,5-diene-1-carbaldehyde (S3)<smiles>CC1(C=O)C=CCC=C1</smiles>
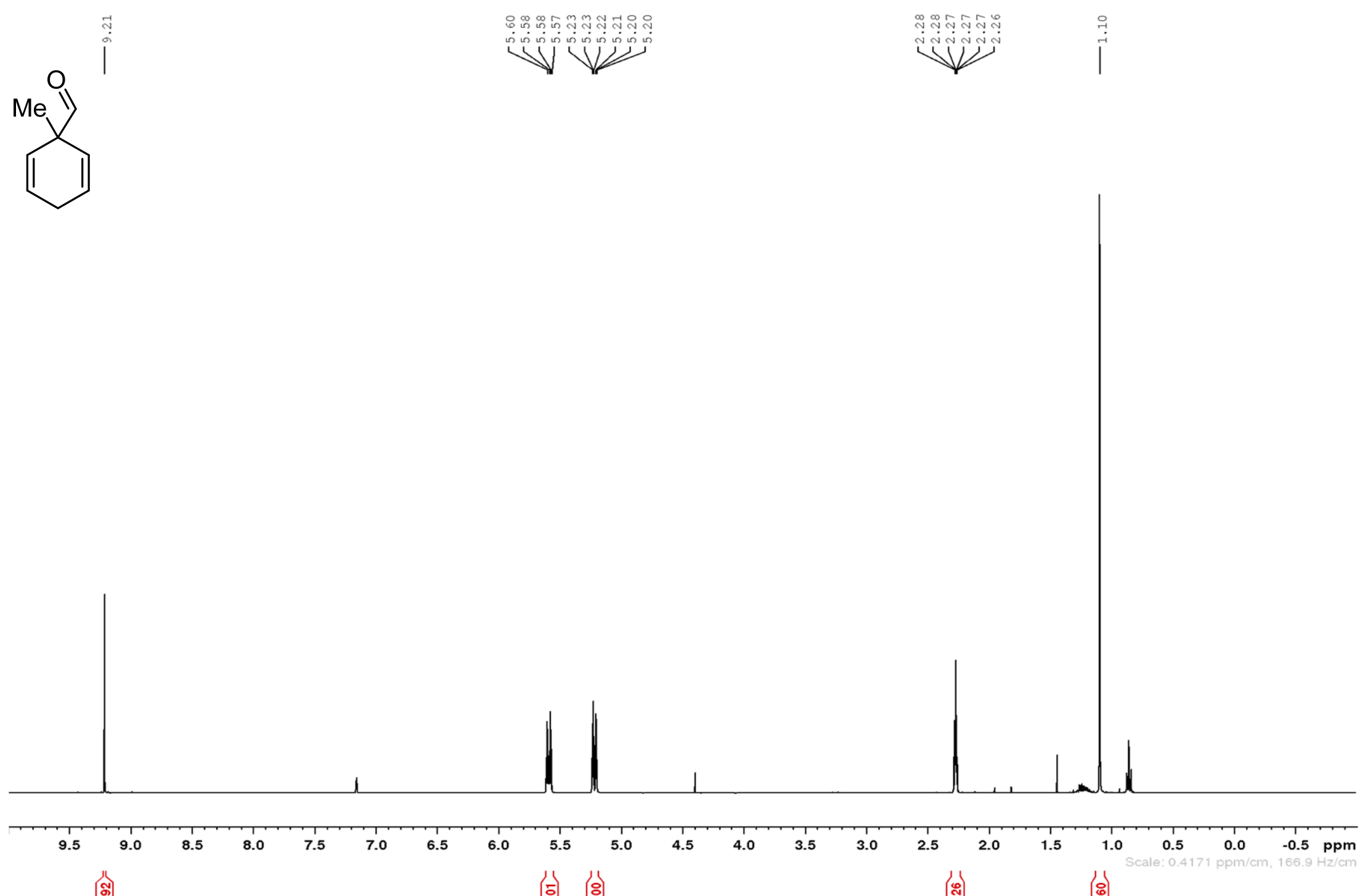

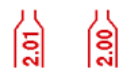

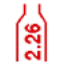

(:) 
Scheme S11. ${ }^{13} \mathrm{C}\left\{{ }^{1} \mathrm{H}\right\} \mathrm{NMR}\left(100 \mathrm{MHz}, \mathrm{CDCl}_{3}, 298 \mathrm{~K}\right)$ of 1-methylcyclohexa-2,5-diene-1-carbaldehyde (S3)
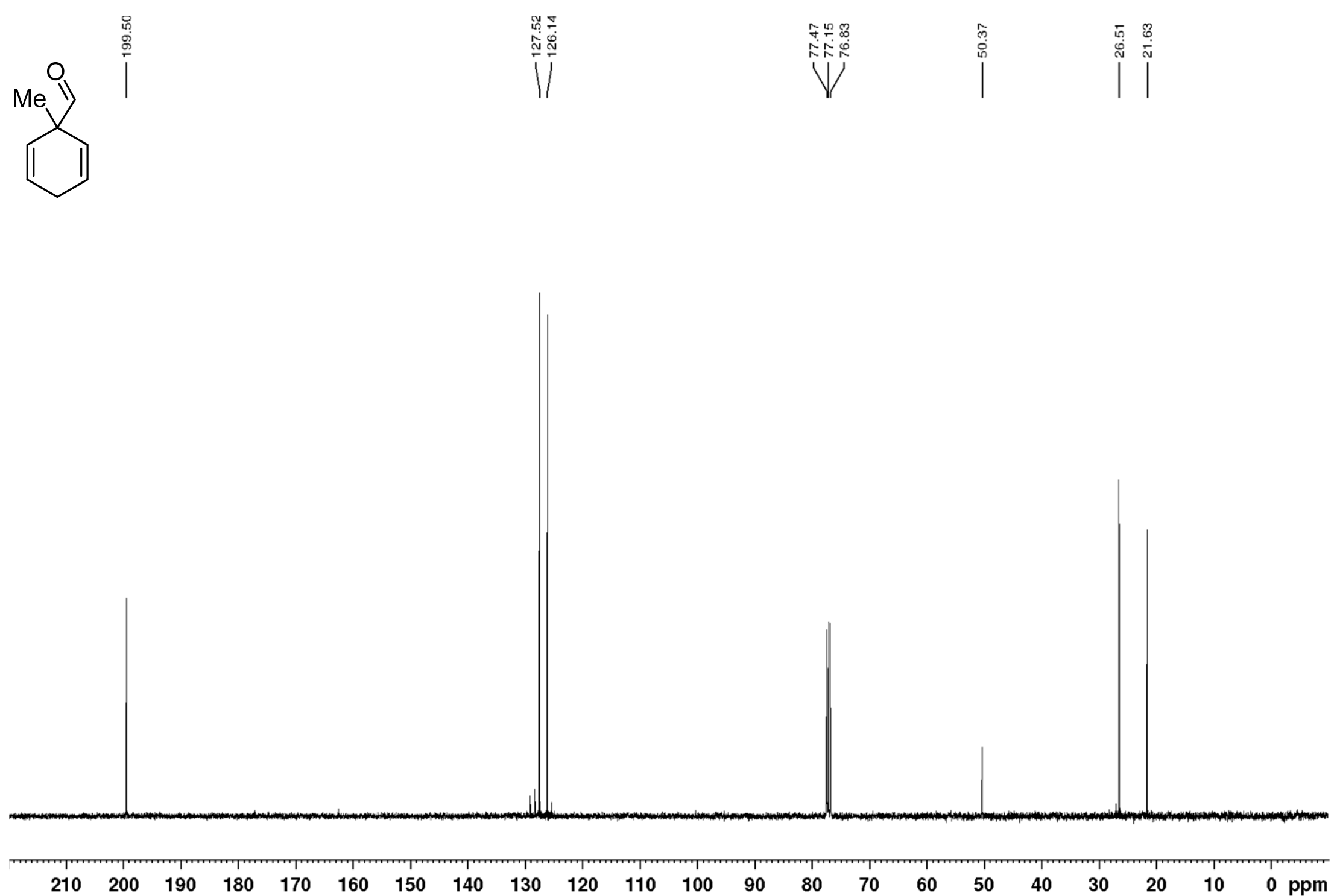
Scheme S12. ${ }^{1} \mathrm{H}$ NMR spectrum (500 MHz, $\left.\mathrm{CD}_{2} \mathrm{Cl}_{2}, 298 \mathrm{~K}\right)$ of 2-(1-methylcyclohexa-2,5-dien-1-yl)acetaldehyde (S4)

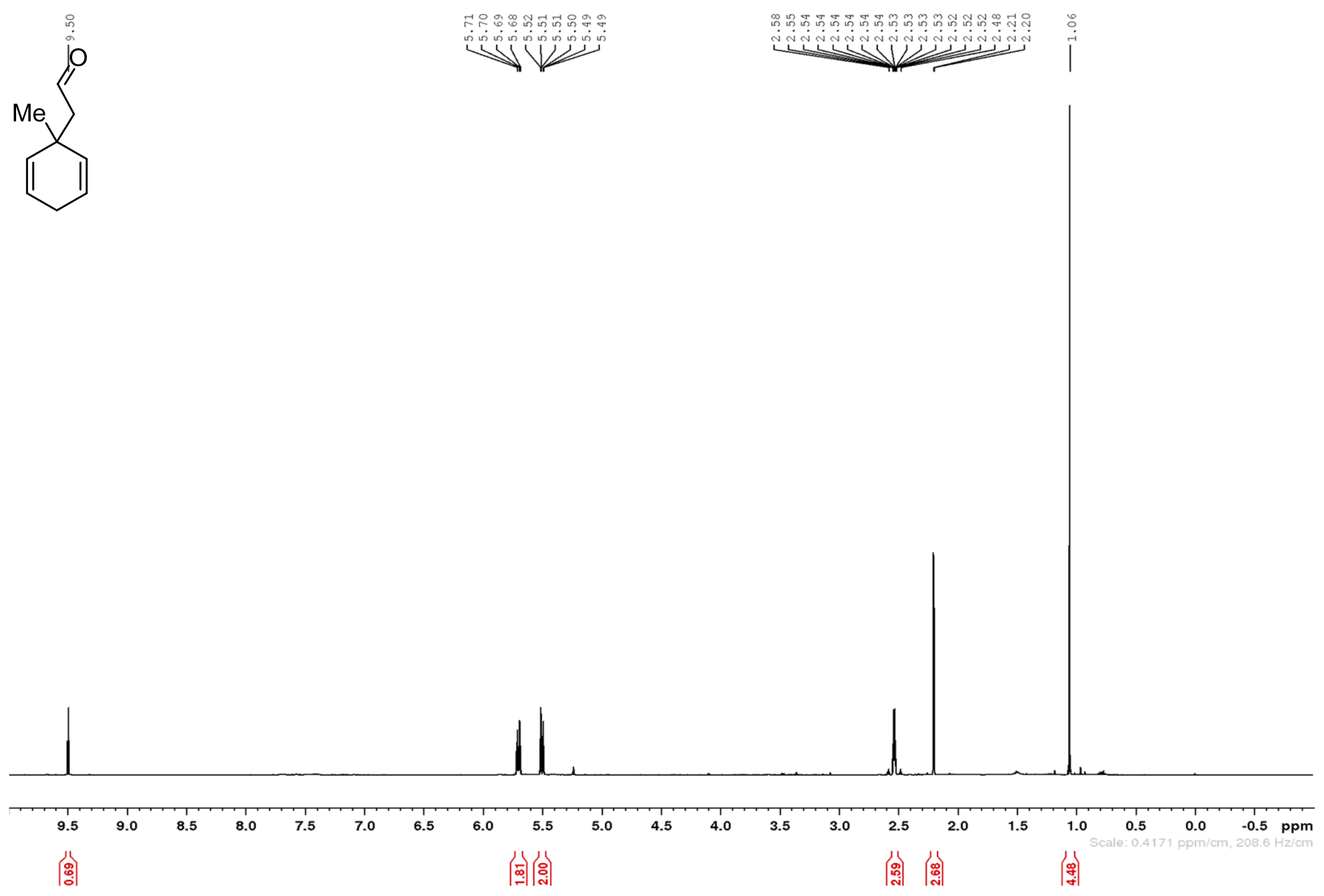


Scheme S13. ${ }^{13} \mathrm{C}\left\{{ }^{1} \mathrm{H}\right\}$ NMR (125 MHz, $\left.\mathrm{CD}_{2} \mathrm{Cl}_{2}, 298 \mathrm{~K}\right)$ of 2-(1-methylcyclohexa-2,5-dien-1-yl)acetaldehyde (S4)
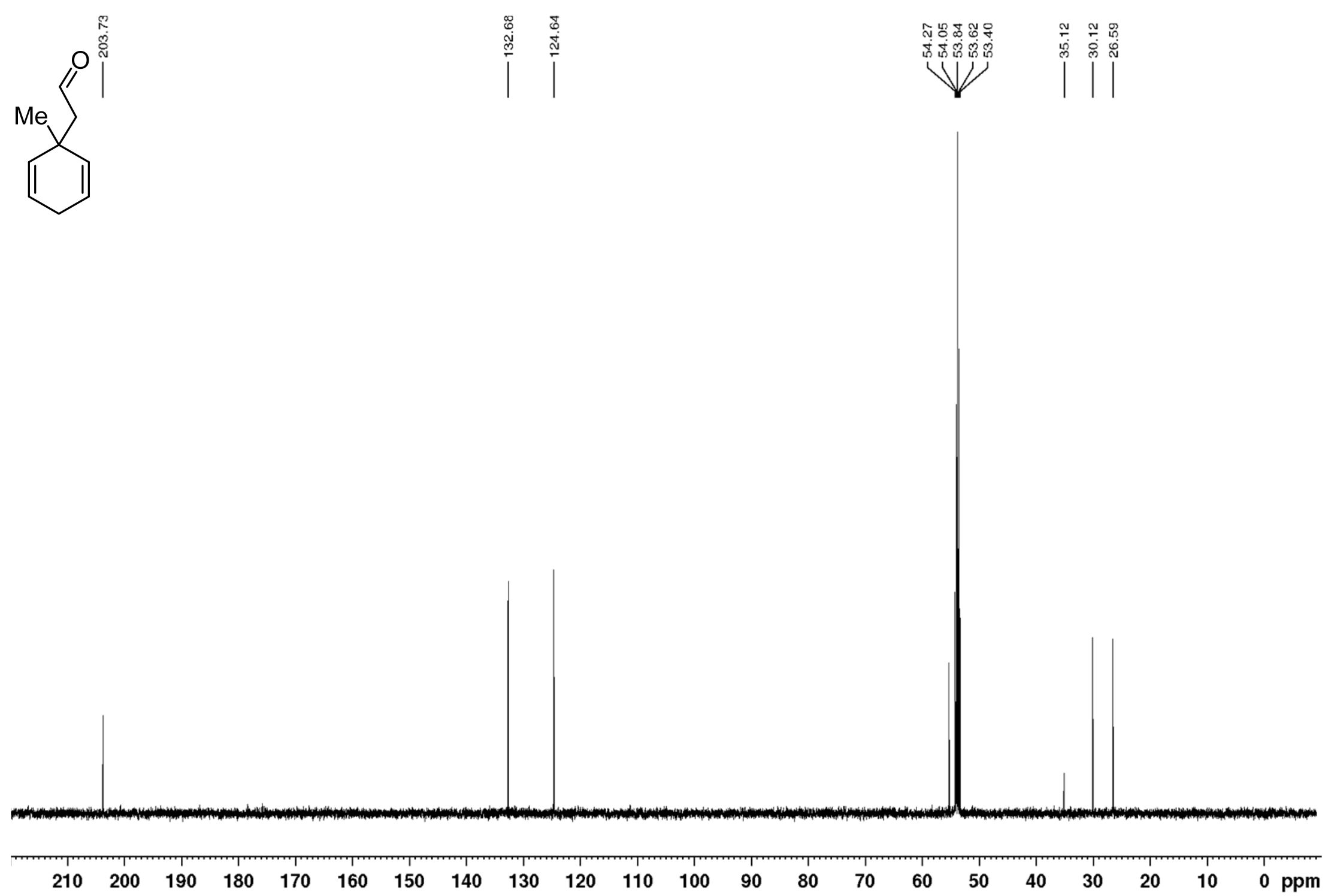
Scheme S14. ${ }^{1} \mathrm{H}$ NMR spectrum $\left(400 \mathrm{MHz}, \mathrm{CD}_{2} \mathrm{Cl}_{2}, 298 \mathrm{~K}\right)$ of 2-(1-methylcyclohexa-2,5-dien-1-yl)ethan-1-ol (S5)
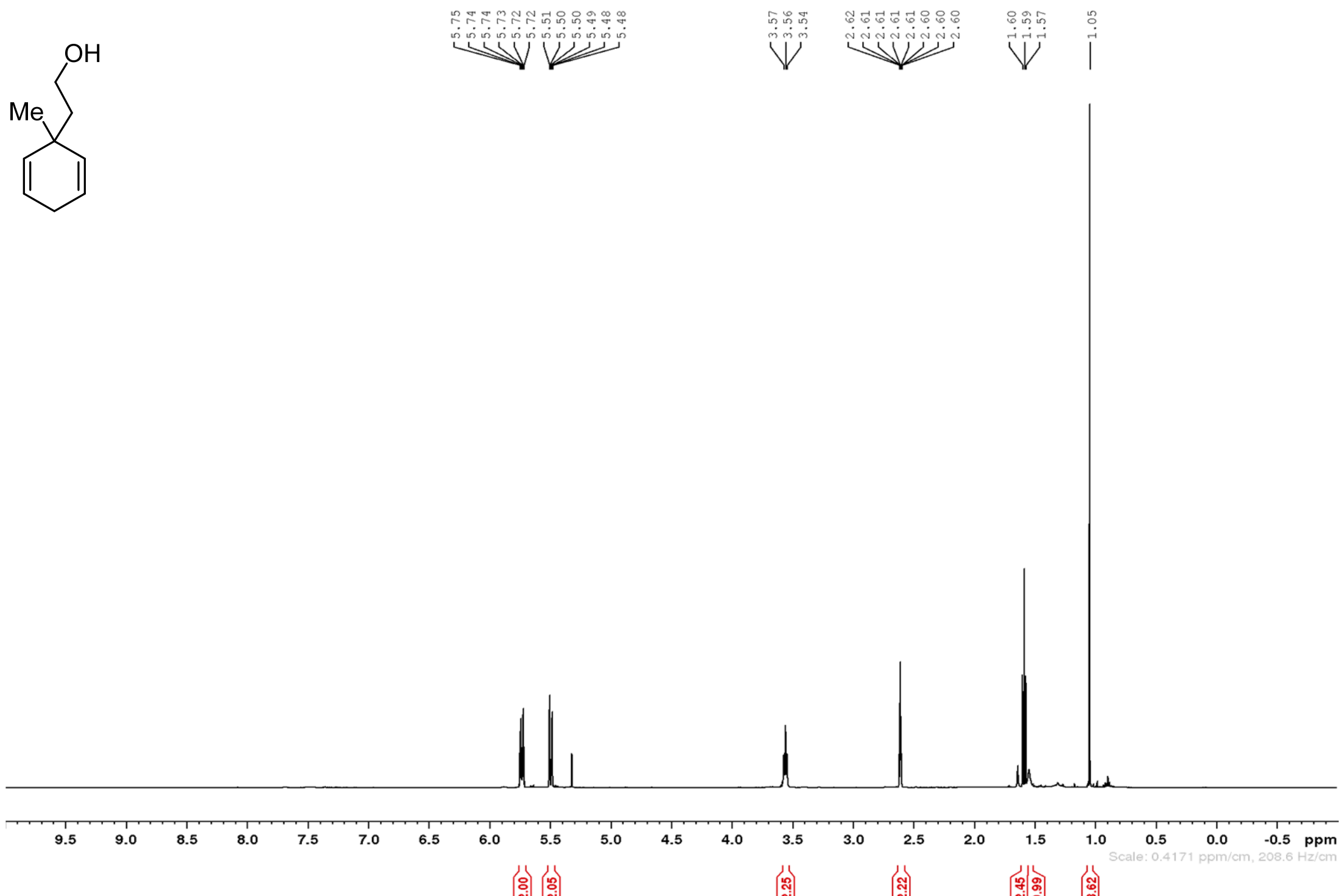
Scheme S15. ${ }^{13} \mathrm{C}\left\{{ }^{1} \mathrm{H}\right\}$ NMR (100 MHz, $\left.\mathrm{CD}_{2} \mathrm{Cl}_{2}, 298 \mathrm{~K}\right)$ of 2-(1-methylcyclohexa-2,5-dien-1-yl)ethan-1-ol (S5)

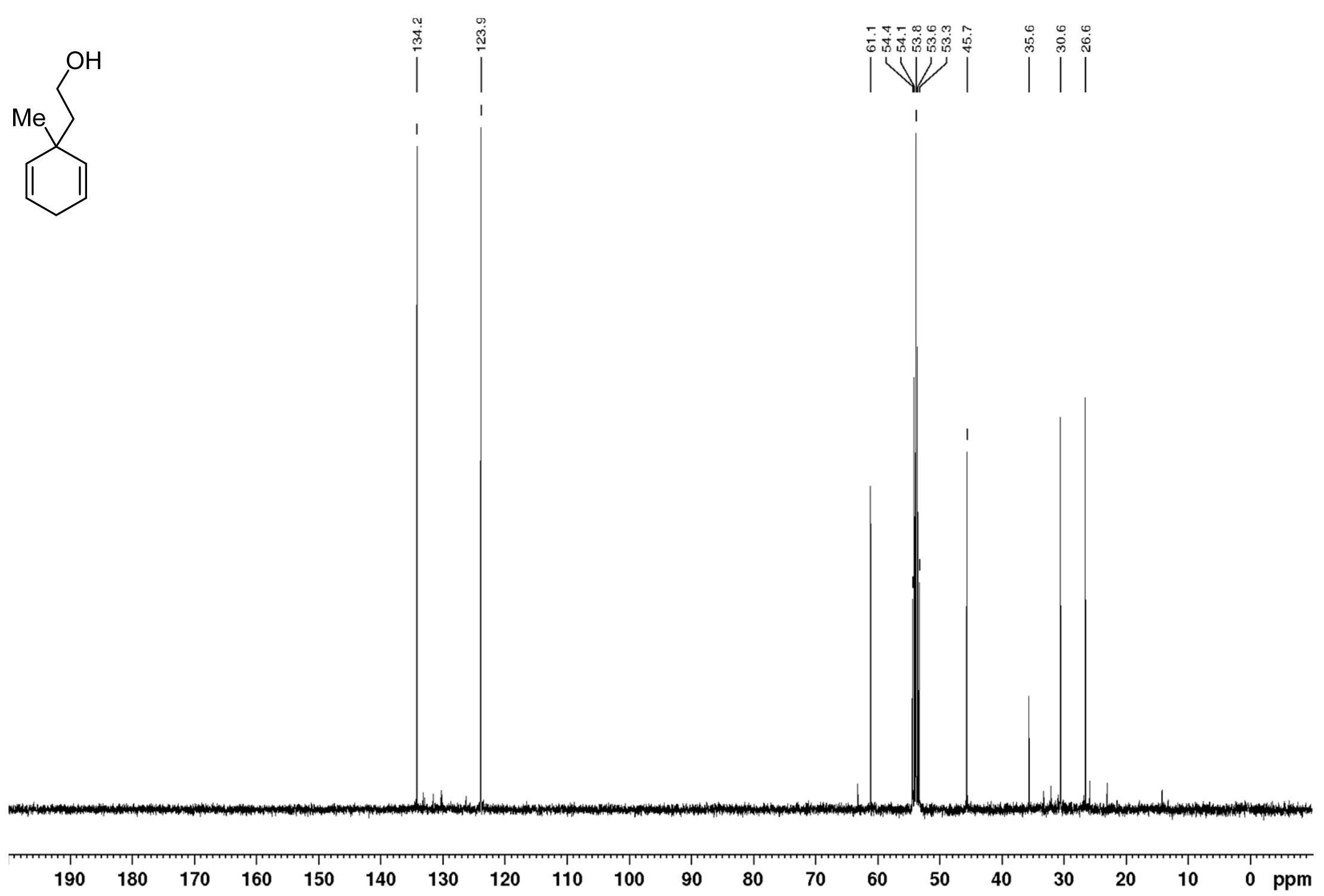


Scheme S16. ${ }^{1} \mathrm{H}$ NMR spectrum (500 MHz, $\mathrm{CD}_{2} \mathrm{Cl}_{2}, 298 \mathrm{~K}$ ) of 3-(2-bromoethyl)-3-methylcyclohexa-1,4-diene (1)
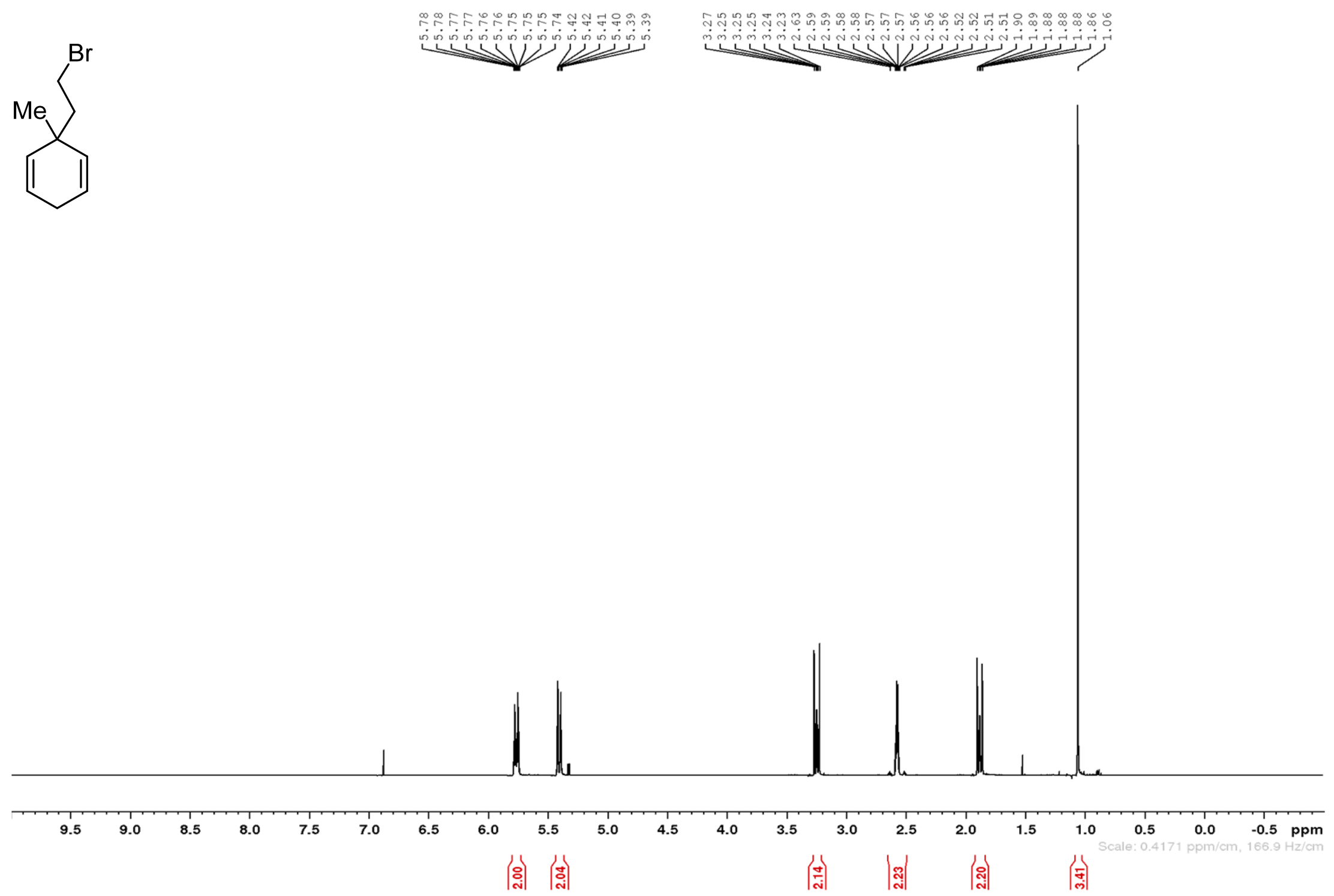
Scheme S17. ${ }^{13} \mathrm{C}\left\{{ }^{1} \mathrm{H}\right\}$ NMR (125 MHz, $\mathrm{CD}_{2} \mathrm{Cl}_{2}, 298 \mathrm{~K}$ ) of 3-(2-bromoethyl)-3-methylcyclohexa-1,4-diene (1)<smiles>CC1(CCBr)C=CCC=C1</smiles>
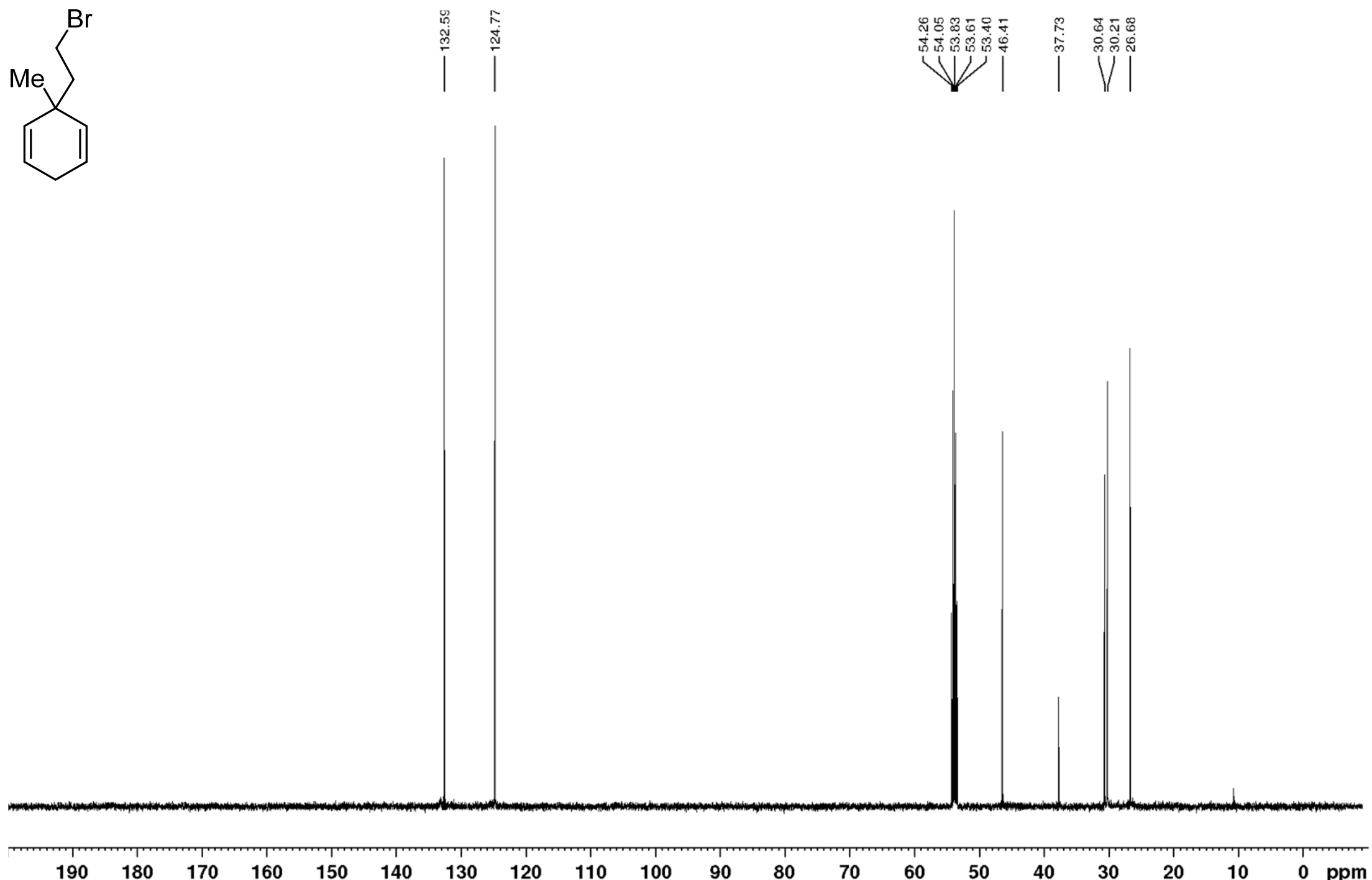
Scheme S18. ${ }^{1} \mathrm{H}$ NMR spectrum (400 MHz, $\left.\mathrm{C}_{6} \mathrm{D}_{6}, 298 \mathrm{~K}\right)$ of 2-(1,3,5-trimethylcyclohexa-2,5-dien-1-yl)ethan-1-ol (S7)<smiles>CC1=CC(C)(CO)C=C(C)C1</smiles>

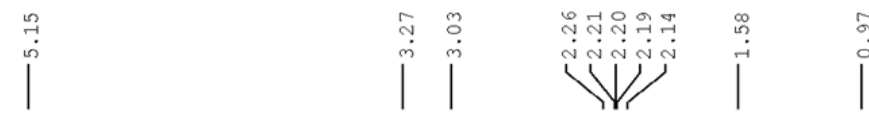

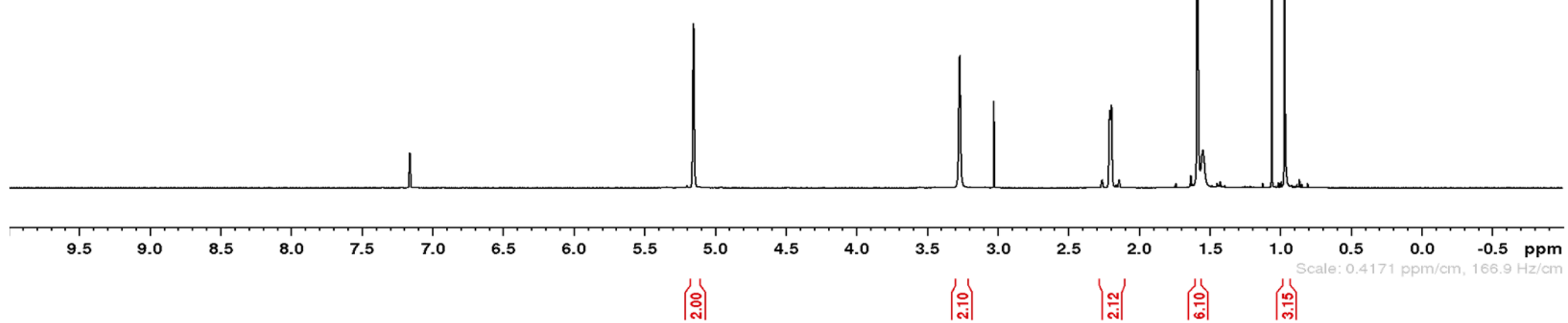


Scheme S19. ${ }^{13} \mathrm{C}\left\{{ }^{1} \mathrm{H}\right\}$ NMR (100 MHz, $\left.\mathrm{C}_{6} \mathrm{D}_{6}, 298 \mathrm{~K}\right)$ of 2-(1,3,5-trimethylcyclohexa-2,5-dien-1-yl)ethan-1-ol (S7)
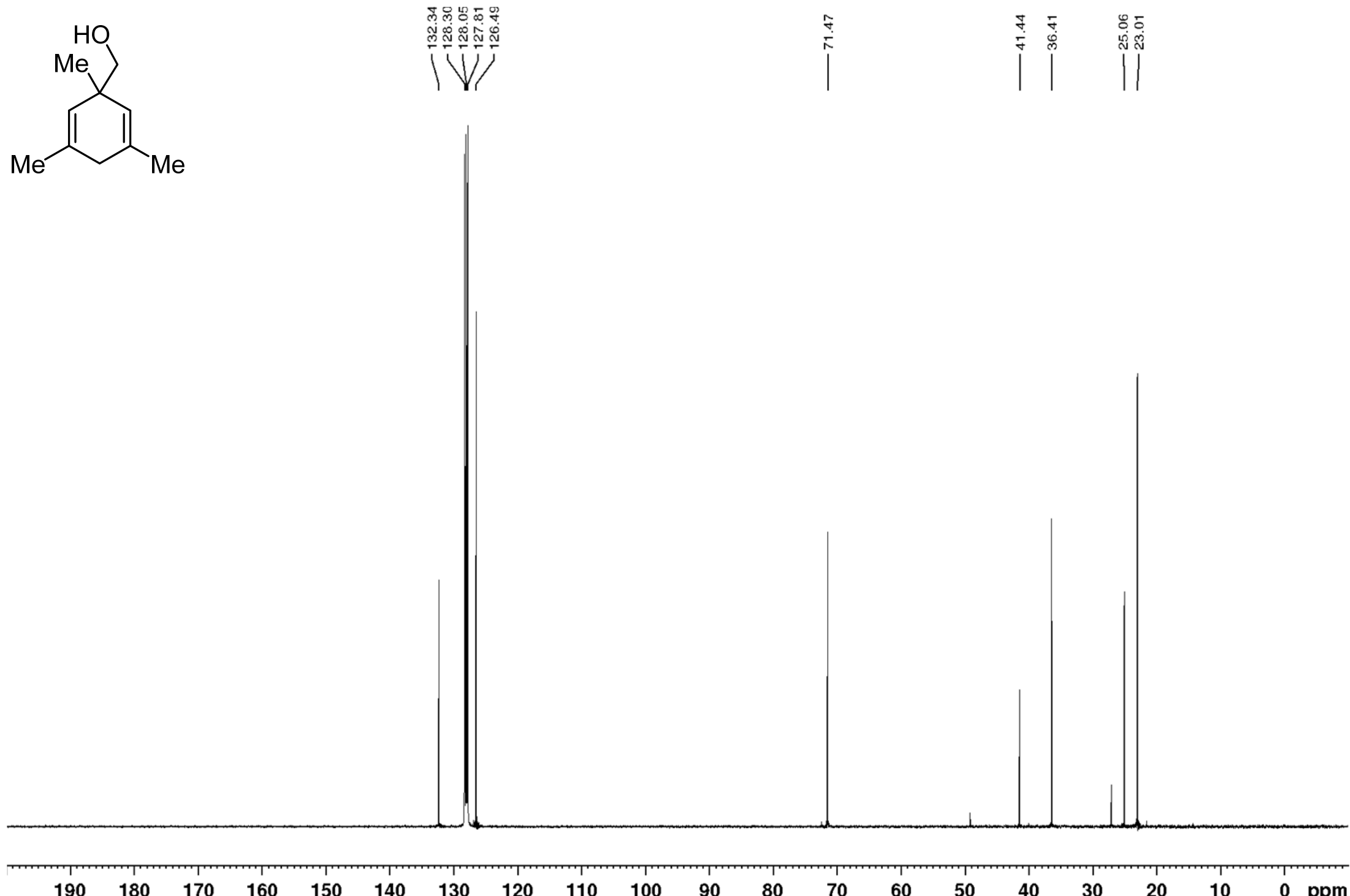
Scheme S20. ${ }^{1} \mathrm{H}$ NMR spectrum (400 MHz, $\left.\mathrm{C}_{6} \mathrm{D}_{6}, 298 \mathrm{~K}\right)$ of 2-(1,3,5-trimethylcyclohexa-2,5-dien-1-yl)ethan-1-ol (S8)<smiles>CC1=CC(C)(CCO)C=C(C)C1</smiles>

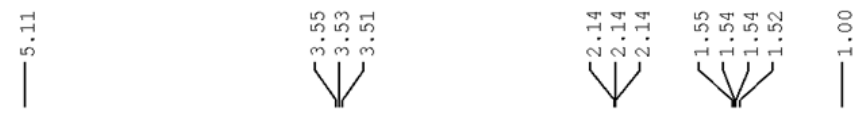

$\mathrm{Me}$

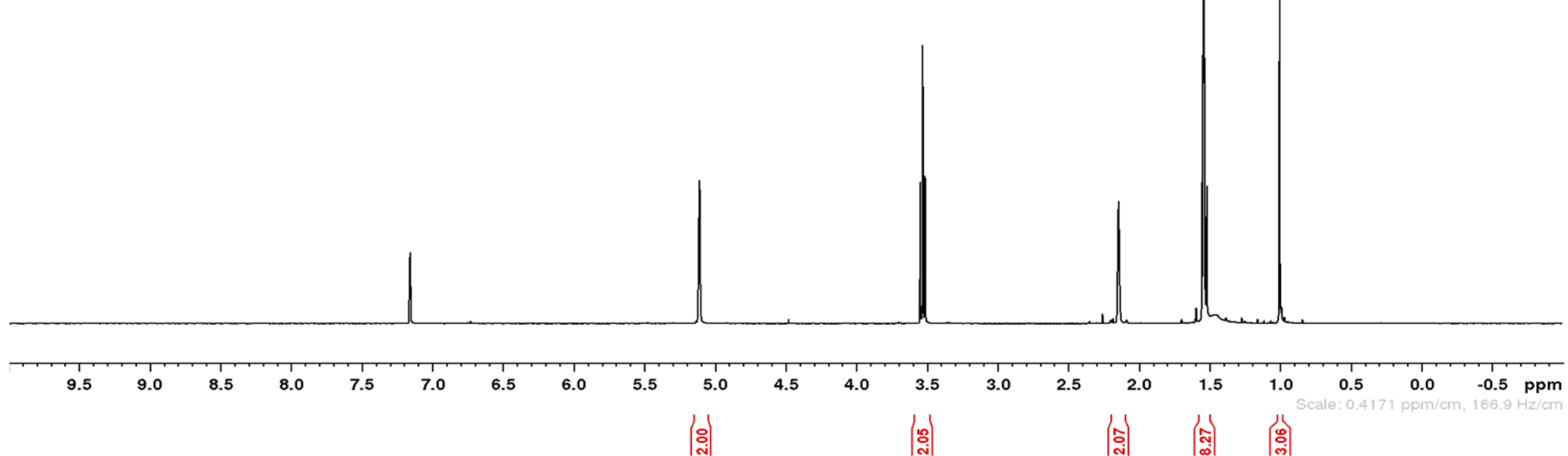


Scheme S21. ${ }^{13} \mathrm{C}\left\{{ }^{1} \mathrm{H}\right\}$ NMR (100 MHz, $\left.\mathrm{C}_{6} \mathrm{D}_{6}, 298 \mathrm{~K}\right)$ of 2-(1,3,5-trimethylcyclohexa-2,5-dien-1-yl)ethan-1-ol (S8)
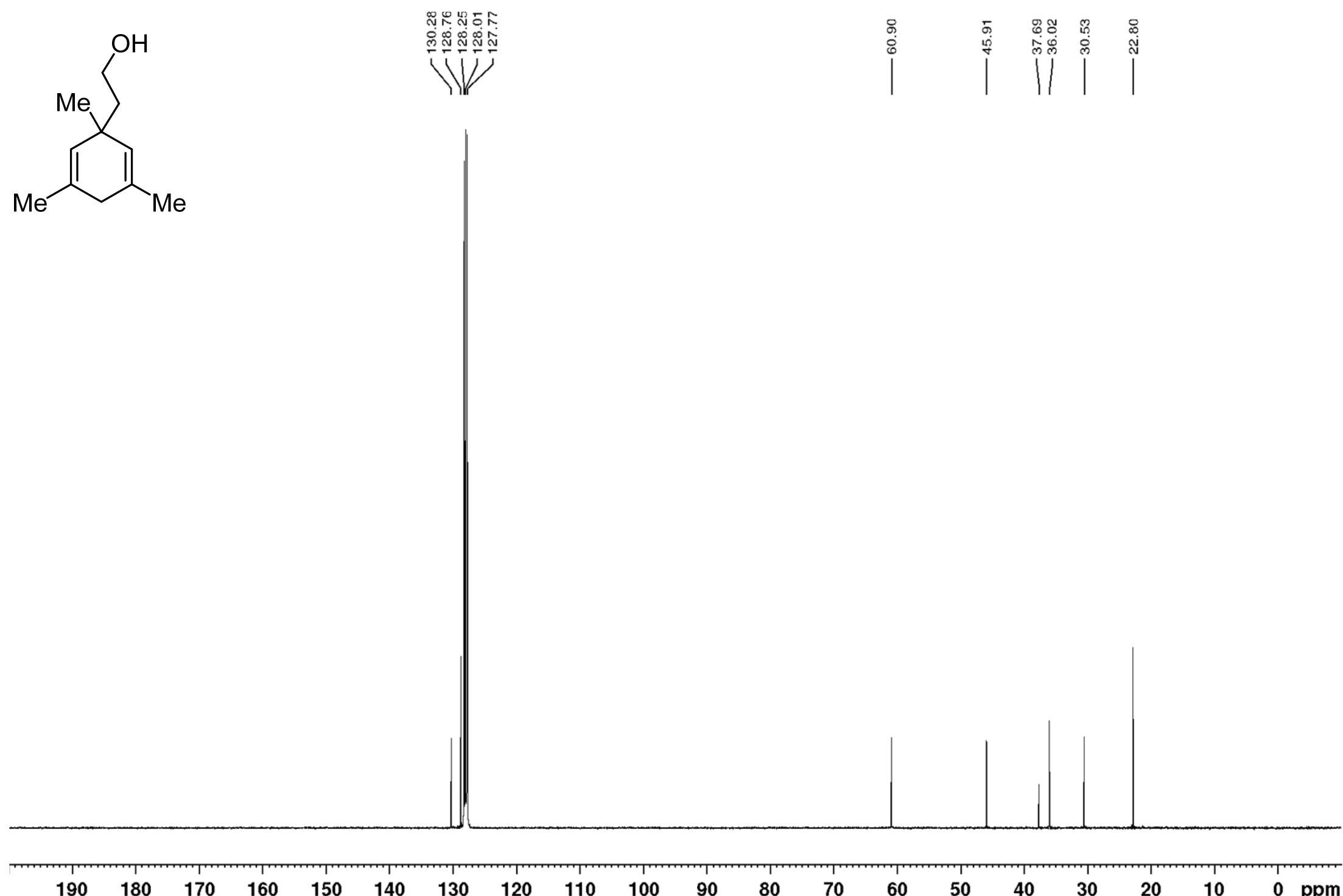
Scheme S22. ${ }^{1} \mathrm{H}$ NMR spectrum (400 MHz, $\mathrm{C}_{6} \mathrm{D}_{6}, 298 \mathrm{~K}$ ) of 3-(2-bromoethyl)-1,3,5-trimethylcyclohexa-1,4-diene (2)<smiles>CC1=CC(C)(CCBr)C=C(C)C1</smiles>
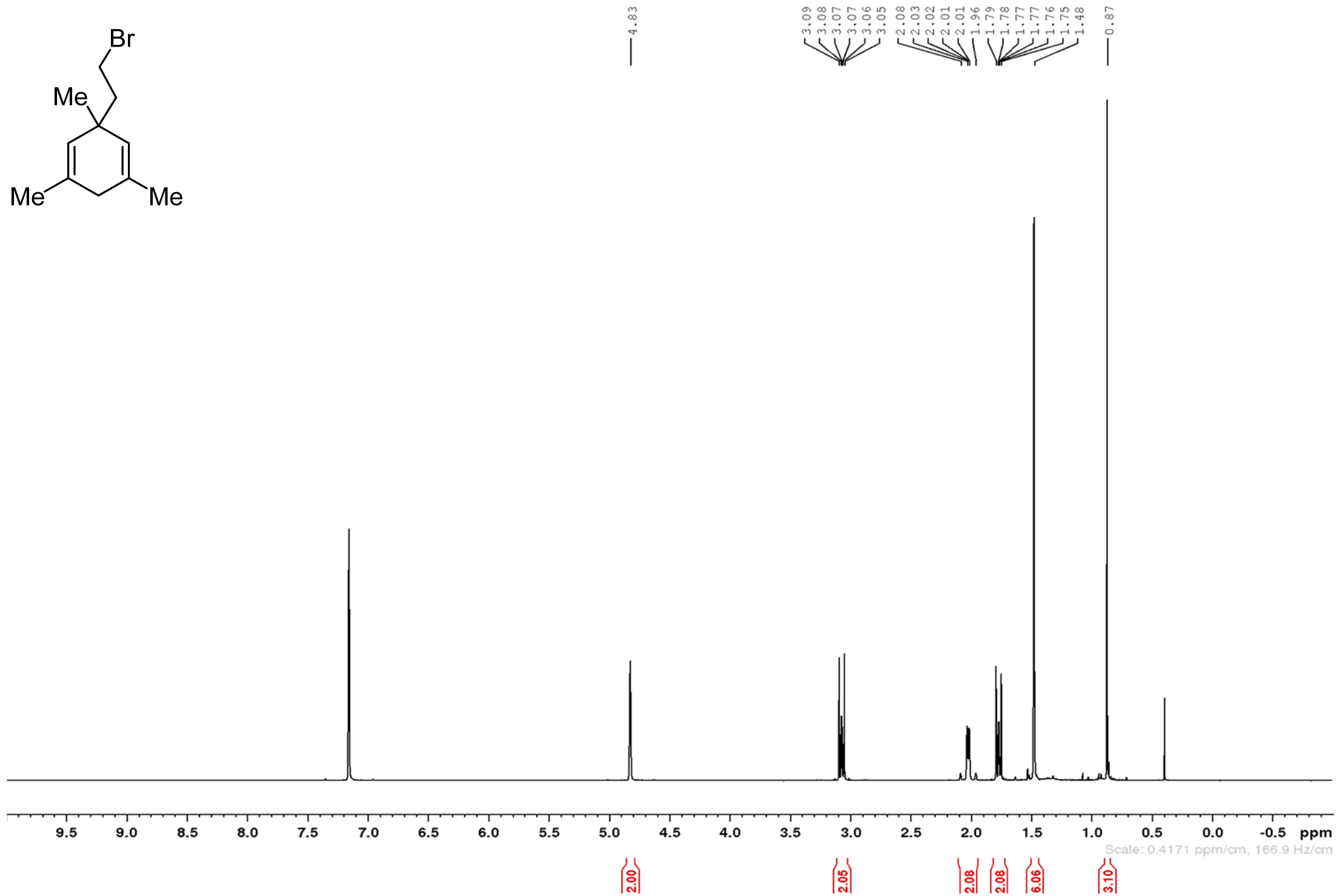
Scheme S23. ${ }^{13} \mathrm{C}\left\{{ }^{1} \mathrm{H}\right\}$ NMR (100 MHz, $\left.\mathrm{C}_{6} \mathrm{D}_{6}, 298 \mathrm{~K}\right)$ of 3-(2-bromoethyl)-1,3,5-trimethylcyclohexa-1,4-diene (2)

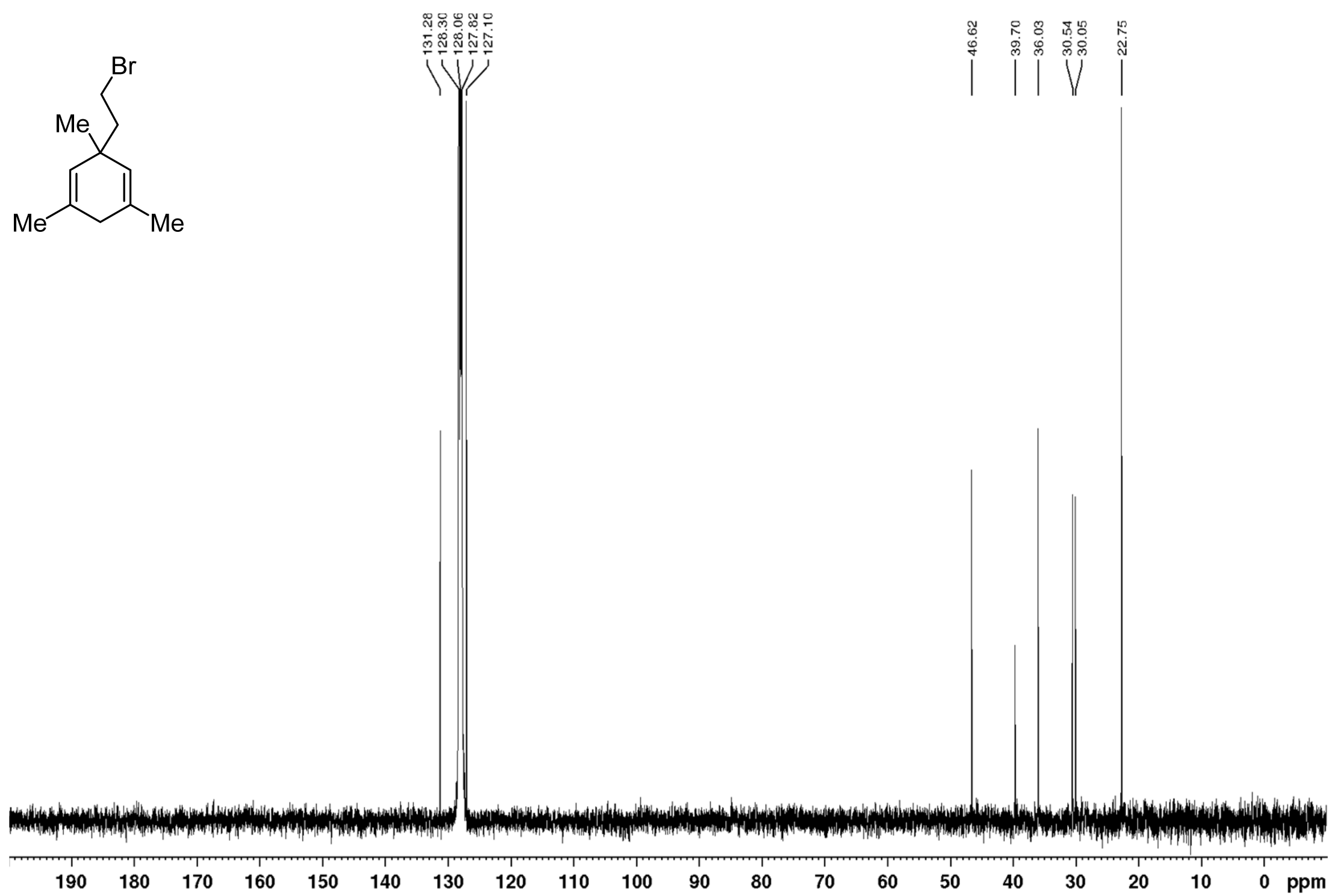


Scheme S24. ${ }^{1} \mathrm{H}$ NMR spectrum $\left(400 \mathrm{MHz}, \mathrm{C}_{6} \mathrm{D}_{6}, 298 \mathrm{~K}\right)$ of ethyl 2-([1,1'-biphenyl]-1(4H)-yl)acetate (\# = cyclohexane) (S10)
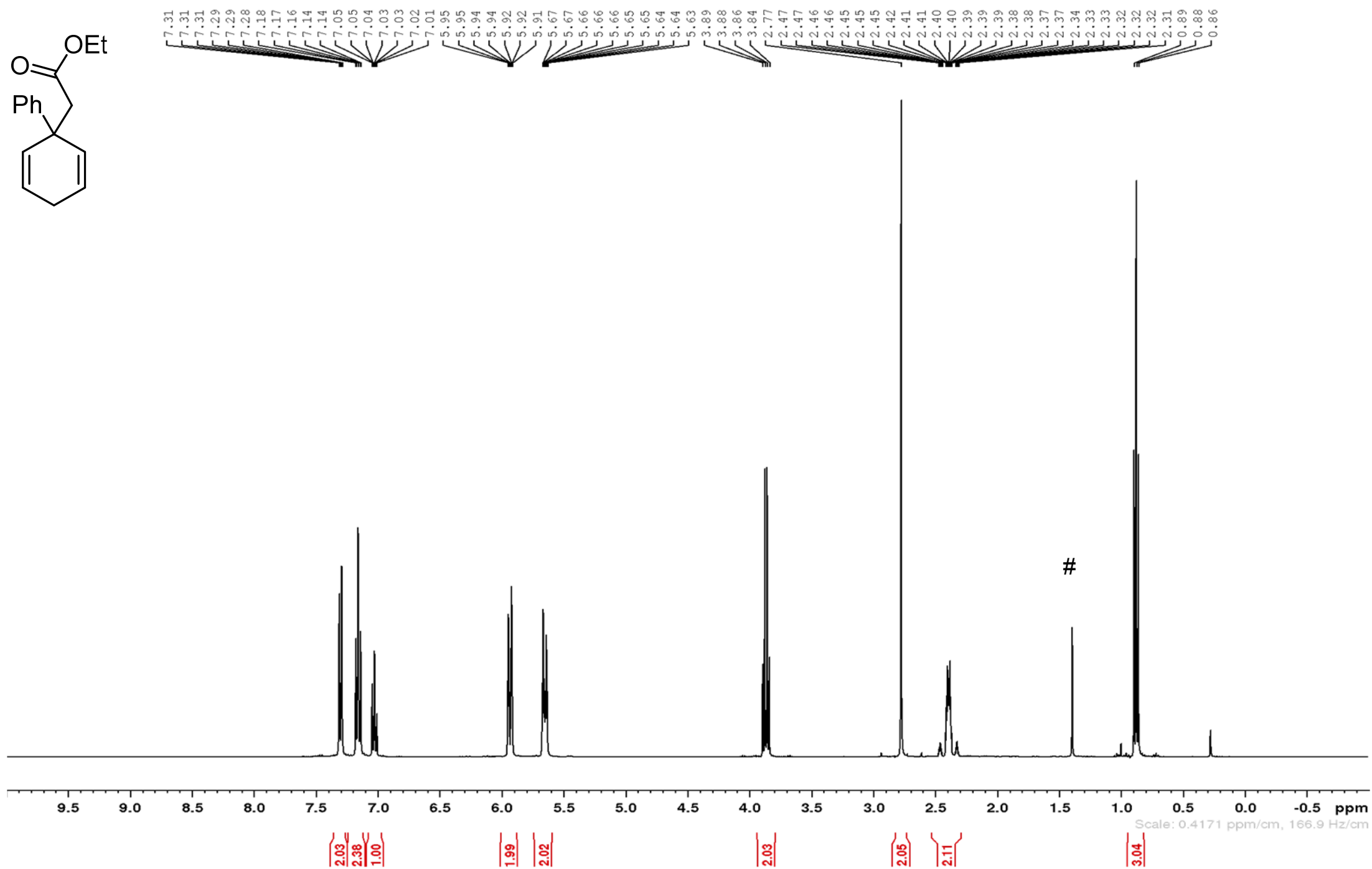
Scheme S25. ${ }^{13} \mathrm{C}\left\{{ }^{1} \mathrm{H}\right\}$ NMR (100 MHz, $\left.\mathrm{C}_{6} \mathrm{D}_{6}, 298 \mathrm{~K}\right)$ of ethyl 2-([1,1'-biphenyl]-1(4H)-yl)acetate (S10)

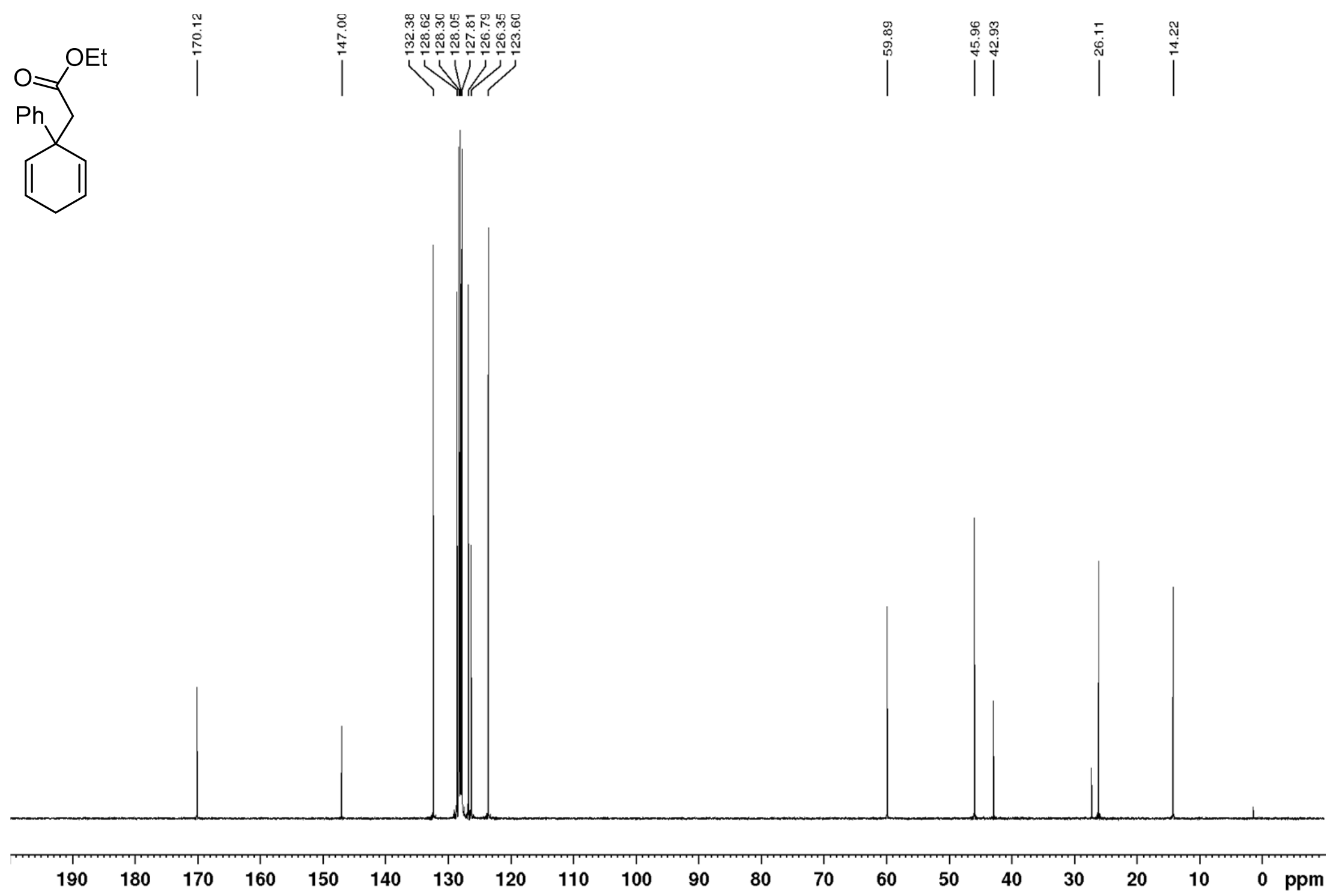


Scheme S26. ${ }^{1} \mathrm{H}$ NMR spectrum $\left(400 \mathrm{MHz}, \mathrm{CDCl}_{3}, 298 \mathrm{~K}\right)$ of 2-([1,1'-biphenyl]-1(4H)-yl)ethan-1-ol (S11)

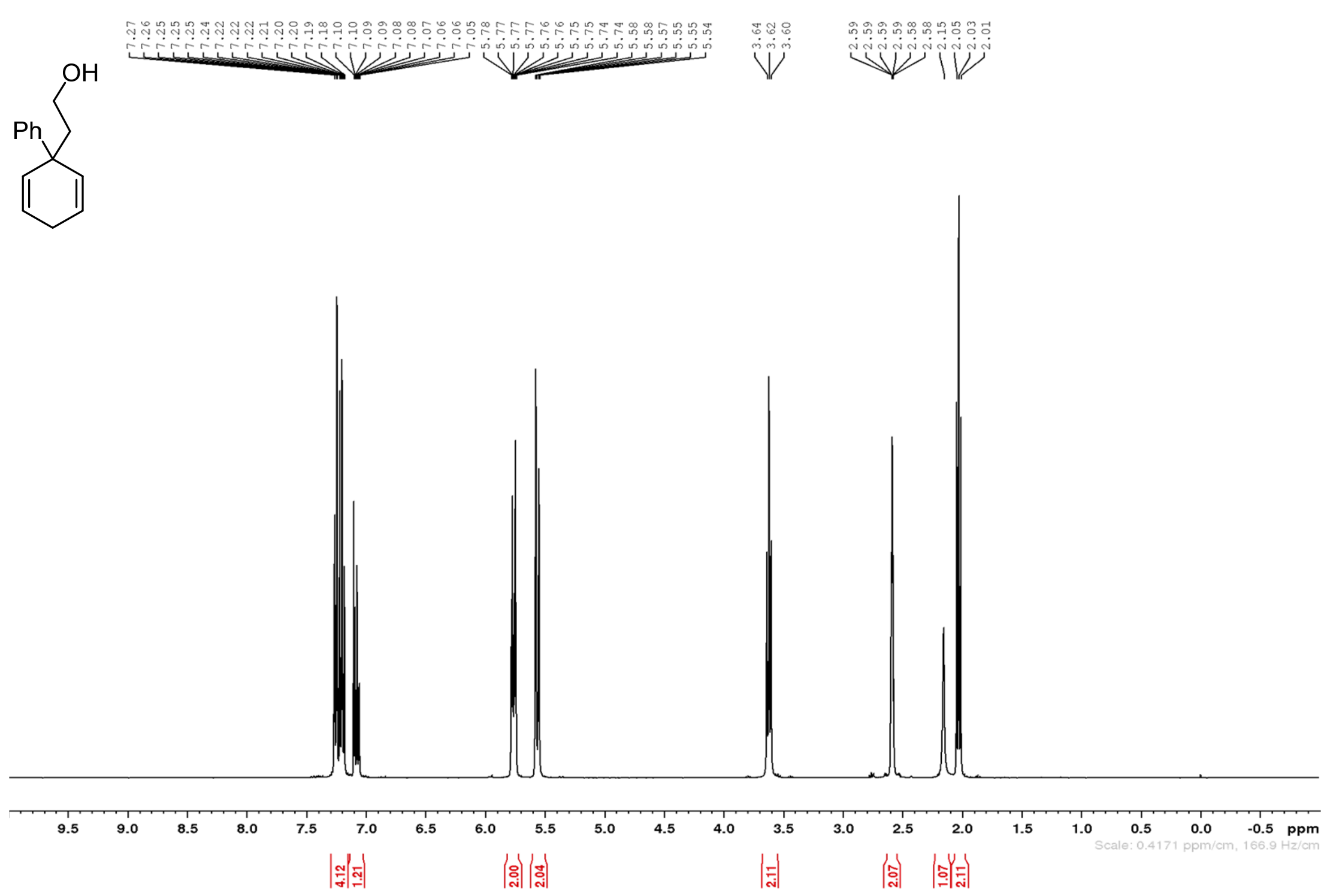


Scheme S27. ${ }^{13} \mathrm{C}\left\{{ }^{1} \mathrm{H}\right\}$ NMR (100 MHz, CDCl, $\left.298 \mathrm{~K}\right)$ of 2-([1,1'-biphenyl]-1(4H)-yl)ethan-1-ol (S11)

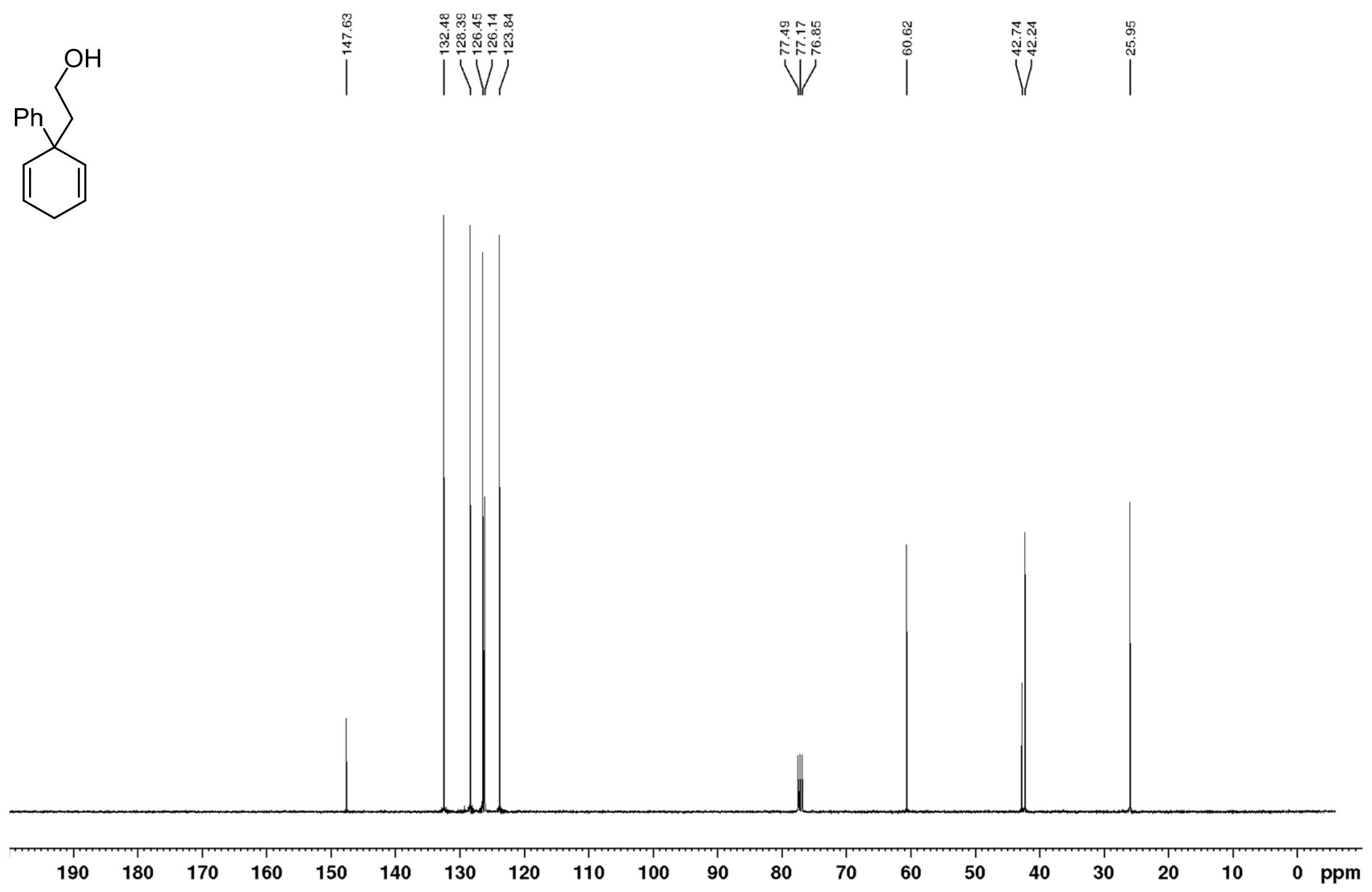


Scheme S28. ${ }^{1} \mathrm{H}$ NMR spectrum (500 MHz, $\mathrm{CD}_{2} \mathrm{Cl}_{2}, 298 \mathrm{~K}$ ) of 1-(2-bromoethyl)-1,4-dihydro-1,1'-biphenyl (3)

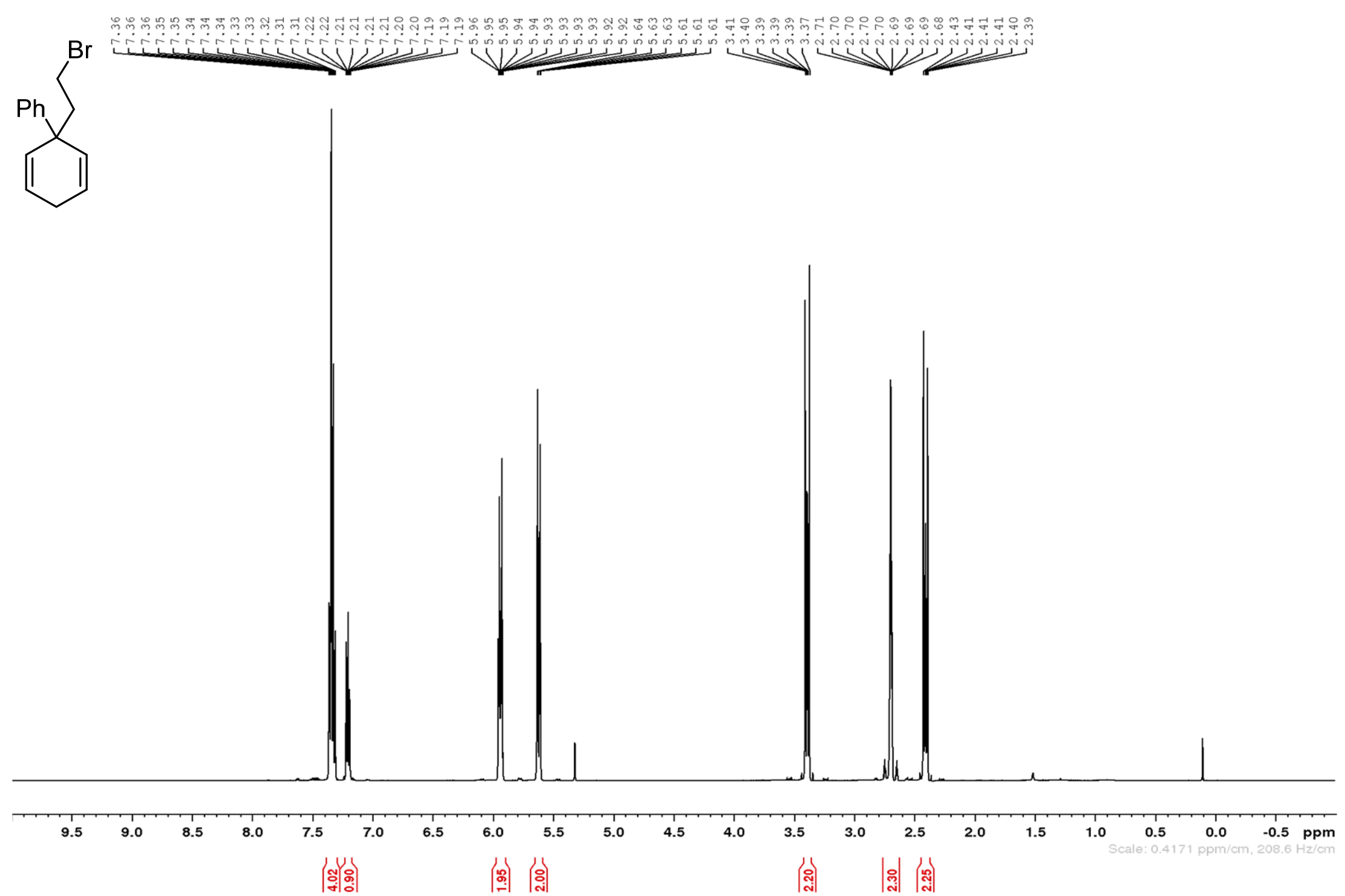


Scheme S29. ${ }^{13} \mathrm{C}\left\{{ }^{1} \mathrm{H}\right\}$ NMR (125 MHz, $\left.\mathrm{CD}_{2} \mathrm{Cl}_{2}, 298 \mathrm{~K}\right)$ of 1-(2-bromoethyl)-1,4-dihydro-1,1'-biphenyl (3)
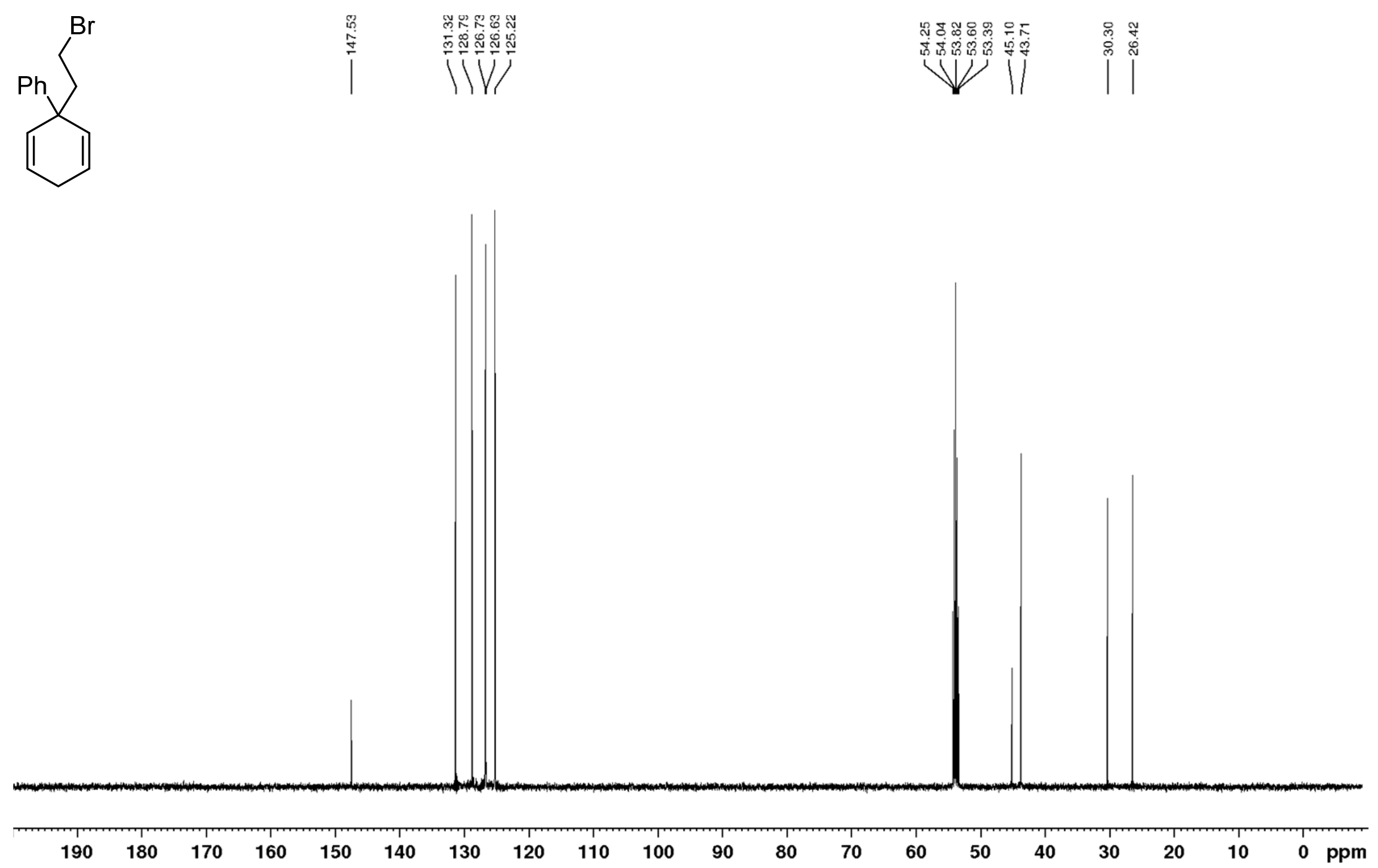
Scheme S30. ${ }^{1} \mathrm{H}$ NMR spectrum $\left(500 \mathrm{MHz}, \mathrm{CD}_{2} \mathrm{Cl}_{2}, 298 \mathrm{~K}\right)$ of 1-(1-bromovinyl)-2-methylbenzene $(5 \mathbf{b})\left(\#=\mathrm{H}_{2} \mathrm{O}\right)$

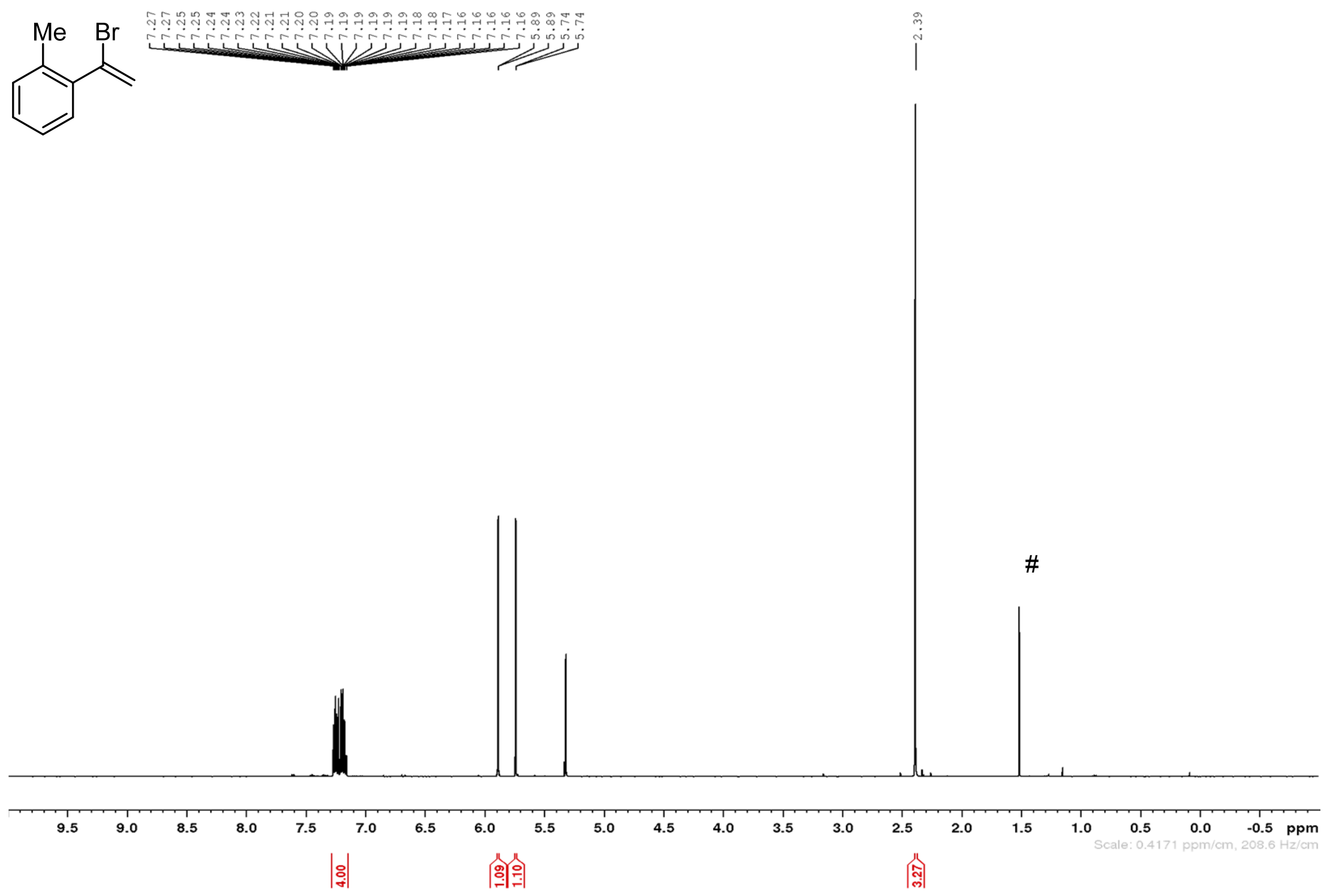


Scheme S31. ${ }^{13} \mathrm{C}\left\{{ }^{1} \mathrm{H}\right\} \mathrm{NMR}\left(125 \mathrm{MHz}, \mathrm{CD}_{2} \mathrm{Cl}_{2}, 298 \mathrm{~K}\right)$ of 1-(1-bromovinyl)-2-methylbenzene (5b)
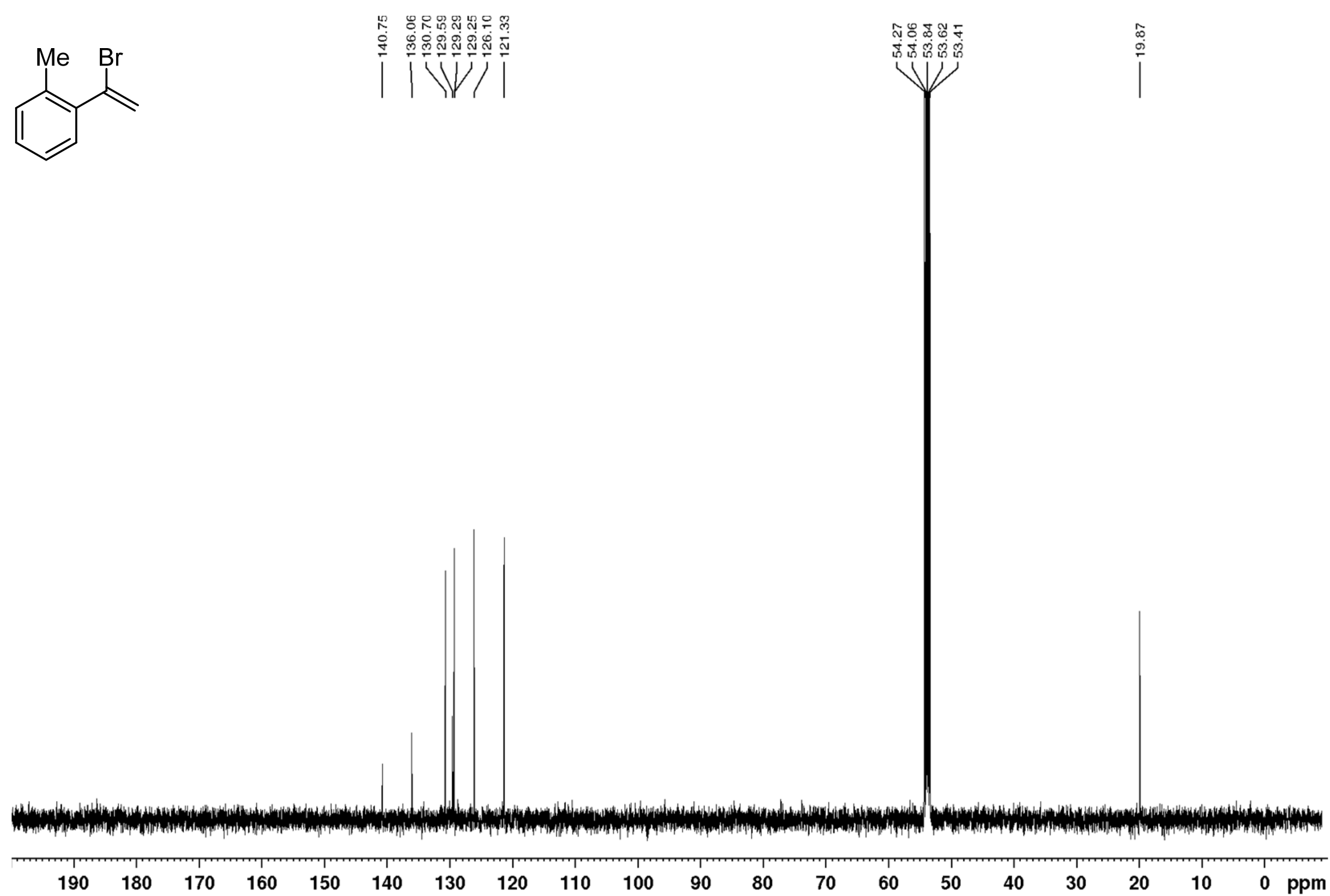
Scheme S32. ${ }^{1} \mathrm{H}$ NMR spectrum (500 MHz, $\mathrm{CD}_{2} \mathrm{Cl}_{2}, 298 \mathrm{~K}$ ) of 2-(4-(1-bromovinyl)phenyl)-4,4,5,5-tetramethyl-1,3,2dioxaborolane $(\mathbf{5 c})\left(\#=\mathrm{H}_{2} \mathrm{O}, \&=\right.$ cyclohexane)
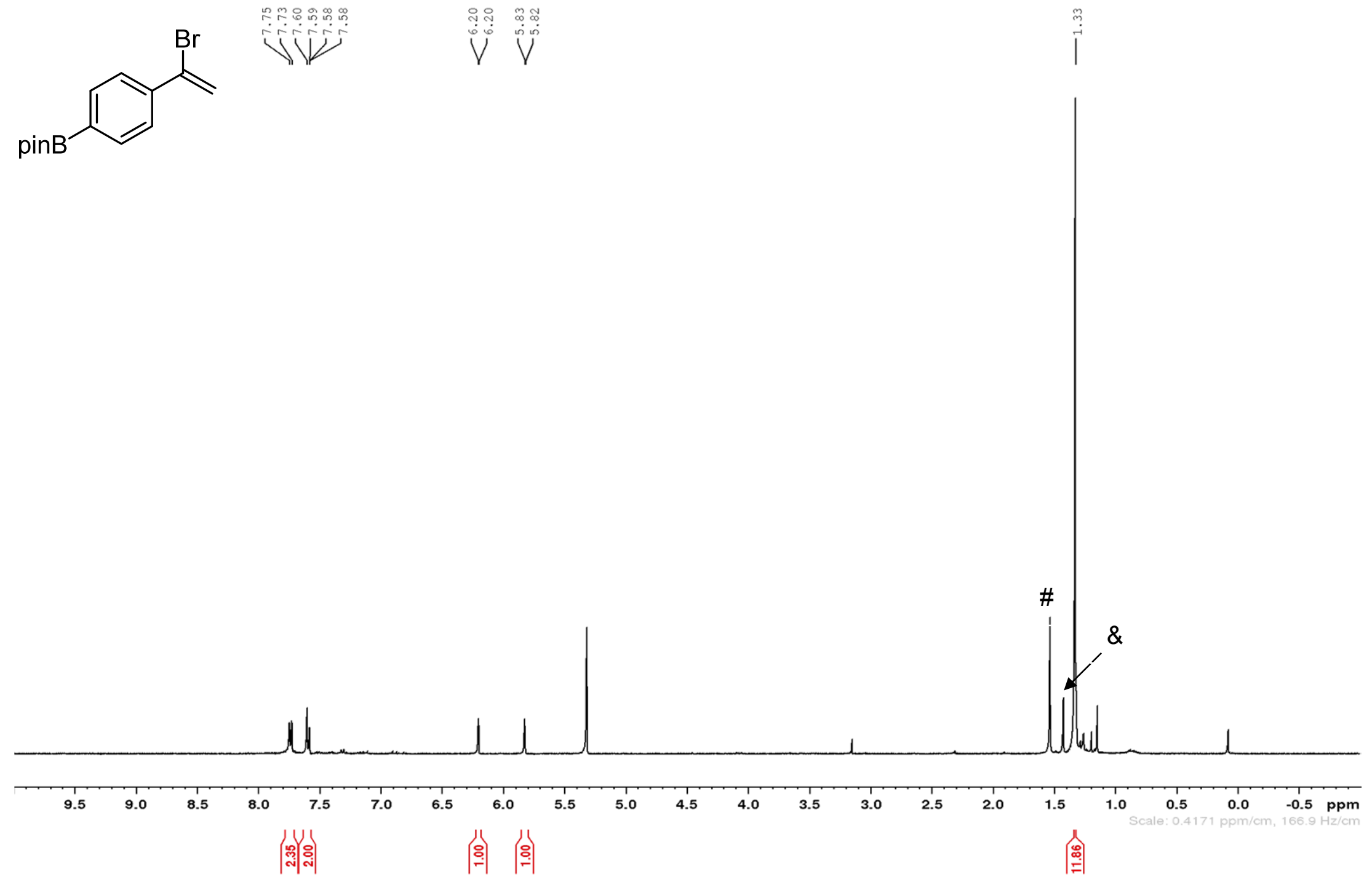
Scheme S33. ${ }^{13} \mathrm{C}\left\{{ }^{1} \mathrm{H}\right\}$ NMR (125 MHz, $\left.\mathrm{CD}_{2} \mathrm{Cl}_{2}, 298 \mathrm{~K}\right)$ of 2-(4-(1-bromovinyl)phenyl)-4,4,5,5-tetramethyl-1,3,2dioxaborolane (5c)
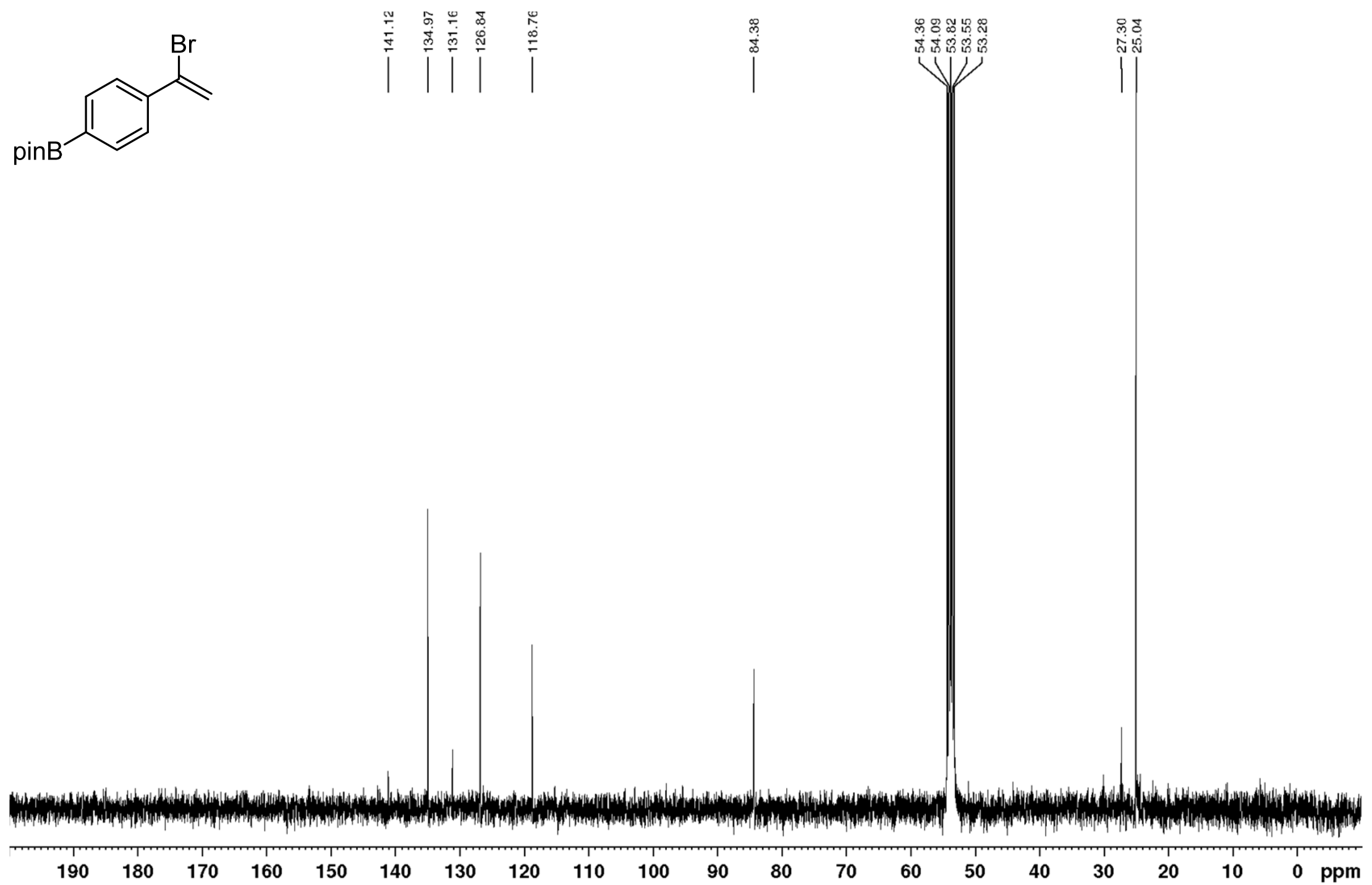
Scheme S34. ${ }^{1} \mathrm{H}$ NMR spectrum (400 MHz, $\left.\mathrm{C}_{6} \mathrm{D}_{6}, 298 \mathrm{~K}\right)$ of (1-bromoprop-1-en-1-yl)benzene $(Z: E=87: 13)(5 \mathrm{~d})$

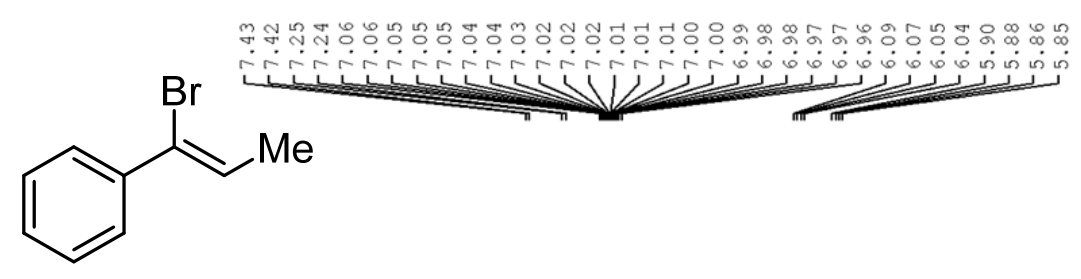

z Z

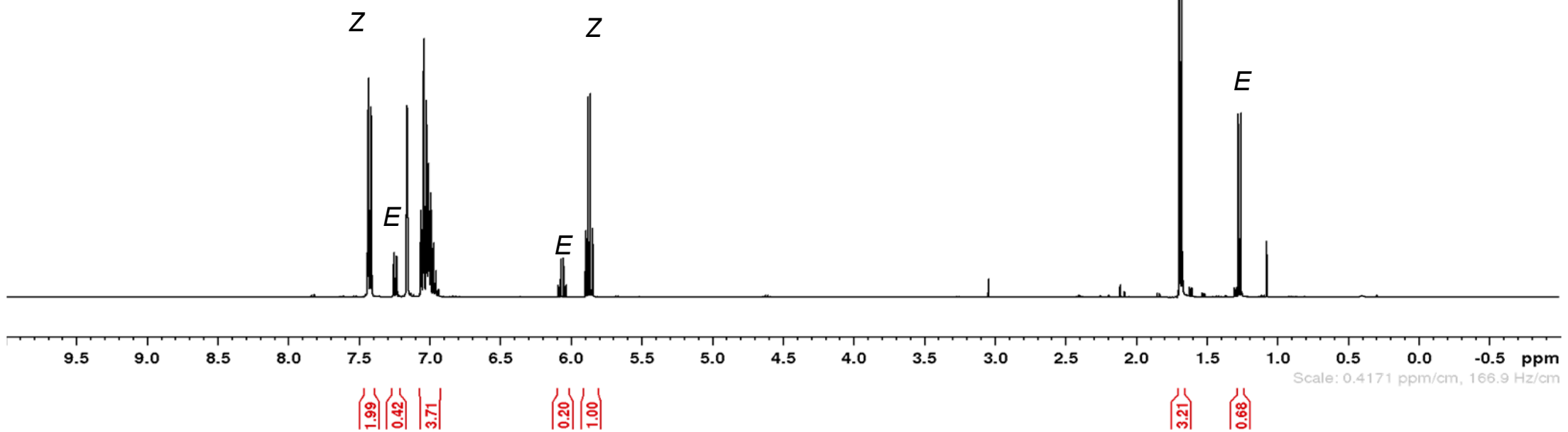


Scheme S35. ${ }^{13} \mathrm{C}\left\{{ }^{1} \mathrm{H}\right\}$ NMR (100 MHz, $\left.\mathrm{C}_{6} \mathrm{D}_{6}, 298 \mathrm{~K}\right)$ of (1-bromoprop-1-en-1-yl)benzene (Z:E = 87:13) (5d)

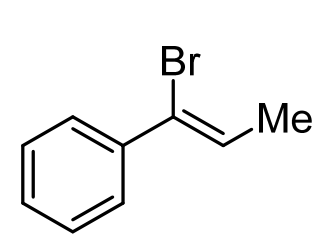

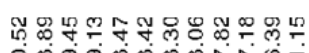

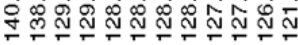

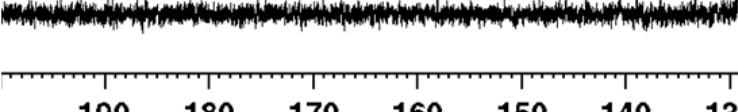


Scheme S36. ${ }^{1} \mathrm{H}$ NMR spectrum (500 MHz, $\mathrm{CD}_{2} \mathrm{Cl}_{2}, 298 \mathrm{~K}$ ) of 4-(1-bromovinyl)benzoic acid (5f)

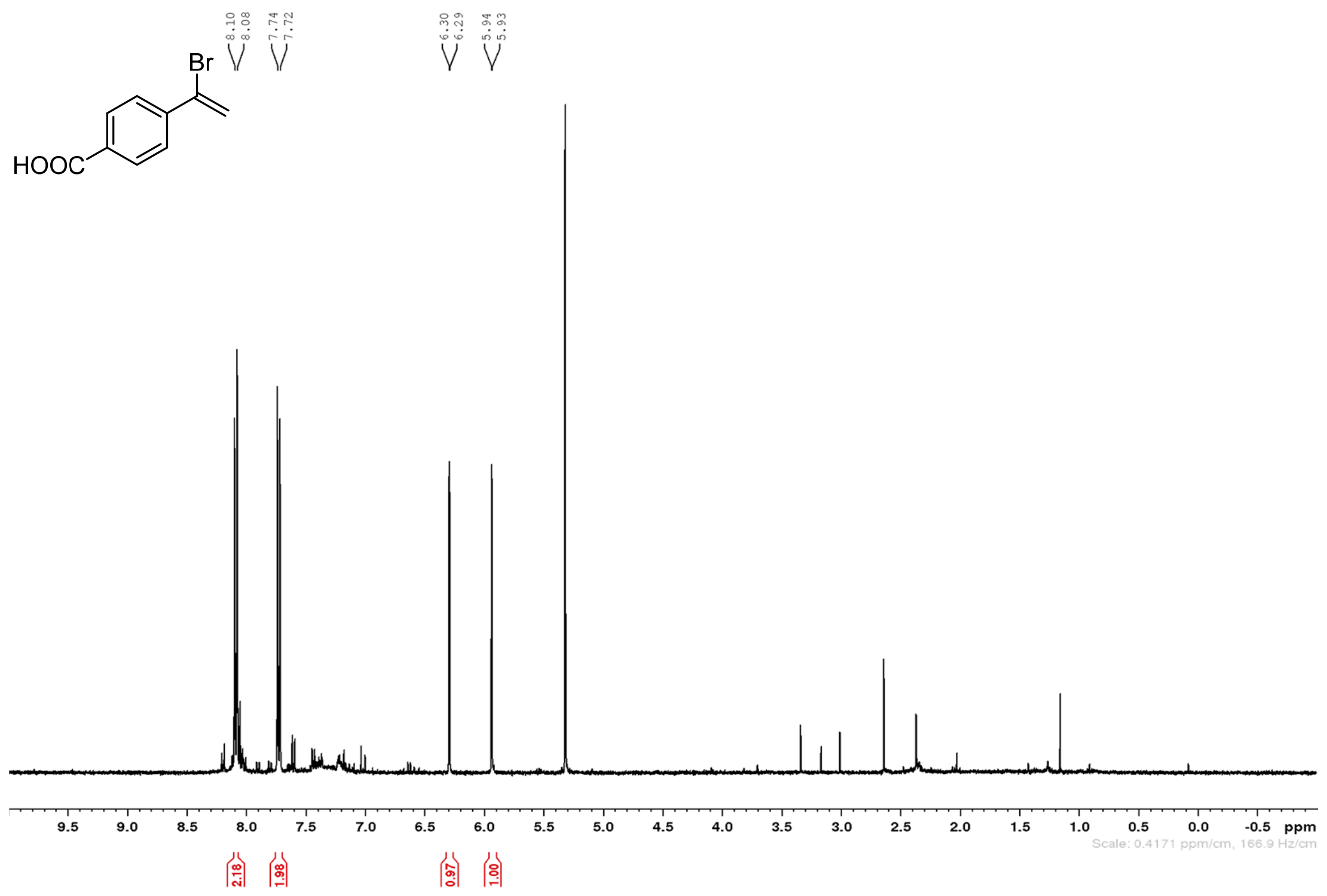


Scheme S37. ${ }^{13} \mathrm{C}\left\{{ }^{1} \mathrm{H}\right\} \mathrm{NMR}\left(125 \mathrm{MHz}, \mathrm{CD}_{2} \mathrm{Cl}_{2}, 298 \mathrm{~K}\right)$ of 4-(1-bromovinyl)benzoic acid (5f)<smiles>C=C(Br)c1ccc(C(=O)O)cc1</smiles>
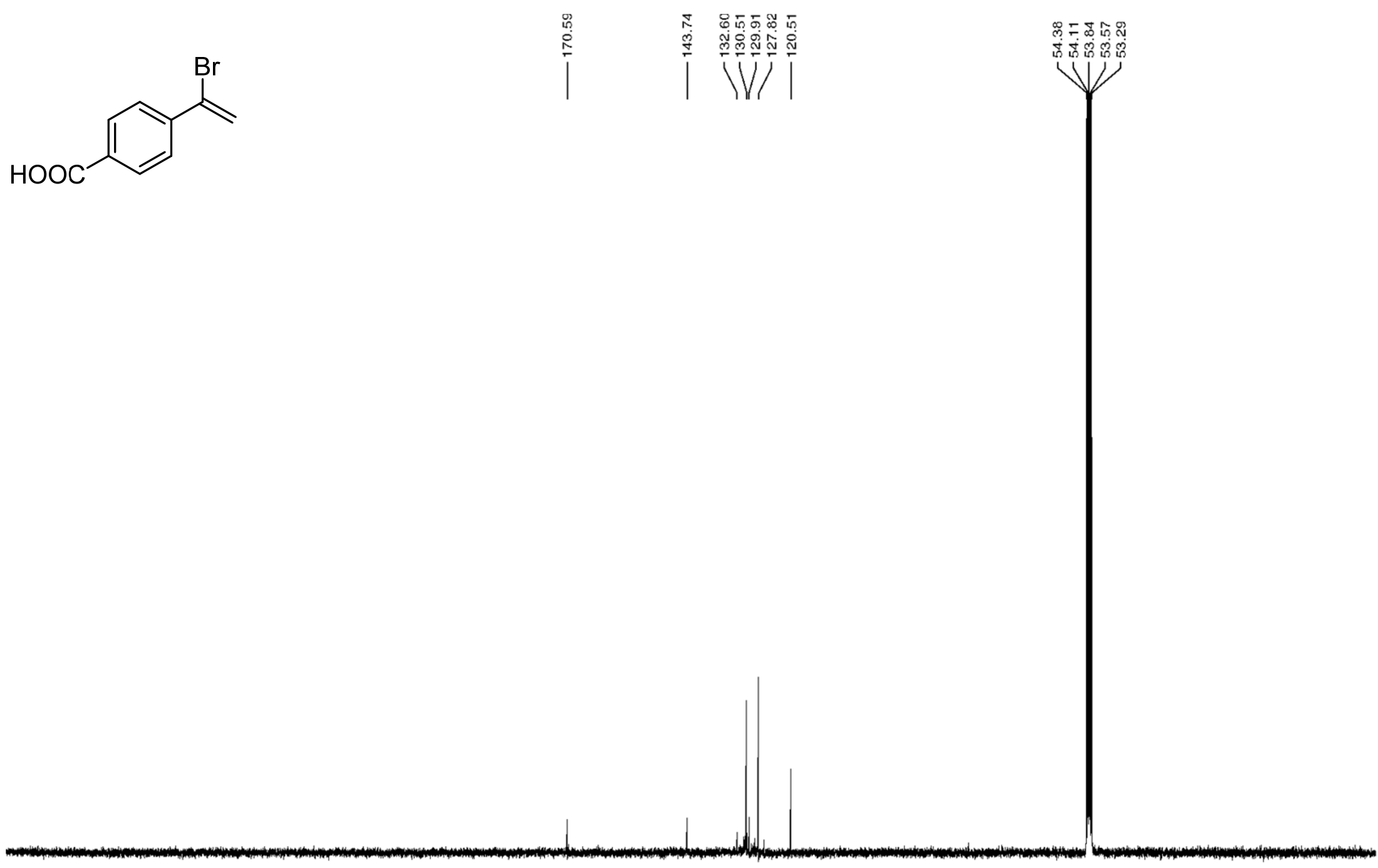
Scheme S38. ${ }^{1} \mathrm{H}$ NMR spectrum $\left(500 \mathrm{MHz}, \mathrm{CD}_{2} \mathrm{Cl}_{2}, 298 \mathrm{~K}\right)$ of 3-bromo-1,3-diphenylprop-2-en-1-one $(Z: E>99: 1)(7 \mathrm{a})$

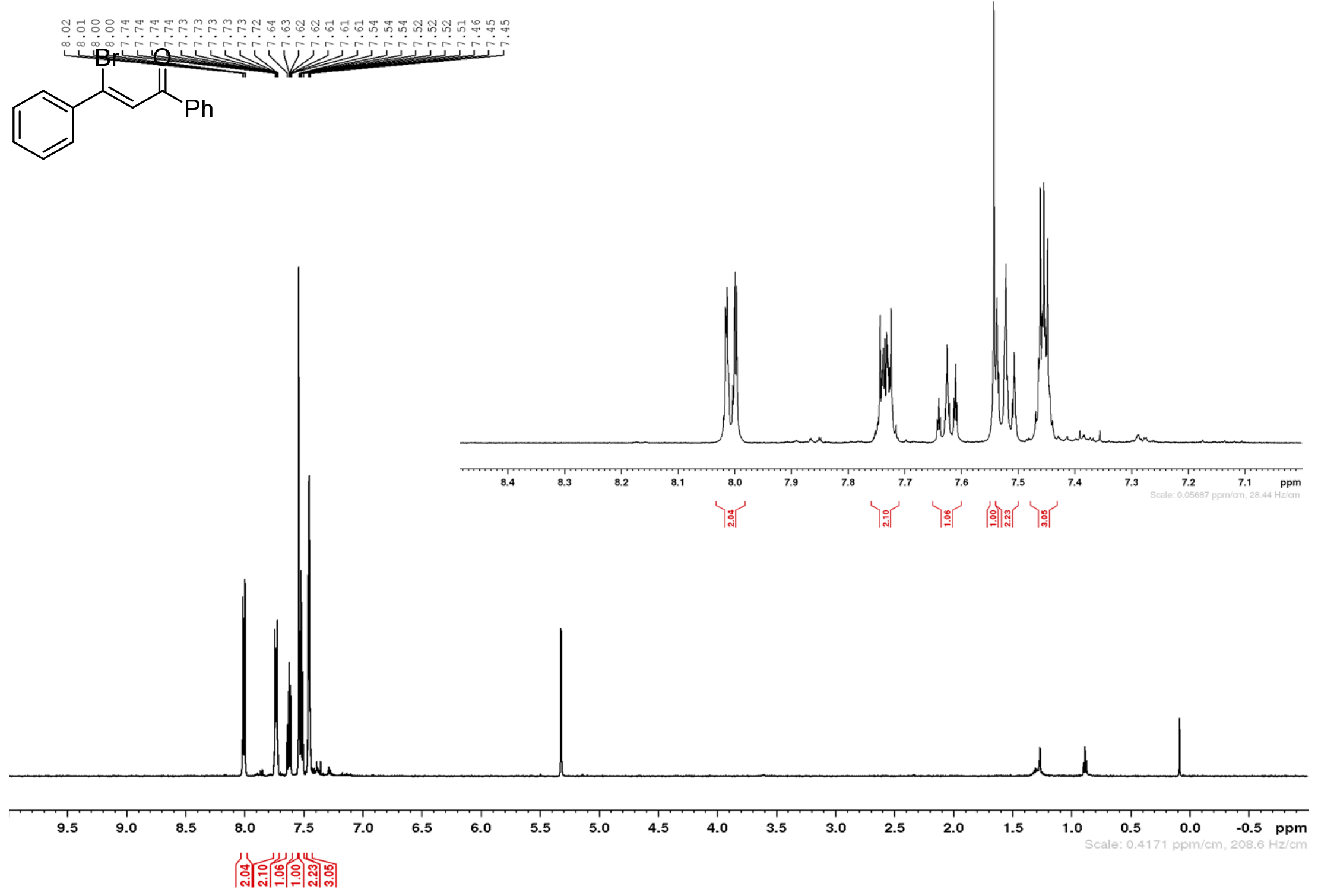


Scheme S39. ${ }^{13} \mathrm{C}\left\{{ }^{1} \mathrm{H}\right\}$ NMR (125 MHz, $\left.\mathrm{CD}_{2} \mathrm{Cl}_{2}, 298 \mathrm{~K}\right)$ of 3-bromo-1,3-diphenylprop-2-en-1-one $(Z: E>99: 1)(7 \mathbf{a})$
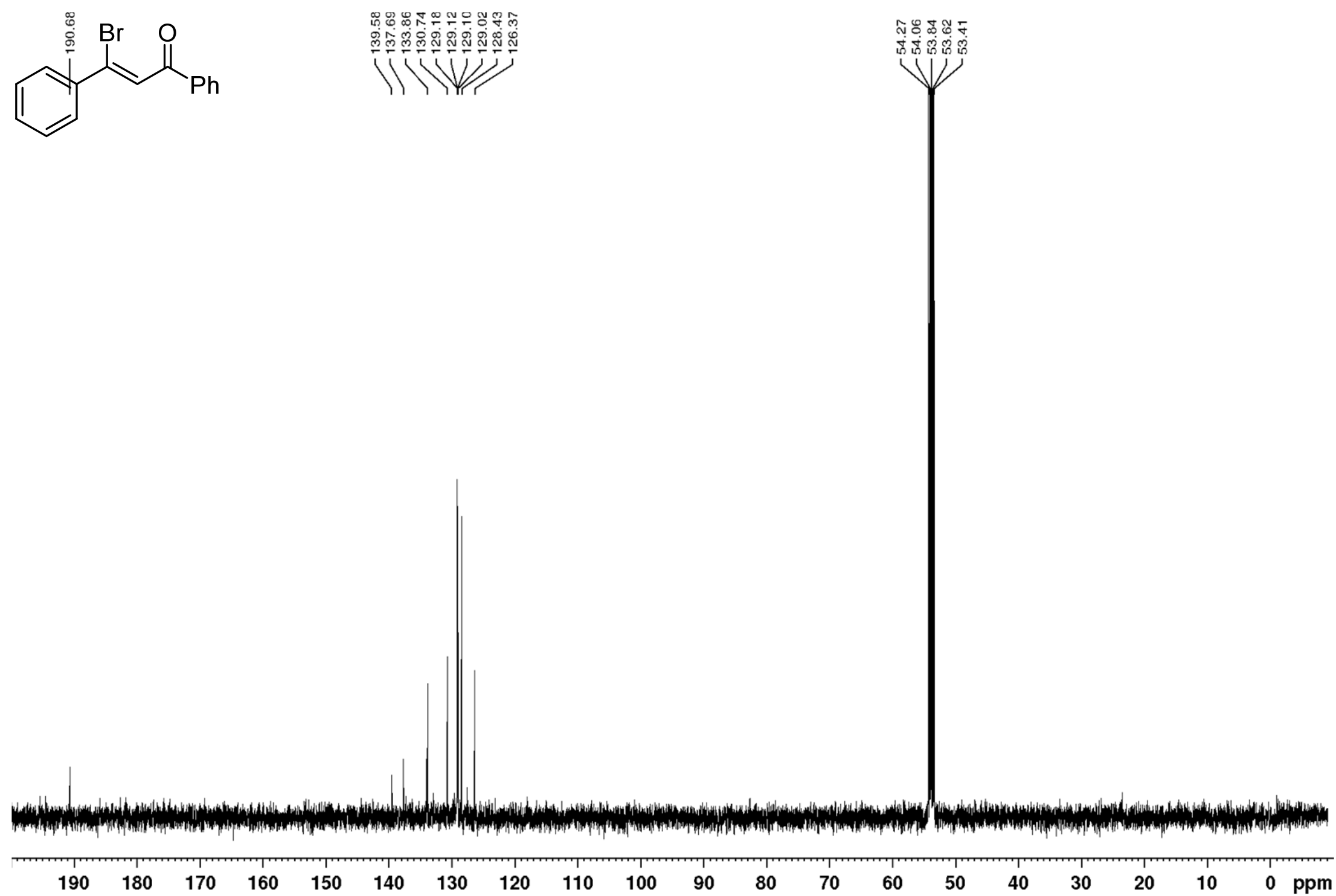
Scheme S40. ${ }^{1} \mathrm{H}$ NMR spectrum $\left(500 \mathrm{MHz}, \mathrm{CD}_{2} \mathrm{Cl}_{2}, 298 \mathrm{~K}\right)$ of 3-bromo-3-phenylacrylic acid $(Z: E=79: 21)(7 \mathbf{b})$
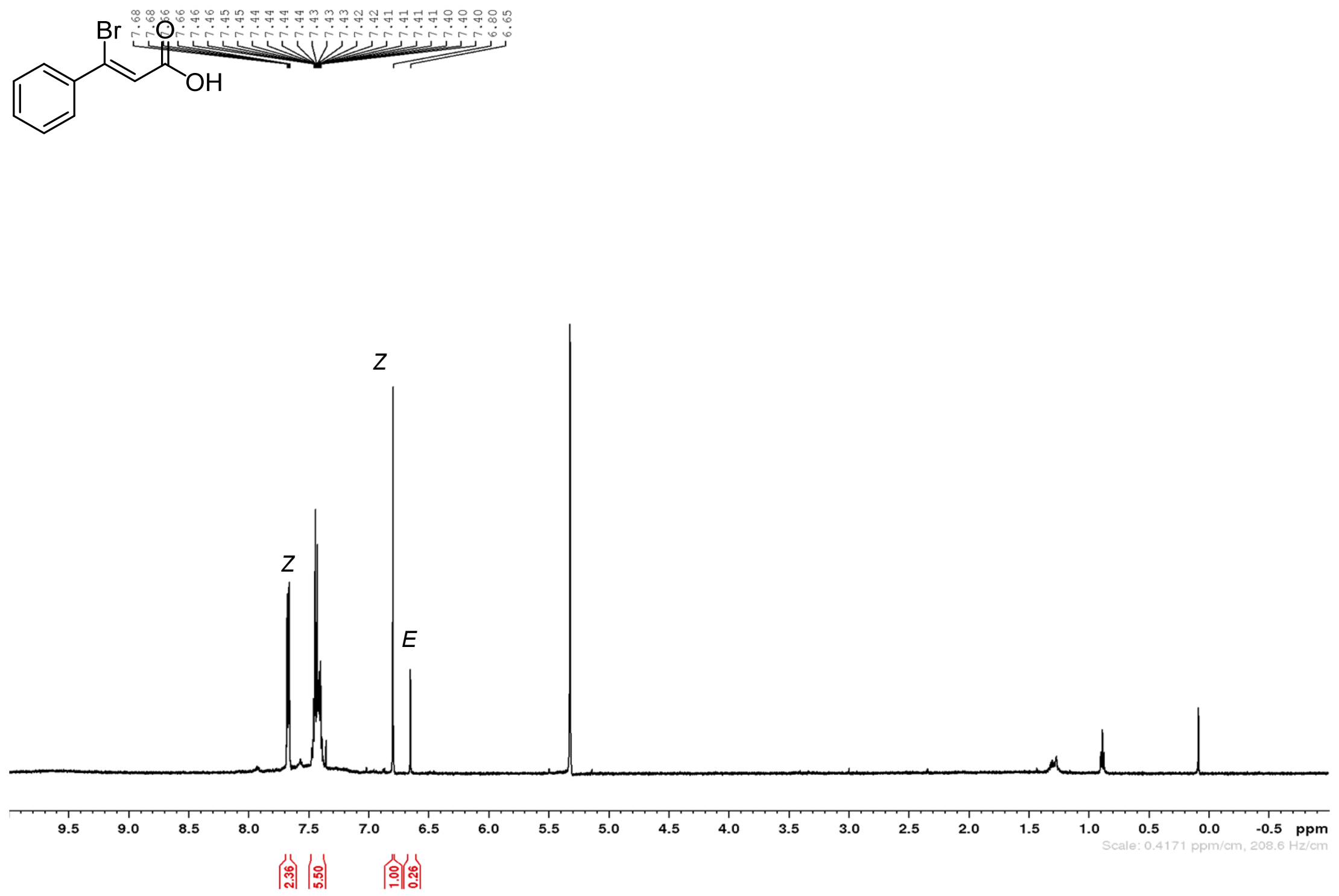
Scheme S41. ${ }^{13} \mathrm{C}\left\{{ }^{1} \mathrm{H}\right\}$ NMR (125 MHz, $\left.\mathrm{CD}_{2} \mathrm{Cl}_{2}, 298 \mathrm{~K}\right)$ of 3-bromo-3-phenylacrylic acid $(Z: E=79: 21)(7 \mathbf{b})$
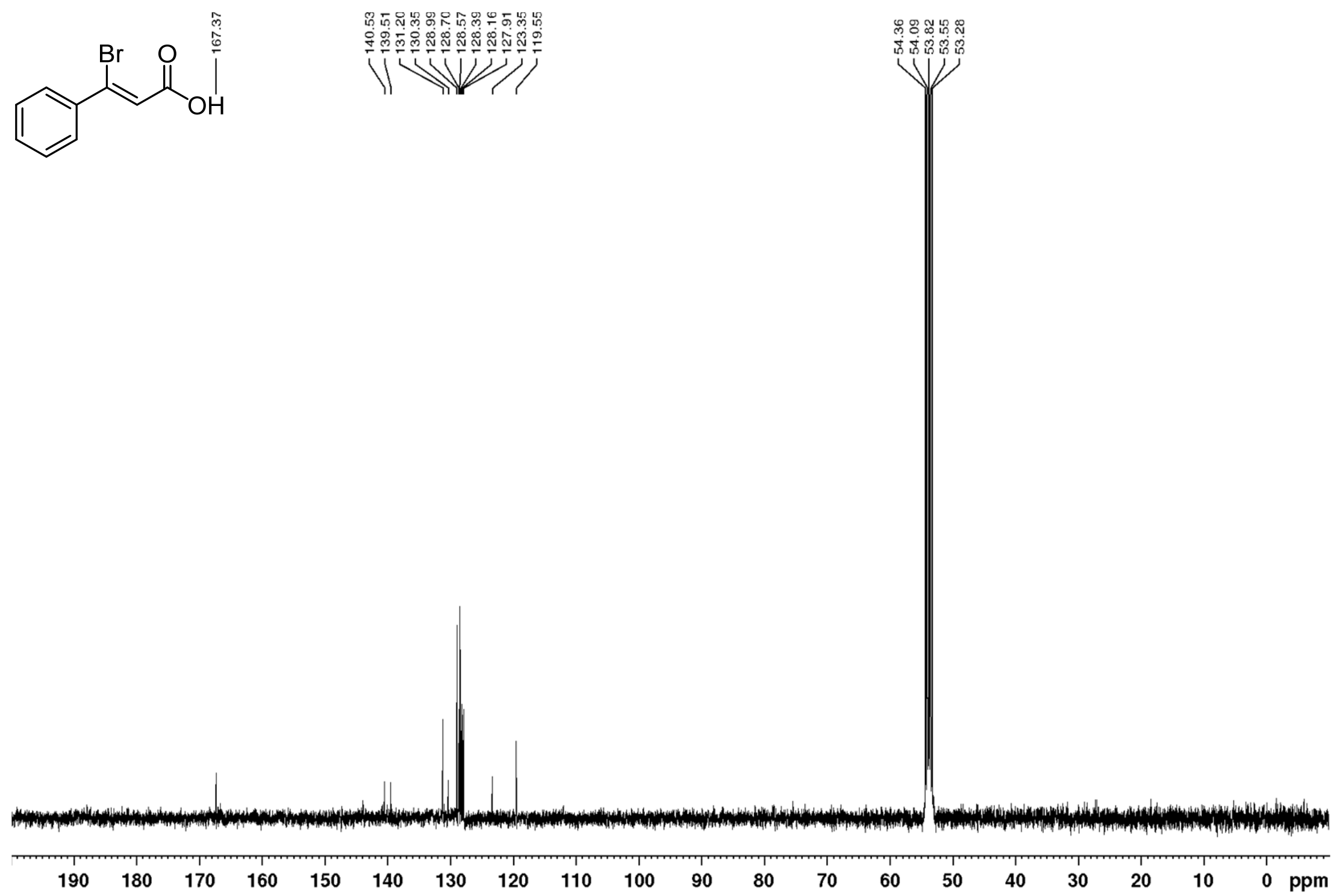
Scheme S42. ${ }^{1} \mathrm{H}$ NMR spectrum $\left(500 \mathrm{MHz}, \mathrm{C}_{6} \mathrm{D}_{6}, 298 \mathrm{~K}\right)$ of (Z)-3-iodo- $N, N$-dimethyl-3-phenylacrylamide (Z-7c)

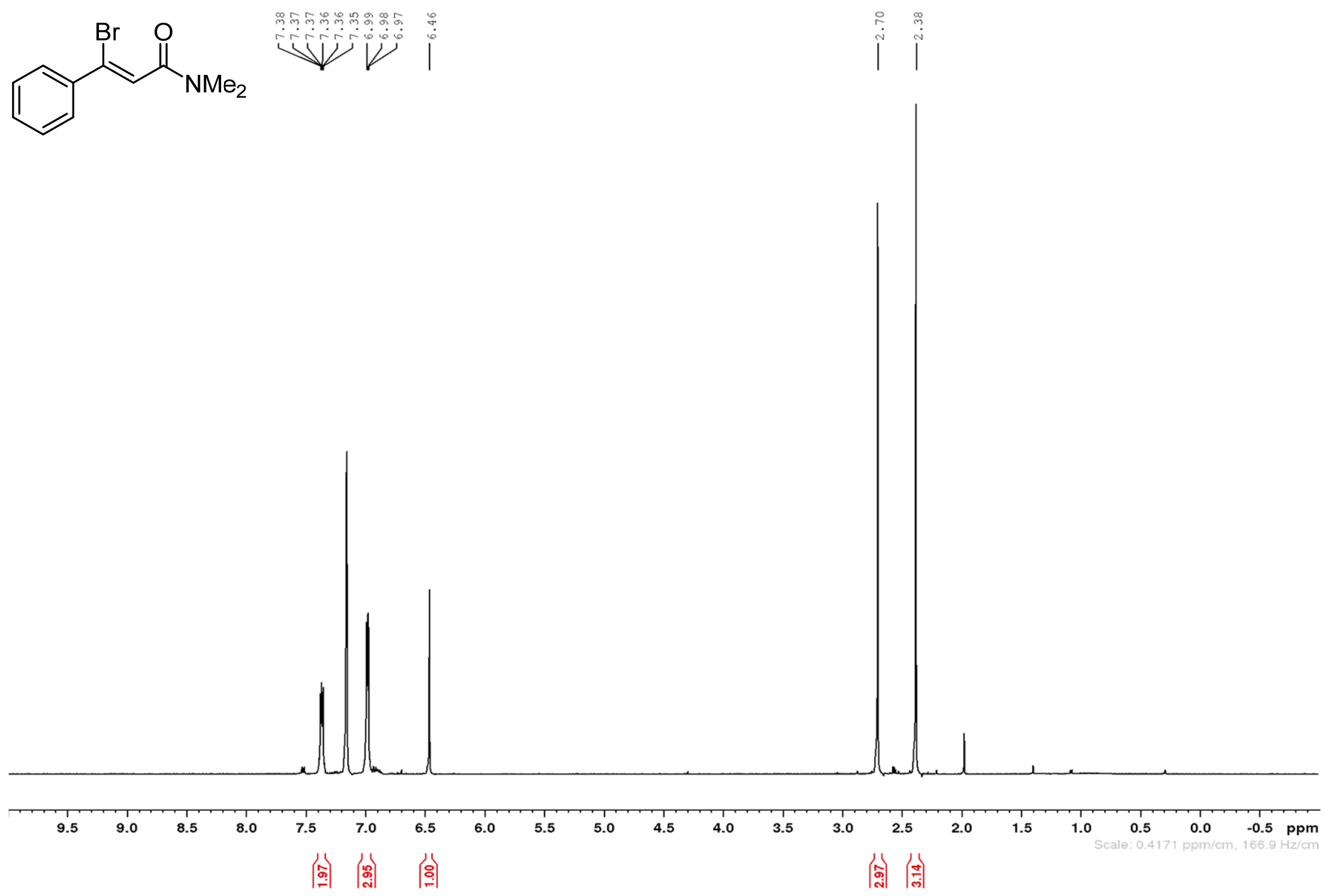


Scheme S43. ${ }^{13} \mathrm{C}\left\{{ }^{1} \mathrm{H}\right\}$ NMR (125 MHz, $\left.\mathrm{C}_{6} \mathrm{D}_{6}, 298 \mathrm{~K}\right)$ of (Z)-3-iodo- $N, N$-dimethyl-3-phenylacrylamide (Z-7c)

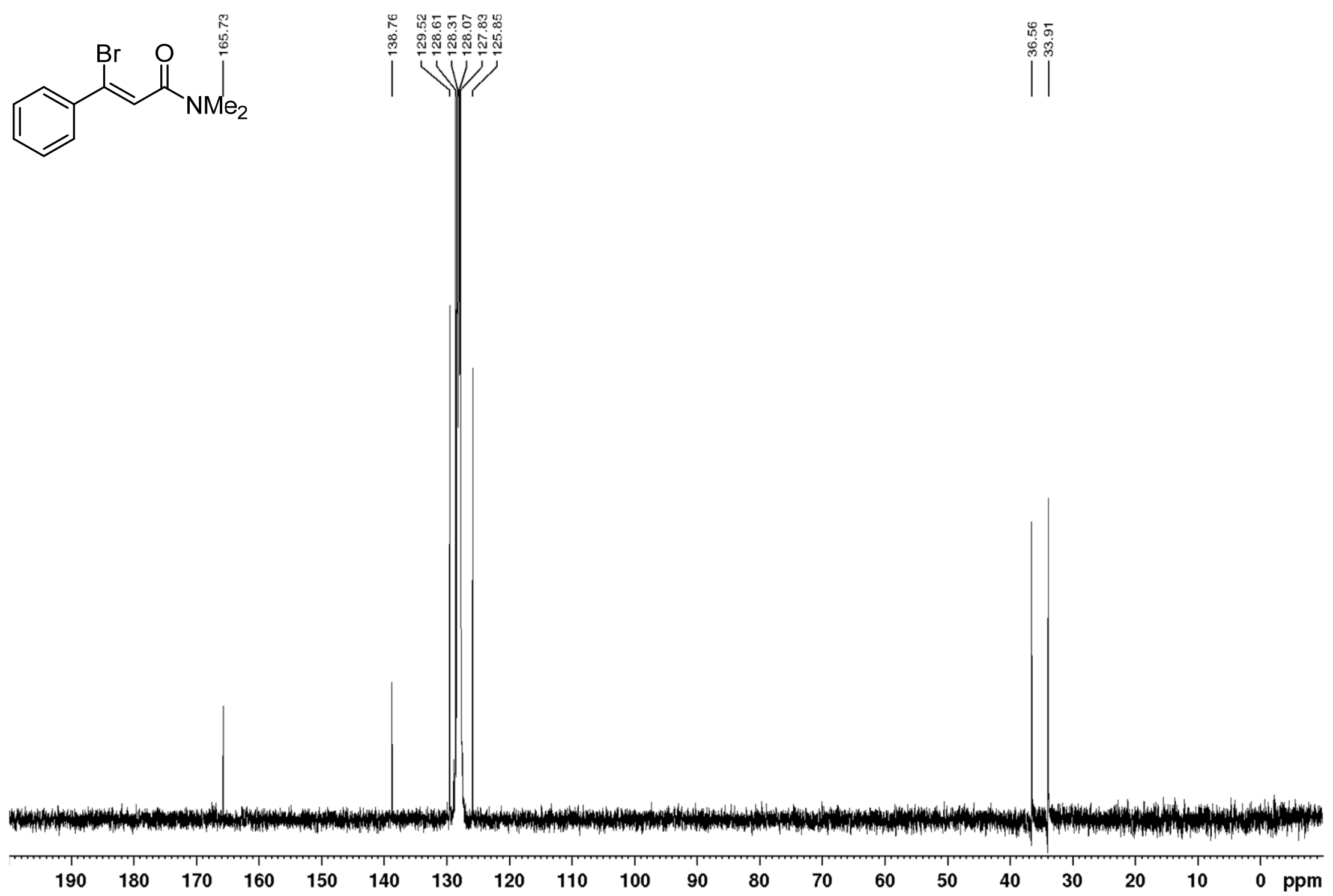


Scheme S44. ${ }^{1} \mathrm{H}$ NMR spectrum $\left(500 \mathrm{MHz}, \mathrm{C}_{6} \mathrm{D}_{6}, 298 \mathrm{~K}\right)$ of $(E)-3$-iodo- $N, N$-dimethyl-3-phenylacrylamide $(E-7 \mathrm{c})\left(\#=\mathrm{H}_{2} \mathrm{O}\right.$, $\&=N, N$-dimethyl-3-phenylpropiolamide (6c))
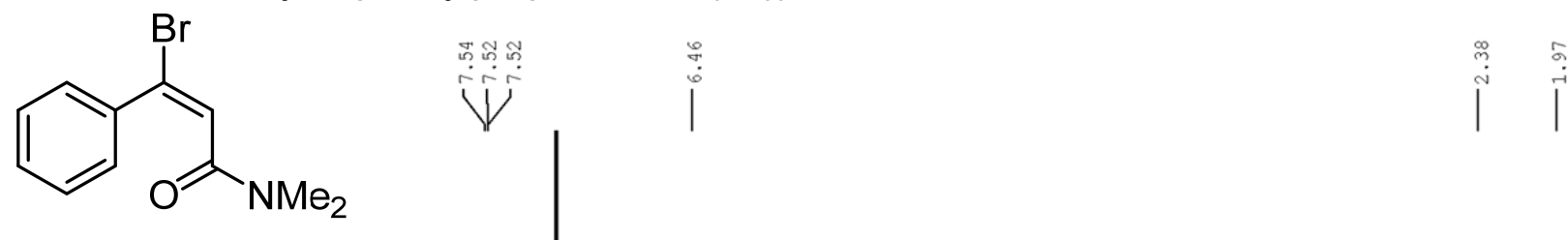

$\&$

\#

$\&$

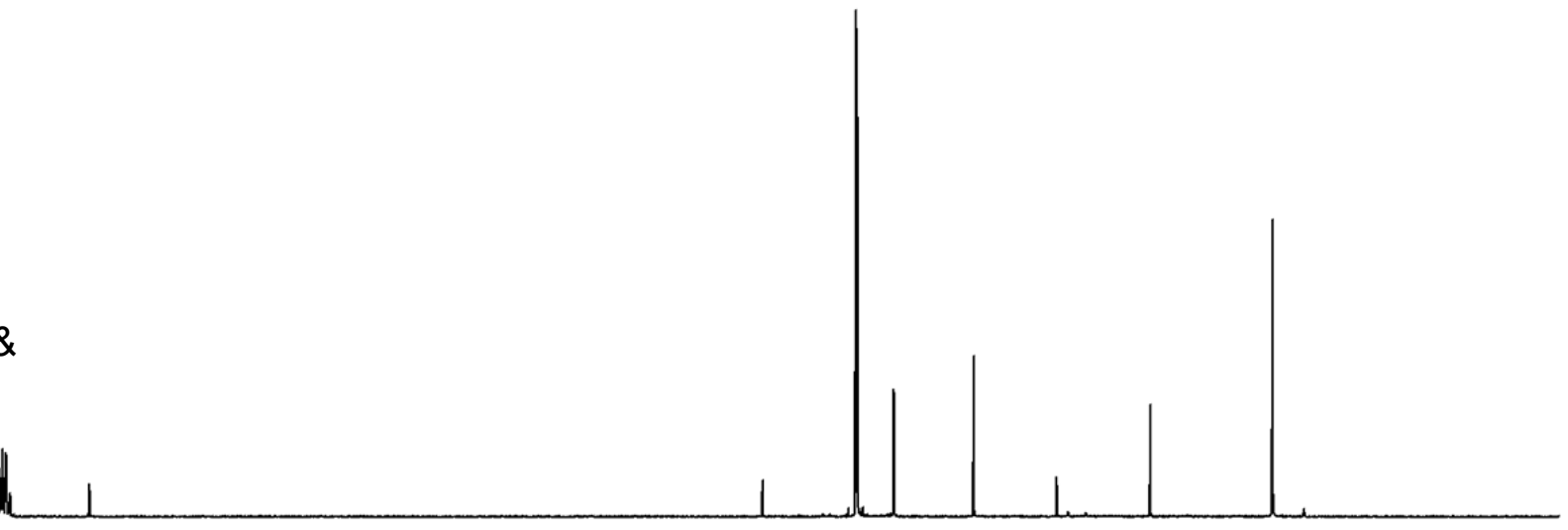

$\begin{array}{llll}9.5 & 9.0 & 8.5 & 8.0\end{array}$

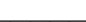

6.

亩

㖉 馬 
Scheme S45. ${ }^{1} \mathrm{H}$ NMR spectrum (400 MHz, $\mathrm{C}_{6} \mathrm{D}_{6}, 298 \mathrm{~K}$ ) of (Z)-3-bromo-3-(4-chlorophenyl)-N,N-dimethylacrylamide (Z7d)<smiles>CNC(=O)C=C(Br)c1ccc(Cl)cc1</smiles>
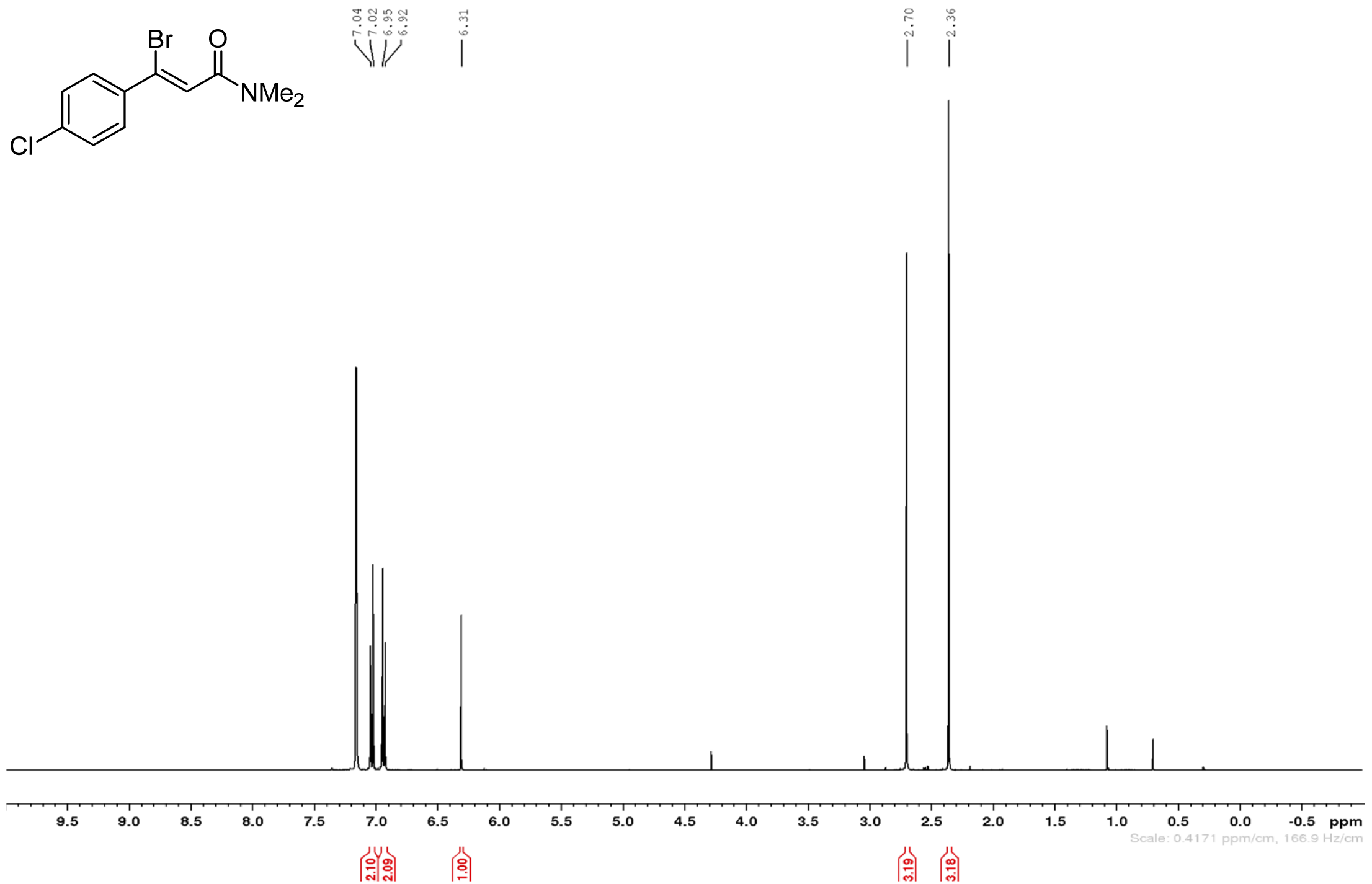
Scheme S46. ${ }^{13} \mathrm{C}\left\{{ }^{1} \mathrm{H}\right\}$ NMR (100 MHz, $\left.\mathrm{C}_{6} \mathrm{D}_{6}, 298 \mathrm{~K}\right)$ of (Z)-3-bromo-3-(4-chlorophenyl)-N,N-dimethylacrylamide (Z-7d)

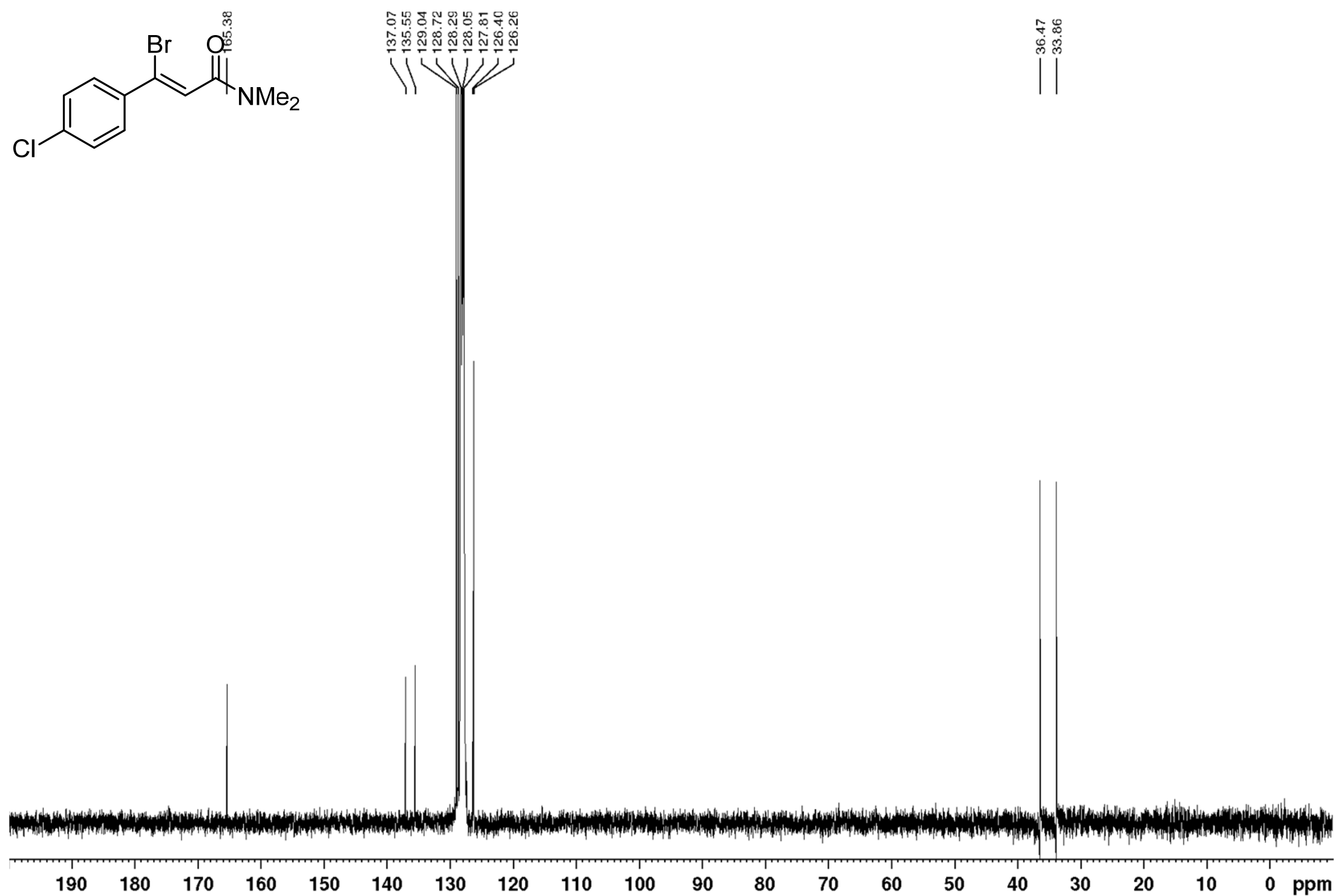


Scheme S47. ${ }^{1} \mathrm{H}$ NMR spectrum (400 MHz, $\left.\mathrm{C}_{6} \mathrm{D}_{6}, 298 \mathrm{~K}\right)$ of $(E)$-3-bromo-3-(4-chlorophenyl)- $N, N$-dimethylacrylamide $(E-$ 7d) (\& = 3-(4-chlorophenyl)-N,N-dimethylpropiolamide (6d))

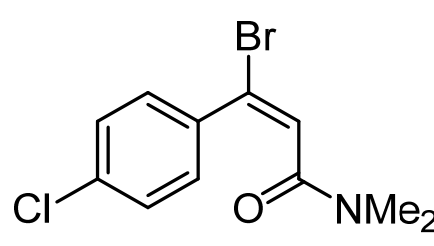

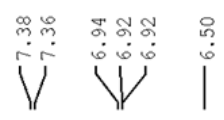
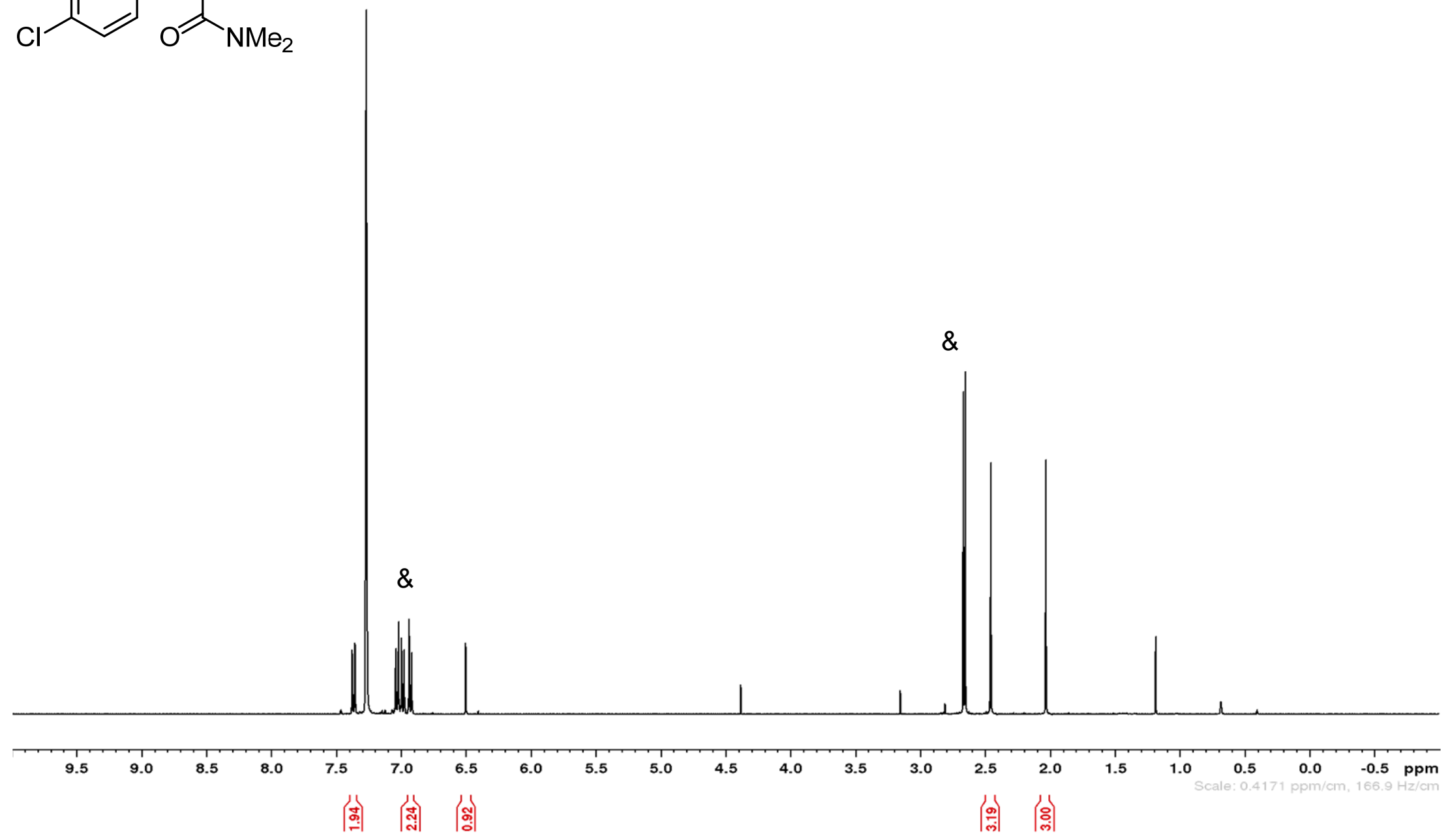
Scheme S48. ${ }^{13} \mathrm{C}\left\{{ }^{1} \mathrm{H}\right\} \mathrm{NMR}\left(100 \mathrm{MHz}, \mathrm{C}_{6} \mathrm{D}_{6}, 298 \mathrm{~K}\right)$ of $(E)-3$-bromo-3-(4-chlorophenyl)- $N, N$-dimethylacrylamide $(E-7 \mathrm{~d})$
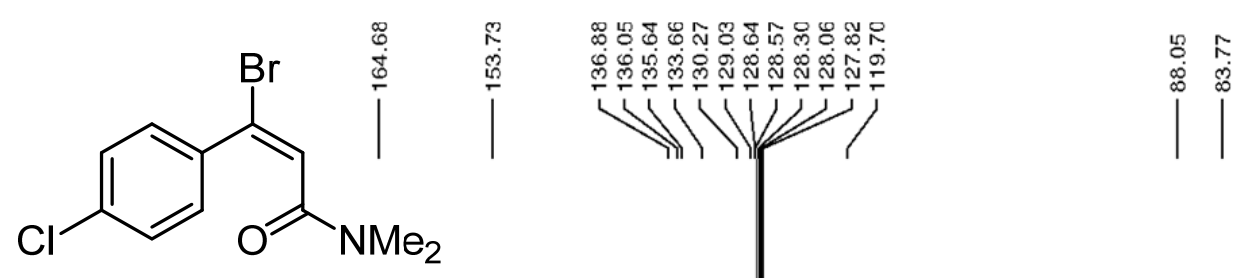

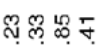

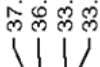

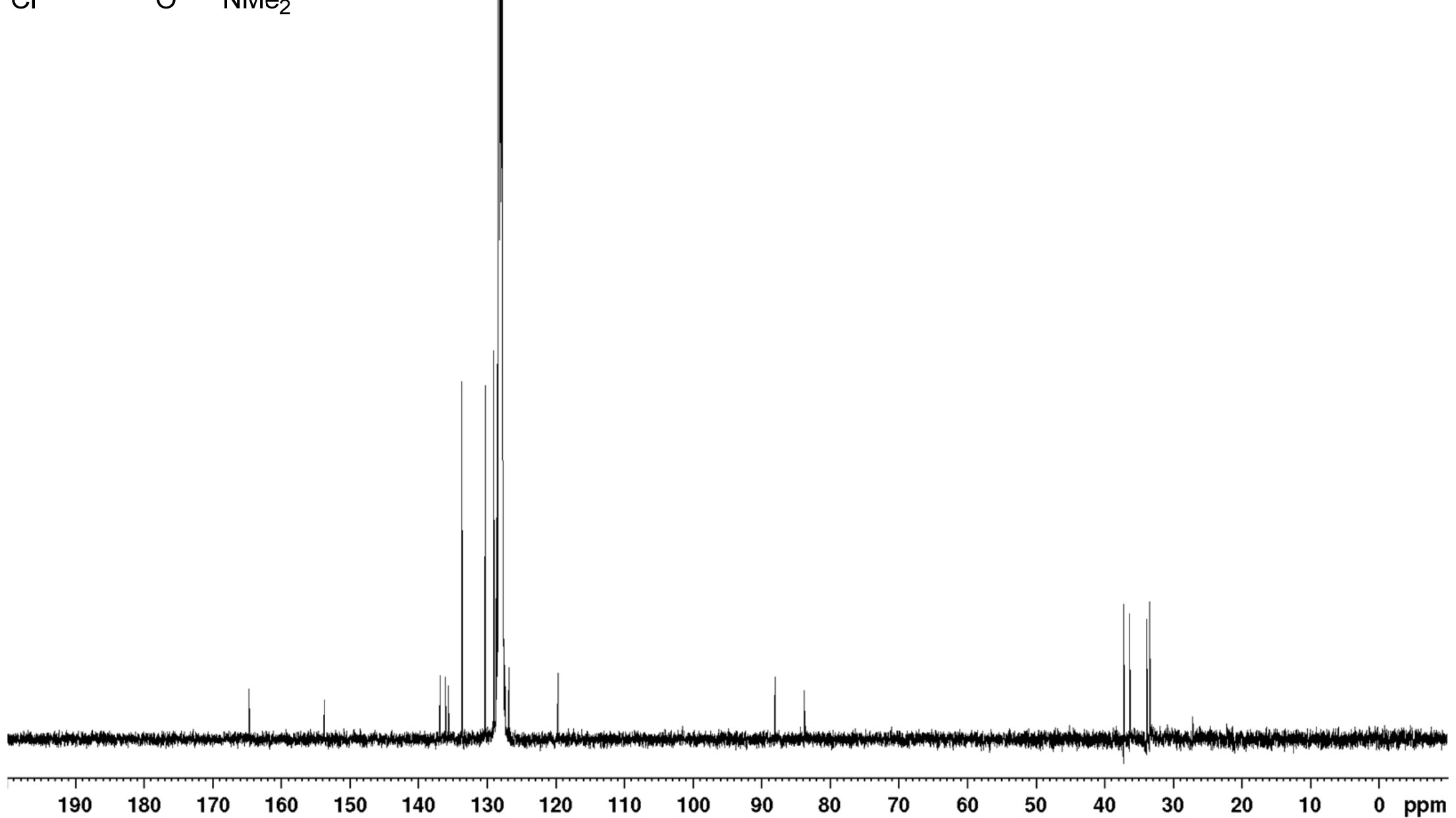


Scheme S49. ${ }^{1} \mathrm{H}$ NMR spectrum (400 MHz, $\left.\quad \mathrm{C}_{6} \mathrm{D}_{6}, 298 \mathrm{~K}\right)$ of (Z)-3-bromo-N,N-dimethyl-3-(4-(trifluoromethyl) phenyl)acrylamide (Z-7e)
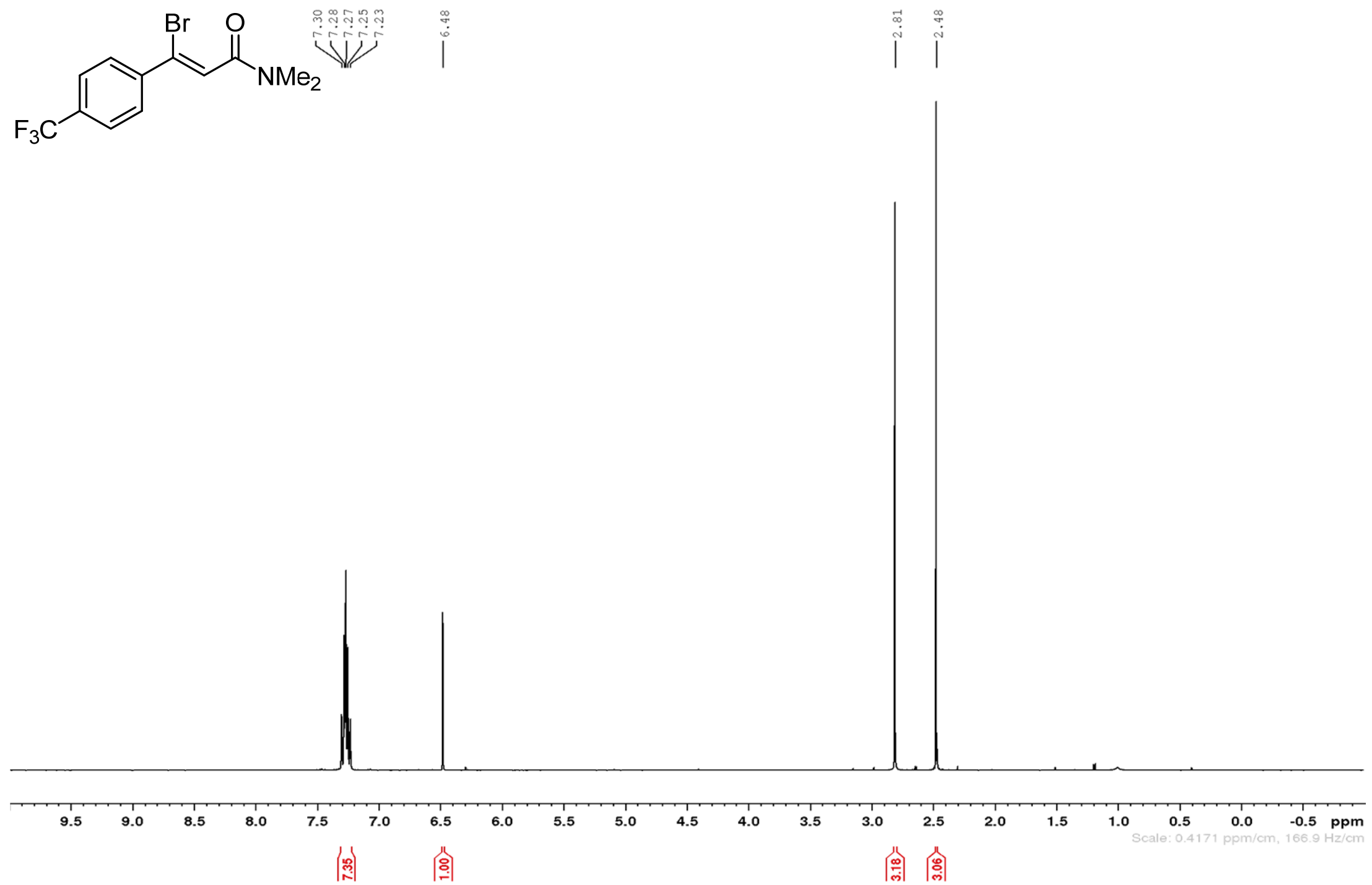
Scheme S50. ${ }^{13} \mathrm{C}\left\{{ }^{1} \mathrm{H}\right\}$ NMR (100 MHz, $\left.\mathrm{C}_{6} \mathrm{D}_{6}, 298 \mathrm{~K}\right)$ of (Z)-3-bromo-N,N-dimethyl-3-(4-(trifluoromethyl)phenyl)acrylamide $(Z-7 e)$

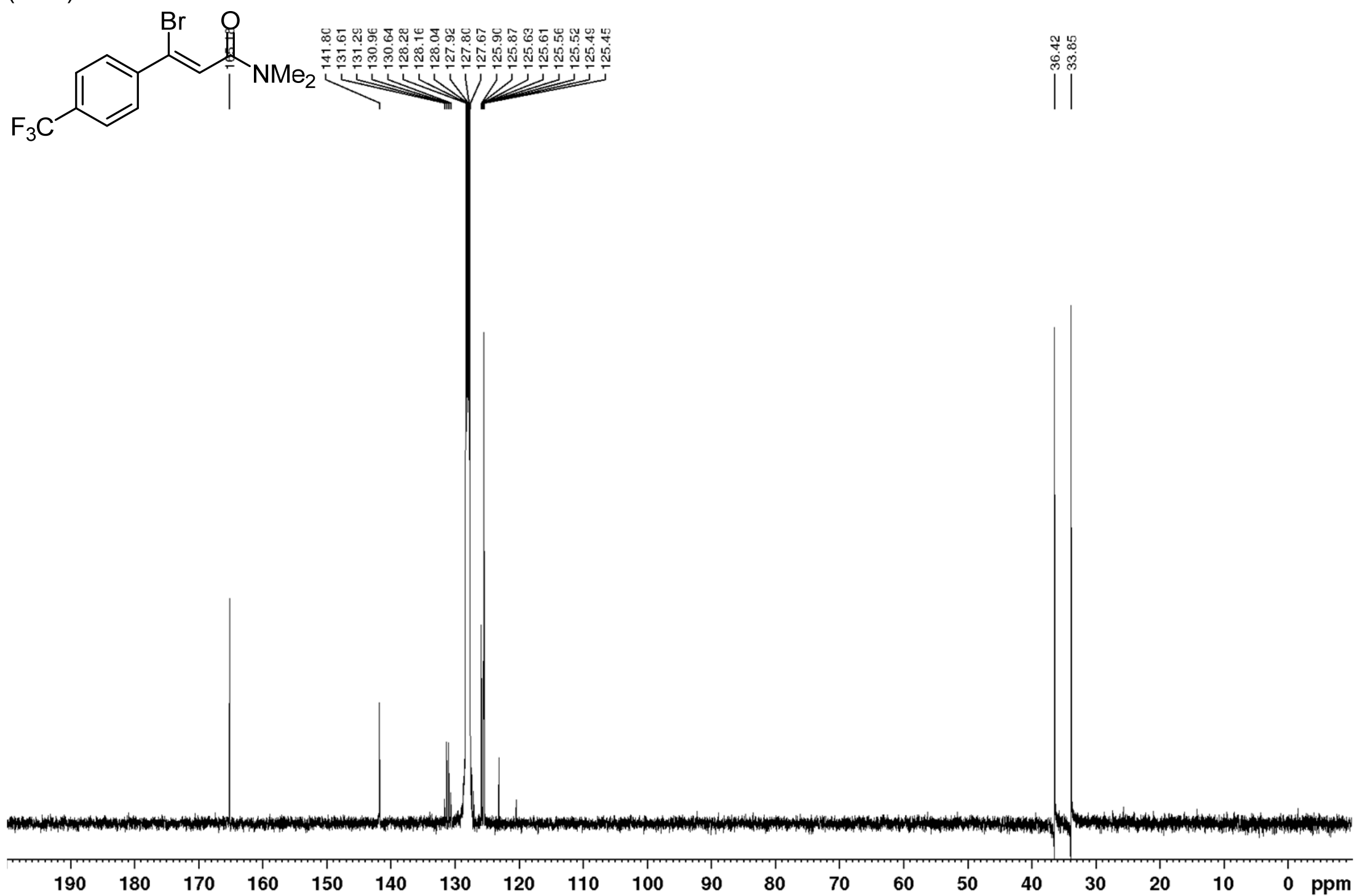


Scheme S51. ${ }^{19} \mathrm{~F}\left\{{ }^{1} \mathrm{H}\right\}$ NMR (659 MHz, $\mathrm{C}_{6} \mathrm{D}_{6}, 298 \mathrm{~K}$ ) of (Z)-3-bromo-N,N-dimethyl-3-(4-(trifluoromethyl)phenyl)acrylamide $(Z-7 e)$<smiles>CNC(=O)C=C(Br)c1ccc(C(F)(F)F)cc1</smiles>

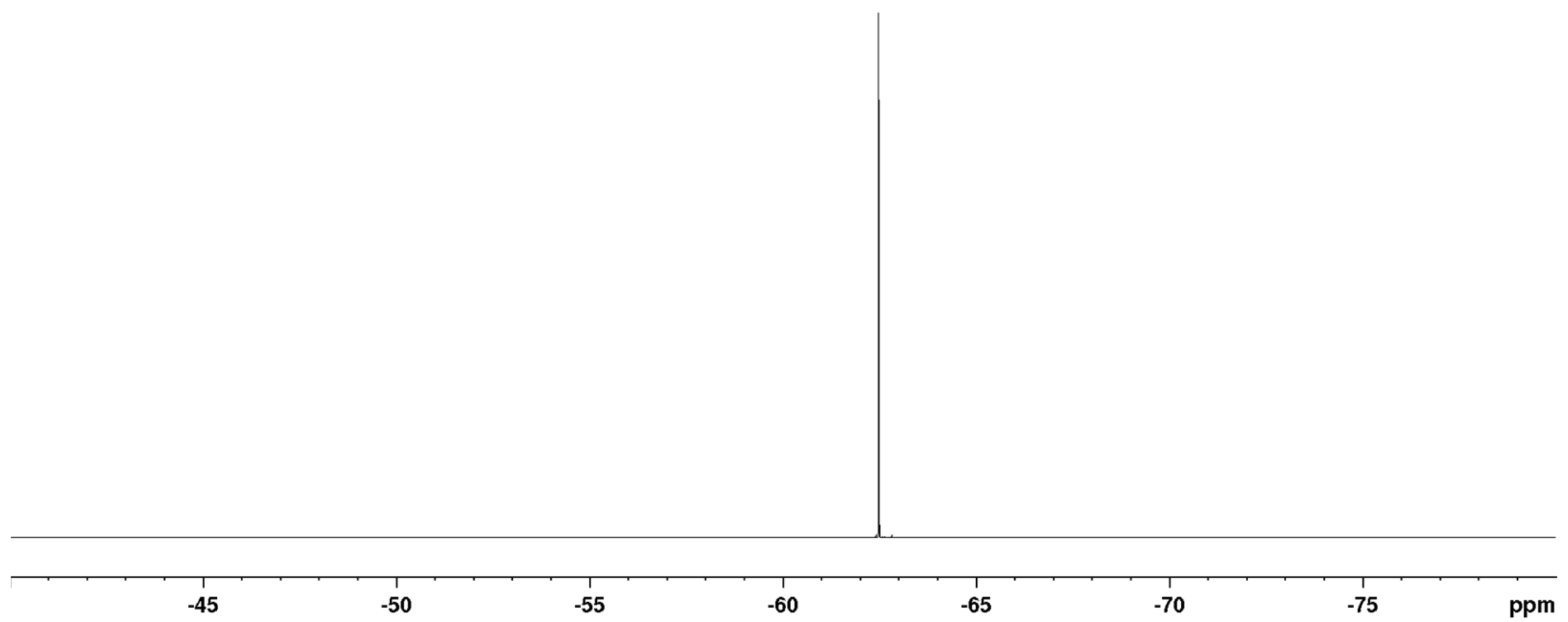


Scheme S52. $\quad{ }^{1} \mathrm{H} \quad \mathrm{NMR} \quad$ spectrum $\quad\left(400 \quad \mathrm{MHz}, \quad \mathrm{C}_{6} \mathrm{D}_{6}, \quad 298 \quad \mathrm{~K}\right) \quad$ of $\quad(E)-3-$ bromo- $N, N$-dimethyl-3-(4(trifluoromethyl)phenyl)acrylamide $(E-7 e)\left(\&=N, N\right.$-dimethyl-3-(4-(trifluoromethyl)phenyl)propiolamide $\left.(6 \mathbf{e}), \#=\mathrm{H}_{2} \mathrm{O}\right)$
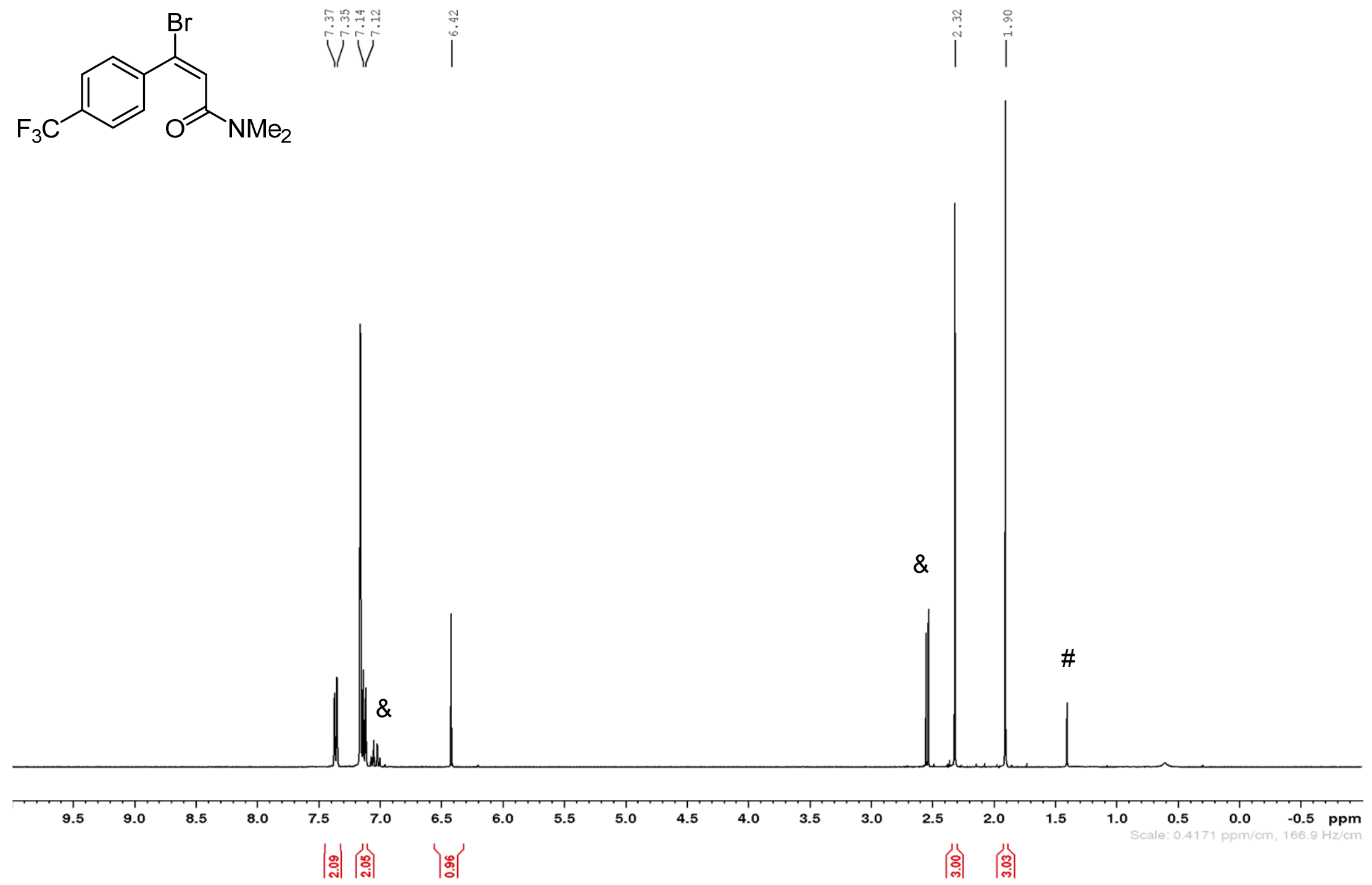
Scheme S53. ${ }^{13} \mathrm{C}\left\{{ }^{1} \mathrm{H}\right\}$ NMR (100 MHz, $\left.\mathrm{C}_{6} \mathrm{D}_{6}, 298 \mathrm{~K}\right)$ of (Z)-3-bromo-N,N-dimethyl-3-(4-(trifluoromethyl)phenyl)acrylamide $(E-7 e)$<smiles>CN(C)C(=O)/C=C(/Br)c1ccc(C(F)(F)F)cc1</smiles>

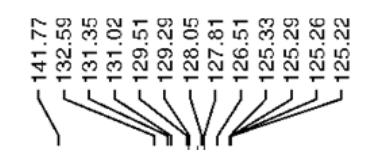


Scheme S54. ${ }^{19} \mathrm{~F}\left\{{ }^{1} \mathrm{H}\right\}$ NMR (659 MHz, $\mathrm{C}_{6} \mathrm{D}_{6}, 298 \mathrm{~K}$ ) of (E)-3-bromo-N,N-dimethyl-3-(4-(trifluoromethyl)phenyl)acrylamide $(E-7 e)$<smiles>CN(C)C(=O)/C=C(/Br)c1ccc(C(F)(F)F)cc1</smiles>

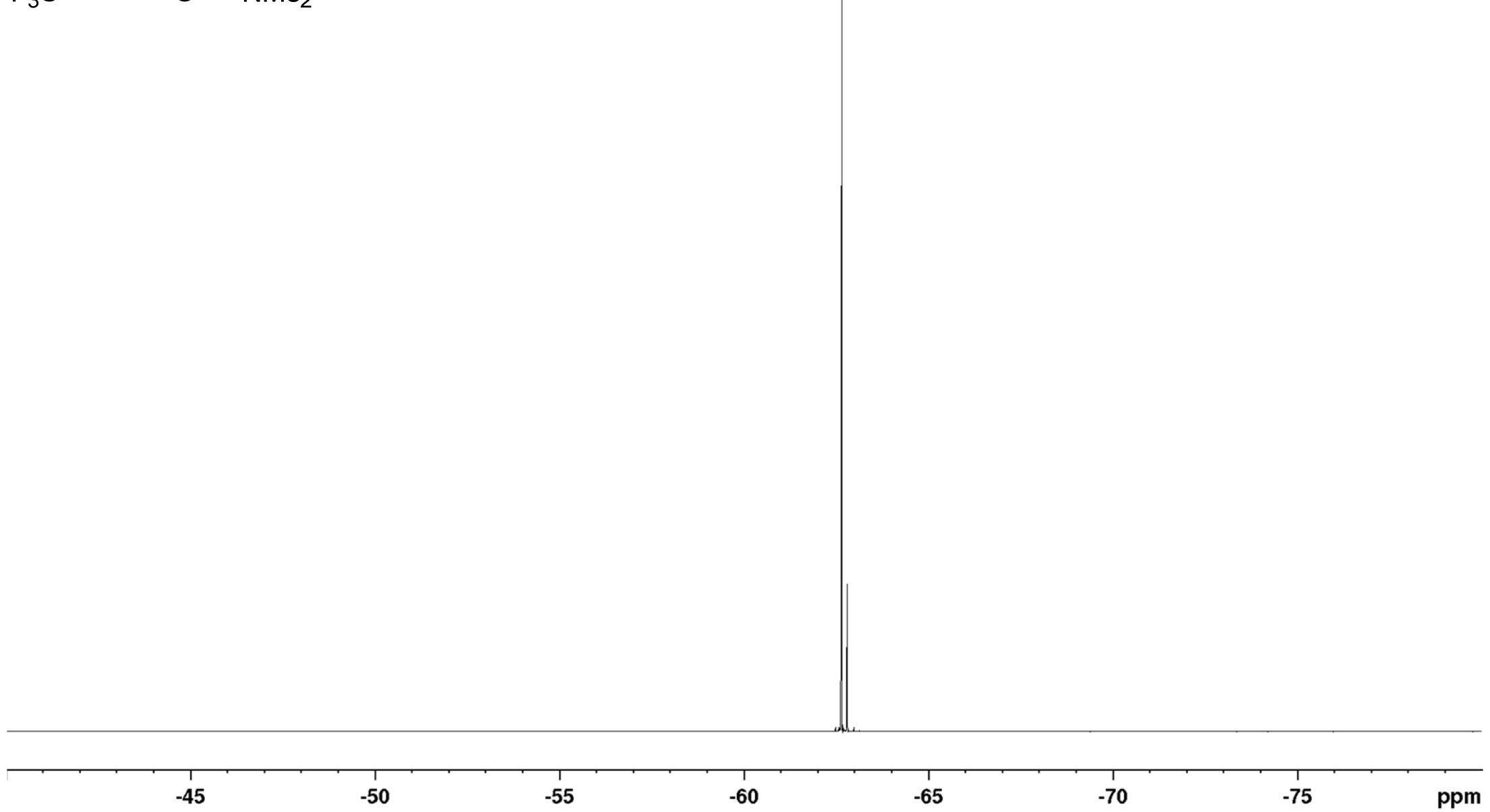


Scheme S55. ${ }^{1} \mathrm{H}$ NMR spectrum (400 MHz, $\mathrm{C}_{6} \mathrm{D}_{6}, 298 \mathrm{~K}$ ) of (Z)-3-bromo-N,N-dimethyl-3-(p-tolyl)acrylamide (Z-7f)

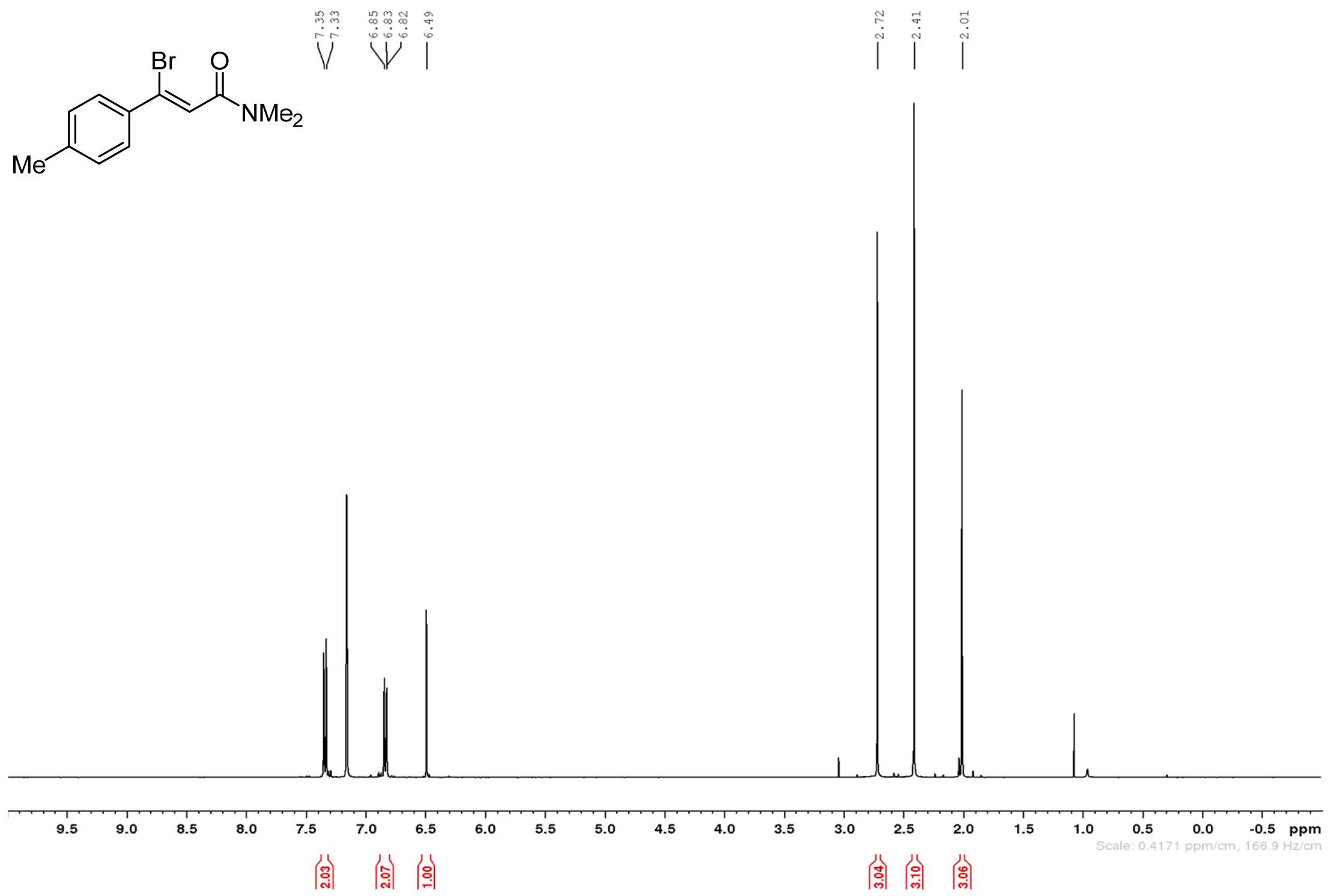


Scheme S56. ${ }^{13} \mathrm{C}\left\{{ }^{1} \mathrm{H}\right\}$ NMR (100 MHz, $\left.\mathrm{C}_{6} \mathrm{D}_{6}, 298 \mathrm{~K}\right)$ of (Z)-3-bromo-N,N-dimethyl-3-(p-tolyl)acrylamide (Z-7f)

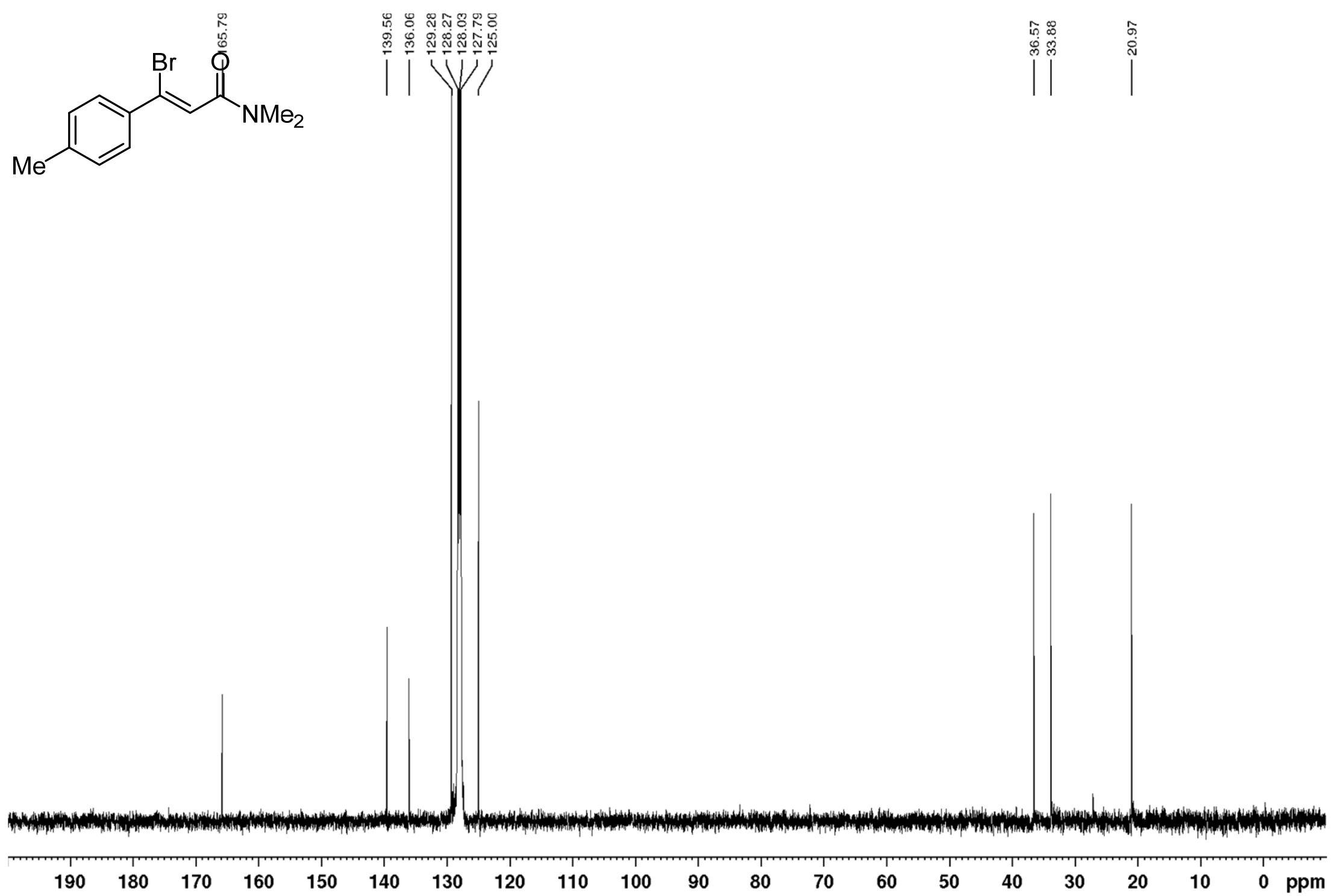


Scheme S57. ${ }^{1} \mathrm{H}$ NMR spectrum $\left(400 \mathrm{MHz}, \mathrm{C}_{6} \mathrm{D}_{6}, 298 \mathrm{~K}\right)$ of $(E)-3$-bromo- $N, N$-dimethyl-3-(p-tolyl)acrylamide $(E-7 f)(\&=$ tert-butyl methyl ether)

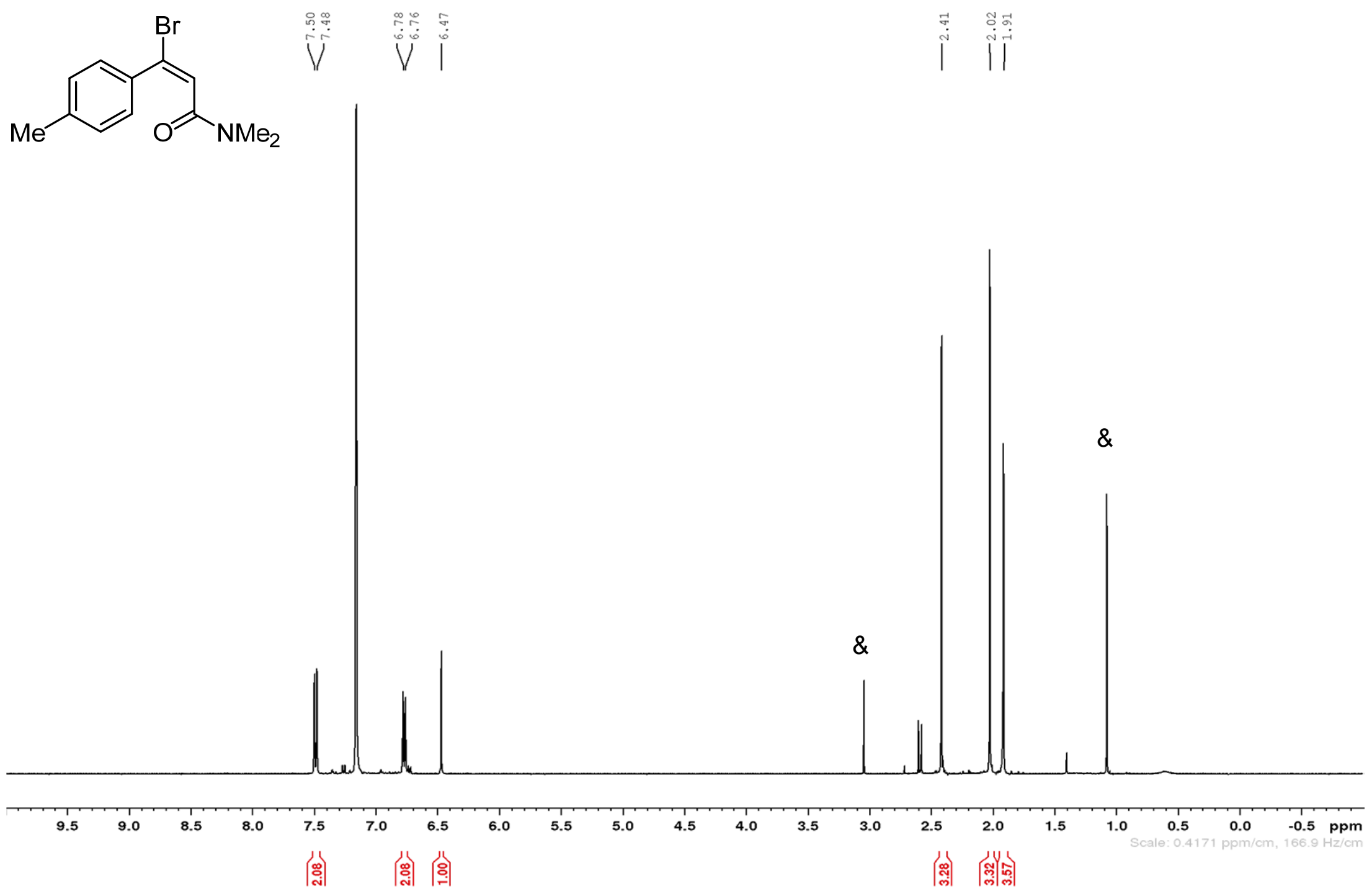


Scheme S58. ${ }^{13} \mathrm{C}\left\{{ }^{1} \mathrm{H}\right\}$ NMR (100 MHz, $\left.\mathrm{C}_{6} \mathrm{D}_{6}, 298 \mathrm{~K}\right)$ of $(E)-3$-bromo- $N, N$-dimethyl-3-( $p$-tolyl)acrylamide $(\boldsymbol{E}$-7f $)$
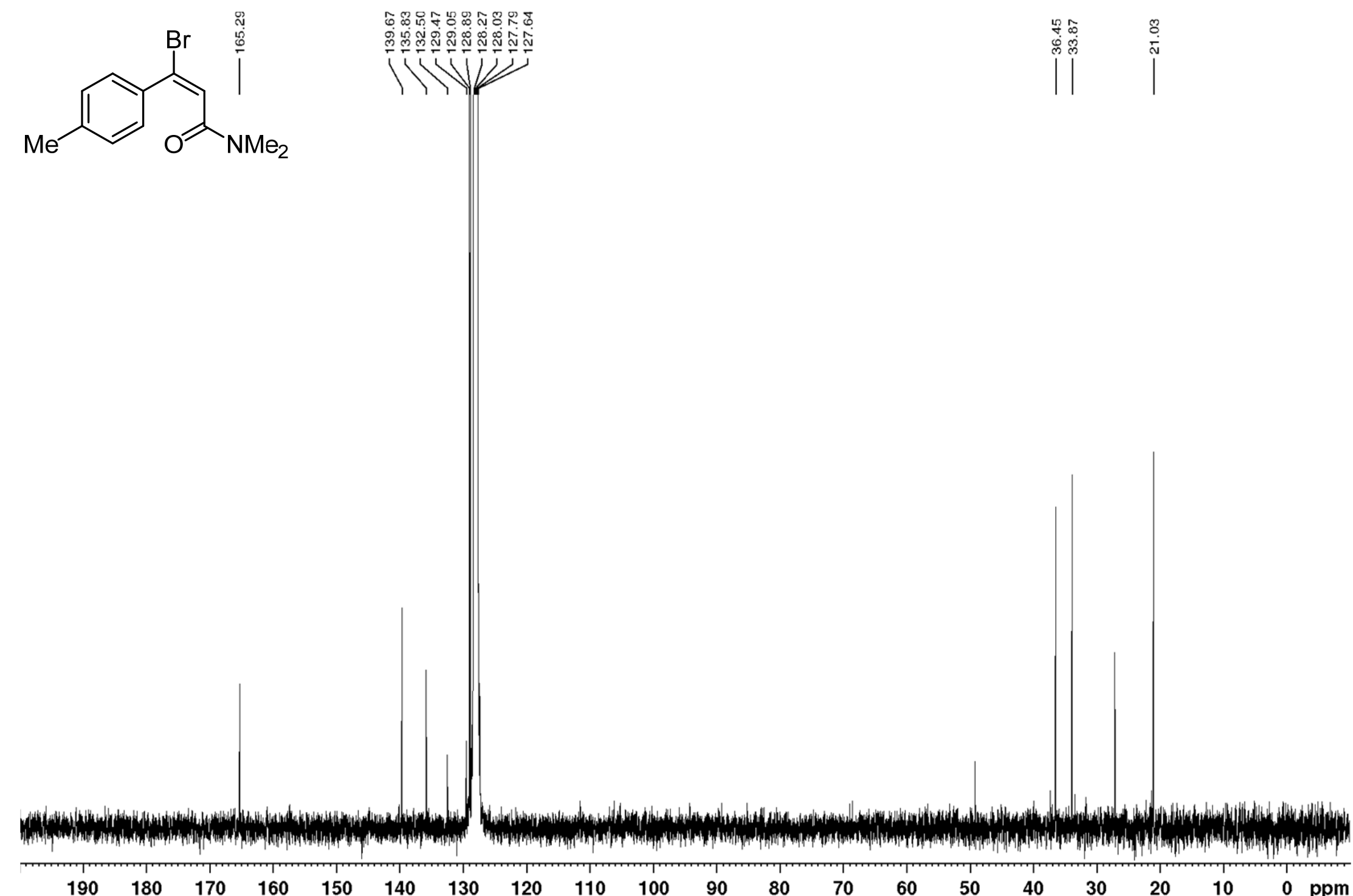
Scheme S59. ${ }^{1} \mathrm{H}$ NMR spectrum (400 MHz, $\left.\mathrm{C}_{6} \mathrm{D}_{6}, 298 \mathrm{~K}\right)$ of (Z)-3-bromo-3-(6-methoxynaphthalen-2-yl)- N,Ndimethylacrylamide $(\mathbf{Z}-\mathbf{7 g})$

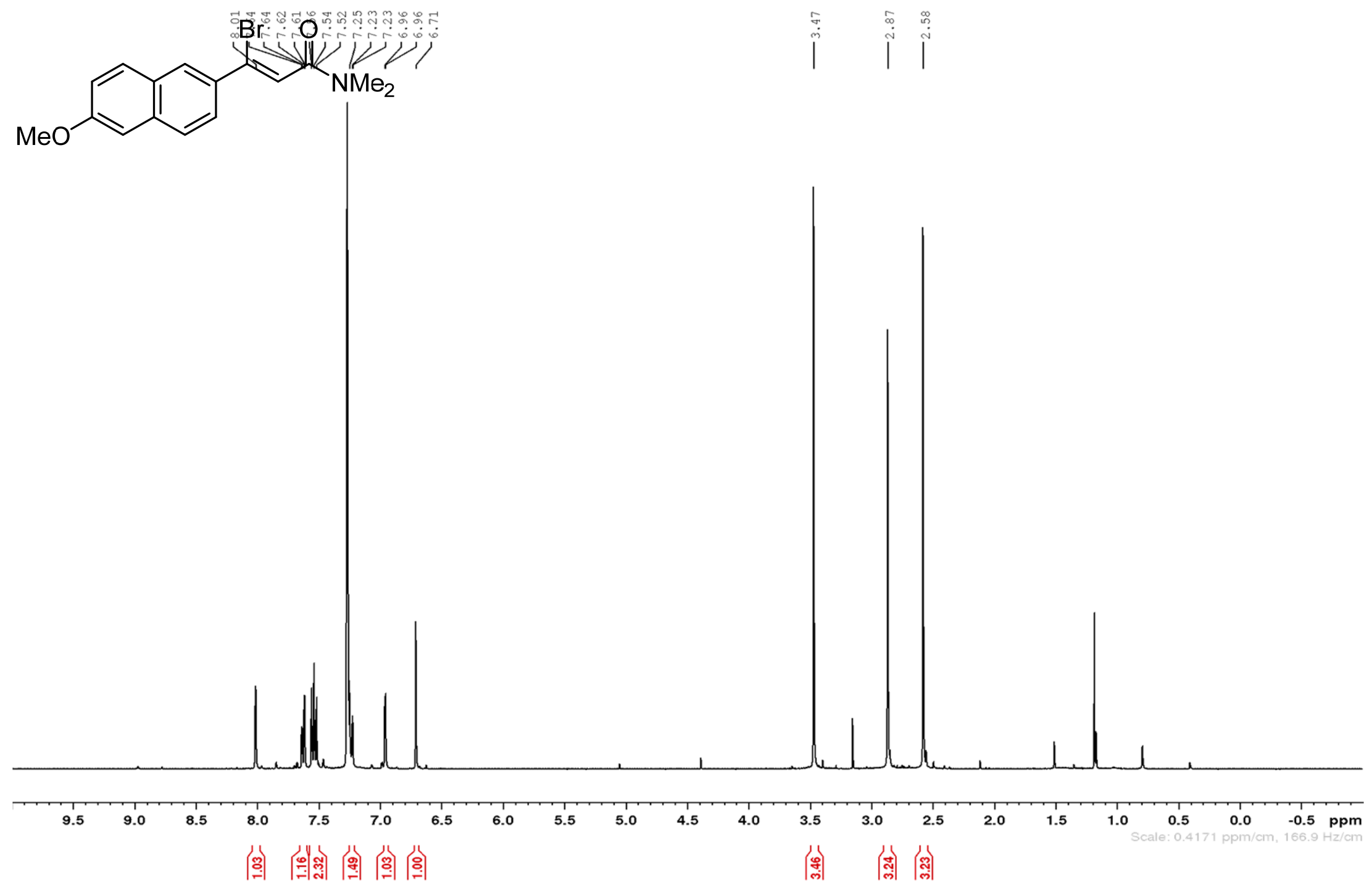


Scheme s60. ${ }^{13} \mathrm{C}\left\{{ }^{1} \mathrm{H}\right\} \quad \mathrm{NMR} \quad\left(100 \quad \mathrm{MHz}, \quad \mathrm{C}_{6} \mathrm{D}_{6}, \quad 298 \quad \mathrm{~K}\right) \quad$ of $\quad(Z)-3-$ bromo-3-(6-methoxynaphthalen-2-yl)- $N, N-$ dimethylacrylamide $(\mathbf{Z}-\mathbf{7 g})$

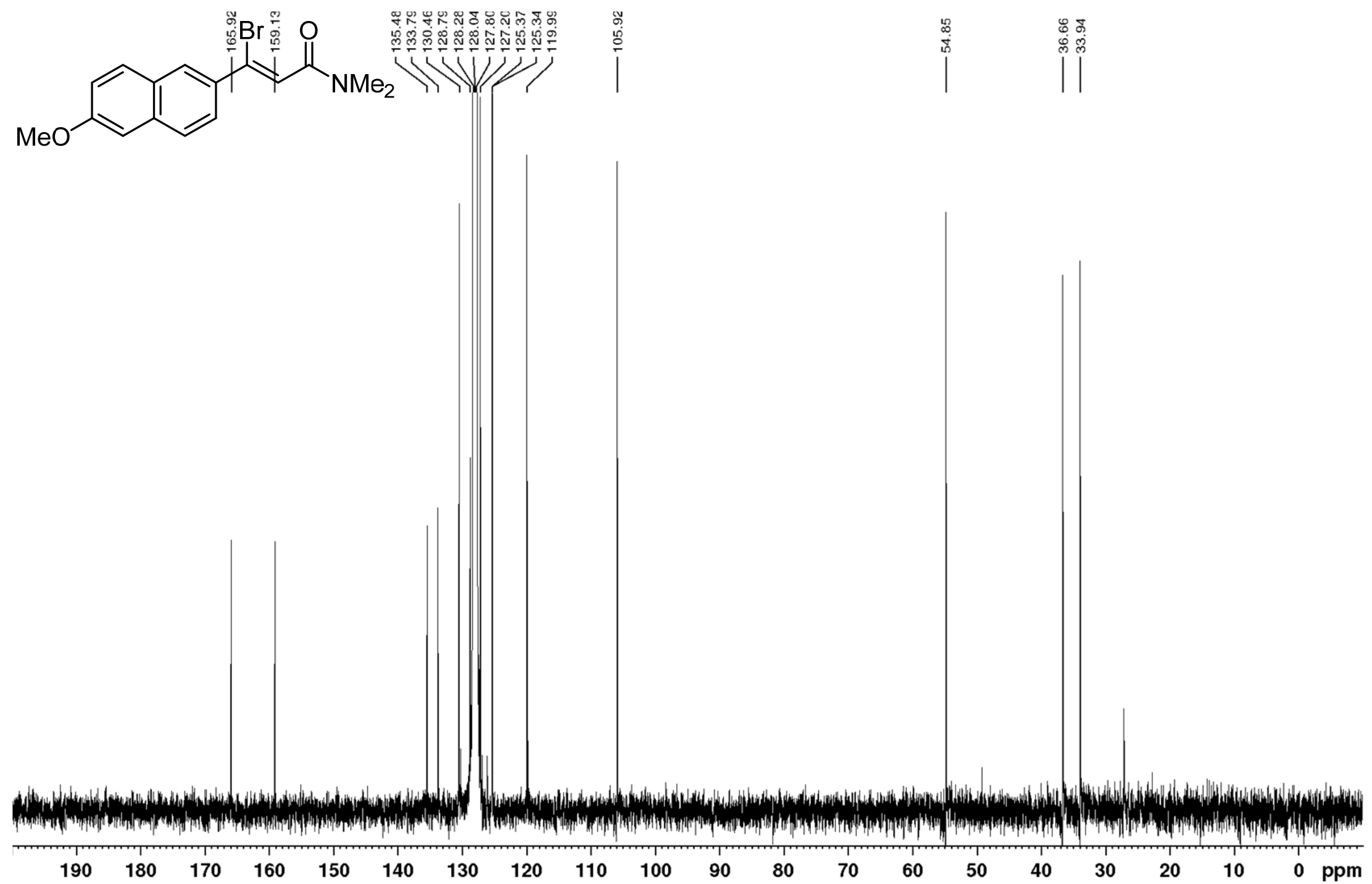


Scheme S61. ${ }^{1} \mathrm{H}$ NMR spectrum (400 MHz, $\left.\mathrm{C}_{6} \mathrm{D}_{6}, 298 \mathrm{~K}\right)$ of (E)-3-bromo-3-(6-methoxynaphthalen-2-yl)- $N, N-$ dimethylacrylamide $(\boldsymbol{E}-\mathbf{7 g})(\&=3-(6-m e t h o x y n a p h t h a l e n-2-y l)-N, N$-dimethylpropiolamide $(\mathbf{6 g}))$

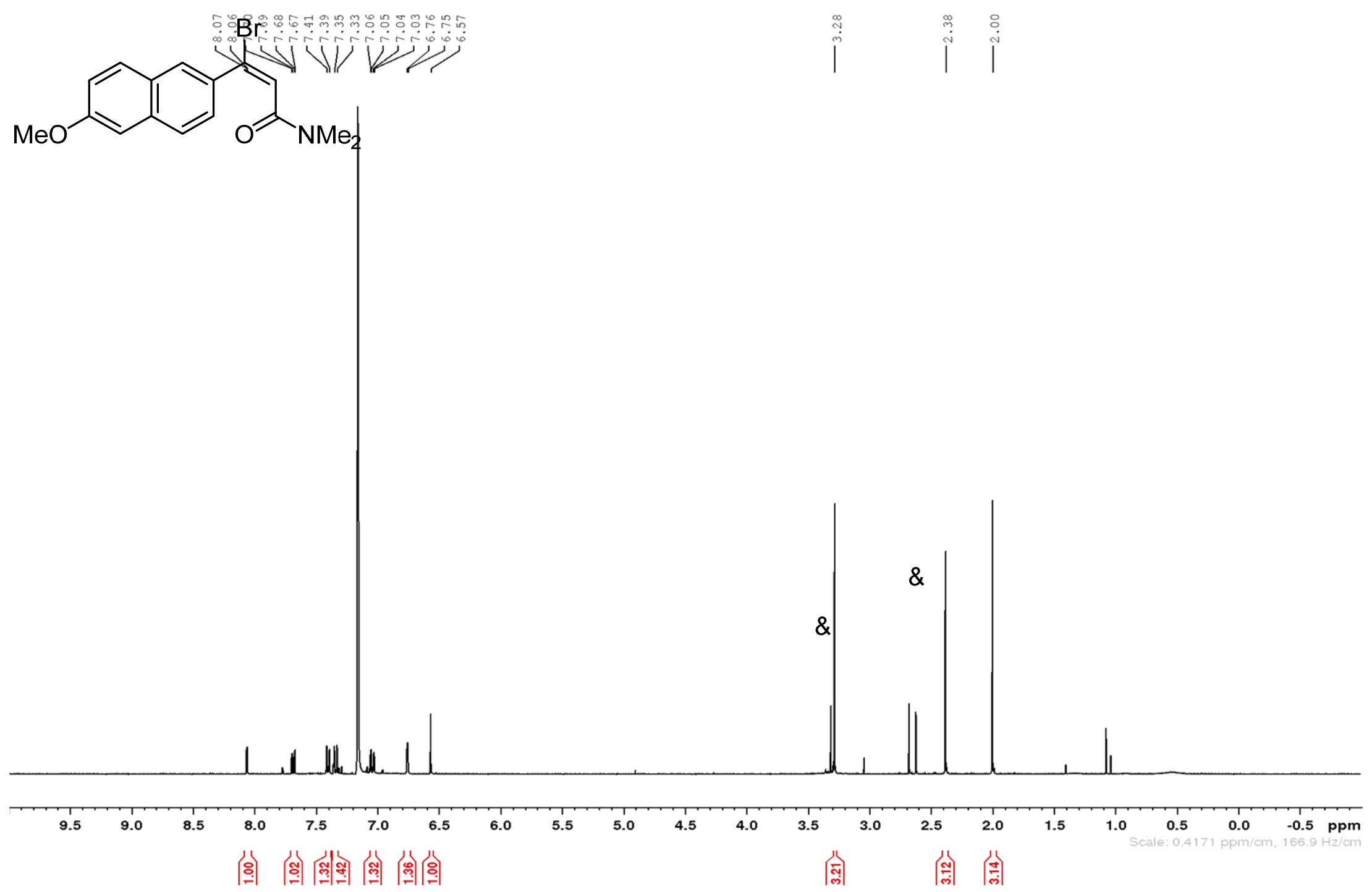


Scheme S62. ${ }^{13} \mathrm{C}\left\{{ }^{1} \mathrm{H}\right\} \quad \mathrm{NMR} \quad\left(100 \quad \mathrm{MHz}, \quad \mathrm{C}_{6} \mathrm{D}_{6}, \quad 298 \quad \mathrm{~K}\right) \quad$ of $\quad(E)-3-$ bromo-3-(6-methoxynaphthalen-2-yl)- $N, N-$ dimethylacrylamide $(\boldsymbol{E}-\mathbf{7 g})$
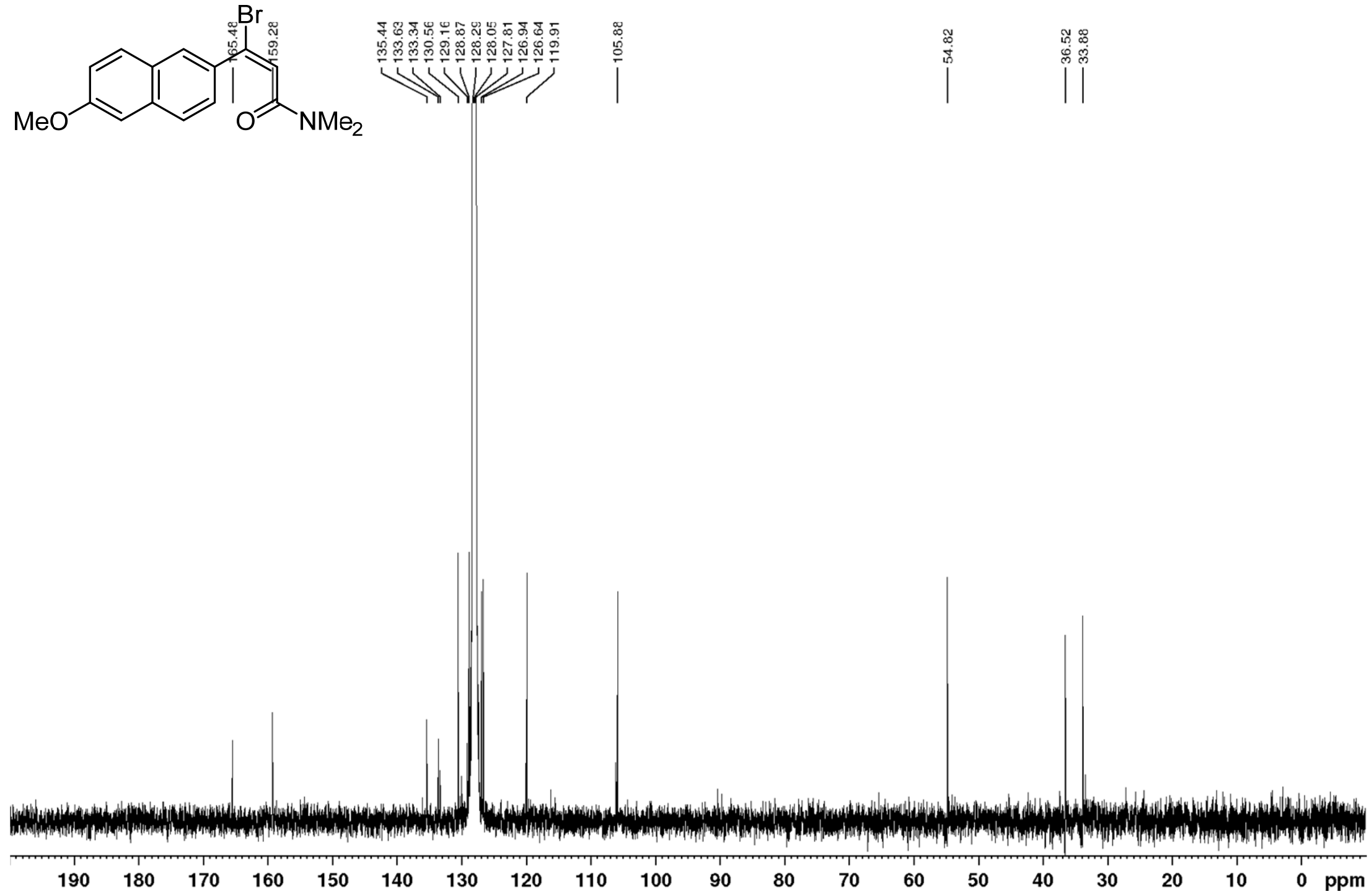
Scheme S63. ${ }^{1} \mathrm{H}$ NMR spectrum $\left(400 \mathrm{MHz}, \mathrm{C}_{6} \mathrm{D}_{6}, 298 \mathrm{~K}\right)$ of (Z)-3-bromo-3-cyclopropyl- $N, N$-dimethylacrylamide $(7 \mathbf{h})(\&=$ 3-cyclopropyl- $N, N$-dimethylpropiolamide (6h))
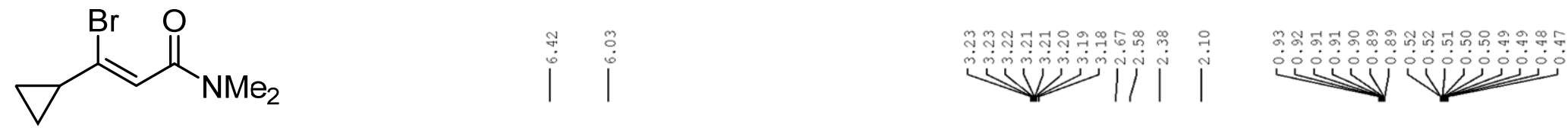

$\&$

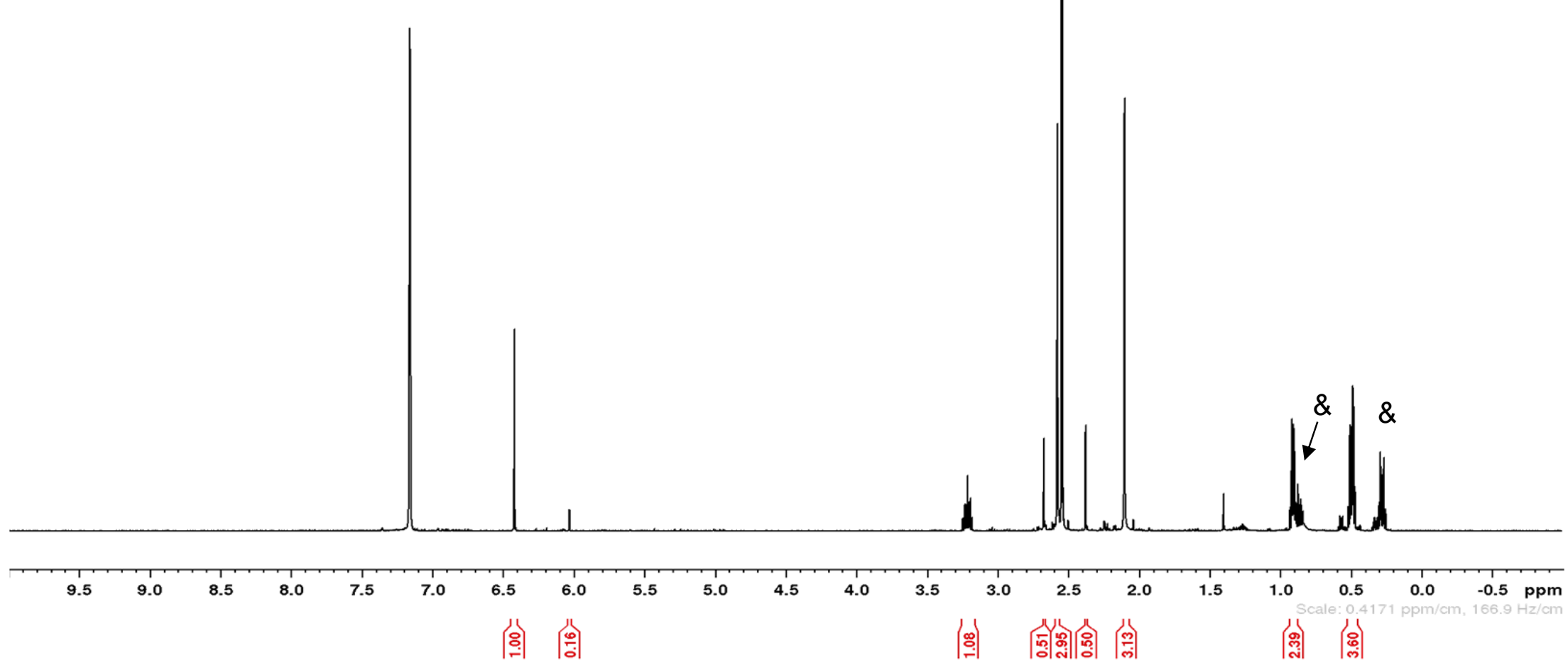


Scheme S64. ${ }^{13} \mathrm{C}\left\{{ }^{1} \mathrm{H}\right\}$ NMR (100 MHz, $\left.\mathrm{C}_{6} \mathrm{D}_{6}, 298 \mathrm{~K}\right)$ of (Z)-3-bromo-3-cyclopropyl- $N, N$-dimethylacrylamide (7h)

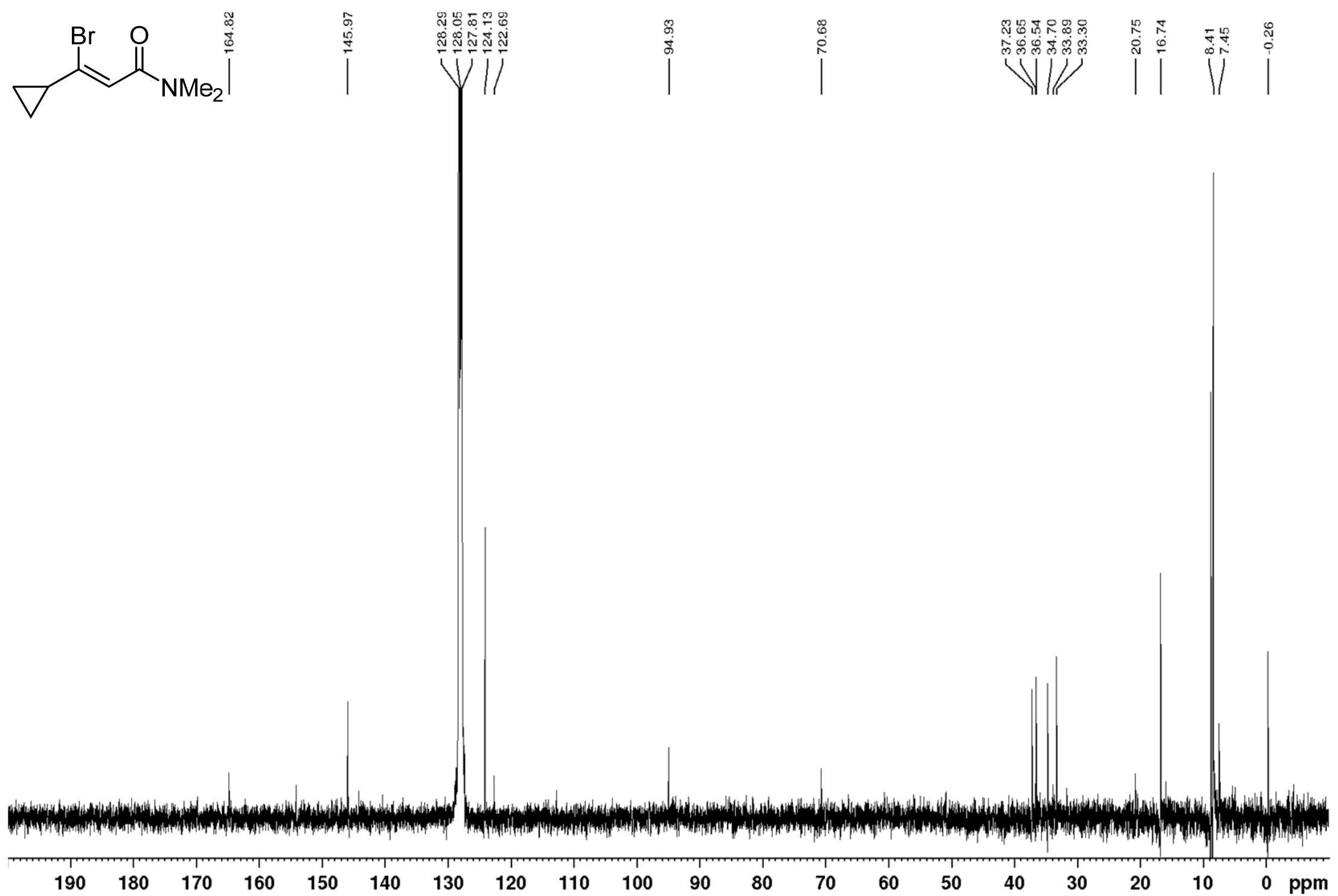


Scheme S65. ${ }^{1} \mathrm{H}$ NMR spectrum $\left(400 \mathrm{MHz}, \mathrm{CD}_{2} \mathrm{Cl}_{2}, 298 \mathrm{~K}\right)$ of (Z)-3-bromo-3-cyclohexyl-N,N-dimethylacrylamide $(7 \mathbf{i})(\&=$ 3-cyclohexyl-N,N-dimethylpropiolamide (6i))<smiles>CNC(=O)C=C(Br)C1CCCCC1</smiles>

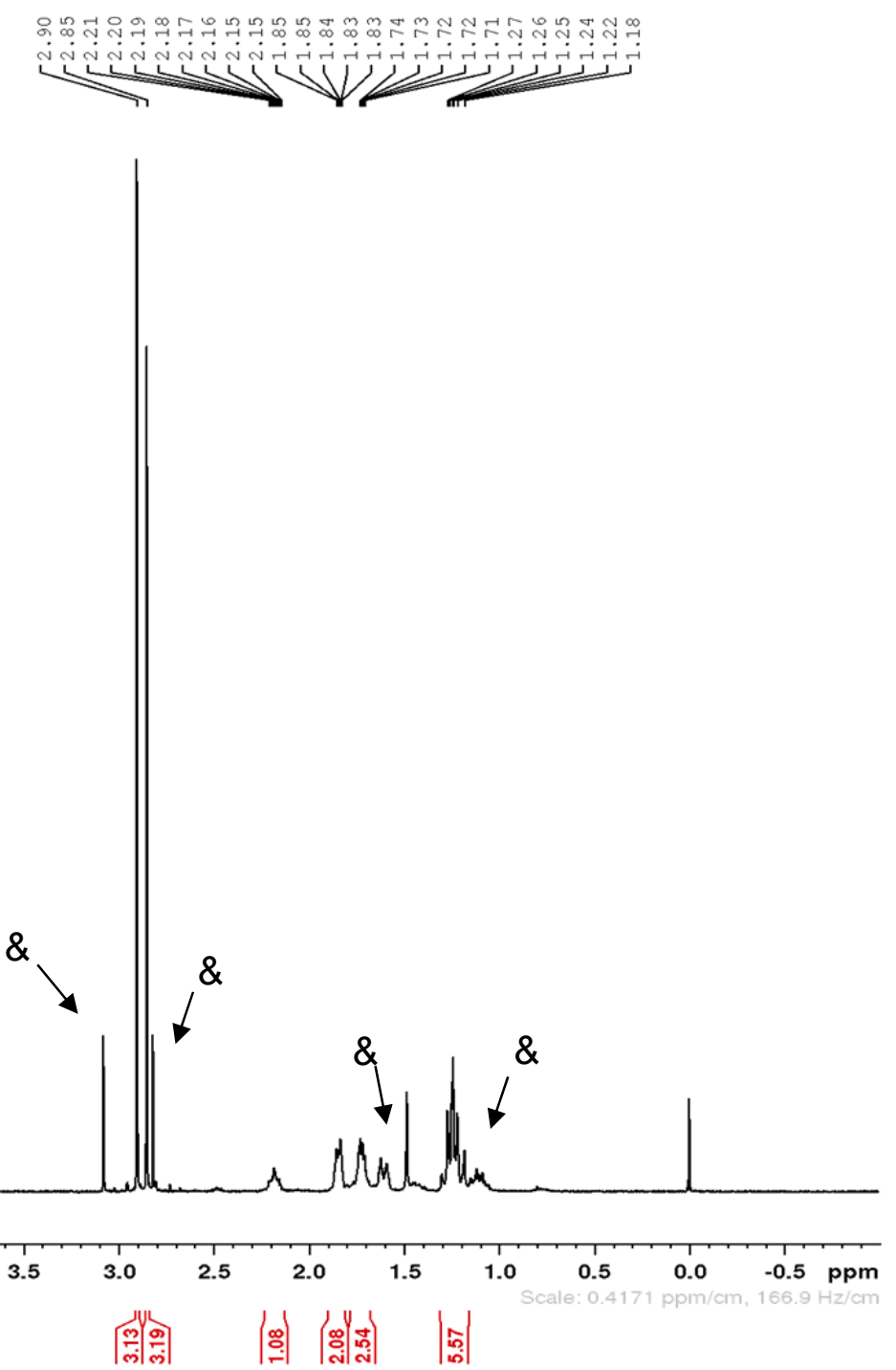


Scheme S66. ${ }^{13} \mathrm{C}\left\{{ }^{1} \mathrm{H}\right\}$ NMR (100 MHz, $\left.\mathrm{CD}_{2} \mathrm{Cl}_{2}, 298 \mathrm{~K}\right)$ of (Z)-3-bromo-3-cyclohexyl-N,N-dimethylacrylamide (7i)
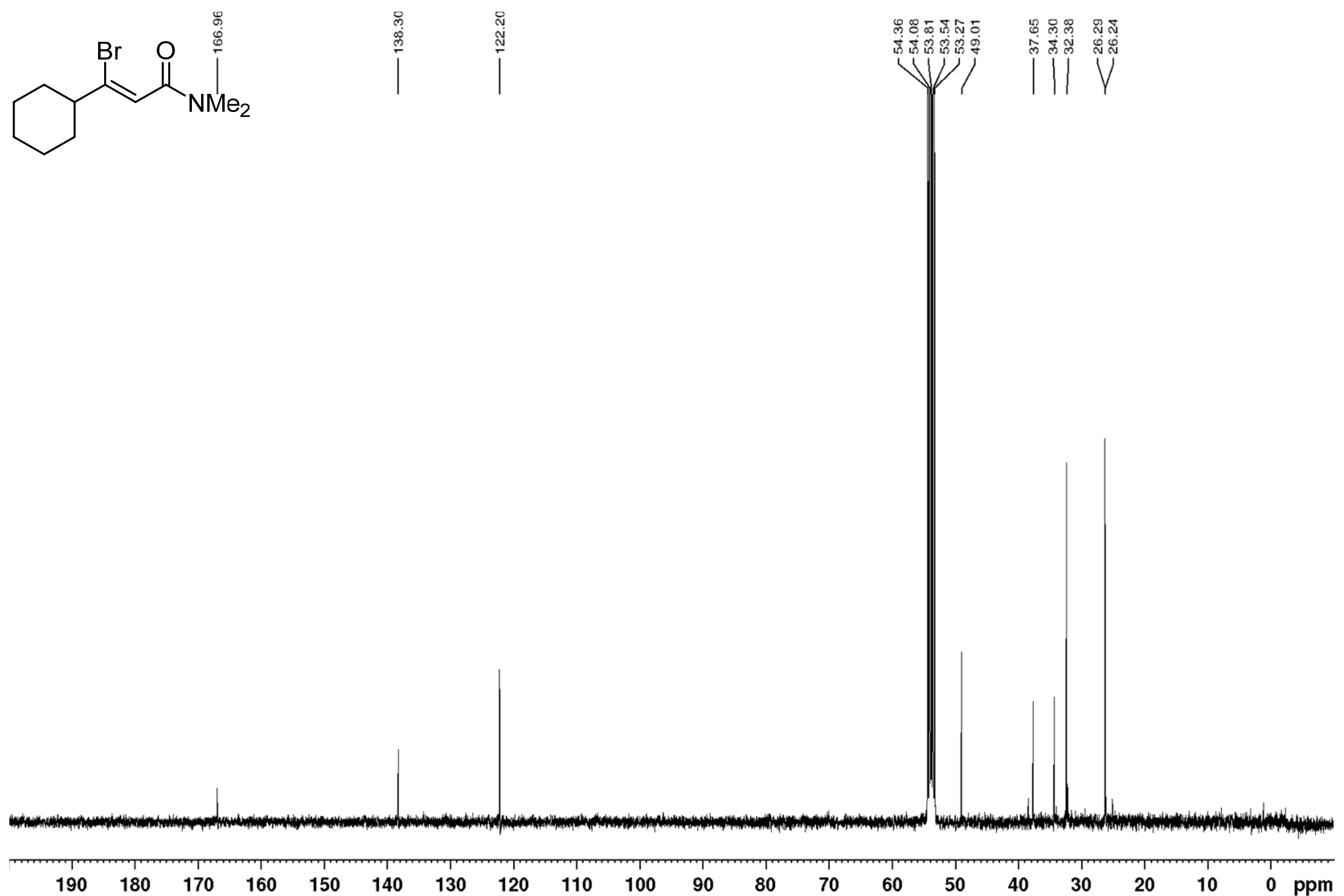
Scheme S67. ${ }^{1} \mathrm{H}$ NMR spectrum $\left(500 \mathrm{MHz}, \mathrm{CD}_{2} \mathrm{Cl}_{2}, 298 \mathrm{~K}\right)$ of ethyl 3-bromo-3-cyclohexylacrylate $(Z: E=53: 47)(7 \mathbf{j})(\&=$ ethyl 3-cyclohexylpropiolate $(6 \mathbf{j}))$<smiles>CCOC(=O)C=C(Br)C1CCCCC1</smiles>

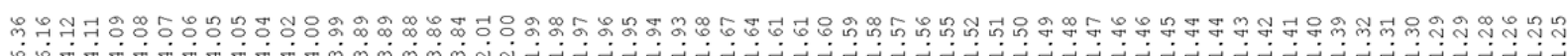

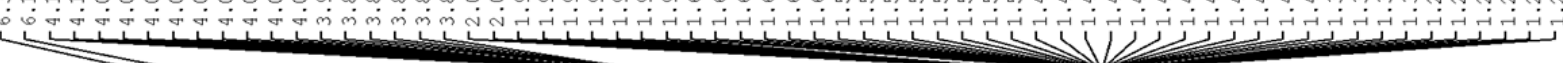
\&

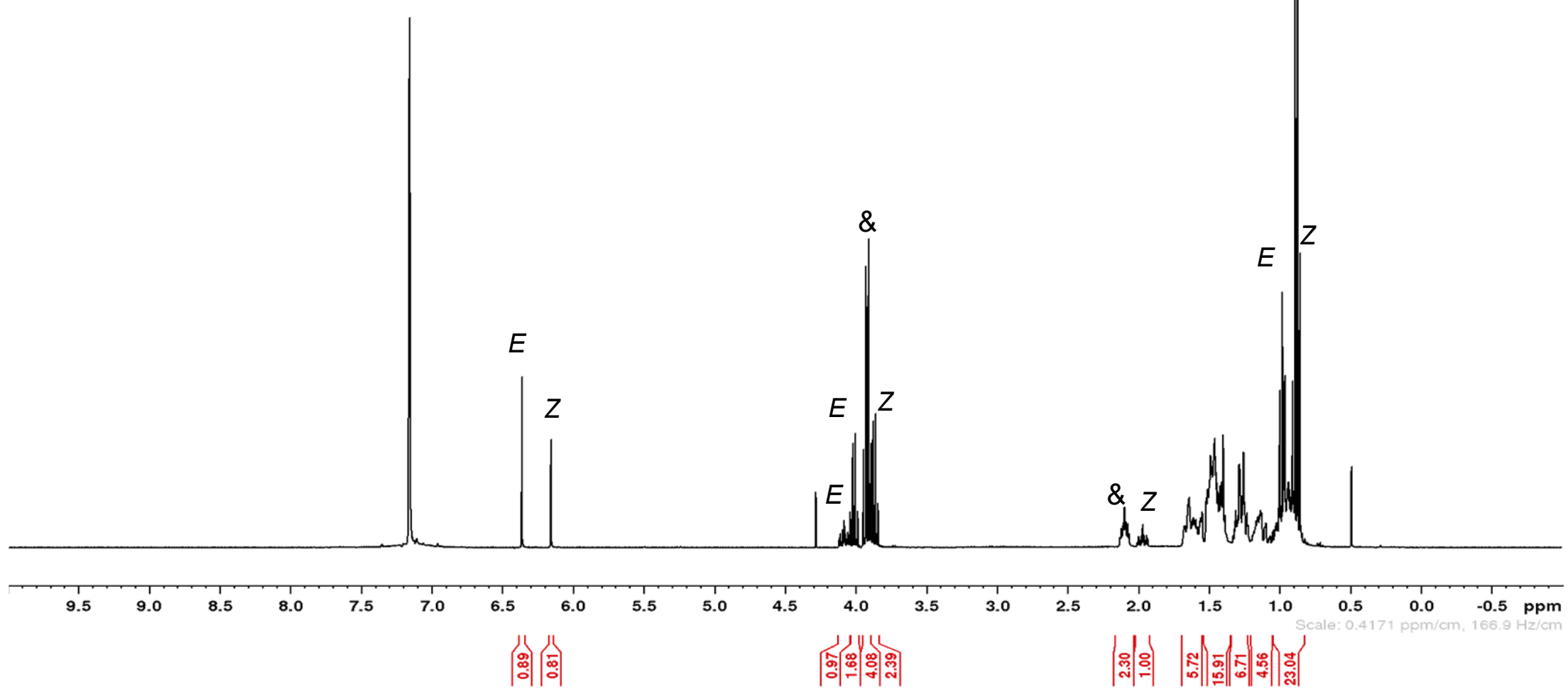


Scheme S68. ${ }^{13} \mathrm{C}\left\{{ }^{1} \mathrm{H}\right\} \mathrm{NMR}\left(125 \mathrm{MHz}, \mathrm{CD}_{2} \mathrm{Cl}_{2}, 298 \mathrm{~K}\right)$ of ethyl 3-bromo-3-cyclohexylacrylate $(Z: E=53: 47)(7 \mathrm{j})($ containing ethyl 3-cyclohexylpropiolate (6j))

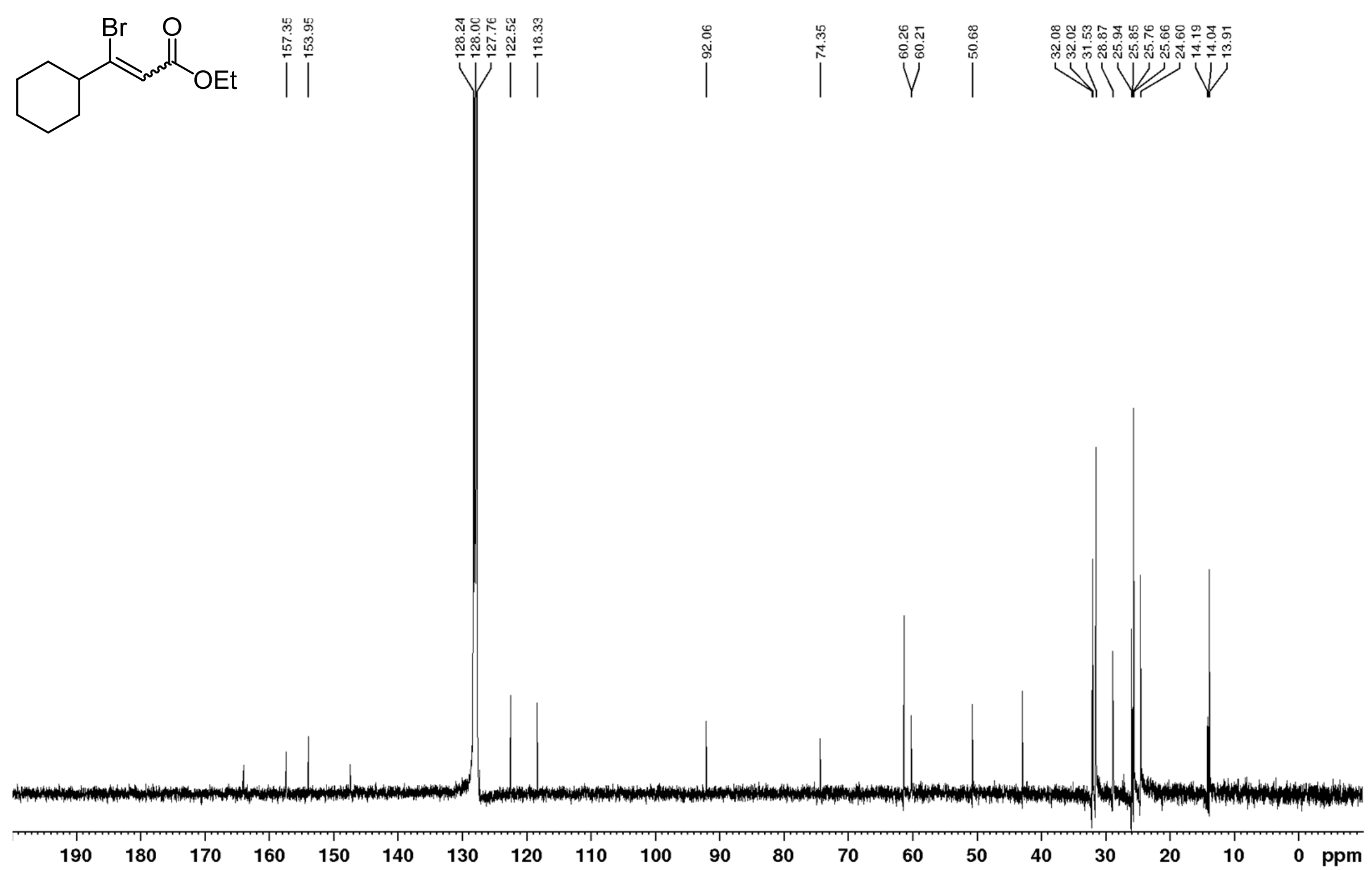


Scheme S69. ${ }^{1} \mathrm{H}$ NMR spectrum (400 MHz, $\left.\mathrm{C}_{6} \mathrm{D}_{6}, 298 \mathrm{~K}\right)$ of $(2 Z, 4 E)-3-$ bromo-N,N,4-trimethyl-5-phenylpenta-2,4dienamide $(\mathbf{7 k})\left(\#=\mathrm{H}_{2} \mathrm{O}\right)$
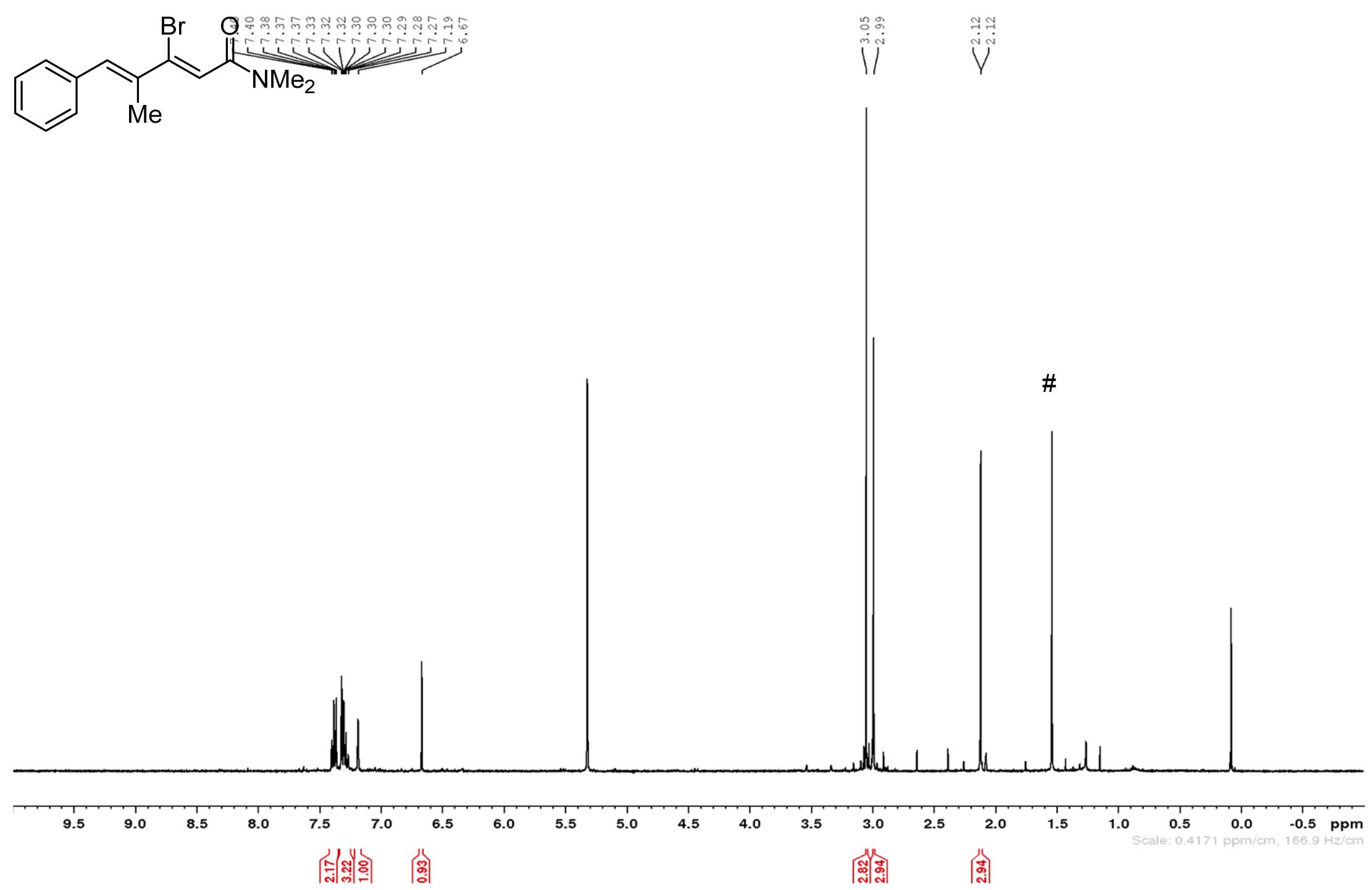
Scheme S70. ${ }^{13} \mathrm{C}\left\{{ }^{1} \mathrm{H}\right\}$ NMR (100 MHz, $\left.\mathrm{C}_{6} \mathrm{D}_{6}, 298 \mathrm{~K}\right)$ of $(2 Z, 4 E)-3-$ bromo-N,N,4-trimethyl-5-phenylpenta-2,4-dienamide (7k)
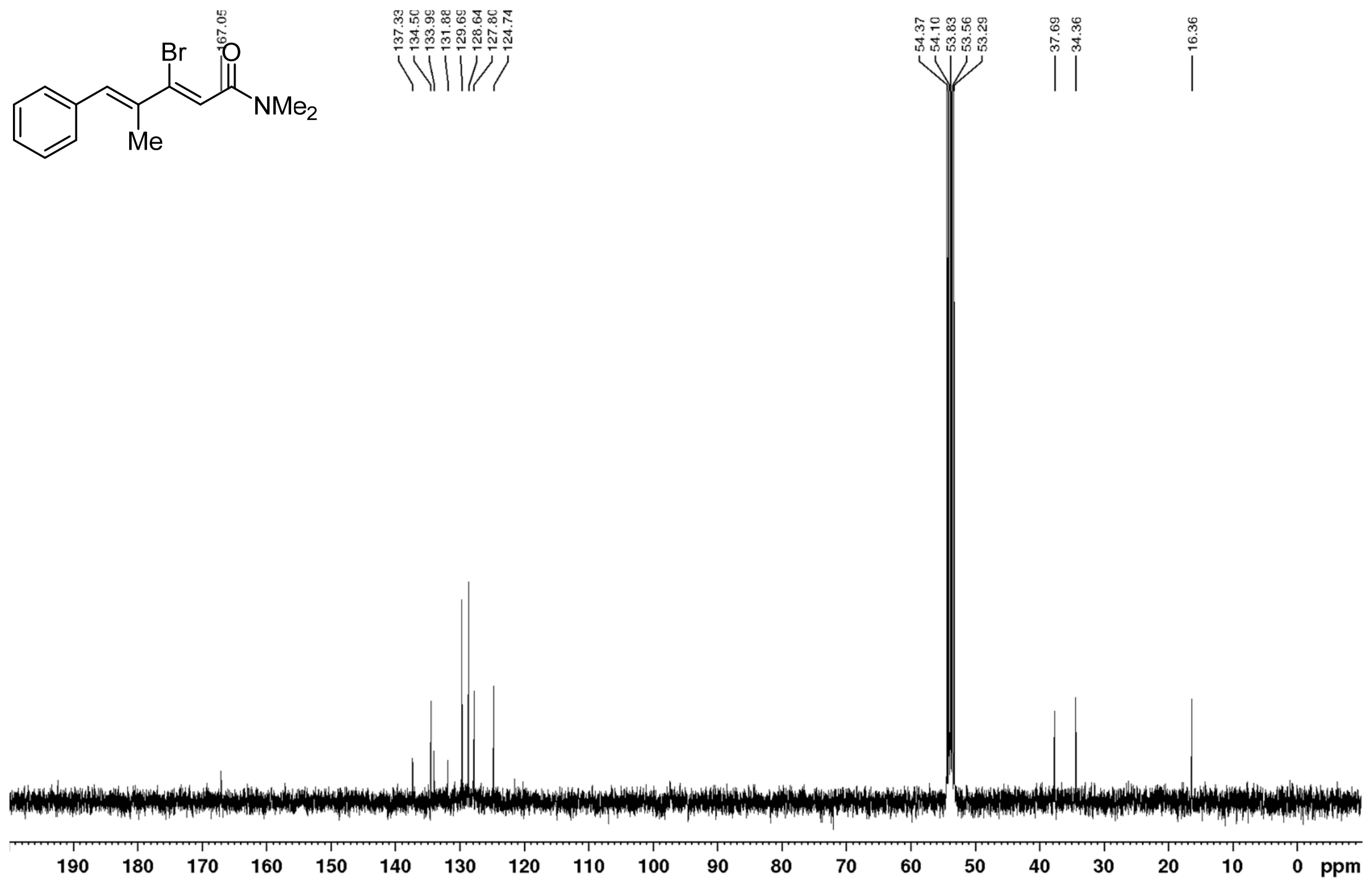
Scheme S71. ${ }^{1} \mathrm{H}$ NMR spectrum (400 MHz, $\left.\mathrm{C}_{6} \mathrm{D}_{6}, 298 \mathrm{~K}\right)$ of afford (Z)-3-bromo-2-methylbut-2-enoic acid $(E: Z=91: 9)(9)$<smiles>CC(Br)=C(C)C(=O)O</smiles>

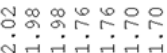

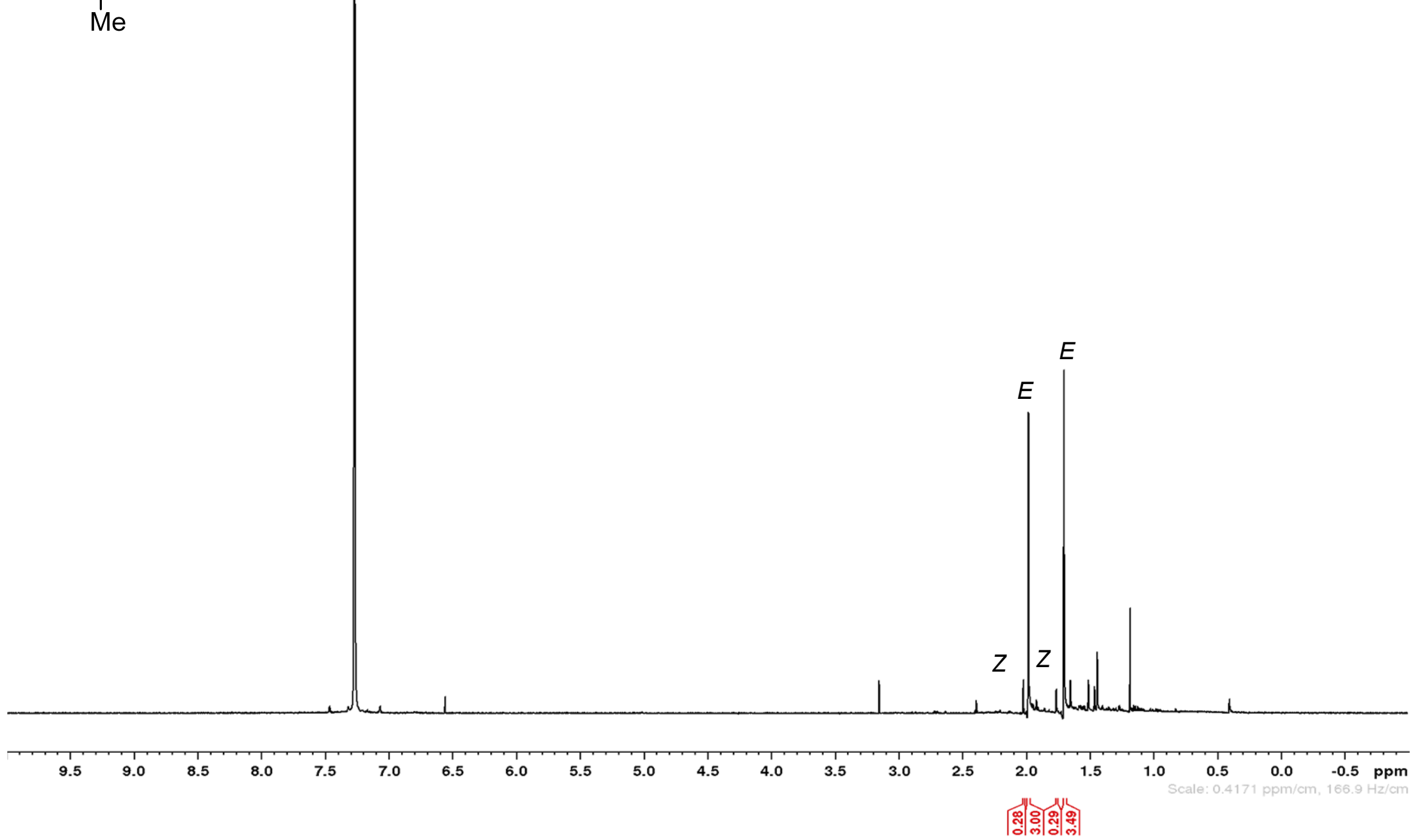


Scheme S72. ${ }^{13} \mathrm{C}\left\{{ }^{1} \mathrm{H}\right\}$ NMR (100 MHz, $\left.\mathrm{C}_{6} \mathrm{D}_{6}, 298 \mathrm{~K}\right)$ of afford (Z)-3-bromo-2-methylbut-2-enoic acid $(E: Z=91: 9)(9)$<smiles>CC(Br)=C(C)C(=[O+])O</smiles>

$$
\left.\right|^{\substack{0 \\ 0}}
$$
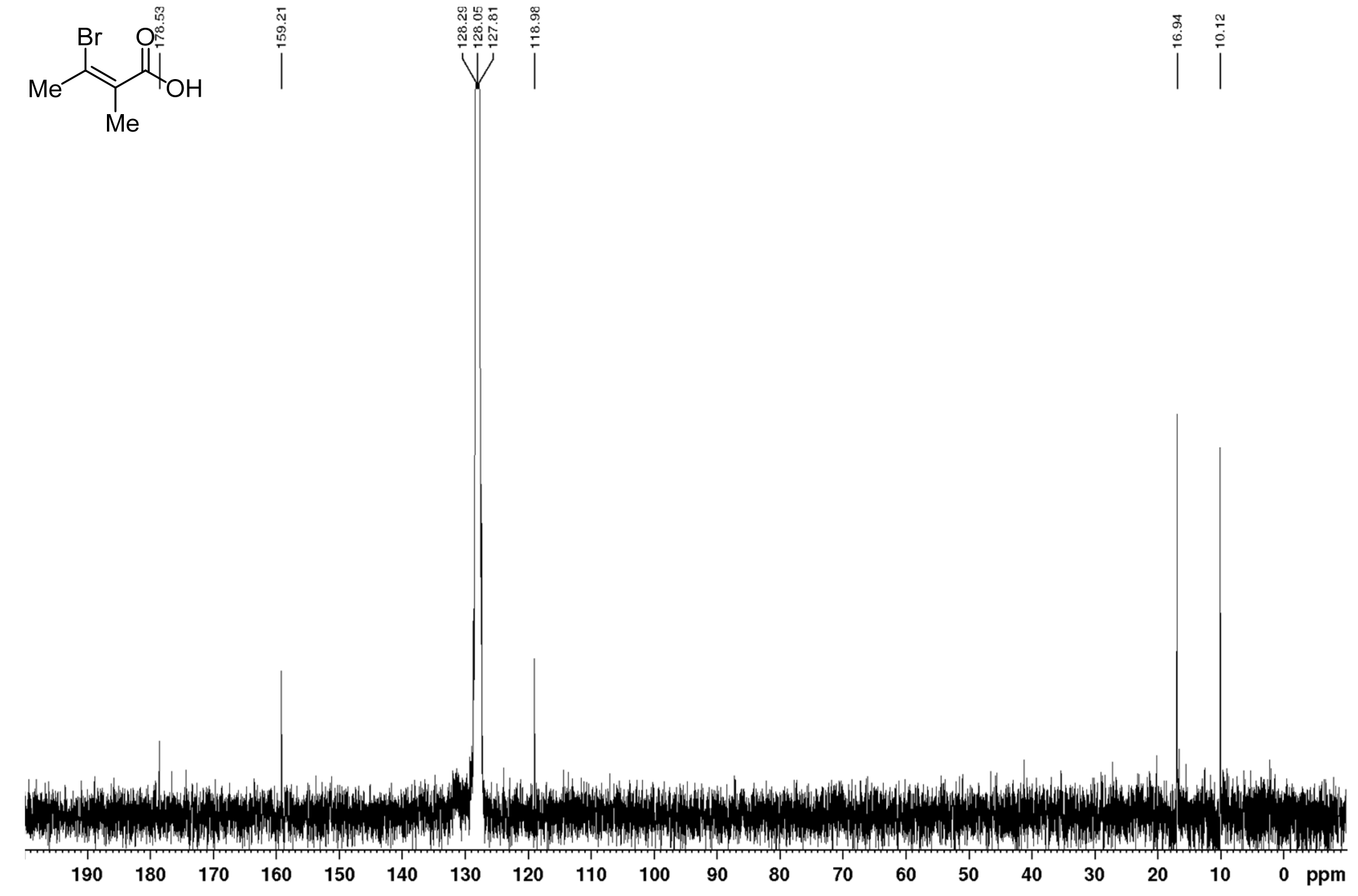
Scheme S73. ${ }^{1} \mathrm{H}$ NMR spectrum $\left(400 \mathrm{MHz}, \mathrm{CD}_{2} \mathrm{Cl}_{2}, 298 \mathrm{~K}\right)$ of 2-(2-bromoethyl)-1,1'-biphenyl (14) (\& = triphenylmethane, \# $=\mathrm{H}_{2} \mathrm{O}, \$=$ cyclohexane)
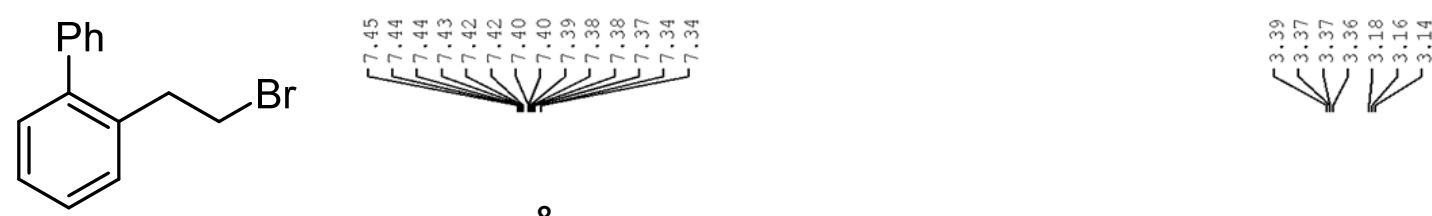

$\&$

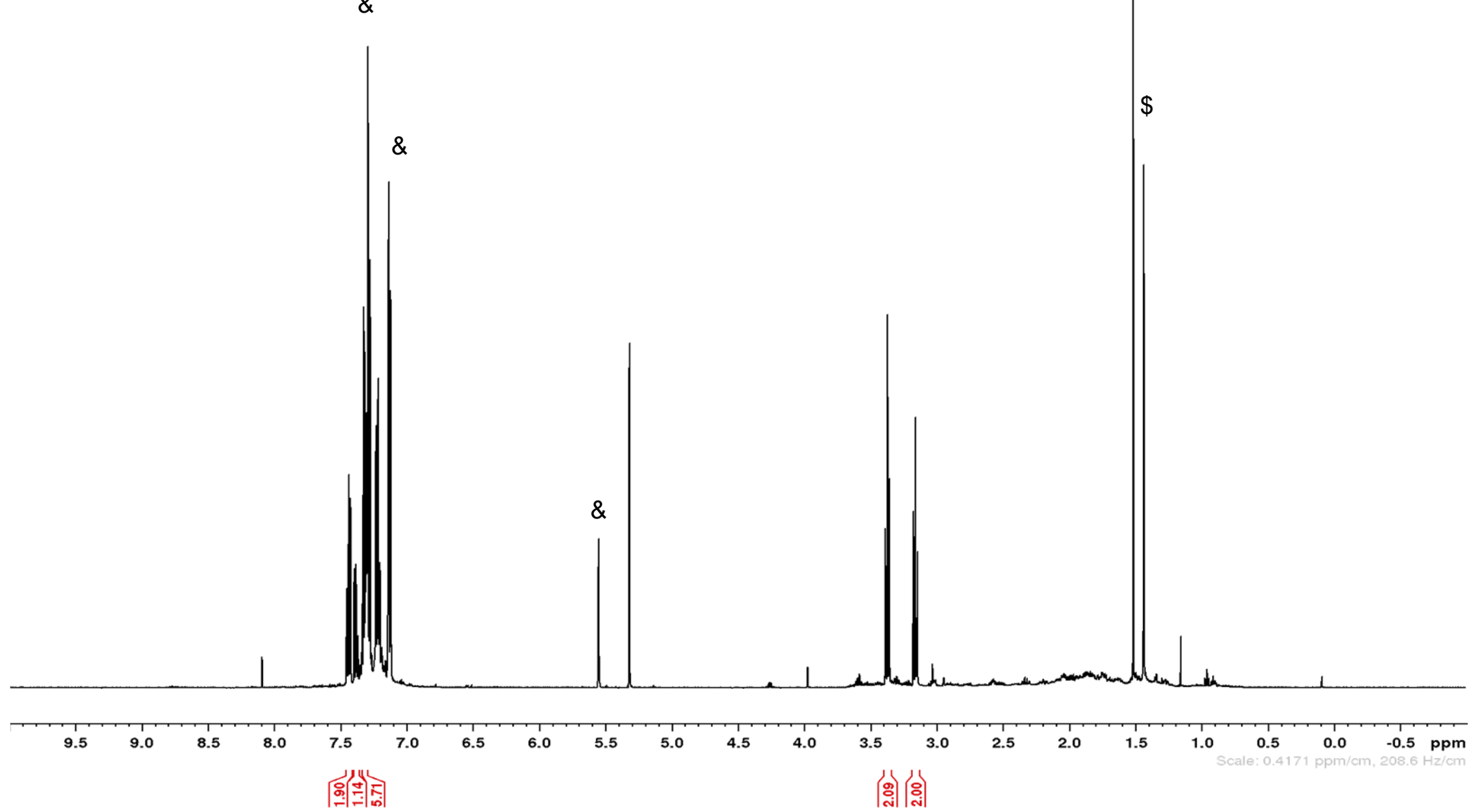


Scheme s74. ${ }^{13} \mathrm{C}\left\{{ }^{1} \mathrm{H}\right\}$ NMR (100 MHz, $\mathrm{CD}_{2} \mathrm{Cl}_{2}, 298 \mathrm{~K}$ ) of 2-(2-bromoethyl)-1,1'-biphenyl (14)
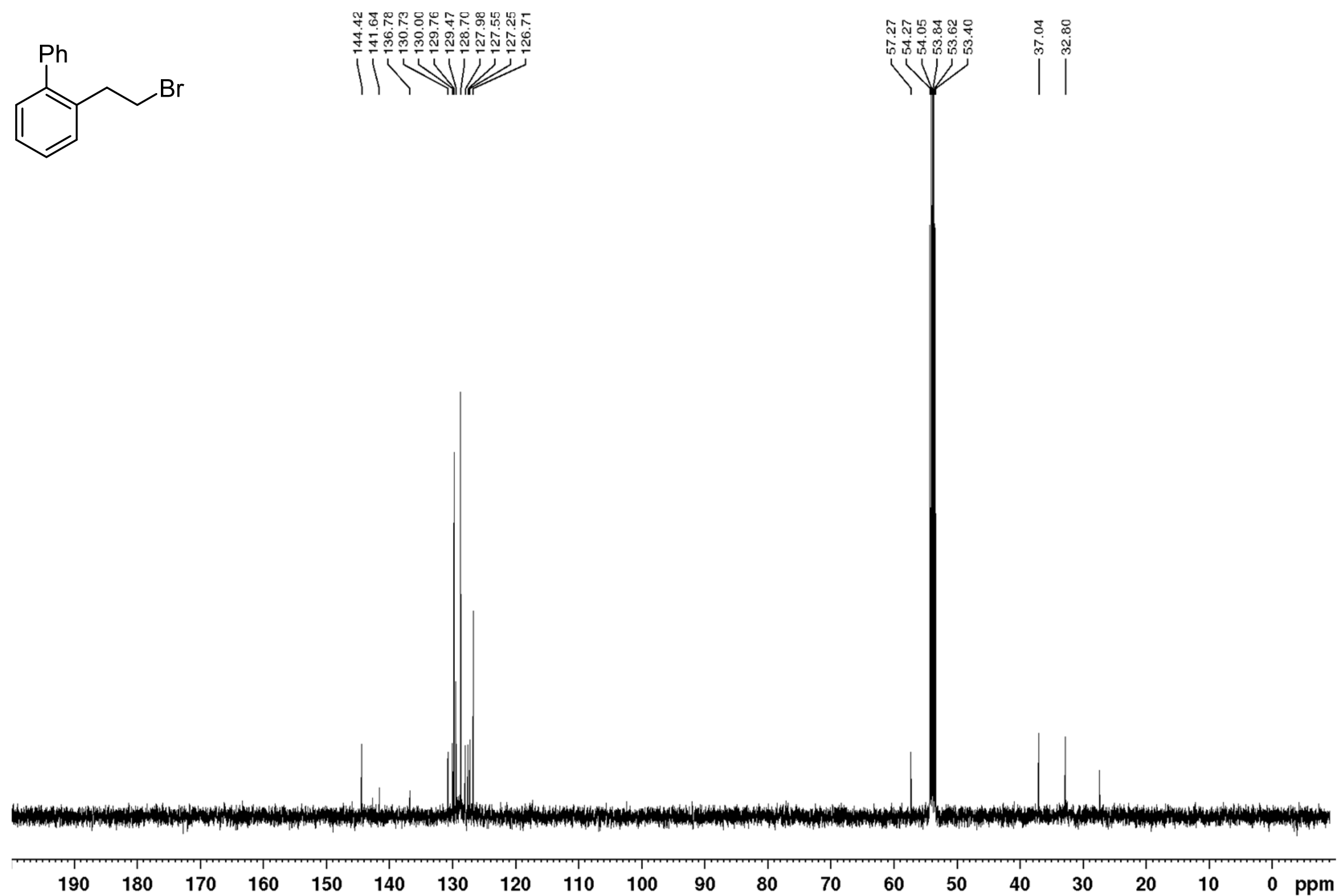
Scheme S75. ${ }^{1} \mathrm{H}$ NMR spectrum (400 MHz, $\left.\mathrm{CDCl}_{3}, 298 \mathrm{~K}\right)$ of 2-ethyl-1,1'-biphenyl (S13)

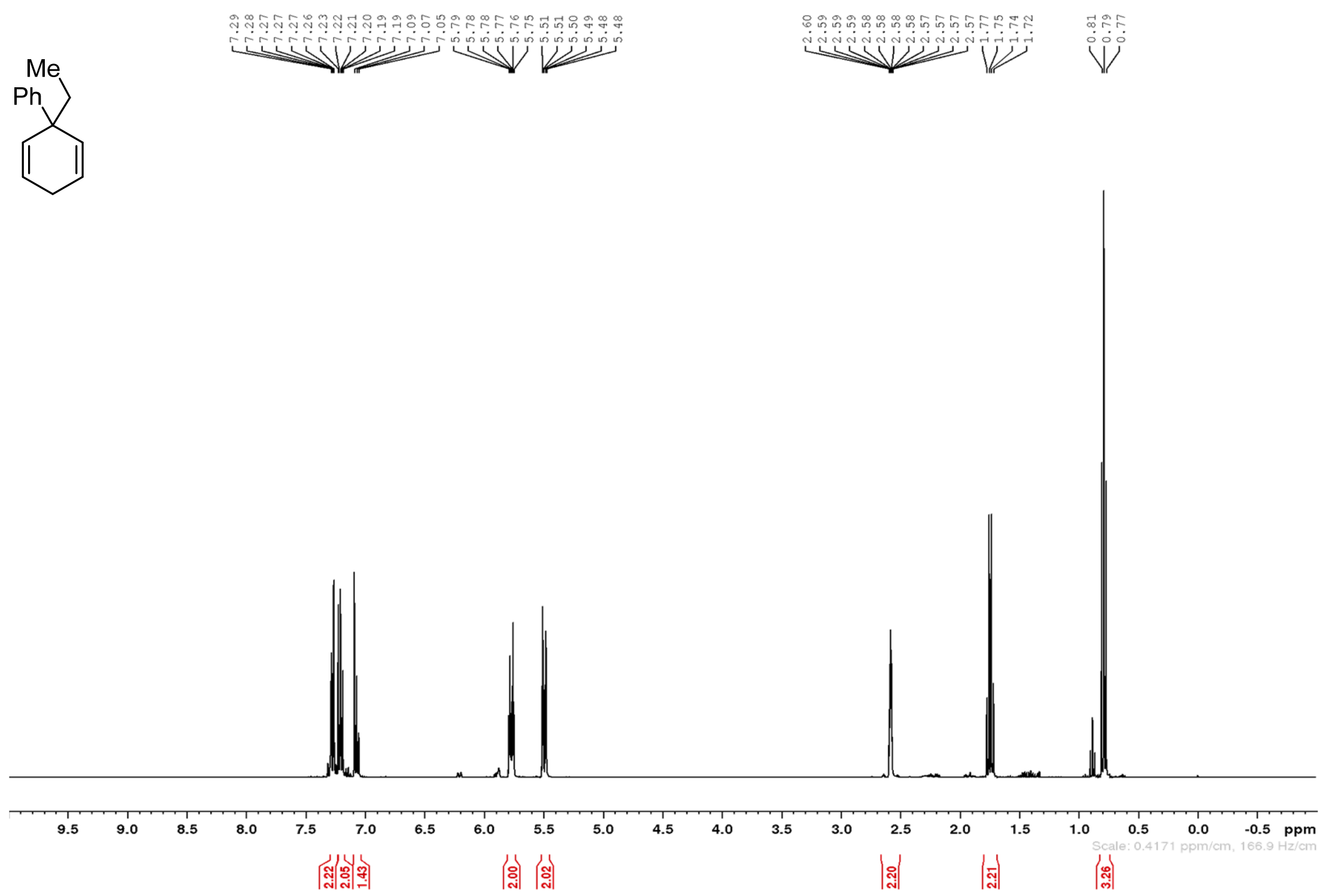


Scheme S76. ${ }^{13} \mathrm{C}\left\{{ }^{1} \mathrm{H}\right\}$ NMR (100 MHz, $\left.\mathrm{CDCl}_{3}, 298 \mathrm{~K}\right)$ of 2-ethyl-1,1'-biphenyl (S13)
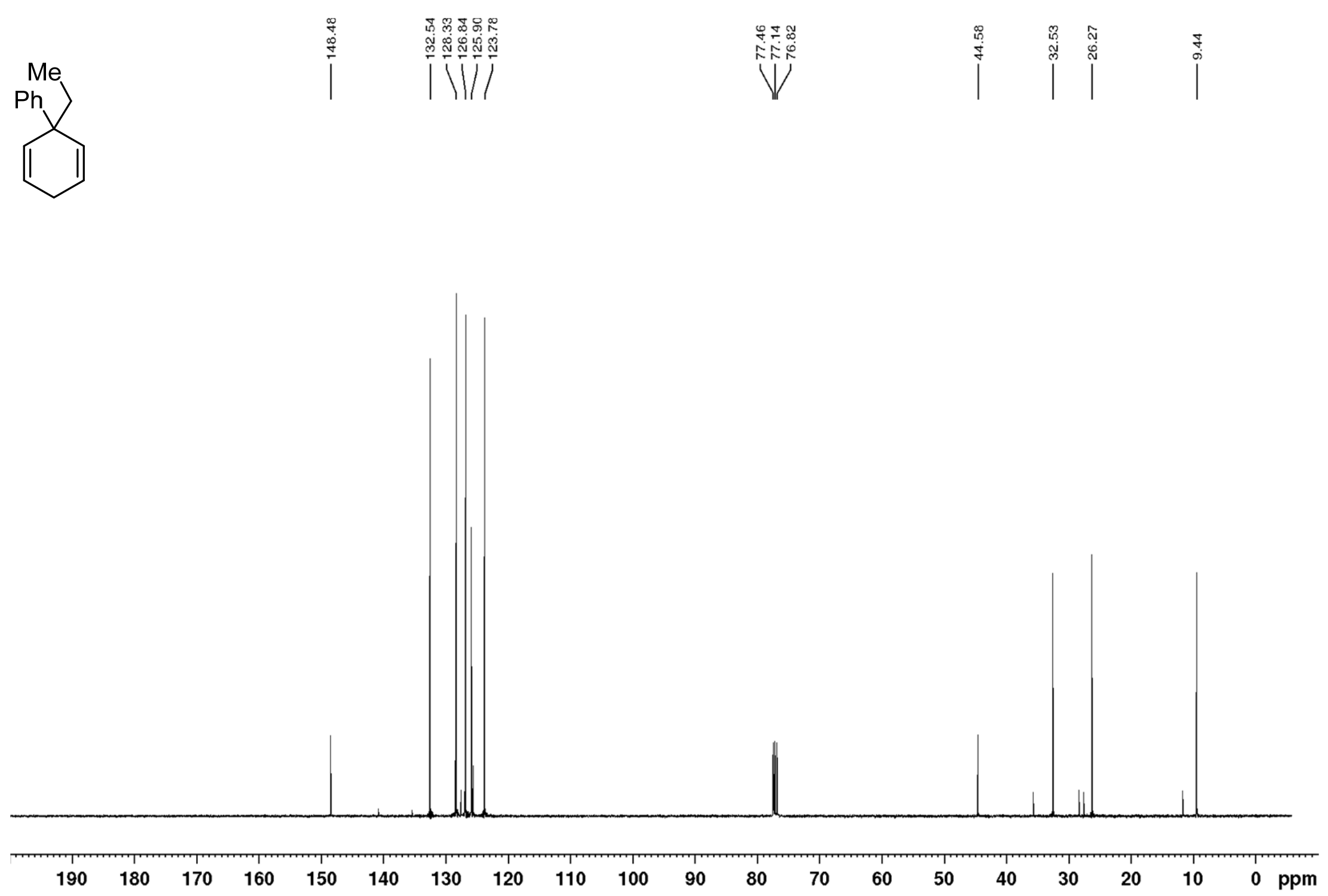
Scheme S77. ${ }^{1} \mathrm{H}$ NMR spectrum (400 MHz, $\left.\mathrm{CD}_{2} \mathrm{Cl}_{2}, 298 \mathrm{~K}\right)$ of 2-ethyl-1,1'-biphenyl (S14) $\left(\#=\mathrm{H}_{2} \mathrm{O}\right)$

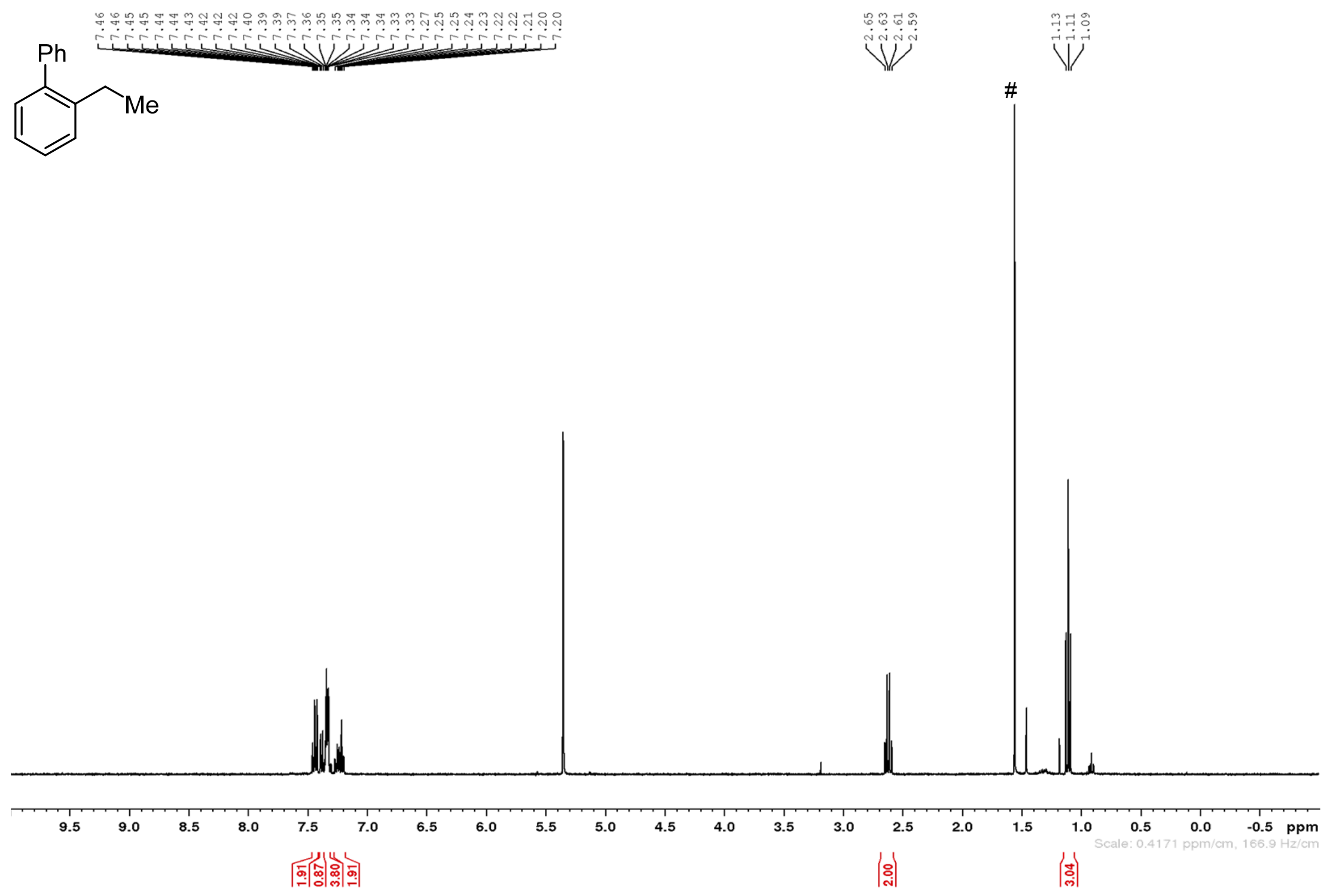


Scheme S78. ${ }^{13} \mathrm{C}\left\{{ }^{1} \mathrm{H}\right\}$ NMR (100 MHz, $\left.\mathrm{CD}_{2} \mathrm{Cl}_{2}, 298 \mathrm{~K}\right)$ of 2-ethyl-1,1'-biphenyl (S14)
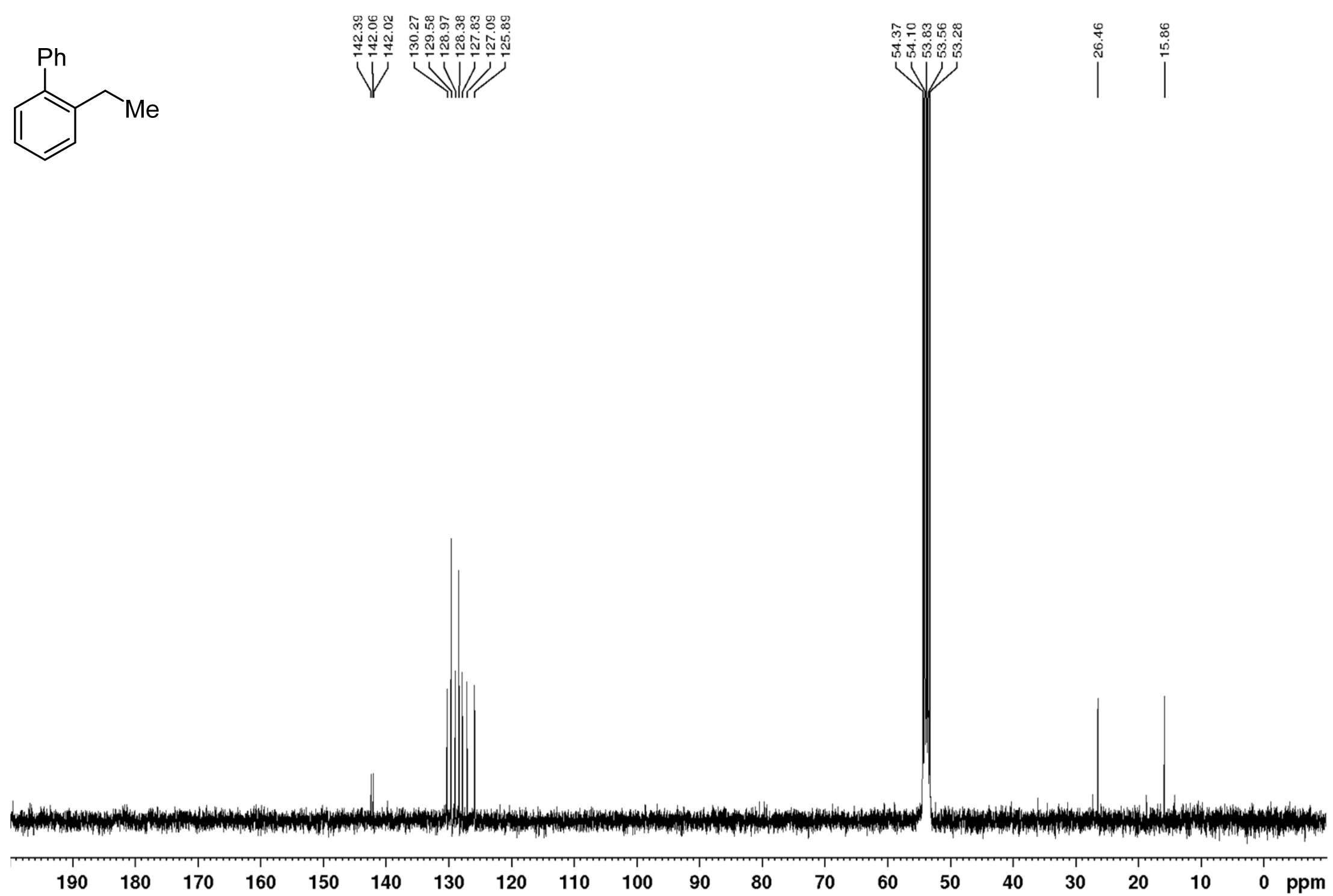


\section{Crystallographic Data}

Data for the single-crystal structure determination were collected with an Agilent SuperNova diffractometer equipped with a CCD area Atlas detector and a mirror monochromator by utilizing $\mathrm{Cu}$-Ka radiation $(\lambda=1.5418 \AA)$. Software packages used: CrysAlis PRO for data collection, cell refinement, and data reduction, ${ }^{\text {S5 }}$ SHELXS-97 for structure solution, ${ }^{\mathrm{S} 6}$ SHELXL-97 for structure refinement, ${ }^{\mathrm{S7}}$ and Mercury ${ }^{\mathrm{S} 8}$ for graphics. 
6.1 Molecular structure of 1-(2-bromodoethyl)-1,4-dihydro-1,1'-biphenyl (3) (CCDC-1907791)

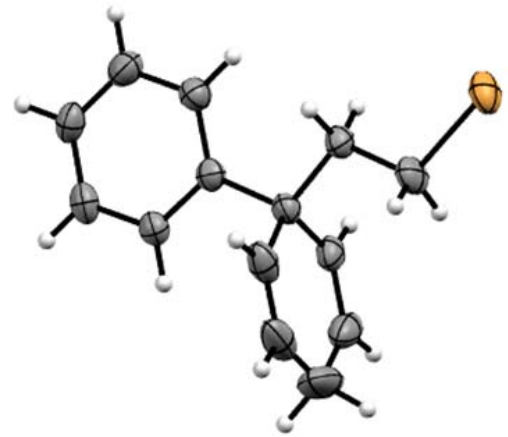

Figure S1: Molecular structure of surrogate 3 (thermal ellipsoids at $50 \%$ probability level; $\mathrm{H}$ atoms except for the water protons omitted for clarity)

Empirical formula

Formula weight

Temperature

Wavelength

Crystal system

Space group

Unit cell dimensions

Volume

Z

Density (calculated)

Absorption coefficient

$\mathrm{F}(000)$

Crystal size

Theta range for data collection

Index ranges

Reflections collected
C14 $\mathrm{H} 15 \mathrm{Br}$

263.17

$150.00(10) \mathrm{K}$

$1.54184 \AA$

Monoclinic

$\mathrm{P} 21 / \mathrm{C}$

$$
\begin{array}{ll}
a=13.6654(5) \AA & a=90^{\circ} . \\
b=7.3494(4) \AA & b=91.611(4)^{\circ} . \\
c=12.1271(5) \AA & g=90^{\circ} .
\end{array}
$$

4

$1.436 \mathrm{Mg} / \mathrm{m}^{3}$

$4.294 \mathrm{~mm}^{-1}$

536

$0.32 \times 0.26 \times 0.09 \mathrm{~mm}^{3}$

3.24 to $67.48^{\circ}$.

$-16<=\mathrm{h}<=16,-8<=\mathrm{k}<=7,-13<=\mathrm{l}<=14$

5596 
Independent reflections

Completeness to theta $=67.48^{\circ}$

Absorption correction

Max. and min. transmission

Refinement method

Data / restraints / parameters

Goodness-of-fit on F2

Final $R$ indices [l>2sigma(I)]

$R$ indices (all data)

Largest diff. peak and hole
$2188[R($ int $)=0.0366]$

$100.0 \%$

Semi-empirical from equivalents

0.6884 and 0.3403

Full-matrix least-squares on $\mathrm{F}^{2}$

2188 / 0 / 136

1.046

$\mathrm{R} 1=0.0452, \mathrm{wR} 2=0.1223$

$\mathrm{R} 1=0.0507, \mathrm{wR} 2=0.1307$

0.867 and -0.926 e. $\AA^{-3}$ 


\section{$7 \quad$ References}

S1 Usui, I.; Nomura, K.; Breit, B. Diastereoselective Hydroformylation of 2,5Cyclohexadienyl-1-carbinols with Catalytic Amounts of a Reversibly Bound Directing Group. Org. Lett. 2011, 13, 612-615.

S2 Levaschov, A. S.; Buryi, D. S. Lewis acid promoted reaction of tetraalkynylstannanes with acyl chlorides: An effective approach towards alkynyl ketones. Tetrahedron Lett. 2017, 58, 4476-4478.

S3 Chen, W.; Walker, J. C. L.; Oestreich, M. Metal-Free Transfer Hydroiodination of C-C Multiple Bonds. J. Am. Chem. Soc. 2019, 141, 1135-1140.

S4 (a) Nogi, K.; Fujihara, T.; Terao, J.; Tsuji, Y. Cobalt-catalyzed carboxylation of propargyl acetates with carbon dioxide. Chem. Comm. 2014, 50, 1305213055. (b) Ashworth, P. J.; Whitham, G. H.; Whiting, M. C. Researches on acetylenic compounds. Part LVIII. The structure of an anomalous Reppe carboxylation product. J. Chem. Soc. 1957, 4633-4640.

S5 Agilent CrysAlis PRO, 2012, Agilent Technologies, Yarnton, UK.

S6 Sheldrick, G. M. Phase annealing in SHELX-90: direct methods for larger structures. Acta Crystallogr., Sect. A. 1990, 46, 467-473.

S7 Sheldrick, G. M. A short history of SHELX. Acta Crystallogr., Sect. A. 2008, 64, 112-122.

S8 Mercury 3.9; Cambrige Crystallographic Data Center:

https://www.ccdc.cam.ac.uk/solutions/csd-system/components/mercury/ 